\title{
THE EFFECTS OF LIGHTNING AND HIGH ALTITUDE ELECTROMAGNETIC PULSE ON POWER DISTRIBUTION LINES
}

\author{
M. A. Uman \\ M. Rubinstein \\ Z. Yacoub \\ University of Florida
}

January 1995

Prepared by

University of Florida

Gainesville, FL 32611

under

subcontract 19X-89650C

for the

Power Systems Technology Program

Energy Division

Oak Ridge National Laboratory

Oak Ridge, Tennessee 37831 managed by

Martin Marietta Energy Systems, Inc.

for the U.S. Department of Energy

under Contract DE-AC05-84OR21400 



\section{DISCLAIMER}

This report was prepared as an account of work sponsored by an agency of the United States Government. Neither the United States Government nor any agency thereof, nor any of their employees, make any warranty, express or implied, or assumes any legal liability or responsibility for the accuracy, completeness, or usefulness of any information, apparatus, product, or process disclosed, or represents that its use would not infringe privately owned rights. Reference herein to any specific commercial product, process, or service by trade name, trademark, manufacturer, or otherwise does not necessarily constitute or imply its endorsement, recommendation, or favoring by the United States Government or any agency thereof. The views and opinions of authors expressed herein do not necessarily state or reflect those of the United States Government or any agency thereof. 


\section{DISCLAIMER}

Portions of this document may be illegible in electronic image products. Images are produced from the best available original document. 
List of Figures . . . . . . . . . . . . . . . . . . . . . v

List of Tables . . . . . . . . . . . . . . . . . . . . . xi

Abstract . . . . . . . . . . . . . . . . . . . . xii

Executive Summary . . . . . . . . . . . . . . . . . . . . xiv

Chapter I - INTRODUCTION AND BACKGROUND . . . . . . . . . . . . . . 1

Chapter II - CATEGORIZATION AND ANALYSIS OF OVERHEAD CLOUD • • • • 5 LIGHTNING DATA

2.1 Categorization of Data . . . . . . . . . . . . . 5

2.2 Location of Cloud Flashes . . . . . . . . . . . . . . 6

2.2 .1 Radar . . . . . . . . . . . . . . . . 7

2.2.2 Three-Station Delay-Time Method ........ . 7

CHAPTER III - CHARACTERIZATION OF LIGHTNING INDUCED VOLTAGES - • 19 AND INDUCING FIELDS FROM VERY CLOSE ARTIFICIALLY INITIATED LIGHTNING

3.1 Introduction . . . . . . . . . . . . . . . . . . 19

3.2 Vertical Electric Fields . . . . . . . . . . . . . . 19

3.3 Induced voltages ................... . 35

3.3 .1 Oscillatory voltages . . . . . . . . . . . . 40

3.3.2 Impulsive Voltages . . . . . . . . . . . . . . 42

3.4 Recent Measurements of Vertical and Horizontal Electric 43 Fields - 30 Meters From Triggered Lightning

CHAPTER IV - CALCULATION OF THE CAUSATIVE FIELDS FOR THE • • • • 47 COUPLING OF CLOUD LIGHTNING FIELDS TO OVERHEAD POWER IINES

4.1 Introduction . . . . . . . . . . . . . . . . . 47

4.2 Incident Plane Wave With Arbitrary Polarization . . . . 47 4.2.1 Introduction ................... 47 4.2.2 Parallel Polarization ............. 48 4.2.3 Perpendicular Polarization . . . . . . . . . 53

4.3 Wavetilt for Non-Grazing Incidence . . . . . . . . . . 54 4.3 .1 Introduction .................. 54 4.3.2 Derivation of Wavetilt Expressions....... 57

CHAPTER V - COUPLING THEORY: TRANSMISSION IINE EQUATIONS • • • . 61 IN THE TIME DOMAIN 
5.1 Frequency-Independent Ground Resistance . . . . . . . . 78 Approximation

5.2 Exact Approach for Including the Finite . . . . . . 80 Conductivity of the Ground

CHAPTER VI - FIELD CALCULATIONS FOR THE COUPLING OF VERY • • - . 83 CLOSE FIELDS TO OVERHEAD POWER IINES

6.1 The Vertical Field Over an Imperfectly . . . . . . . . 83 Conducting Ground

6.2 The Horizontal Field Over an Imperfectly . . . . . . . 84 Conducting Ground

6.2.1 Use of the Wavetilt for the Calculation . . . . 84 of Distant Horizontal Electric Fields

6.2.2 The Calculation of Close Horizontal . . . . . 88 Electric Fields

CHAPTER VII - COMPARISON OF THEORY AND EXPERIMENTAL RESULTS • • • 97 FOR VERY CLOSE LIGHTNING

7.1 Introduction . . . . . . . . . . . . . . . . . 97

7.2 Method of Analysis . . . . . . . . . . . . . . 98

7.3 Discussion ...................... 103

CHAPTER VIII - OVERHEAD CLOUD LIGHTNING: THEORY VERSUS • • • • 127 EXPERIMENT

8.1 Coupling Theory for Overhead Lightning . . . . . . . . 127

8.1.1 Frequency-Domain Coupling Equations . . . . . . 127

8.1.2 EMPLIN ... . . . . . . . . . . ..... 128

8.1.3 An Overview of Coupling ............. 131

8.2 Analysis . . . . . . . . . . . . . . . . . . . . 134

8.3 Results . . . . . . . . . . . . . . . . . . . 137

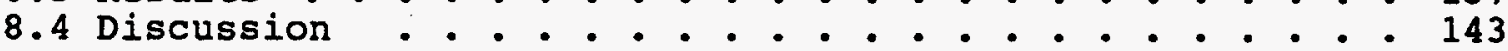

REFERENCES . . . . . . . . . . . . . . . . . . . . . . . 147 


\section{IIST OF FIGURES}

Figure

page

2.1 Output from Daytona Beach National Radar Service at 22:34:00 UT on August 17 (day $\$ 229$ ), 1986. The full range of the radar picture is 250 nautical miles. The Atmospheric science Field Laboratory (ASFL) is located 52.729 kilometers south and 31.396 kilometers east of Daytona Beach which is the center of the radar scan. Notice that there is always a spot at the center of the picture due to the buildings of the Daytona Beach area that can be confused with thunder clouds.

2.2 Output from Daytona Beach National Radar Service at 16:36:00 UT on August 20 (day \#232), 1986. The full range of the radar picture is 250 nautical miles. The Atmospheric Science Field Laboratory (ASFL) is located 52.729 kilometers south and 31.396 kilometers east of Daytona Beach which is the center of the radar scan. Notice that the ASFL is experiencing lightning activity directly above.

2.3 Geometry used in the derivation of the formulae for the three station delay-time method. Note, that $\Phi=0^{\circ}$ in the figure.

2.4 East pole time delay as a function of azimuth and elevation. Only three values of elevation angles are used: $\Phi=0^{\circ}, 42.5^{\circ}$, and $85^{\circ}$.

2.5 West pole time delay as a function of azimuth and elevation. Only three values of elevation angles are used: $\Phi=0^{\circ}, 42.5^{\circ}$, and $85^{\circ}$.

2.6 Measured north-south component of the magnetic field forfive overhead lightning events.

Vertical electric field intensity associated with flash 1 measured $500 \mathrm{~m}$ away from artificially initiated lightning on day 232 at Kennedy Space Center, Florida in 1986. The system decay time is 5 msec.

3.2 Vertical electric field intensity associated with flash 1 measured $500 \mathrm{~m}$ away from artificially initiated lightning on day 240 at Kennedy space Center, Florida in 1986. The system decay time is 5 msec. The pulses preceding the first stroke and the one after the second stroke have rise times of the order of tens of microseconds and hence are not return strokes. 
3.3 Vertical electric field intensity associated with flash 2 measured $500 \mathrm{~m}$ away from artificially initiated lightning on day 240 at Kennedy Space Center, Florida in 1986. The system decay time is $5 \mathrm{msec}$.

3.4 A sketch of the vertical electric field intensity for a typical return stroke showing definitions of some salient parameters.

3.5 Return stroke vertical electric field waveforms measured $500 \mathrm{~m}$ away from artificially initiated lightning and voltages at either end of the test overhead line, one end located $20 \mathrm{~m}$ from the discharge, for lightning flash 1 on day 232 at Kennedy Space Center, Florida in 1986. (a) through (g) represent the seven individual strokes recorded for that flash (see Figure 3.1). The terms oscillatory voltage and impulsive voltage are discussed in the text.

3.6 Return stroke vertical electric field waveforms measured $500 \mathrm{~m}$ away from artificially initiated lightning and voltages at either end of the test overhead line, one end located $20 \mathrm{~m}$ from the discharge, for lightning flash 1 on day 240 at Kennedy Space Center, Florida in 1986. (a) and (b) represent the two individual strokes recorded for that flash (see Figure 3.2). The terms oscillatory voltage and impulsive voltage are discussed in the text.

3.7 Return stroke vertical electric field waveforms measured $500 \mathrm{~m}$ away from artificially initiated lightning and voltages at either end of the test overhead line, one end located $20 \mathrm{~m}$ from the discharge, for lightning flash 2 on day 240 at Kennedy Space Center, Florida in 1986. (a) and (b) represent the two individual strokes recorded for that flash (see Figure 3.3). The terms oscillatory voltage and impulsive voltage are discussed in the text.

3.8 Sketches of typical waveforms. (a) Impulsive voltages. (b) Oscillatory voltages.

3.9 Vertical electric field waveform measured $30 \mathrm{~m}$ from triggered lightning.

3.10 A horizontal electric field waveform measured 30 $\mathrm{m}$ from triggered lightning at a height of $1.5 \mathrm{~m}$ above salt water.

4.1 Definition of the three direction angles 
vii

4.2 The vertical and horizontal components of the parallel-polarized incident electric field.

4.3 Variation of the magnitude of both the paralleland perpendicular-polarized horizontal electric field components with height, elevation angle, and frequency.

4.4 Variation of the magnitude of vertical component of the electric field with height, elevation angle, and frequency.

5.1 Definition of the integration path in the derivation of the first Telegrapher's equation. The ground plane coincides with the $x-y$ plane and the overhead line is in the $x-z$ plane at $z=h$.

5.2 Geometry for the calculation of the magnetic field from a current flowing through an overhead wire for the case of a perfectly conducting ground plane.

5.3 Geometry for the approximate calculation of the magnetic field from a current flowing through an overhead wire for the case of an imperfectly conducting ground plane.

5.4 Plots of the ratio of $\delta \partial \mathrm{H}_{z}(y, 0) / \partial y$ to $\mathrm{H}_{y}(z=0, y)$ as a function of $y$.

5.5 A plot of the percent error between the inductance calculated for a perfectly conducting ground and the inductance calculated for an imperfect ground of $\sigma=1.6 \times 10^{-2}$.

6.1 Definition of the geometry used in the derivation of the wavetilt formula.

6.2 Definition of the geometry used in the calculation of the horizontal electric field over an imperfectly conducting ground.

6.3 Horizontal field calculated over an imperfectly conducting ground for distances of $20 \mathrm{~m}$ and $450 \mathrm{~m}$. In (a) the field is plotted in a $3 \mu \mathrm{sec}$ scale and in (b) in a $25 \mu s e c$ scale. The field has been separated into two components in accordance with Eq. (3.77). The front time constants of the core and corona currents at ground are $0.07 \mu \mathrm{sec}$ and 2.0 usec, respectively. The decay time constants of the core and corona currents at ground are $2.2 \mu \mathrm{sec}$ and $20.0 \mathrm{\mu sec}$, respectively. The amplitudes of the core and corona currents at ground are $5.25 \mathrm{kA}$ and 3.75 $k A$, respectively. The core and corona discharge time constants, 0.1 Hsec and 6.0 Hsec, respectively. The striking height, $20 \mathrm{~m}$. 


\section{viii}

7.1 A channel-base current used in the analysis showing the separation into currents $A$ (core current) and current B (corona current).

7.2 Channel-base current (upper left), calculated oscillatory voltages at the east (lower right) and west (lower left) ends of the line, and vertical electric field intensity (upper right) calculated to simulate the second stroke of flash 1 that occurred on day 232. Experimental data are found in Figure $3.5 \mathrm{~b}$.

7.3 Channel-base current (upper left), calculated oscillatory voltages at the east (lower right) and west (lower left) ends of the line, and vertical electric field intensity (upper right) calculated to simulate the sixth stroke of flash 1 that occurred on day 232. Experimental data are found in Figure 3.5f.

7.4 Channel-base current (upper left), calculated oscillatory voltages at the east (lower right) and west (lower left) ends of the line, and vertical electric field intensity (upper right) calculated to simulate the first stroke of flash 1 that occurred on day 240. Experimental data are found in Figure 3.6a.

7.5 Channel-base current (upper left), calculated oscillatory voltages at the east (lower right) and west (lower left) ends of the line, and vertical electric field intensity (upper right) calculated to simulate the second stroke of flash 1 that occurred on day 240. Experimental data are found in Figure $3.6 \mathrm{~b}$.

7.6 Channel-base current (upper left), calculated oscillatory voltages at the east (lower right) and west (lower left) ends of the line, and vertical electric field intensity (upper right) calculated to simulate the first stroke of flash 2 that occurred on day 240. Experimental data are found in Figure 3.7a.

7.7 Channel-base current (upper left), calculated oscillatory voltages at the east (lower right) and west (lower left) ends of the line, and vertical electric field intensity (upper right) calculated to simulate the second stroke of flash 2 that occurred on day 240 . Experimental data are found in Figure $3.7 \mathrm{~b}$.

7.8 Simulation of corona effects. The capacitance matrix of the line was changed to account for the increase in the radius of the wires due to corona and the resistance per unit length was set to 1 
$\mathrm{Ohm} / \mathrm{m}$ to model the losses due to corona. The time between successive reflected pulses is $3.1 \mu \mathrm{\mu ec}$. Experimental data are shown in Figure $3.5 \mathrm{~b}$.

Simulation of corona effects. The capacitance matrix of the line was changed to account for the increase in the radius of the wires due to corona and the resistance per unit length was set to 1 $\mathrm{ohm} / \mathrm{m}$ to model the losses due to corona. The time between successive reflected pulses is $3.1 \mu \mathrm{sec}$. Experimental data are shown in Figure $3.5 f$.

7.10 Simulation of corona effects. The capacitance matrix of the line was changed to account for the increase in the radius of the wires due to corona and the resistance per unit length was set to 1 $\mathrm{Ohm} / \mathrm{m}$ to model the losses due to corona. The time between successive reflected pulses is $3.1 \mu s e c$. Experimental data are shown in Figure 3.6a.

7.11 Simulation of corona effects. The capacitance matrix of the line was changed to account for the increase in the radius of the wires due to corona and the resistance per unit length was set to 1 $\mathrm{Ohm} / \mathrm{m}$ to model the losses due to corona. The time between successive reflected pulses is $3.1 \mu \mathrm{sec}$. Experimental data are shown in Figure $3.6 \mathrm{~b}$.

7.12 Simulation of corona effects. The capacitance matrix of the line was changed to account for the increase in the radius of the wires due to corona and the resistance per unit length was set to 1 $\mathrm{Ohm} / \mathrm{m}$ to model the losses due to corona. The time between successive reflected pulses is 3.1 Hsec. Experimental data are shown in Figure $3.7 a$.

7.13 Simulation of corona effects. The capacitance matrix of the line was changed to account for the increase in the radius of the wires due to corona and the resistance per unit length was set to 1 $\mathrm{Ohm} / \mathrm{m}$ to model the losses due to corona. The time between successive reflected pulses is 3.1 Hsec. Experimental data are shown in Figure $3.7 \mathrm{~b}$.

7.14 A photograph showing the physical arrangement of the resistive terminations and the voltage dividers. Points $A$ and $B$ are the top screw terminals, and points $C$ and $D$ are the center clamps.

7.15 Current used for simulation of the electric field intensity and voltages produced by the fifth stroke in flash 1 occurred on day 232. 
7.16 Vertical electric field intensity at $500 \mathrm{~m}$ and voltages at either end of the test line calculated by simulating flashovers (defined in the text) at both ends of the line. The flashover voltages at each end were chosen to obtain amplitudes consistent with those in the fifth stroke in flash 1 occurred on day 232 (see Figure 3.5e).

8.1 Definition of polarization quadrants.

8.2 Calculated incident electric field for five events.

8.3 Effect of the ground conductivity on the simulated voltages for event 19 of flash 86. From top to bottom, the pairs of voltages were calculated with $\sigma=1.6 \times 10^{-2}, 1.0 \times 10^{-2}, 0.5 \times 10^{-2}$, and 0.25 x $10^{-2} \mathrm{mho} / \mathrm{m}$.

8.4 Illustration of the effect of lowering the elevation angle in the simulation of event 19 of flash 86 


\section{IIST OF TABLES}

Table

3.1

3.2

3.3

3.4

3.5

8.1

8.2

8.3

Amplitudes and risetimes of electric field $R$

Page changes. All the parameters are defined in Figure 3.4 .

parameters of oscillatory voltages at the east end of the test line. All the parameters are defined in Figure $3.8 \mathrm{a}$.

Some parameters of oscillatory voltages at the west end of the test line. All the parameters are defined in Figure $3.8 \mathrm{a}$.

Some parameters of impulsive voltages at the west end of the test line. All the parameters are defined in Figure $3.8 \mathrm{~b}$.

Some parameters of impulsive voltages at the east end of the test line. All the parameters are defined in Figure $3.8 \mathrm{~b}$.

The addition characteristic of the four polarization quadrants with respect to the four regions of the azimuth plane.

The variation in $A$ as a function of azimuth for $\alpha=80^{\circ}$ and $\Phi=80^{\circ}$.

The convention used by EMPLIN to determine in which quadrant the incident electric field lies. 
xii

\section{ABSTRACT}

During the summer of 1986 , an experiment was conducted by the University of Florida Lightning Research Group at the National Aeronautics and Space Administration (NASA) Kennedy Space Center (KSC). The group simultaneously recorded the voltages induced by lightning on both ends of an unenergized 448-meter long unenergized electric power line and the lightning vertical electric and horizontal magnetic fields at ground level near the line. The lightning data studied and presented here were due both to cloud lightning and to very close (about $20 \mathrm{~m}$ from the line) artificially initiated lightning. For cloud sources, a frequency-domain computer program called EMPLIN was used to calculate induced line voltages as a function of source elevation, angle of incidence, and wave polarization of the radiated cloud discharge pulses in order to compare with the measurements. For very-close lightning, the measured line voltages could be grouped into two categories, those in which multiple, similarly shaped, evenly spaced pulses were observed, which we call oscillatory, and those dominated by a principal pulse with subsidiary oscillations of much smaller amplitude, which we call impulsive. The amplitude of the induced voltage ranged from tens of kilovolts for oscillatory voltages to hundreds of kilovolts for impulsive voltages. A new technique is derived for the calculation of the electromagnetic fields from nearby lightning to ground above an imperfectly conducting ground. This technique was used in conjunction with an existing time domain coupling theory and lightning return stroke model to calculate voltages at either end of the line. The results show fair agreement with the measured oscillatory voltage waveforms if corona is ignored and improved results when corona effects are modeled. The modeling of the impulsive voltage, for which local flashover probably 
xiii

occurred, were not successful. In an attempt to understand better the sources of the line voltages for very close lightning, measurements of the horizontal and vertical electric fields $30 \mathrm{~m}$ from triggered lightning were obtained during the summer of 1991. 
xiv

\section{EXECUTIVE SUMMARY}

Measured electric and magnetic fields and associated measured induced voltages on a $448 \mathrm{~m}$ overhead unenergized electric power distribution line produced by both overhead cloud flashes and close artificially initiated ground flashes are analyzed. Theory is developed to obtain rough locations of cloud discharges and to calculate the causative cloud lightning electric fields given measured magnetic fields. The EMPLIN code is used to determine line voltages with the cloud electric fields as inputs. Measured induced voltages from very close, about $20 \mathrm{~m}$, artificially-initiated lightning to ground and the corresponding vertical electric fields at $500 \mathrm{~m}$ are thoroughly characterized, and theory is developed for the calculation of the causative vertical and horizontal electric fields in the vicinity of the triggered lightning channel. Electric fields $30 \mathrm{~m}$ from those channels are measured. Theoretical induced line voltages are obtained using a time domain coupling theory, and the results are compared to the measured voltages.

Cloud discharge pulses with high elevation angles produce voltages of opposite initial polarities at each end of the line due to the fact that the pulse electric fields are essentially horizonal. Multiple oscillations are observed when both ends of the line are open-circuited. All significant observed voltage features can be reproduced with the frequency domain coupling code EMPLIN.

When the ground lightning return stroke is located very close to the line, measurements indicate the existence of two types of voltages at the ends of the line. These have been termed impulsive and oscillatory. Voltages of the impulsive type exhibit a dominant pulse and a series of subsidiary pulses with peak amplitudes less than one-fifth the peak 
XV

amplitude of the main pulse. Voltages of the oscillatory type, on the other hand, consist of a series of pulses with similar shapes and decaying amplitudes, although the second pulse is generally larger than the first pulse. Peak oscillatory voltages are of the order of $50 \mathrm{kV}$, whereas peak impulsive voltages are an order of magnitude higher. Oscillatory voltages can be modeled fairly well with existing theory. Impulsive voltages cannot be and may be due to electrical breakdown in the measuring system. 


\section{CHAPTER I \\ INTRODUCTION AND BACKGROUND}

Electromagnetic fields interact with conductors by inducing currents in them. In the case of electric power lines, these currents result in line voltages. Iightning, being a natural process that produces propagating electromagnetic waves, is capable of inducing such power line currents. Lightning can be divided into two major types: ground and cloud lightning. Overhead cloud lightning, being an elevated source of electromagnetic radiation, can be used to study the adequacy of high altitude electromagnetic pulse coupling models. All the lightning studies on electric power systems that have been performed prior to 1980 assume that the voltages induced on power lines from lightning are due only to the vertical component of the electric field. The reason behind this assumption is that the ground is a reasonably good conductor at the frequencies involved, so that the horizontal component of the electric field might be expected to be small (compared to the vertical component) and thus not to play an important role in the coupling process. The first experimental work indicating the importance of the horizontal component of the electric field in the coupling process was published by Koga et al. (1981). Further experimental evidence of the significance of the horizontal component of the electric field in producing line voltages has been provided by the Lightning Research Group of the University of Florida (UF) with support from the Department of Energy. The UF group conducted an experiment in the summer of 1979 in the Tampa Bay area in which they simultaneously measured lightning electric fields and induced voltages at one end of the power line. The horizontal electric field component, which was calculated from the measured vertical 
electric field component using the wavetilt formula, was shown to play an important role in the coupling mechanism (Master, 1982; Master et al., 1984; and Master and Uman, 1984). The measured voltage waveshapes were in fairly good agreement with voltages calculated using the time domain model of Agrawal et al. (1980). The amplitudes of the calculated voltages, on the other hand, were a factor of 4 lower than the measured ones. Reasons for this error are discussed in Rubinstein et al. (1989). Primarily, we suspect a calibration error in the measurement system. A more complete experiment, also supported by the Department of Energy, was conducted by the Lightning Research Group in the summer of 1985 at the Kennedy Space Center's Atmospheric Science Field Laboratory (ASFL). The two horizontal components of the lightning magnetic field and the induced voltage at one end of an unenergized power line were simultaneously measured. Once again, the importance of the horizontal electric field in the coupling process was demonstrated. Again, the waveshapes of the measured and calculated voltages were in fairly good agreement.

An additional and expanded experiment was conducted by the University of Florida Lightning Research Group and supported by the Department of Energy at the Kennedy Space Center's ASFL in the summer of 1986. In this experiment, the three electric field components (Thomson et al., 1988a); the two horizontal magnetic field components; and, for the first time, the voltages induced at both ends of an unenergized power line were simultaneously measured. A total of 5500 events were recorded in a period of nine days. A study of the induced ground lightning voltages in the 1986 experiment was presented in a Master's thesis by Georgiadis (1988) and in a journal article by Georgiadis et al. (1992). Georgiadis compares the measured induced voltages at both ends of the power line to the theoretically calculated voltages which were obtained 
by employing a modification of the time domain algorithm used by Master and Uman (1984). The comparison showed very good agreement in the voltage waveshapes and reasonable agreement in the amplitudes. The calculated voltages were larger than the measured, presumably because the calculations were performed using electromagetic fields measured away from the line whereas the fields reaching the line were reduced in amplitude due to shielding by trees along the line and a structure at one end of the line.

The 1986 data also include voltages and fields from both cloud lightning, which have bee analyzed using EMPLIN, a frequency domain coupling code described in Section 8.1.2, and from very close artificially-initiated lightning to ground. In a related study during the summer of 1991, the University of Florida group measured horizontal and vertical electric fields $30 \mathrm{~m}$ from artificially-initiated lightning. In this report, we present the characterization of these additional 1986 and 1991 data, as well as theory developed to model the coupling of overhead and of very close fields to power lines. 


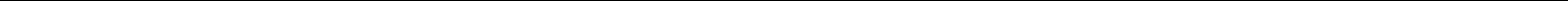




\section{CHAPTER II}

CATEGORIZATION AND ANALYSIS OF OVERHEAD CLOUD IIGHTNING DATA

\subsection{Categorization of Data}

The categorization of the data gathered in 1986 required that all the recorded events be viewed and organized. Each event was organized under three major types: noise, calibration, and data. The "data" events were classified under another two classifications: (i) cloud-to-ground discharge event and (ii) cloud discharge event. All the above classifications were done in Geogiadis' (1988) master's thesis. An event was classified as a component pulse of a cloud discharge if the zerocrossing time of any pulse fields was between 0 and $30 \mu s$ (Krider et al., 1975; Weidman and Krider, 1979; LeVine, 1980). An event was classified as a return stroke in a ground discharge if the zero-crossing time of any waveform was more than $45 \mathrm{\mu s}$ and had the same field characteristics as documented by Lin et al. (1979). An event was called 'questionable' when the waveforms did not fall into either category, the amplitude was too small to be characterized, or the waveform had an atypical shape. With the above classification, 2239 cloud-to-ground discharge events and 385 cloud discharge events were identified.

Each of the cloud discharge events identified by Georgiadis (1988) was examined. Information on whether the cloud pulses were unipolar or bipolar and if the polarity was negative or positive is listed. The type of termination on each end of the power line and the location of lightning activity relative to the KSC Atmospheric science Field Laboratory (ASFL), located about $100 \mathrm{~m}$ from one end of power line, from radar records were documented. Whether the initial pulse peak of the induced voltages at either end of the line had the same or opposite 
polarity was noted. This information is useful in determining the direction of the lightning pulse propagation. Comments were also made if any oscillations were noticed on the two end-voltage records. Further categorization, for cloud discharge events that occurred in the same flash with cloud-to-ground discharge events, was done to help in narrowing the range of possible polarization angles. For example, cloud discharge processes that occurred at the end of the preliminary breakdown process or the beginning of the step leader process in a specific flash were identified. If the cloud discharge event occurred 10-30 msec prior to the return stroke (Kitagawa and Brook, 1960), the cloud discharge is considered as one that occurred at the beginning or during the step leader process which lowers negative charge from the cloud in most cases.

About 608 of all analyzed cloud discharge processes occurred on day 232 of 1986. Only three types of data were recorded on that day: the two end voltages and the north-south component of the magnetic flux density. Most of these cloud discharges have low elevation angles and hence are not a good test for the theory of coupling from elevated sources. There are 227 events, all occurring on day 232, that are due to lightning activity near and above the power line. The events are the ones of primary interest to the present study.

\subsection{Location of Cloud Flashes}

The location of the cloud pulses relative to the power line is defined by two angles: the azimuth angle, $\phi$, and the elevation angle above the horizon, $\Phi$. These angles are needed as input to the EMPLIN computer code in order to calculate the voltages induced at the two ends of the power line. In this section, a time delay method that was used to determine these angles is discussed. Also, radar records that helped 
(only for cloud sources far away from the line) in checking the azimuth angle determined from the above method are presented.

$\underline{2.2 .1 \quad \text { Radar }}$

Radar records on $16 \mathrm{~mm}$ film from Daytona Beach National Radar Service were available for each day of the experiment every 10 to 15 minutes for a full range $\left(360^{\circ}\right.$ ) of 250 nautical miles ( 1 nautical mile -

$1.853 \mathrm{~km}$ ). These records were used to check the validity of the azimuth angle calculated from the time-delay method for sources more than 50 miles away from the power line. For example, if the time-delay method predicts a given azimuth angle, we expect to see radar echoes, in the same general direction. Figures 2.1 and 2.2 show radar pictures for day 229 and day 232, respectively. Day 229 and day 232 were chosen because of the large number of cloud flashes on those days. As can be seen from Figure 2.1 the lightning activity is distributed south and north-west of the ASFL about 100 nautical miles (nm) away. Figure 2.2 shows strong thunderstorm activity directly above the ASFL and to the south of it, about $50 \mathrm{~nm}$ away.

\subsubsection{Three Station Delay-Time Method}

In this section we present the theory of the three-station delaytime method. The delay-time method relies on the difference in the time of arrival of the radiation fields produced by cloud discharge processes at the different measurement sights. For example, with two receivers the azimuth of a radiating source can be obtained as has been done by cianos et al. (1972), Murty and MacClement (1973), and MacClement and Murty (1978). With three receivers one can obtain both azimuth and elevation of the source as was done by Taylor (1978). Lewis et al. (1960) determined the direction of transatlantic lightning with four Very Low Frequency (VLF) stations in New England, each separated by over $100 \mathrm{Km}$. 


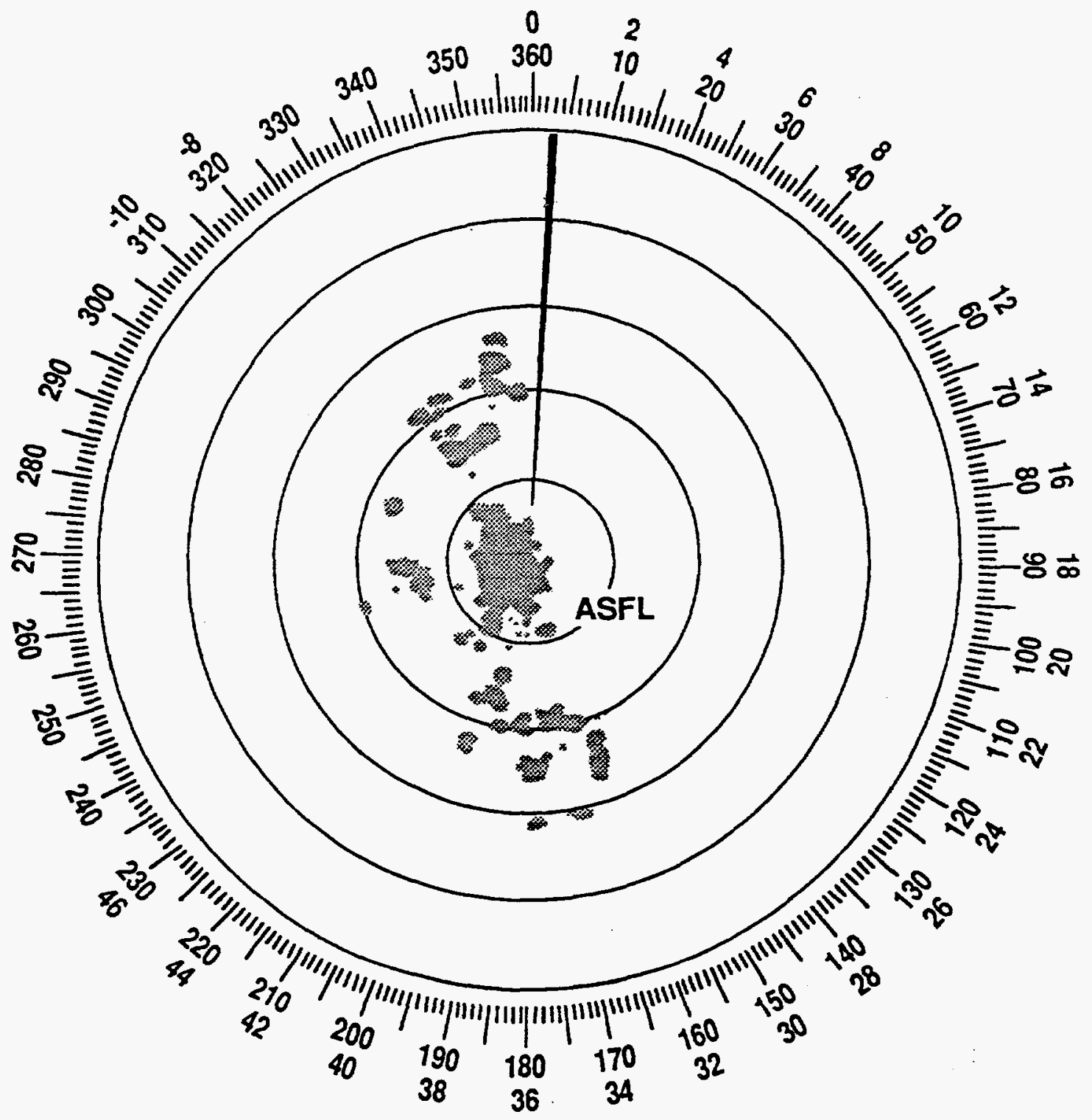

Fig. 2.1 Output from Daytona Beach National Radar Service at 22:34:00 UT on August 17 (day \#229), 1986. The full range of the radar picture is 250 nautical miles. The Atmospheric Science Field Laboratory (ASFL) is located 52.729 kilometers south and 31.396 kilometers east of Daytona Beach which is the center of the radar scan. Notice that there is always a spot at the center of the picture due to the buildings of the Daytona Beach area that can be confused with thunder clouds. 


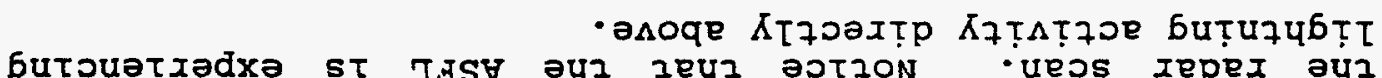

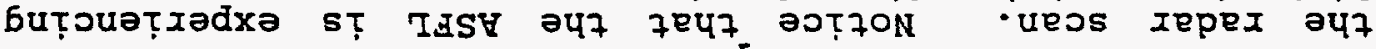

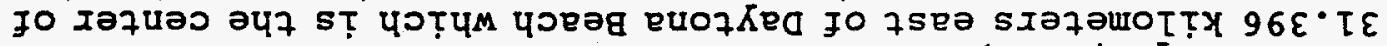

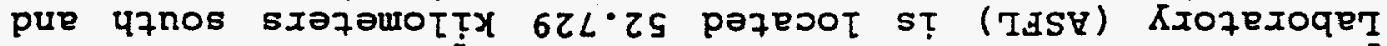

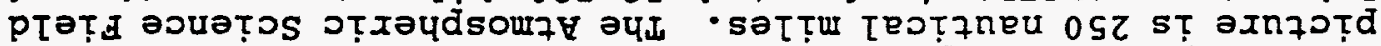

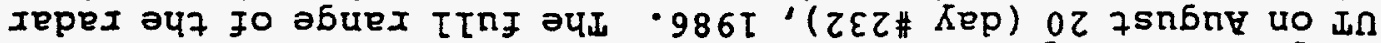

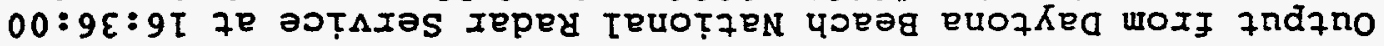

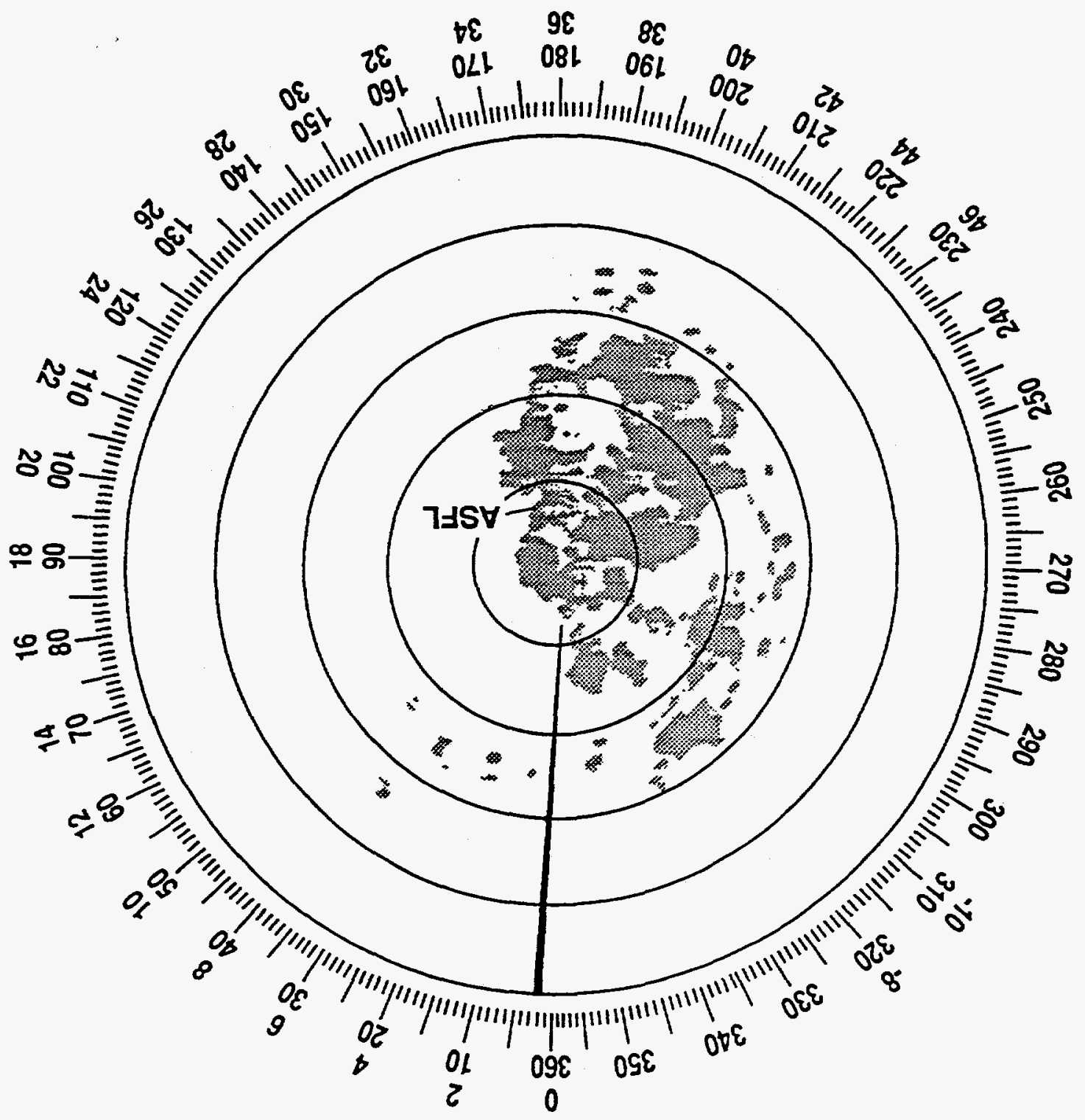


Proctor $(1971,1976,1981 a)$ was able to reconstruct the lightning channel from the individual sources located with five ground receiving stations (centered at $253 \mathrm{MHz}$ with a 5-MHz bandwidth) separated by between 10 and $30 \mathrm{~km}$.

In this experiment we use a three-station receiving system with wide band receivers. The three stations are: (1) a loop antenna ( $3 \mathrm{~Hz}$ to 800 kHz) that measures horizontal magnetic fields at the ASFL building, (2) the voltage at the top phase of the east end of the test distribution line used in the 1988 and 1986 experiments, and (3) the voltage at the west end of the same test line (upper frequency response of the voltage measuring system at least $700 \mathrm{kHz}$ ). Those three measurement stations enabled us to obtain, at least in quadrants, the azimuth and the elevation of the cloud discharge process relative to the power line.

The magnetic loop antenna was mounted on top of the ASFI, a small building where the recording system was located. The coaxial cable running from the loop antenna to the recording channel was only about six meters in length. Knowing that the signal travels down the coaxial cable at about two thirds the speed of light, the time taken by the signal to reach the recording channel was about $30 \mathrm{nsec}$. With the digitizing rate set at $20 \mathrm{MHz}$ or $50 \mathrm{nsec} / \mathrm{sample}$ the $30 \mathrm{~ns}$ delay due to the coaxial cable from the loop antenna to the digital recorder (LeCroy) could not be distinguished by our recording system. Therefore, without introducing any appreciable error, the loop antenna was considered as our reference station, and, thus, the time at which the magnetic signals reached the recording system was considered $t=0$. The length of the fiberoptics cables from the east and west end of the line to the recording system was $700 \mathrm{~m}$ and $500 \mathrm{~m}$, respectively. The difference in the time of arrival of the two end voltages at the recording system due 
to the electronics and fiberoptic cables was measured to be $1.07 \mu \mathrm{sec}$. Since the delay due to the electronics is negligible, the difference can be attributed completely to the difference in the fiberoptic cable length which is $200 \mathrm{~m}$. Thus the speed of the signal down the fiberoptic cable can be easily calculated to be:

$$
\mathrm{V}_{\text {fibex }}=200 / 1.07=1.87 \times 10^{8} \mathrm{~m} / \mathrm{sec} \text {. }
$$

knowing the speed of the signal down the fiberoptic cable, we can calculate the time delay of the measured voltage signal from each end of the line to the recording system. If we call the time delay due to the fiberoptic cable from the west end of the line to the recording system, $t_{\text {wp }}$ and the time delay due to the fiberoptic cable from the east end of the line to the recording system, $t_{\text {op }}$, then:

$$
\begin{aligned}
& t_{\text {wp }}=500 / v_{\text {fiber }}=2.68 \mu \mathrm{sec} \\
& t_{\text {op }}=700 / v_{\text {fiber }}=3.74 \mu \mathrm{sec}
\end{aligned}
$$

Since the digitizing rate is $50 \mathrm{nsec} / \mathrm{sample,} t_{\mathrm{wp}}$ corresponds to advancing the west-end induced voltage record 54 samples, and $t_{\text {op }}$ corresponds to advancing the east-end induced voltage record 75 samples. After that correction, the time differences between the $B_{\mathrm{EW}}, \mathrm{V}_{\mathrm{W}}$, and the $\mathrm{V}_{\mathrm{E}}$ signals were due only to the position of the cloud discharge process relative to the power line.

The next step, after removing all the delays that are inherent in the system, was to derive the mathematical relationships that relate the time delays to the azimuth and elevation angles. Figure 2.3 shows the relative positions of the three measurement stations with the definition of the azimuth angle, $\phi$, and the EM wave front.

According to the previous definition of $t_{\mathrm{vp}}$ and with the help of the geometry in Figure 2.3, we can write an expression for $t_{\text {wp }}$ : $t_{\mathrm{vp}}=\mathrm{OW} / \mathrm{v}_{\mathrm{c}} \quad$ Where $\mathrm{OW}=\mathrm{PW} \cdot \cos \phi$ and $\mathrm{v}_{\mathrm{c}}$ is the speed of light. 


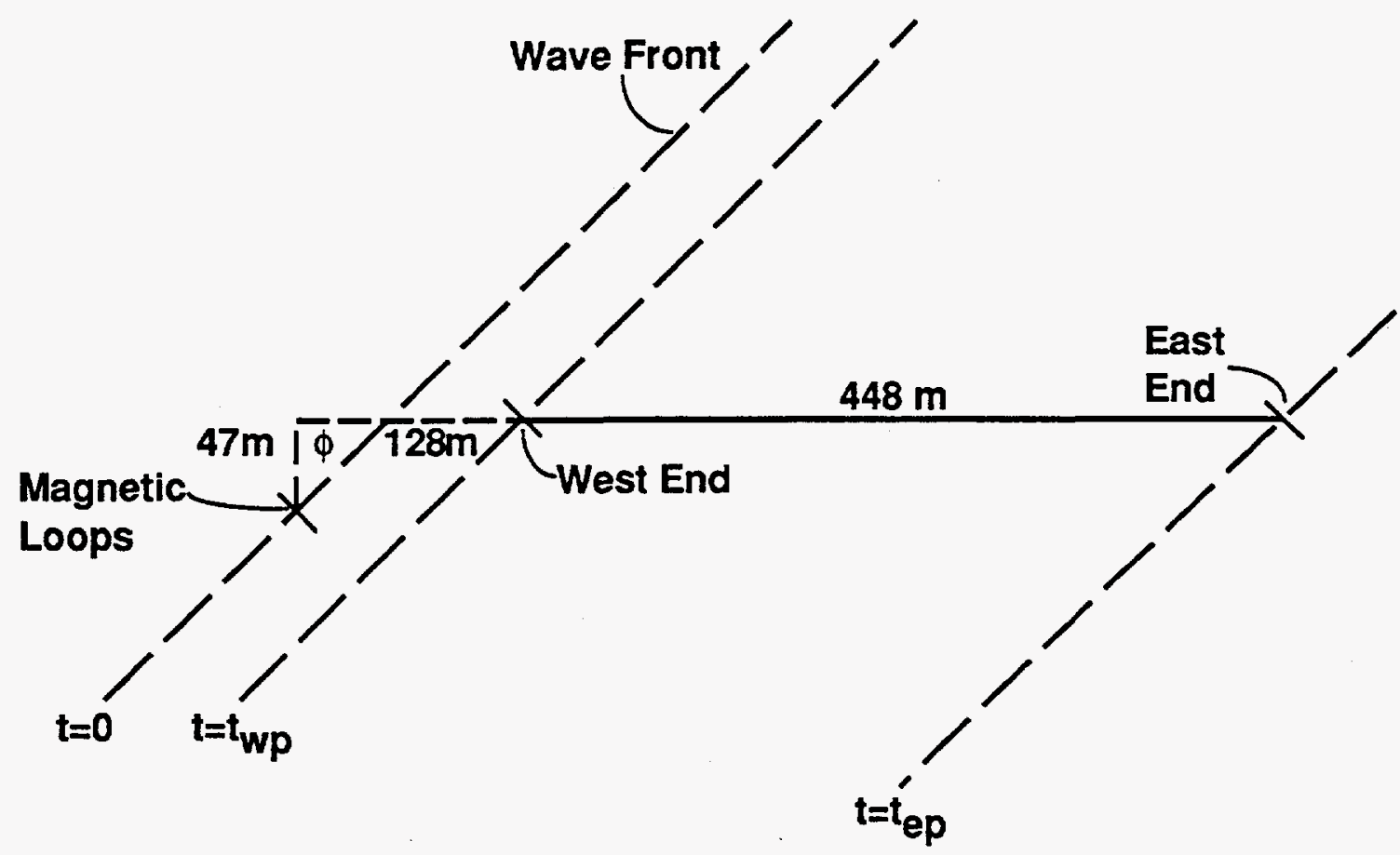

Fig. 2.3 Geometry used in the derivation of the formulae for the three station delay-time method. Note, that $\Phi=0^{\circ}$ in the figure. 
Now, $P W=L W-L P$ where $L P=L M \cdot \tan \phi$. Thus, the expression for $t_{\mathrm{vp}}$ in terms of known distances and $\phi$ can be written as follows

$$
t_{w p}=\frac{L W \cdot \cos \phi-L M \cdot \sin \phi}{V_{C}}
$$

In a similar fashion, we can write an expression for $t_{\text {op }}$ in terms of known distances and $\phi$

$$
t_{e p}=\frac{L E \cdot \cos \phi-L M \cdot \sin \phi}{V_{c}}
$$

Notice that in Figure 2.3 the elevation angle of the source, $\Phi$, was not considered. However, the only change in the derivation of Eq. (2.1) and (2.2) will be the change of $O W$ and $O Q$ to $O W \cdot \cos \Phi$ and $O Q \cdot \cos \Phi$, respectively, since the effect of an elevated source on the magnitude of the time delay is to decrease it by the factor cos $\Phi$. Thus, the complete expressions for the $t_{\text {op }}$ and $t_{\mathrm{wp}}$ time delays are

$$
t_{w p}=\frac{L W \cdot \cos \phi-L M \cdot \sin \phi}{v_{c}} \cdot \cos \Phi
$$

and

$$
t_{e p}=\frac{L E \cdot \cos \phi-I M \cdot \sin \phi}{V_{C}} \cdot \cos \Phi
$$

where $L E=576 \mathrm{~m}, L M=47 \mathrm{~m}$, and $L W=128 \mathrm{~m}$. Note, that the sign of $t_{\text {op }}$ and $t_{\mathrm{wp}}$ can be negative. The negative sign means the lightning EM wave has arrived at the east or west pole, respectively, before it has arrived at the loops. A third equation can also be obtained by taking the difference of Eq. (2.3) from Eq. (2.4) to obtain the time delay between 
the east and west ends of the line. However, this equation is obviously not independent. The redundant equation is

$$
t_{a}=t_{e p}-t_{w D}=\left(\ell / v_{c}\right) \cos \phi \cos \phi
$$

where $\ell$ is the length of the power line. Two of the above three equations have to be solved simultaneously in order to obtain the lightning azimuth and elevation from measured time delays. Solving eqs.(2.4) and (2.5), we obtain the following expression for $\phi$ and $\Phi$ in terms of the delay times and known distances

$$
\phi=\tan ^{-1}\left[\frac{L E}{I M}-\frac{\ell \cdot t_{e p}}{I M \cdot t_{a}}\right]
$$

and

$$
\Phi=\cos ^{-1}\left[\frac{v_{c} \cdot t_{a}}{1 \cdot \cos \phi}\right]
$$

Note, that Eq. (2.6) suggests that a supplementary angle to $\phi$ will also satisfy the equation. However, the correct azimuth angle can be chosen since we know the sign of the delay times $t_{\text {op }}$ and $t_{\mathrm{wp}}$. In other words, the sign of the time delay, $t_{a}$, gives us the general direction (east or west of the power line) from which the lightning EM wave strikes the power line. To show the previous point graphically, plots of $t_{\text {op }}$ and $t_{\text {wp }}$ versus the azimuth, $\phi$, are shown for zero elevation angle in Figure 2.4 and Figure 2.5, respectively. In Figure 2.4 for $t_{\text {op }}$ equal to $+1.90 \mu \mathrm{sec}, \phi$ is equal to $0^{\circ}$, whereas for $t_{\text {ep }}$ equal to $-1.90 \mu \mathrm{sec}, \phi$ is equal to $180^{\circ}$. Similarly, in Figure 2.5 , for $t_{\mathrm{wp}}$ equal to $+0.42 \mu \mathrm{sec}, \phi$ is equal to $0^{\circ}$, whereas for $t_{\mathrm{wp}}$ equal to $-0.42 \mu \mathrm{sec}, \phi$ is equal to $180^{\circ}$. 
EAST POLE TIME DELAY

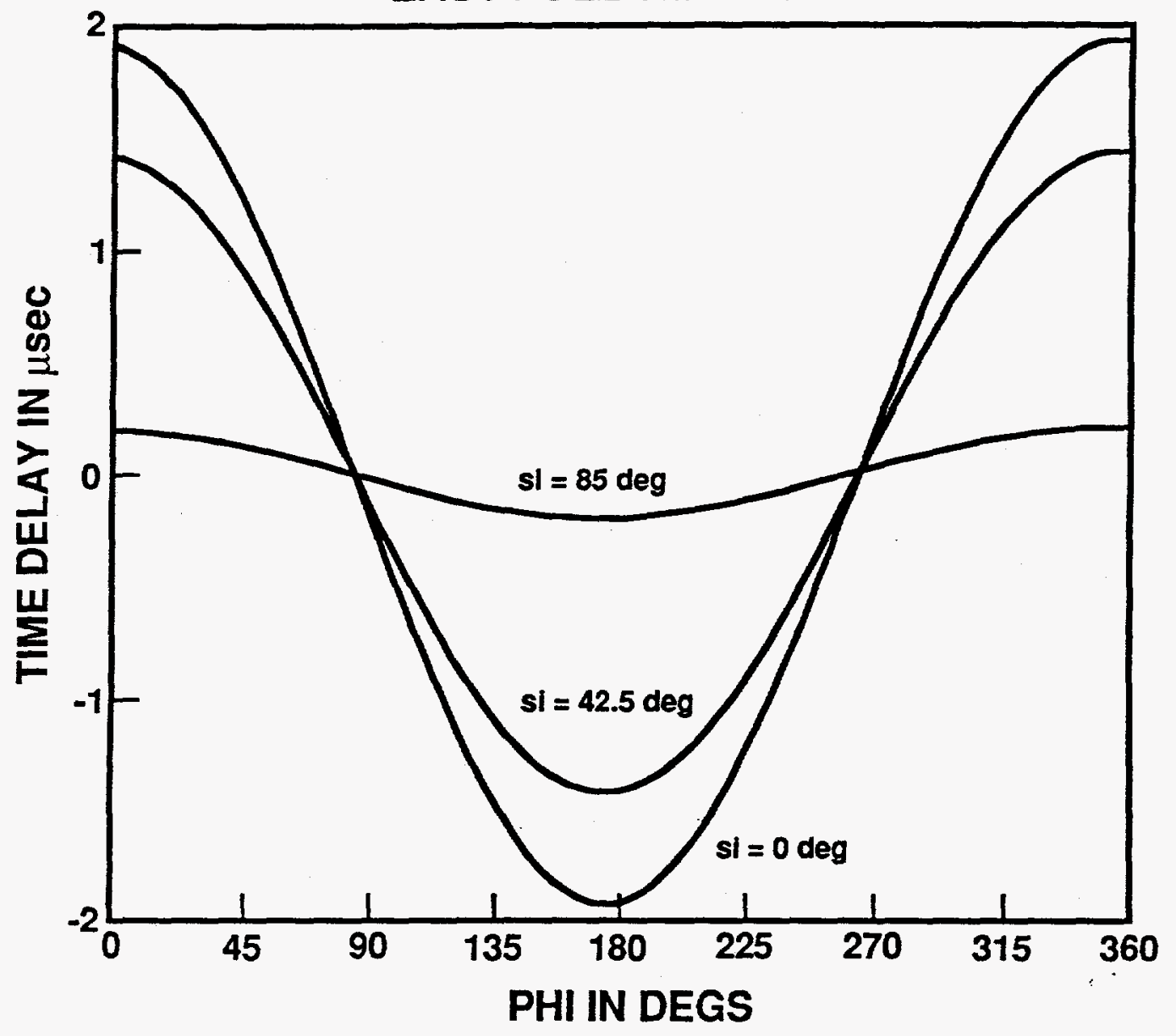

Fig. 2.4 East pole time delay as a function of azimuth and elevation. Only three values of elevation angles are used: $\Phi=0^{\circ}, 42.5^{\circ}$, and $85^{\circ}$. 


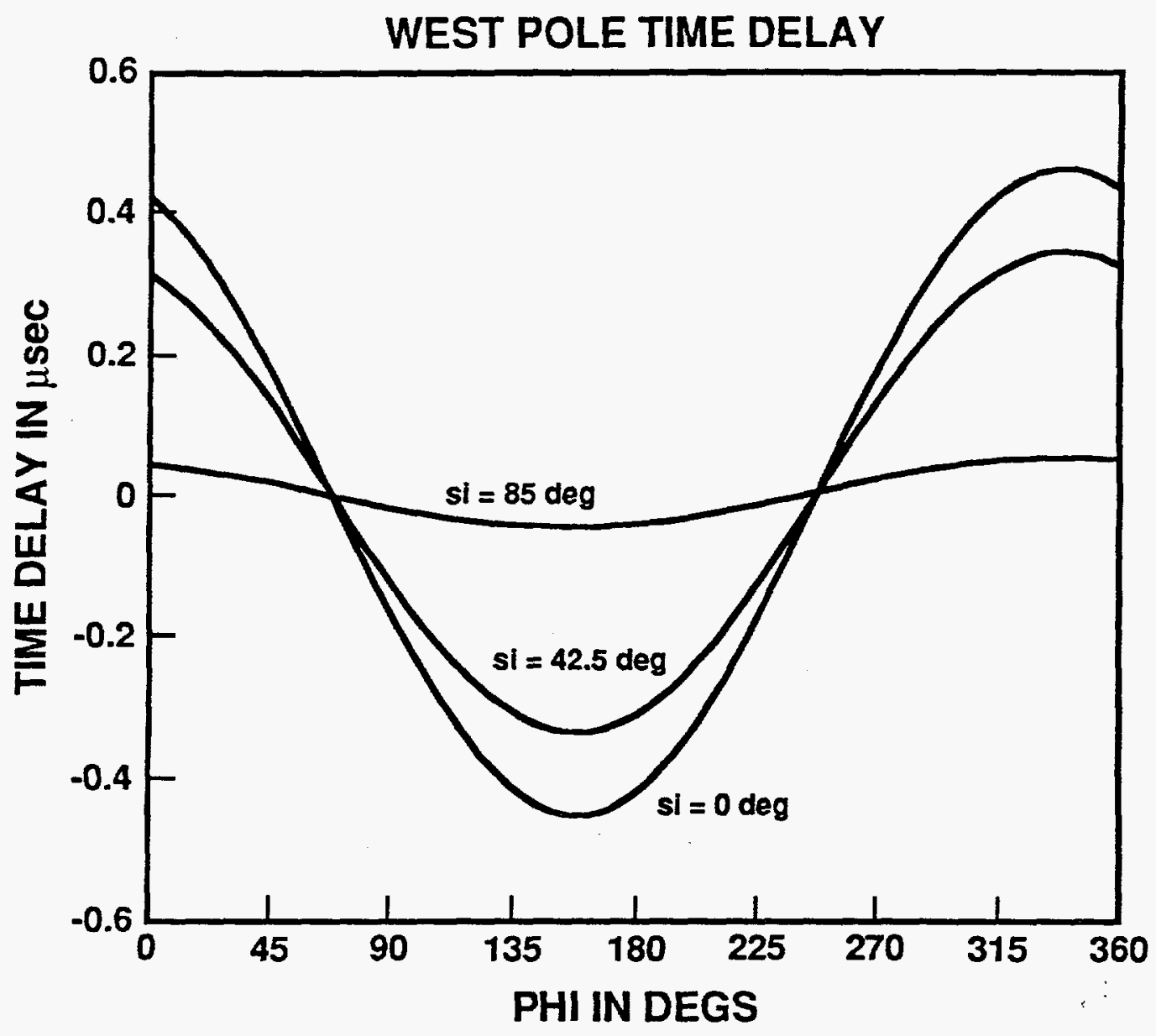

Fig. 2.5 West pole time delay as a function of azimuth and elevation. Only three values of elevation angles are used: $\Phi=0^{\circ}, 42.5^{\circ}$, and $85^{\circ}$. 
17

Also note that Eq. (2.7) suggests the negative of the elevation angle, $\Phi$, can also satisfy the equation. However, the physical layout of the experiment does not allow any negative values for the elevation angle.

A sensitivity analysis of the time delay method, shows that, due to the errors involved, it cannot yield detailed information but rather a rough estimate of the azimuth region of the incident electric field.

Figure 2.6 shows typical examples of measured magnetic fields from five overhead lightning events to be used as inputs to the program EMPIIN that calculates line voltages. The calculated voltages are given and compared to measured voltages in Chapter 8 . 
Event:

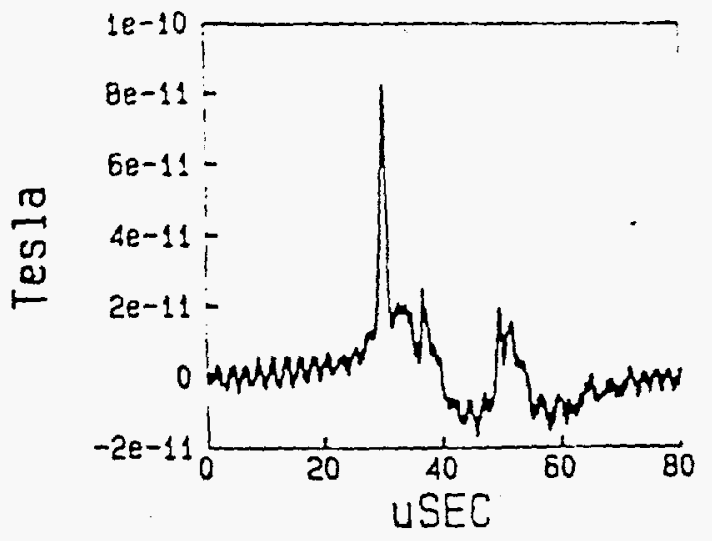

Event19

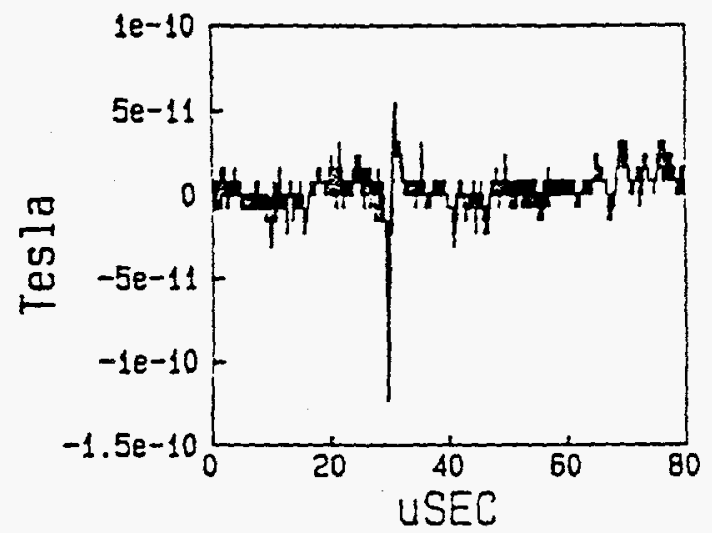

Event31

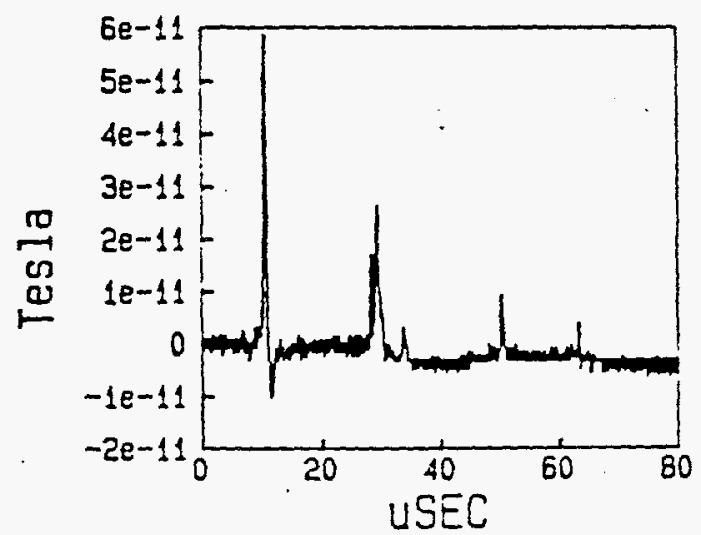

Eventz2

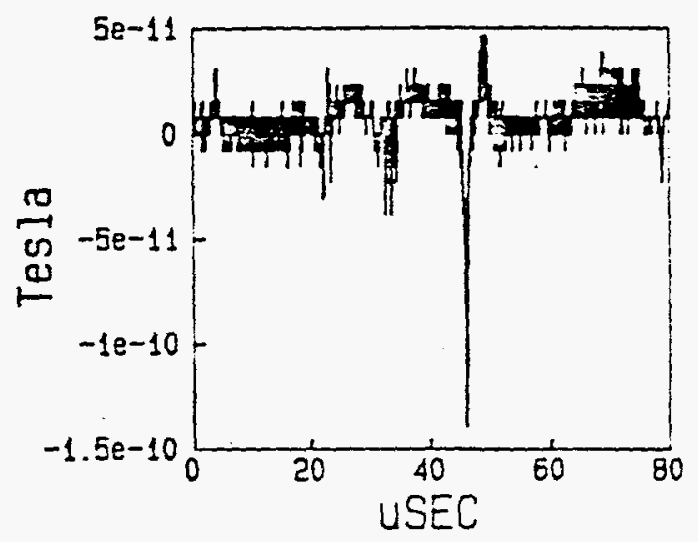

Event40

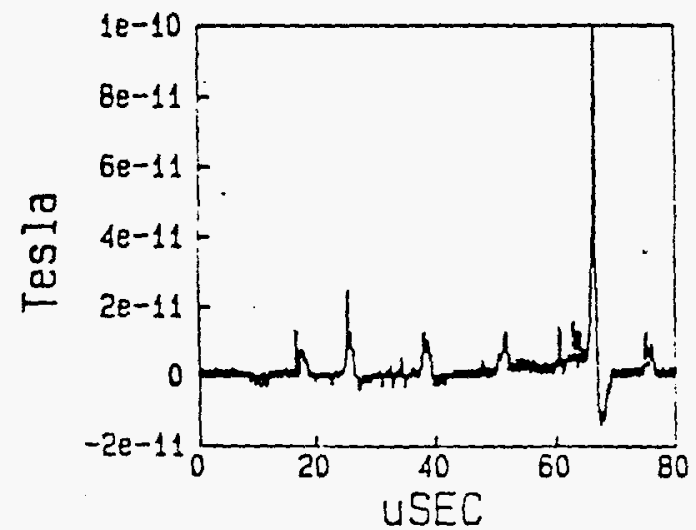

Fig. 2.6 Measured north-south component of the magnetic field for five overhead lightning events. 
CHAPTER III

\section{CHARACTERIZATION OF IIGHTNING INDUCED VOLTAGES AND INDUCING FIELDS FROM VERY CLOSE ARTIFICIAIIY INITIATED IIGHTNING}

\section{$\underline{3.1}$ Introduction}

Eleven strokes from three artificially initiated lightning flashes, one seven-stroke flash on day 232, and two two-stroke flashes on day 240 , were recorded at the Kennedy Space Center during the summer of 1986 . In this chapter, we characterize the vertical electric fields measured 500 m away from the artificially initiated lightning discharge and the voltages measured at either end of the test line for the 11 strokes. The data shown were obtained by digitizing magnetic tape records from the direct and FM channels of an analog tape recorder.

\subsection{Vertical Electric Fields}

The vertical electric field changes associated with the 7-stroke triggered lightning with two 2-stroke triggered lightnings measured about $500 \mathrm{~m}$ away from the artificial lightning triggering site, are shown in Figures $3.1,3.2$, and 3.3 . The waveforms in these figures have a $5 \mathrm{msec}$ decay time due to the electronics used. That is, if the waveform were a step function, it would exponentially decay with a $5 \mathrm{msec}$ time constant rather than remain constant after the step. The individual return stroke vertical electric field changes, together with voltages measured at either end of the test distribution line are shown in Figures 3.5 through 3.7. To facilitate the characterization of the return stroke fields, a typical return stroke field has been sketched in Figure 3.4 , in which all the parameters of interest (defined below) have been identified. 


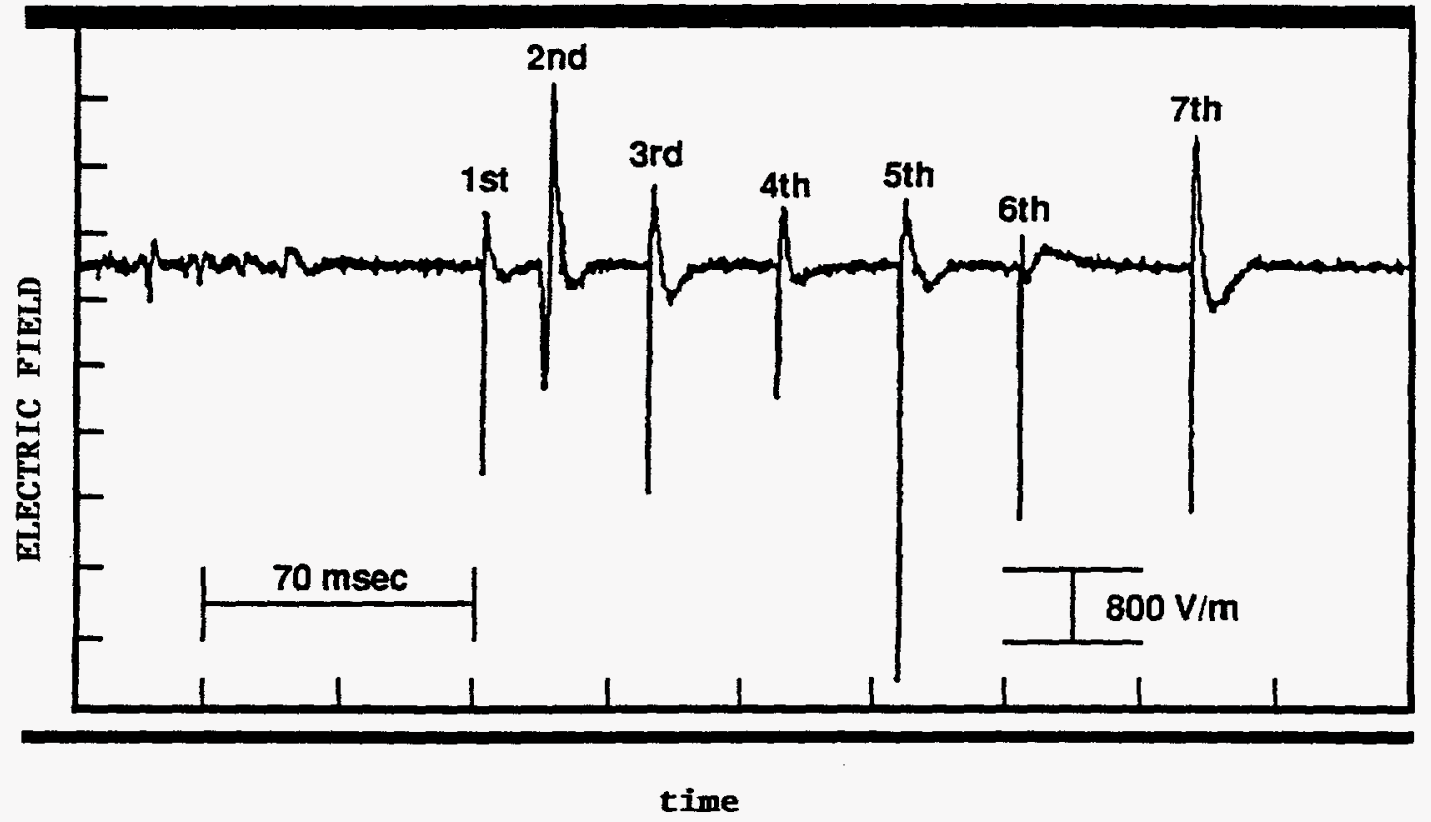

Fig. 3.1 Vertical electric field intensity associated with flash 1 measured $500 \mathrm{~m}$ away from artificially initiated lightning on day 232 at Kennedy space Center, Florida in 1986. The system decay time is $5 \mathrm{msec}$. 


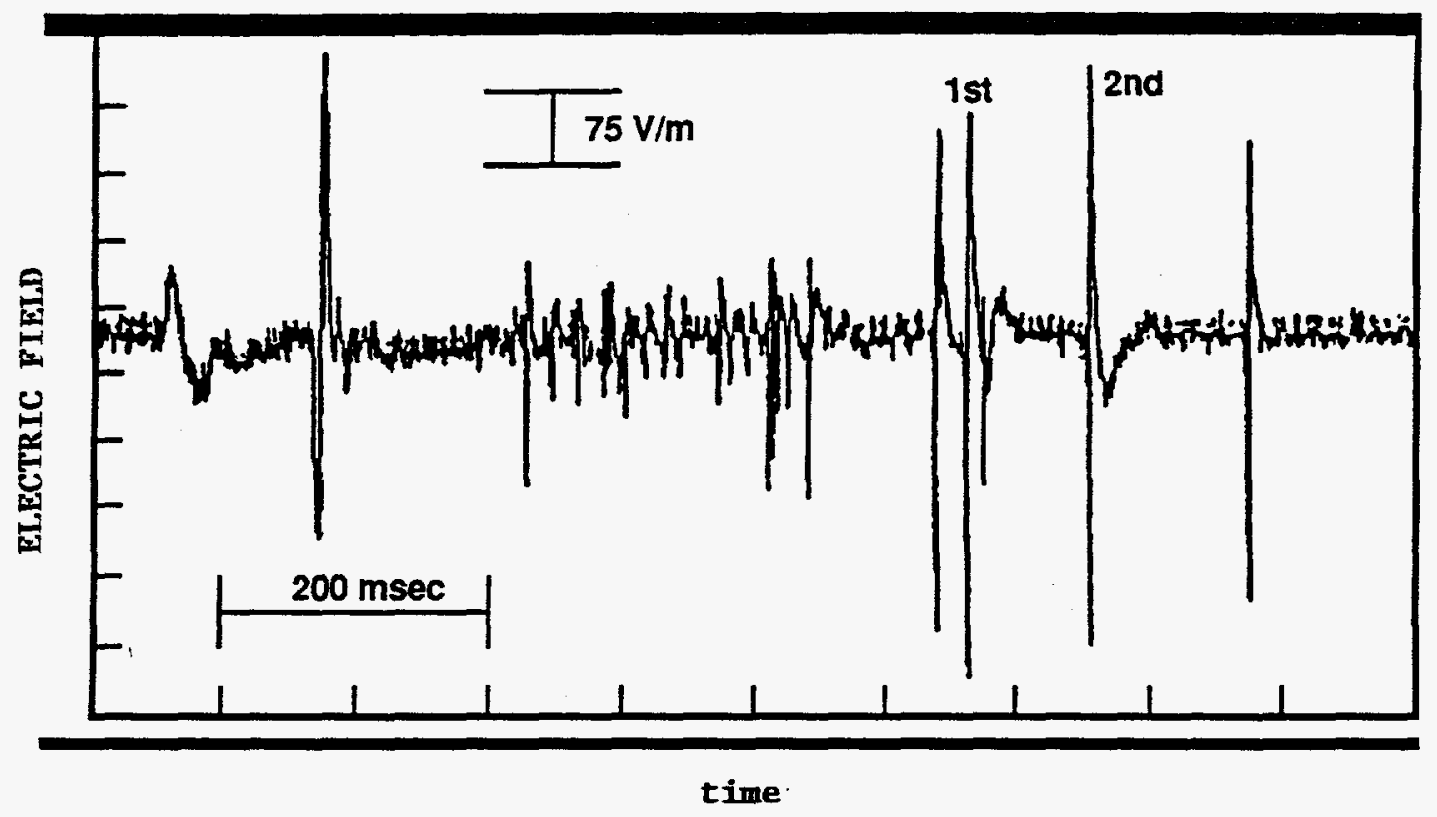

Fig. 3.2 Vertical electric field intensity associated with flash 1 measured $500 \mathrm{~m}$ away from artificially initiated lightning on day 240 at Kennedy space Center, Florida in 1986. The system decay time is $5 \mathrm{msec}$. The pulses preceding the first stroke and the one after the second stroke have rise times of the order of tens of microseconds and hence are not return strokes. 


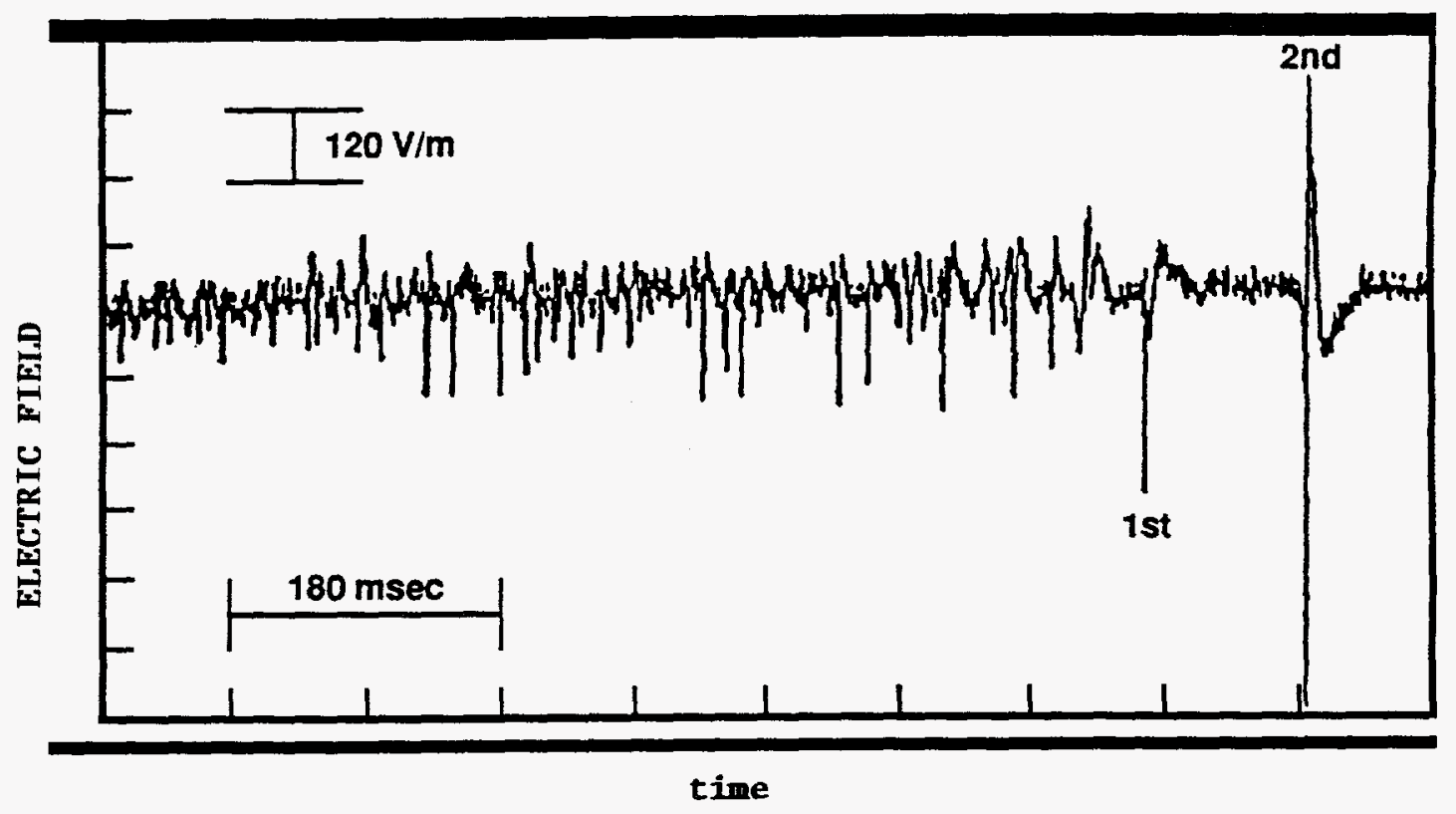

Fig. 3.3 Vertical electric field intensity associated with flash 2 measured $500 \mathrm{~m}$ away from artificially initiated lightning on day 240 at Kennedy Space Center, Florida in 1986. The system decay time is $5 \mathrm{msec}$. 


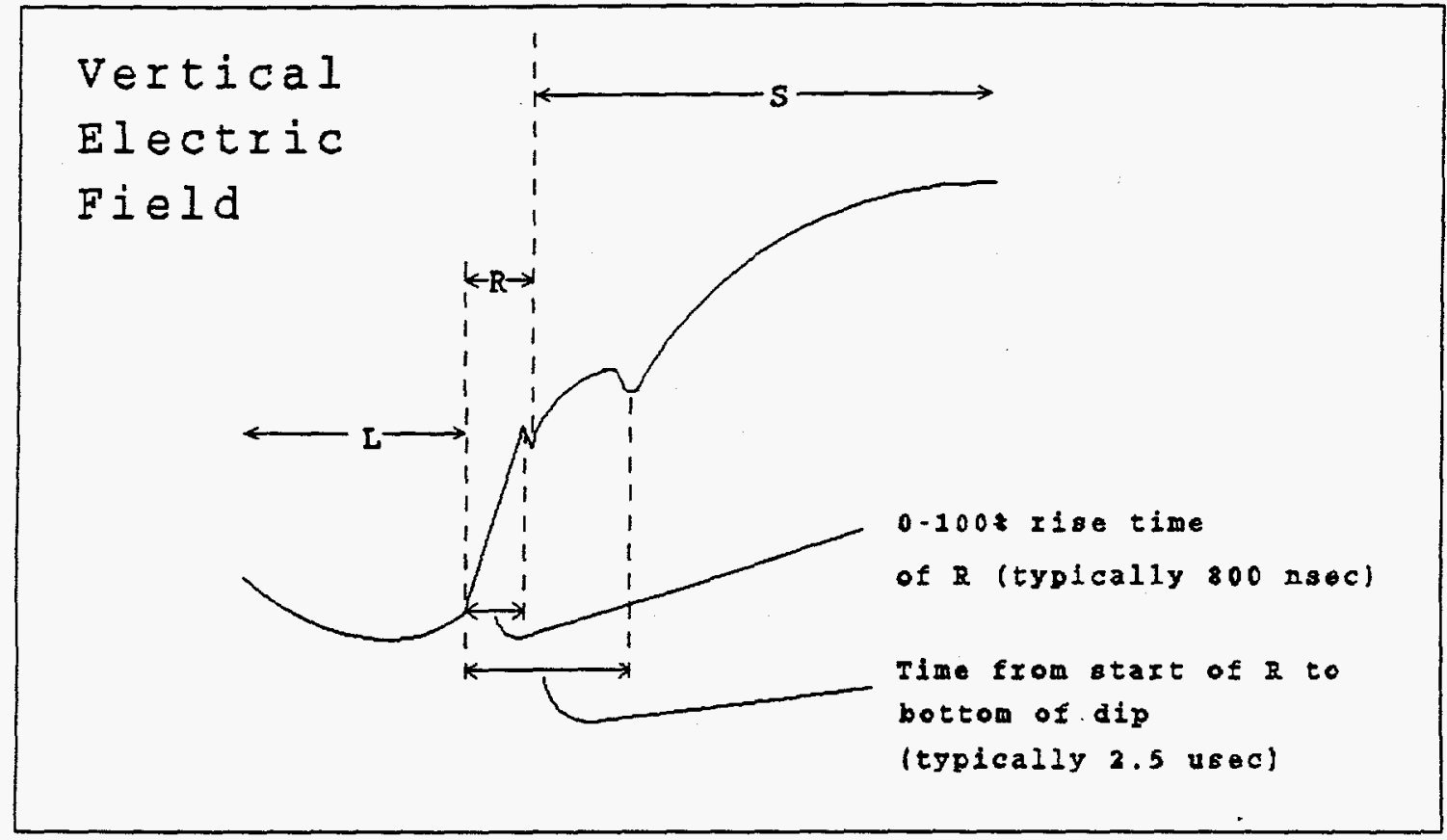

Not to scale

Fig. 3.4 A sketch of the vertical electric field intensity for a typical return stroke showing definitions of some salient parameters. 
(a)

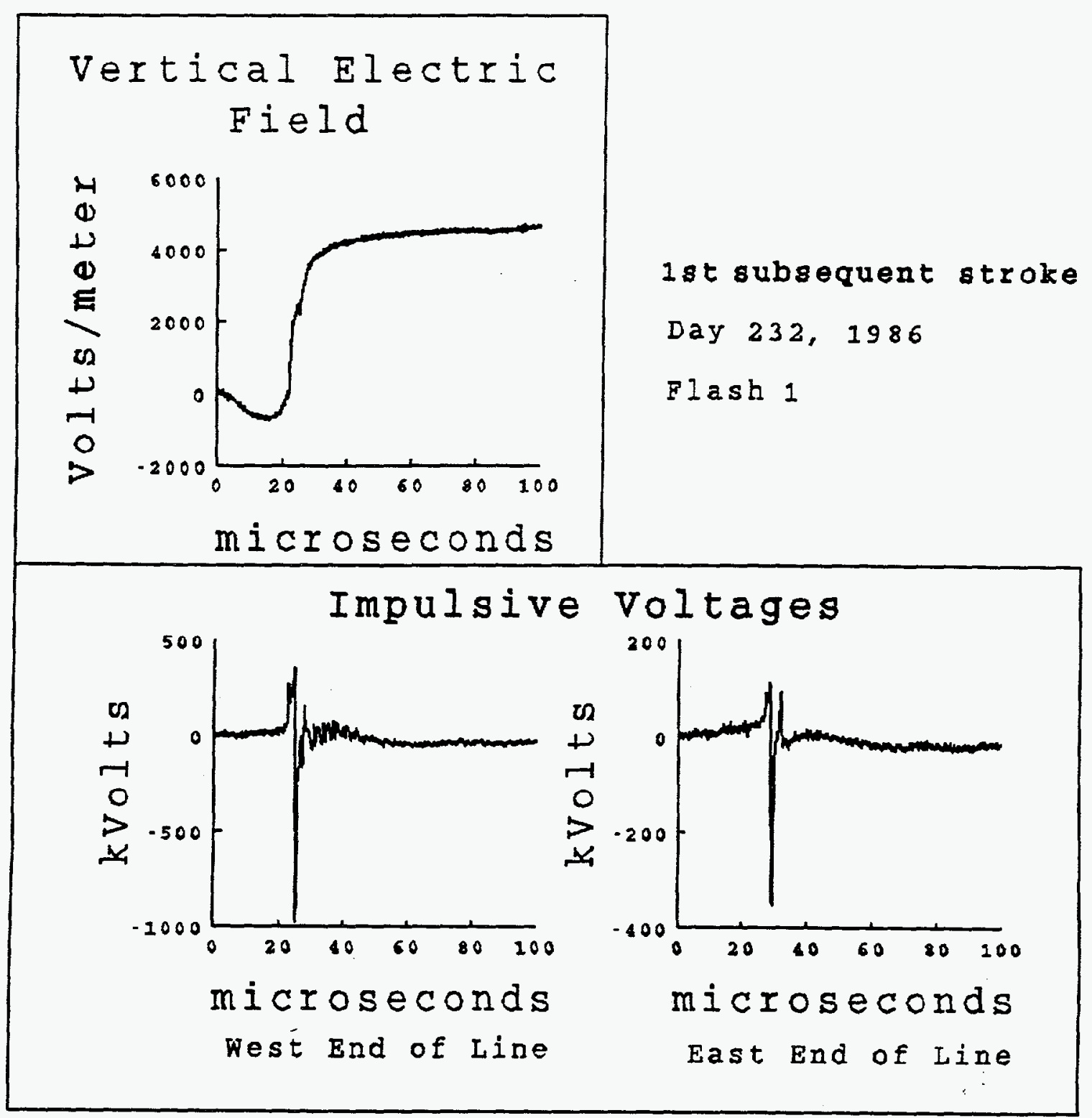

Fig. 3.5 Return stroke vertical electric field waveforms measured 500 $\mathrm{m}$ away from artificially initiated lightning and voltages at either end of the test overhead line, one end located $20 \mathrm{~m}$ from the discharge, for lightning flash 1 on day 232 at Kennedy Space Center, Florida in 1986. (a) through (g) represent the seven individual strokes recorded for that flash (see Figure 3.1). The terms oscillatory voltage and impulsive voltage are discussed in the text. 


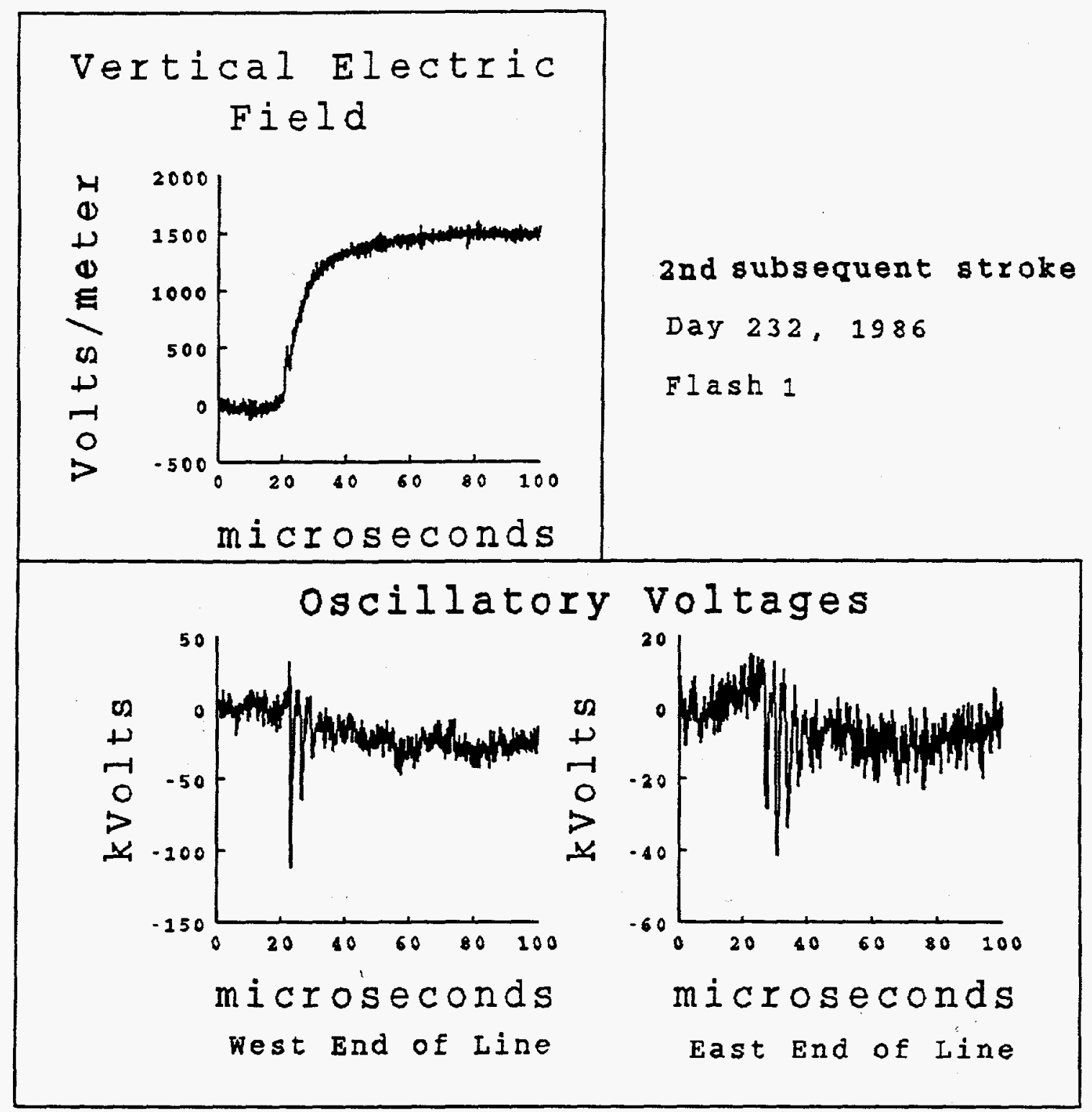

Figure 3.5 continued 


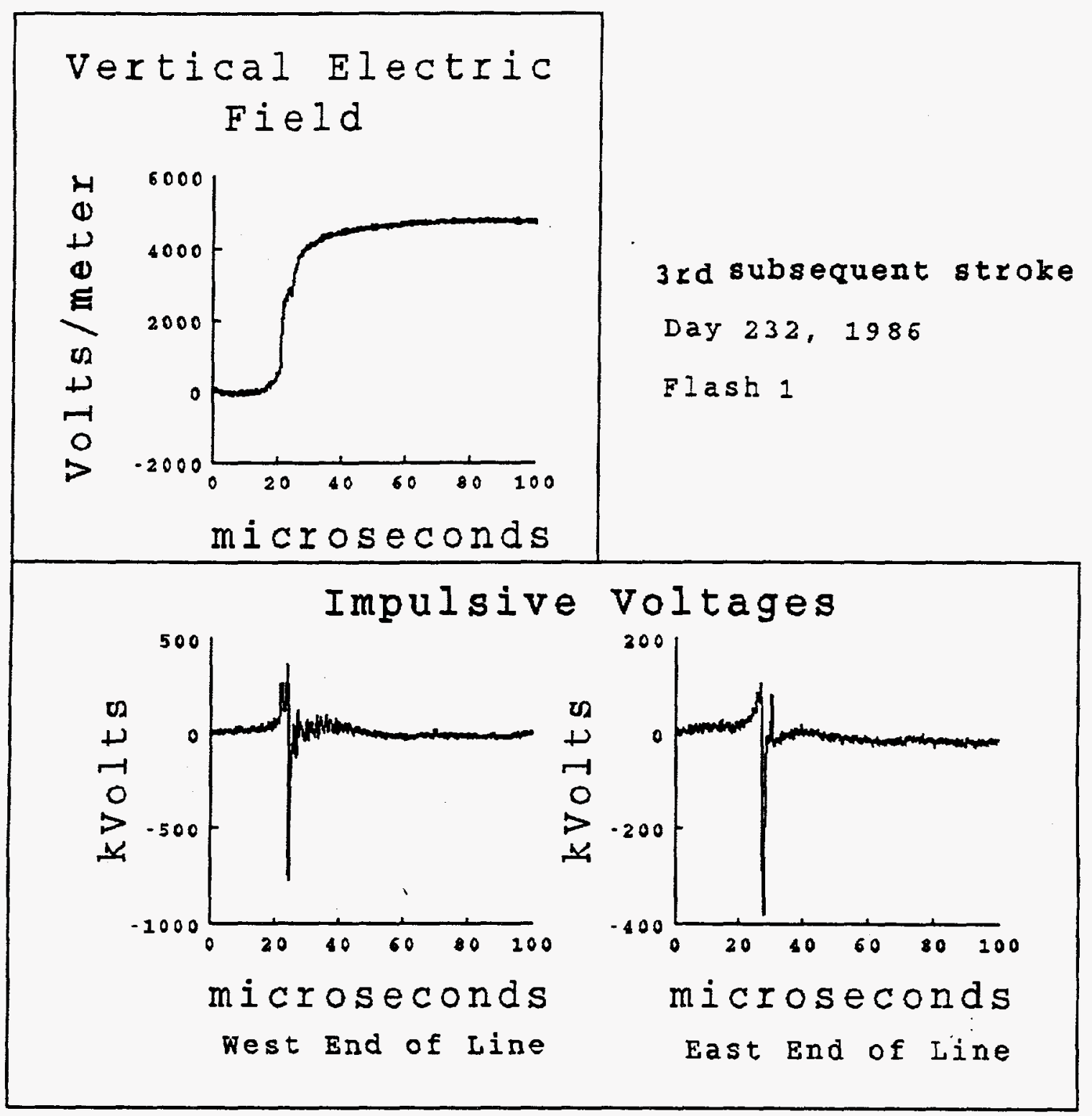

Figure 3.5 continued 


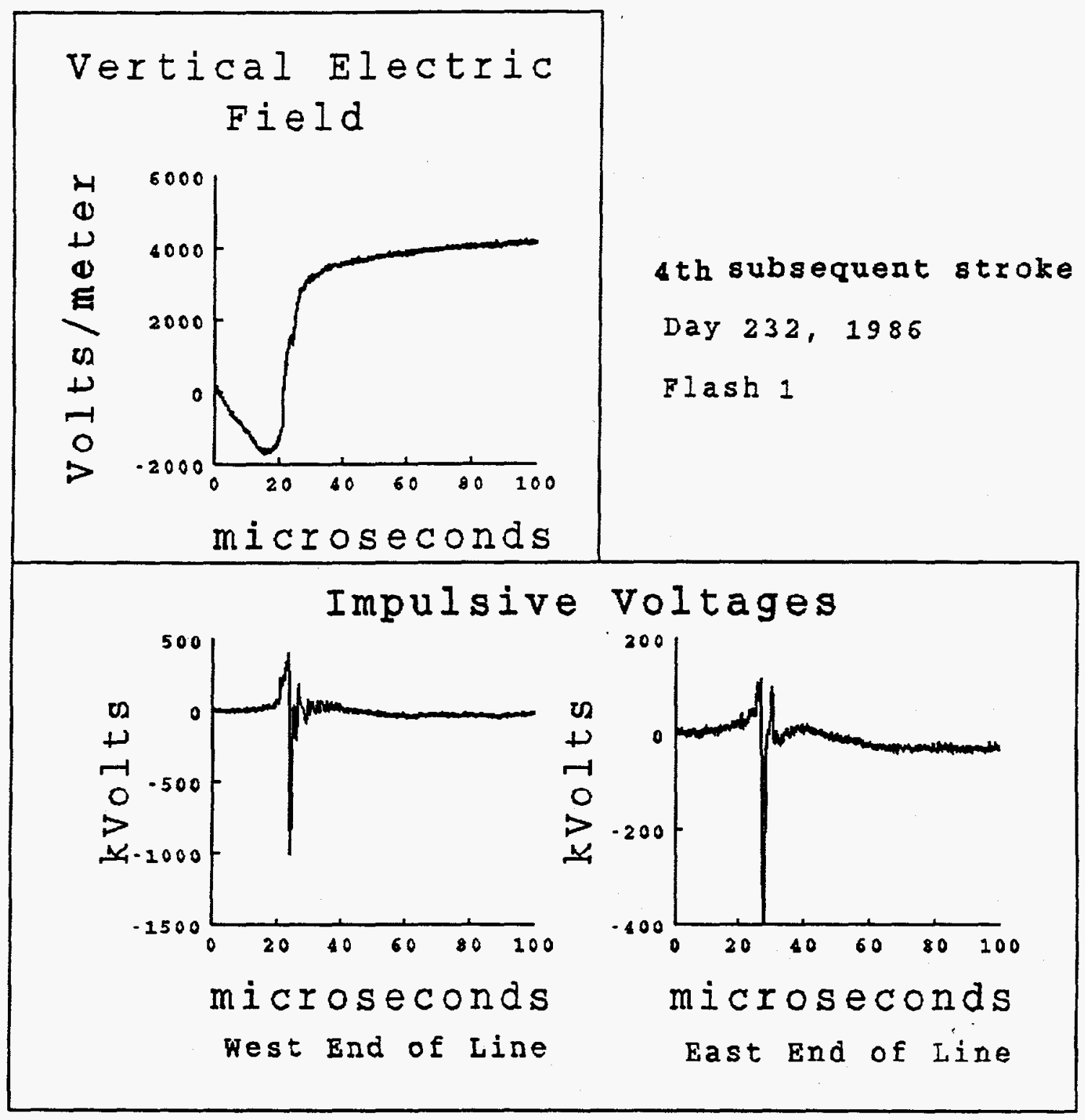

Figure 3.5 continued 
(e)

Vertical Electric Field

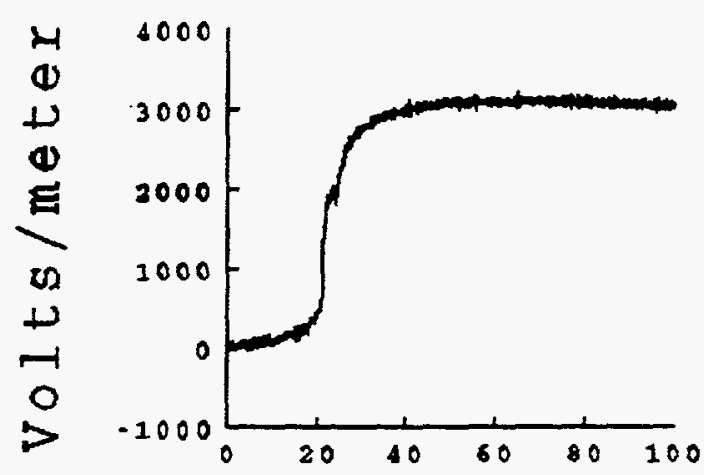

5 th subsequent stroke Day 232, 1986

Flast 1

microseconds

Impulsive Voltages
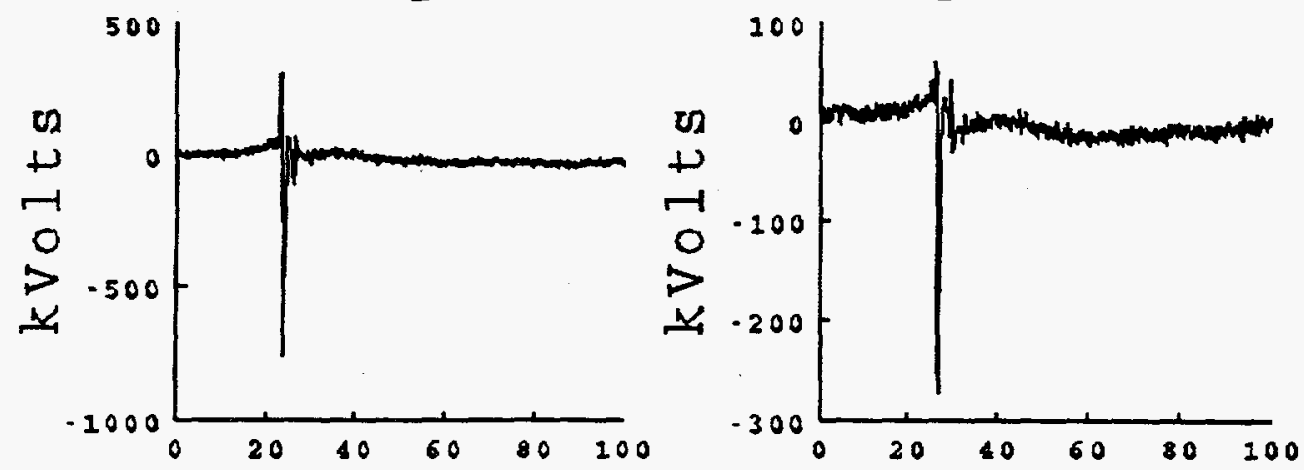

microseconds west End of Line microseconds East End of Line

Figure 3.5 continued 


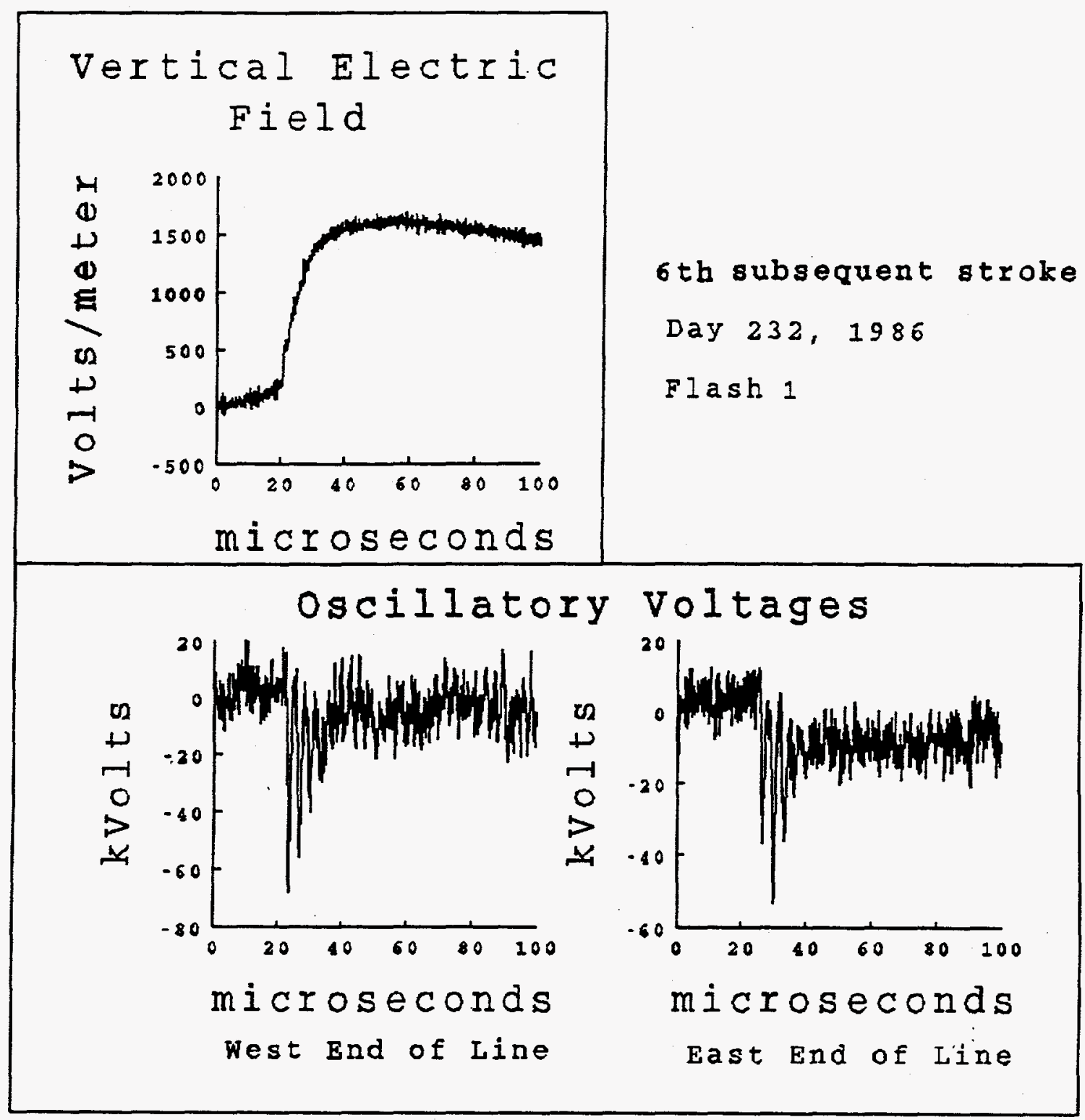

Figure 3.5 continued 


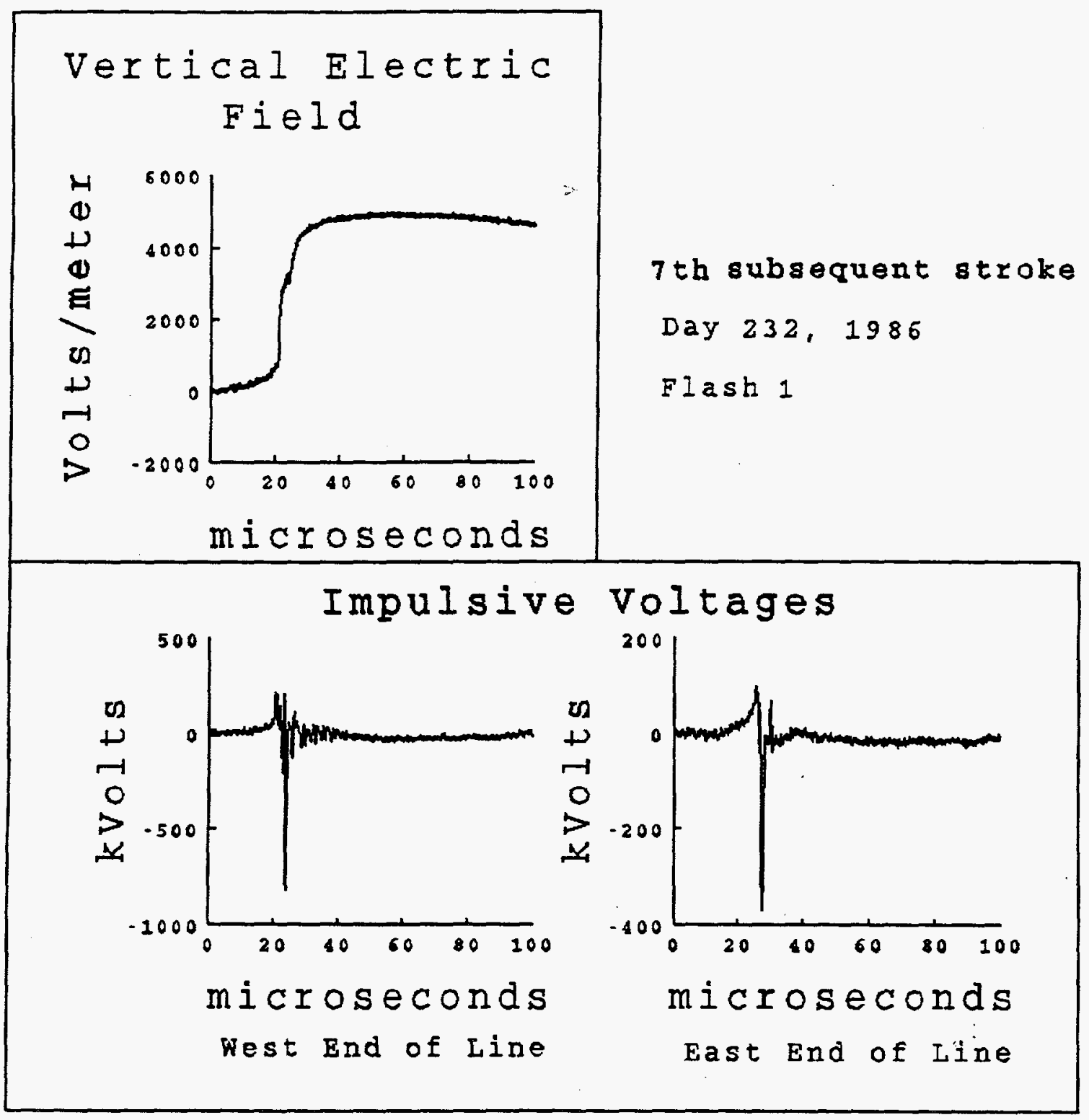

Figure 3.5 continued 
(a)

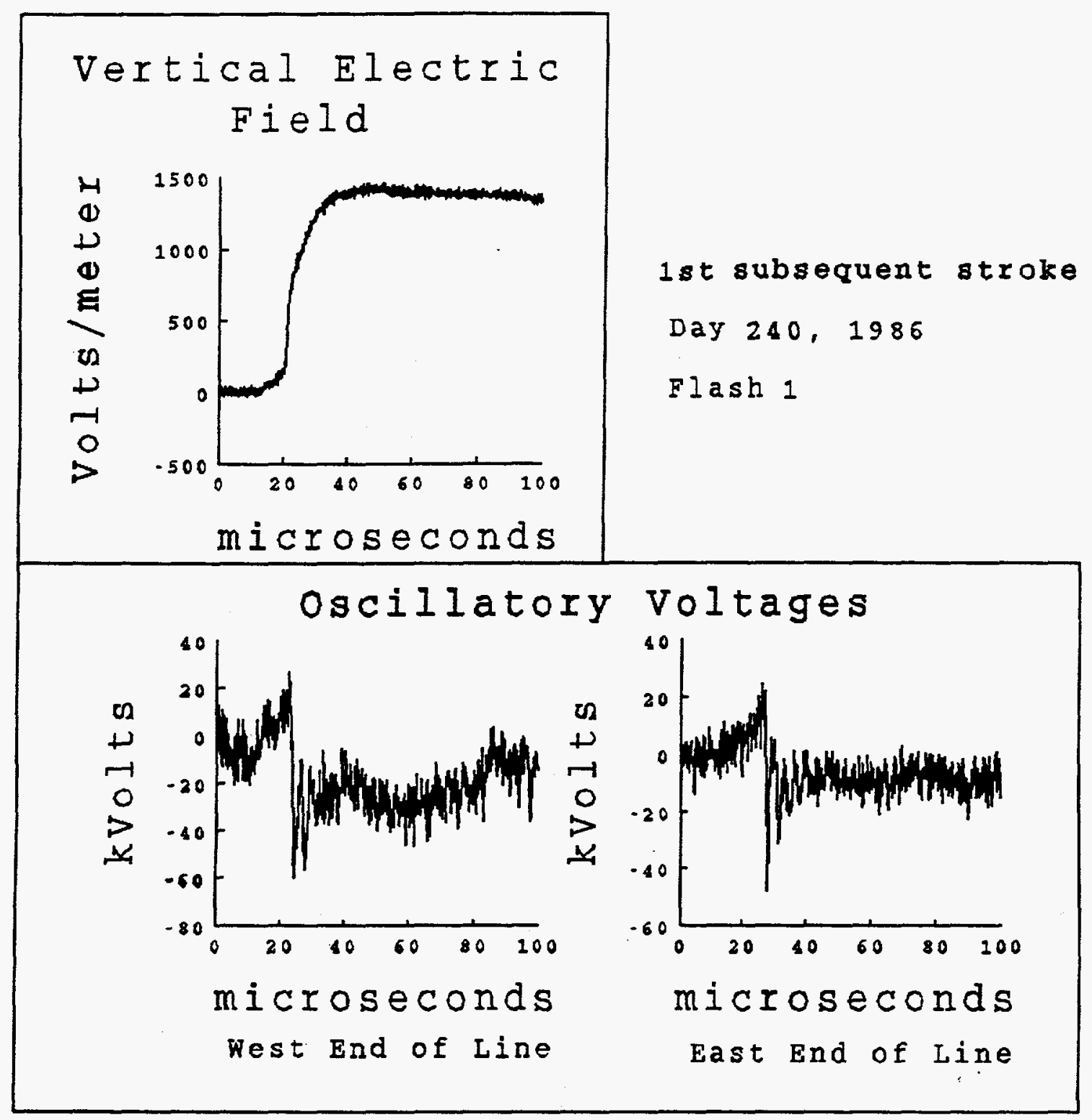

Fig. 3.6 Return stroke vertical electric field waveforms measured 500 $\mathrm{m}$ away from artificially initiated lightning and voltages at either end of the test overhead line, one end located $20 \mathrm{~m}$ from the discharge, for lightning flash 1 on day 240 at Kennedy Space Center, Florida in 1986. (a) and (b) represent the two individual strokes recorded for that flash (see Figure 3.2 ). The terms oscillatory voltage and impulsive voltage are discussed in the text. 
(b)

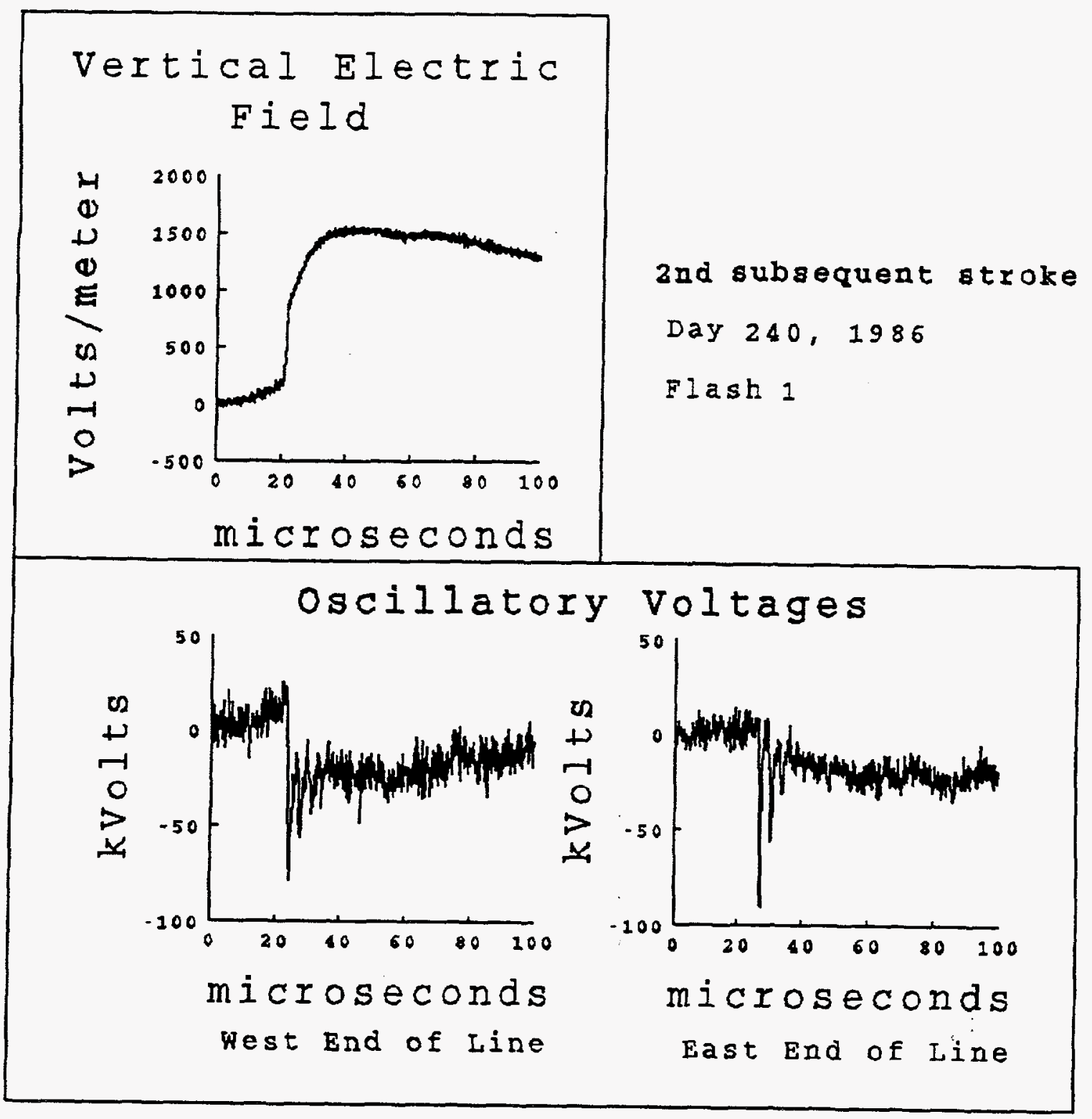

Figure 3.6 continued 
(a)

Vertical Electric Field

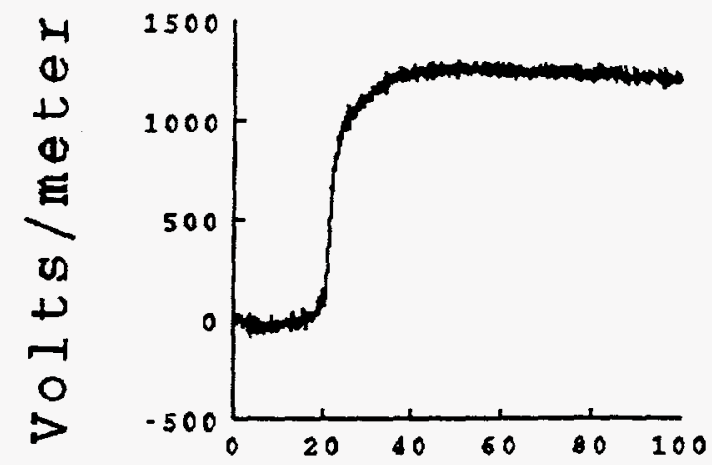

microseconds

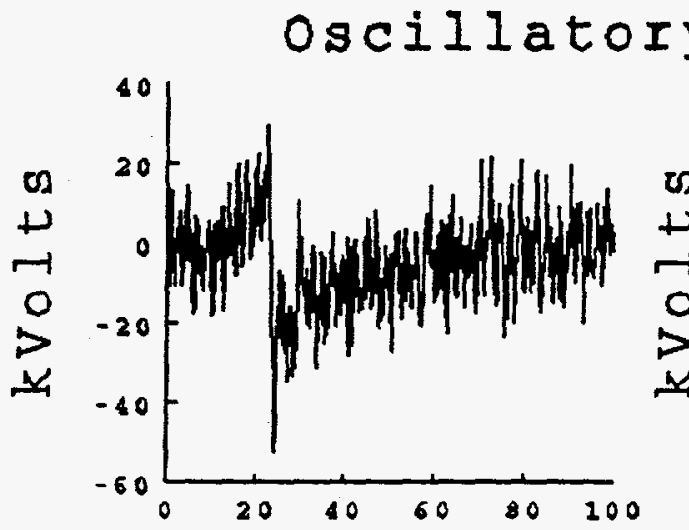

microseconds west End of Line

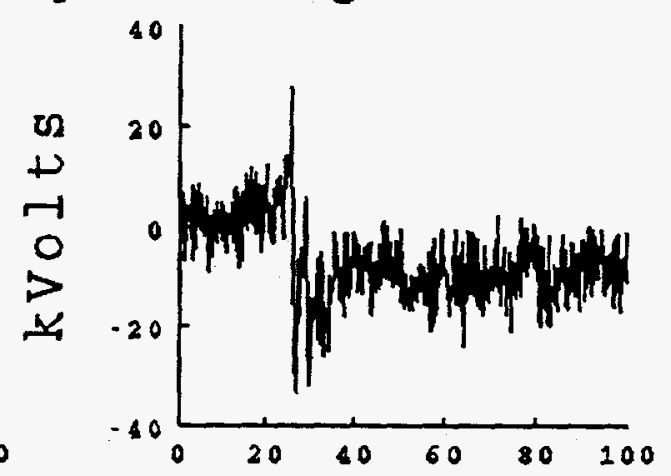

microseconds

East End of Line

Fig. 3.7 Return stroke vertical electric field waveforms measured 500 m away from artificially initiated lightning and voltages at either end of the test overhead line, one end located $20 \mathrm{~m}$ from the discharge, for lightning flash 2 on day 240 at Kennedy Space Center, Florida in 1986. (a) and (b) represent the two individual strokes recorded for that flash (see Figure 3.3 ). The terms oscillatory voltage and impulsive voltage are discussed in the text. 
(b)

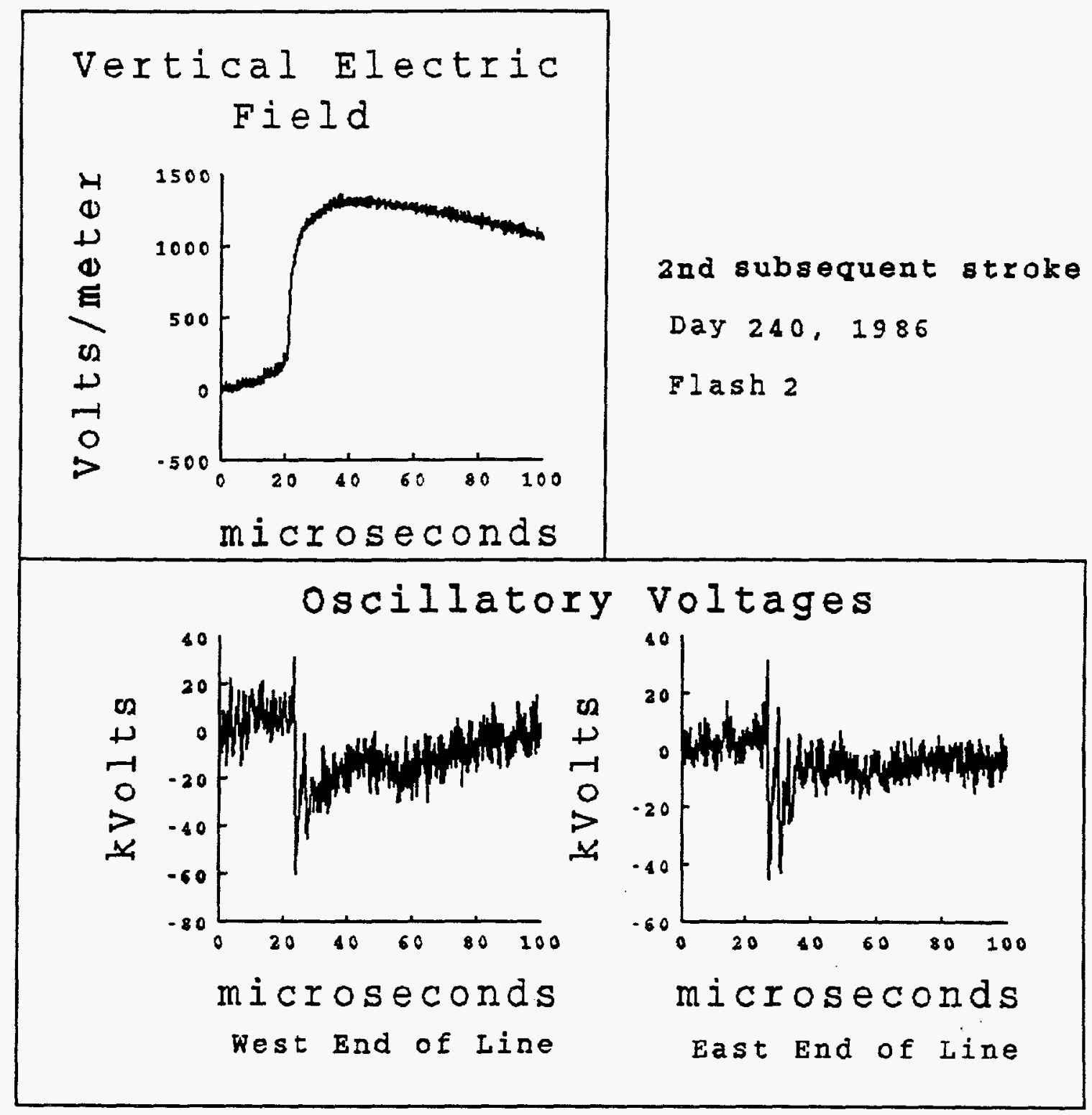

Figure 3.7 continued. 
the dart-leader. This slow change, which we label $\mathrm{L}$ for leader, precedes a fast positive field change termed $R$, for return stroke, which ends typically in a small glitch (not discernible in Figures 3.5 through 3.7). This fast change is followed by a slower change of the same polarity termed $S$ in Figure 3.4. The overall $S$ change, which typically exhibits a dip near its onset (which can be seen in Figures 3.5 through 3.7 and which is not to be confused with the small glitch at the end of R), rises with constantly decreasing slope. Some parameters measured for each electric field waveform are presented in Table 3.1. The average amplitude of the $R$ change is $878 \mathrm{~V} / \mathrm{m}$ with a standard deviation of $445 \mathrm{~V} / \mathrm{m}$. The average 0 to 1008 risetime of $R$ is 803 nsec with a standard deviation of $337 \mathrm{nsec}$. The average time between the start of $R$ and the bottom of the dip in the $S$ field change is $2.5 \mu$ sec with standard deviation $0.87 \mu s e c$.

\subsection{Induced Voltages}

The voltages measured at either end of the test line can be grouped into two categories: (1) those in which a series of evenly spaced, similarly shaped pulses with decaying amplitudes can be observed, which we define as "oscillatory" voltages, of which six were recorded, and (2) those that present a clearly dominant pulse with subsidiary oscillations of much lower amplitude (the largest subsidiary peaks being one fifth or less of the initial pulse amplitude), which we call "impulsive" voltages, and of which five were recorded. Voltage waveforms corresponding to impulsive and oscillatory voltages are shown in Figures 3.5 through 3.7 . For the discussion that follows, refer to Figures $3.8 \mathrm{a}$ and $3.8 \mathrm{~b}$ where we have sketched the characteristics of impulsive and oscillatory voltages and where we have included the definitions of the parameters to be discussed. Measurements of some salient features of the experimental voltage waveforms are found in tables 3.2 through 3.5 . Both oscillatory 
(a)

\section{IMPULSIVE VOLTAGES}

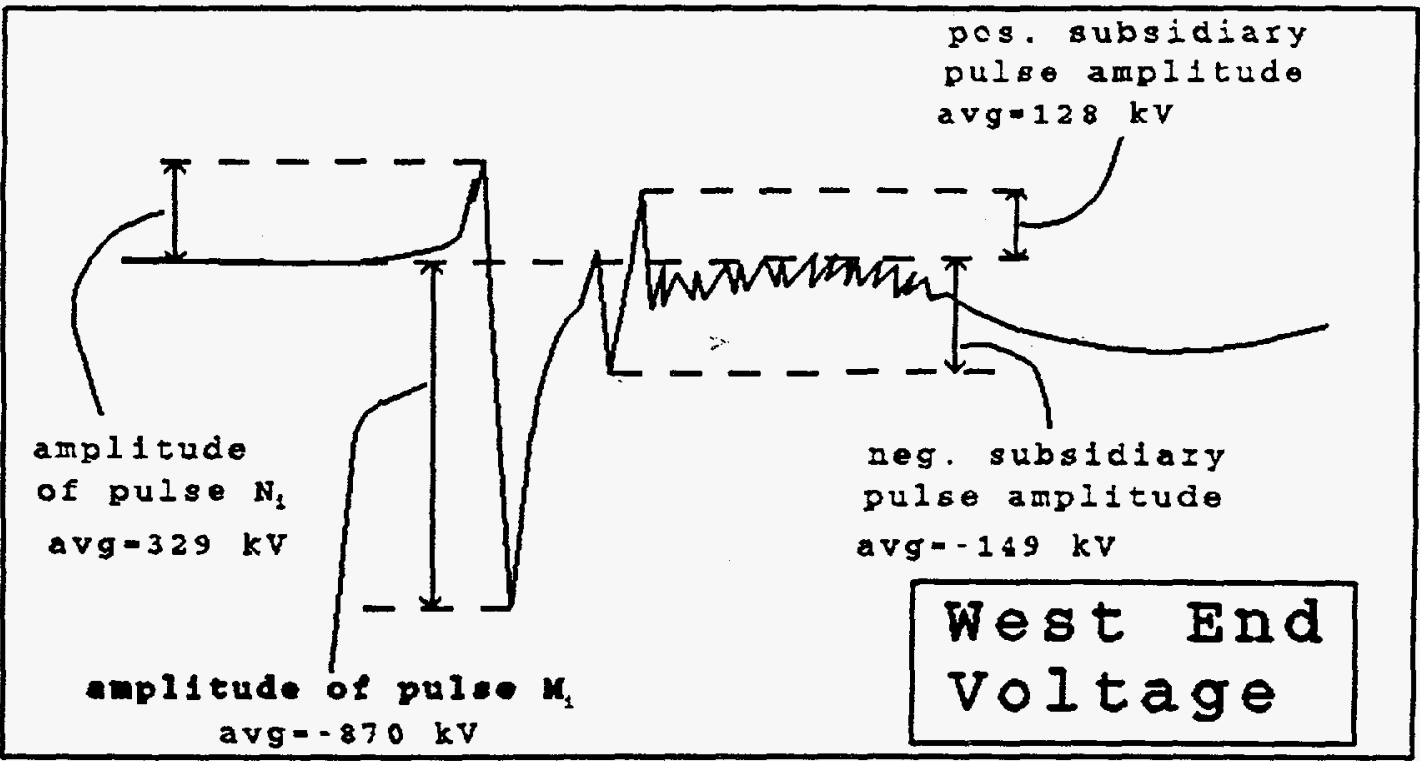

Not to scale

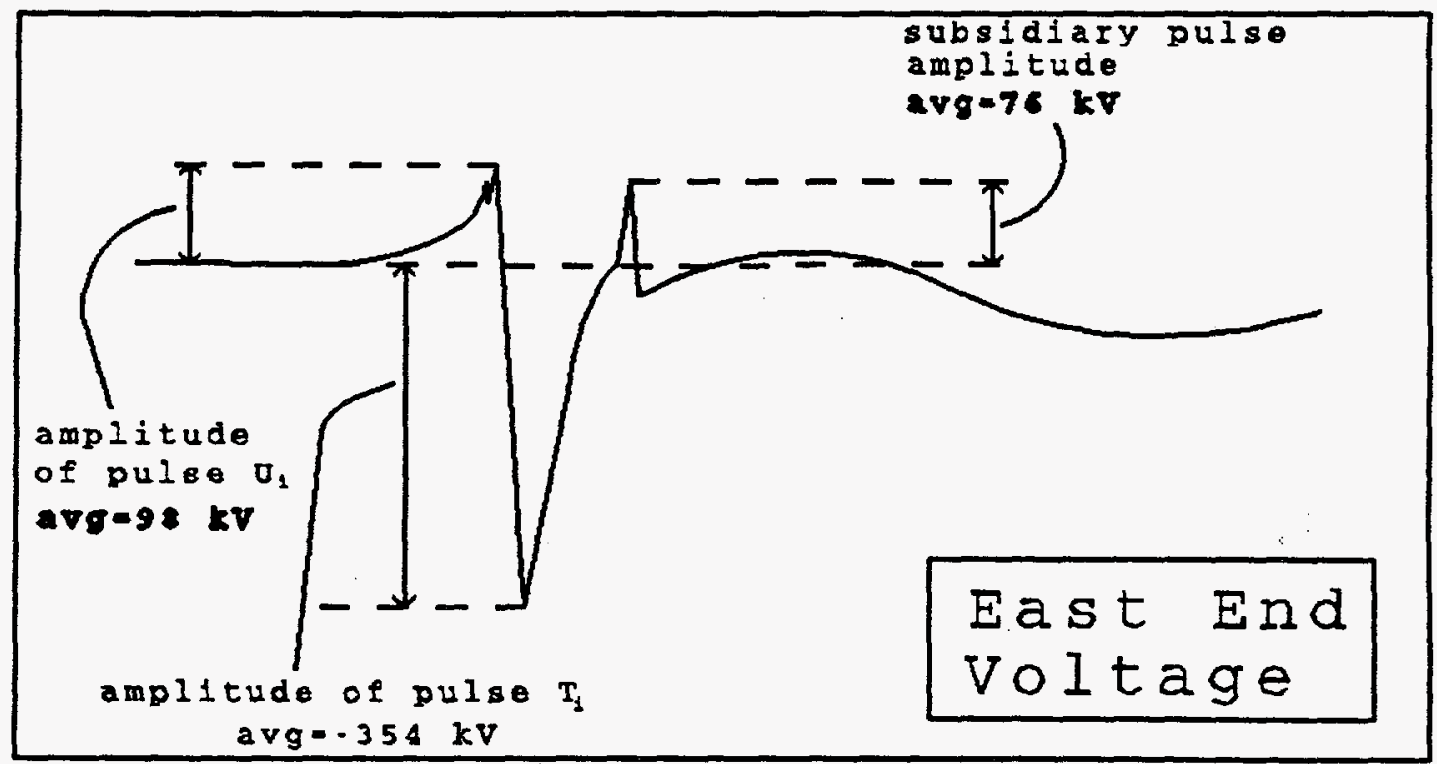

Not to scale

Fig. 3.8 Sketches of typical waveforms. (a) Impulsive voltages. Oscillatory voltages.

(b) 
(b)

\section{OSCILLATORY VOLTAGES}

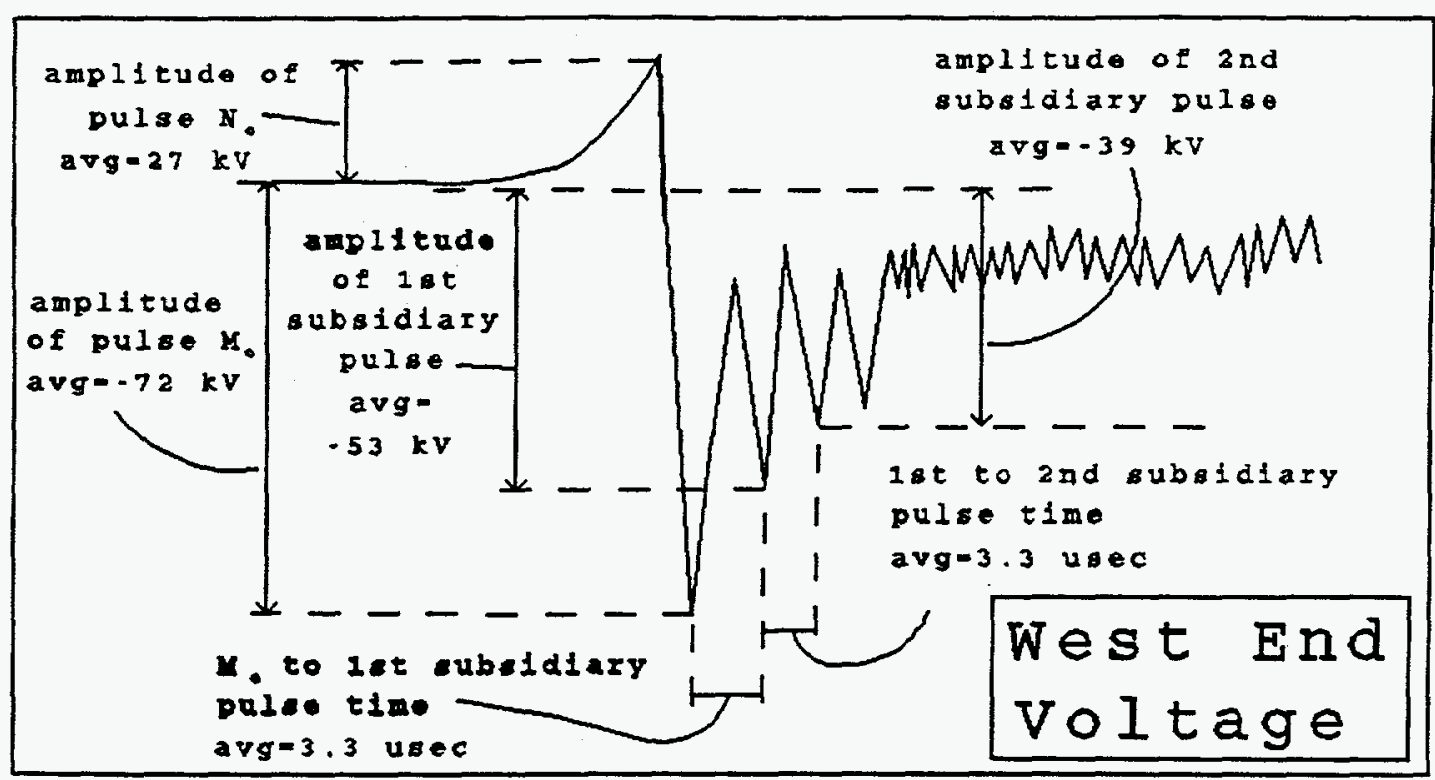

Not to scale

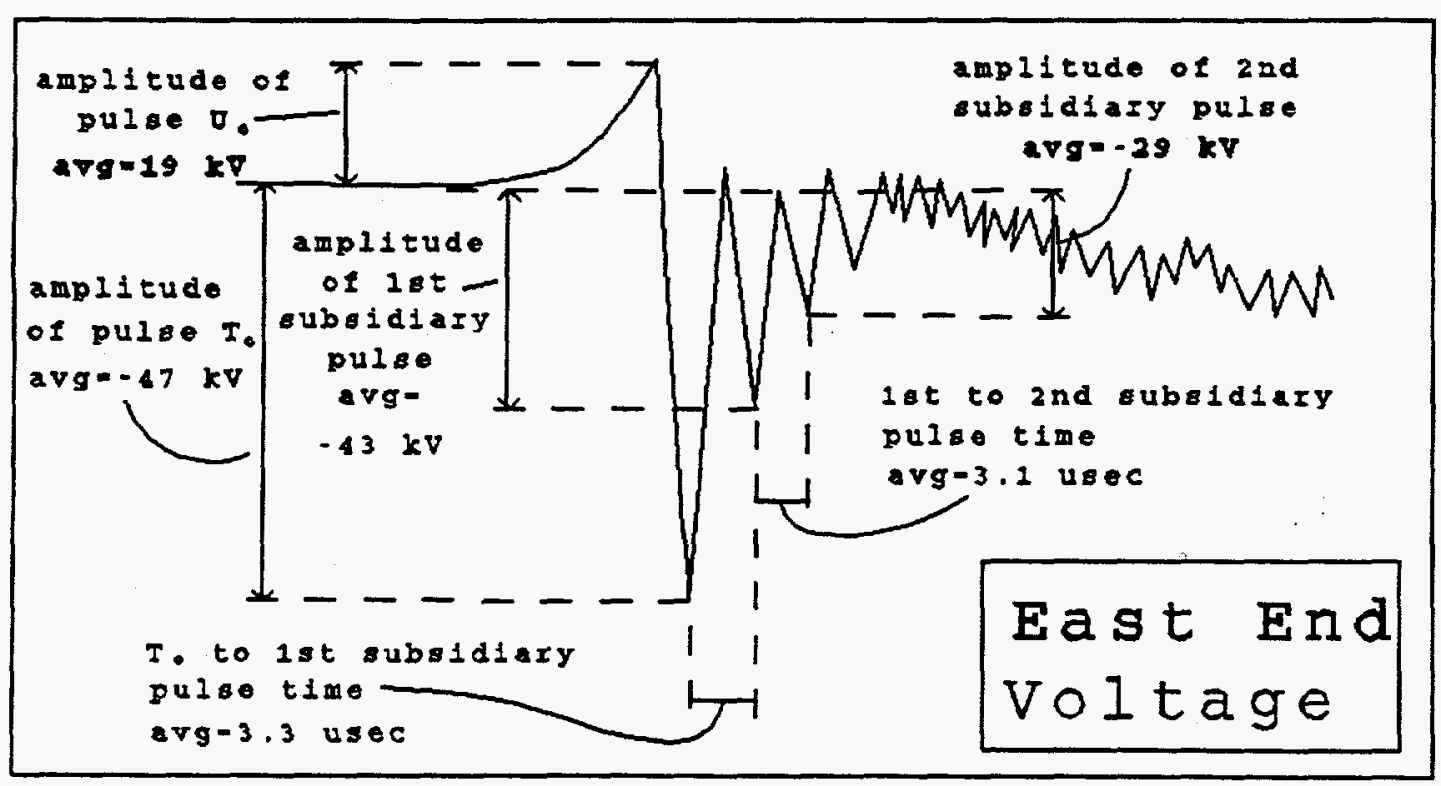

Not to scale

Figure 3.8 continued 
The vertical electric field starts with a slow field change presumably from and impulsive voltages were observed to occur for different strokes in the 7-stroke flash, whereas the two 2-stroke flashes produced oscillatory voltages only. The mean amplitude of the $R$ electric field changes associated with oscillatory voltages on the line, $500 \mathrm{v} / \mathrm{m}$, is less than half the mean amplitude of the $R$ changes associated with impulsive voltages, $1.33 \mathrm{kV} / \mathrm{m}$. As we shall see, the maximum induced oscillatory voltages are on average, a factor of 8 to 10 times less than the maximum impulsive voltages, indicating that there are other factors than the measured vertical field amplitude involved in the coupling.

Table 3.1. Amplitudes and risetimes of electric field $\mathbf{R}$ changes. All the parameters are defined in Figure 3.4.

\begin{tabular}{||c|c|c|c|c|c|}
\hline $\begin{array}{c}\text { Day/ } \\
\text { flash/ } \\
\text { stroke }\end{array}$ & $\begin{array}{c}\mathrm{R} 0-1008 \\
\text { risetime } \\
(\mu \mathrm{sec}) \\
\pm 100 \mathrm{nsec}\end{array}$ & $\begin{array}{c}\mathrm{R} \\
\text { amplitude } \\
(\mathrm{kV} / \mathrm{m})\end{array}$ & $\begin{array}{c}\text { Time from } \\
\text { start of } \mathrm{R} \text { toscil- } \\
\text { valiey }(\mu \mathrm{s}) \\
\pm 120 \mathrm{nsec}\end{array}$ & $\begin{array}{l}\text { Impul- } \\
\text { vol- } \\
\text { tages } \\
\text { sive } \\
\text { vol- } \\
\text { tages }\end{array}$ \\
\hline $232 / 1 / 1$ & .5 & 1.4 & 3.3 & & $\mathrm{X}$ \\
\hline $232 / 1 / 2$ & .6 & .4 & 1.8 & $\mathrm{X}$ & \\
\hline $232 / 1 / 3$ & .6 & 1.4 & 3.1 & & $\mathrm{X}$ \\
\hline $232 / 1 / 4$ & .4 & 1.2 & 3.2 & & $\mathrm{X}$ \\
\hline $232 / 1 / 5$ & .7 & 1.0 & 3.3 & & $\mathrm{X}$ \\
\hline $232 / 1 / 6$ & .6 & .3 & 1.1 & $\mathrm{X}$ & \\
\hline $232 / 1 / 7$ & .5 & 1.5 & 3.0 & & $\mathrm{X}$ \\
\hline $240 / 1 / 1$ & 1.1 & .5 & no valley & $\mathrm{X}$ & \\
\hline $240 / 1 / 2$ & 1.4 & .7 & no valley & $\mathrm{X}$ & \\
\hline $240 / 2 / 1$ & 1.2 & .5 & 1.32 & $\mathrm{X}$ & \\
\hline $240 / 2 / 2$ & 1.3 & .6 & no valley & $\mathrm{X}$ & \\
\hline
\end{tabular}


Table 3.2. Some parameters of oscillatory voltages at the east end of the test line. All the parameters are defined in Figure 3.8a.

\begin{tabular}{|c|c|c|c|c|c|c|c|}
\hline $\begin{array}{l}\text { Day/ } \\
\text { flash/ } \\
\text { stroke }\end{array}$ & $\begin{array}{c}U_{0} \\
\text { ampli- } \\
\text { tude } \\
\text { (kV) }\end{array}$ & $\begin{array}{c}\mathrm{T}_{0} \\
\text { ampli- } \\
\text { tude } \\
(\mathrm{kV})\end{array}$ & $\begin{array}{l}\text { Subsid- } \\
\text { iary } 1 \\
\text { Pulse } \\
\text { ampli- } \\
\text { tude } \\
\text { (kV) }\end{array}$ & $\begin{array}{c}\text { Subsid- } \\
\text { iary } 2 \\
\text { Pulse } \\
\text { ampli- } \\
\text { tude } \\
\text { (kV) }\end{array}$ & 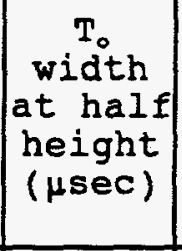 & $\begin{array}{l}\text { Time } T_{0} \\
\text { subsid- } \\
\text { iary } 1 \\
(\mu s e c)\end{array}$ & $\begin{array}{c}\text { Time } \\
\text { subsid- } \\
\text { iary } 1 \\
\text { subsid- } \\
\text { iary } 2 \\
\text { ( } \mu \text { sec) }\end{array}$ \\
\hline $232 / 1 / 2$ & 13 & -26 & -41 & -33 & .7 & 3.5 & 3.3 \\
\hline $232 / 1 / 6$ & 12 & -36 & -53 & -36 & .9 & 3.6 & 3.1 \\
\hline $240 / 1 / 1$ & 24 & -48 & -31 & -21 & .7 & 3.4 & 3.1 \\
\hline $240 / 1 / 2$ & 8 & -90 & -56 & -31 & .5 & 3.3 & 3.2 \\
\hline $240 / 2 / 1$ & 27 & -33 & -31 & -26 & .8 & 2.9 & 3.3 \\
\hline $240 / 2 / 2$ & 31 & -45 & -43 & -26 & .9 & 3.1 & 2.8 \\
\hline
\end{tabular}

Table 3.3. Some parameters of oscillatory voltages at the west end of the test line. All the parameters are defined in Figure $3.8 \mathrm{a}$.

\begin{tabular}{|c|c|c|c|c|c|c|c|}
\hline $\begin{array}{l}\text { Day/ } \\
\text { flash/ } \\
\text { stroke }\end{array}$ & $\begin{array}{c}\mathrm{N}_{\mathrm{o}} \\
\text { ampli- } \\
\text { tude } \\
(\mathrm{kV})\end{array}$ & $\begin{array}{c}M_{\circ} \\
\text { ampli- } \\
\text { tude } \\
(\mathrm{kV})\end{array}$ & $\begin{array}{l}\text { Subsid- } \\
\text { iary } 1 \\
\text { Pulse } \\
\text { ampli- } \\
\text { tude } \\
\text { (kV) } \\
\end{array}$ & $\begin{array}{c}\text { Subsid- } \\
\text { iary } 2 \\
\text { Pulse } \\
\text { ampli- } \\
\text { tude (kv) }\end{array}$ & $\mid \begin{array}{c}M_{0} \\
\text { width } \\
\text { at half } \\
\text { height } \\
\text { (nsec) }\end{array}$ & $\begin{array}{c}\text { Time } M_{0} \\
\text { subsid- } \\
\text { iary } 1 \\
\text { (Hsec) }\end{array}$ & $\begin{array}{c}\text { Time } \\
\text { subsid- } \\
\text { iary 1 } \\
\text { subsid- } \\
\text { iary 2 } \\
\text { (Hsec) }\end{array}$ \\
\hline $232 / 1 / 2$ & 32 & -111 & -64 & -34 & 453 & 3.3 & 3.3 \\
\hline $232 / 1 / 6$ & 17 & -68 & -56 & -40 & 984 & 3.5 & 3.2 \\
\hline $240 / 1 / 1$ & 26 & -60 & -56 & -37 & 1,750 & 3.1 & 3.3 \\
\hline $240 / 1 / 2$ & 25 & -79 & -56 & -43 & 1,250 & 3.2 & 3.4 \\
\hline $240 / 2 / 1$ & 29 & -52 & -34 & $\ldots *$ & 806 & 3.0 & $---^{*}$ \\
\hline $240 / 2 / 2$ & 31 & -60 & -46 & $--{ }^{*}$ & 1,370 & 3.5 & $---^{*}$ \\
\hline
\end{tabular}


Table 3.4. Some parameters of impulsive voltages at the west end of the test line. All the parameters are defined in Figure $3.8 \mathrm{~b}$.

\begin{tabular}{||c|c|c|c|c|c|c||}
\hline $\begin{array}{c}\text { Day/ } \\
\text { flash/ } \\
\text { stroke }\end{array}$ & $\begin{array}{c}\mathrm{N}_{i} \\
\text { ampli- } \\
\text { tude } \\
(\mathrm{kV})\end{array}$ & $\begin{array}{c}\mathrm{M}_{i} \\
\text { ampli- } \\
\text { tude } \\
(\mathrm{kV})\end{array}$ & $\begin{array}{c}\text { Subsid- } \\
\text { iary neg- } \\
\text { Pulse } \\
\text { ampli-tude } \\
(\mathrm{kV})\end{array}$ & $\begin{array}{c}\text { Subsid- } \\
\text { iary pos. } \\
\text { Pulse } \\
\text { ampli-tude } \\
(\mathrm{kV})\end{array}$ & $\begin{array}{c}\mathrm{N}_{i} \text { width } \\
\text { at half } \\
\text { height } \\
(\mu s e c)\end{array}$ & $\begin{array}{c}\mathrm{M}_{i} \text { width } \\
\text { at half } \\
\text { height } \\
(\mu \mathrm{sec})\end{array}$ \\
\hline $232 / 1 / 1$ & 359 & -977 & -164 & 154 & 2.2 & .5 \\
\hline $232 / 1 / 3$ & 360 & -778 & -130 & 119 & 2.5 & .4 \\
\hline $232 / 1 / 4$ & 403 & $-1,000$ & -210 & 183 & 2.7 & .6 \\
\hline $232 / 1 / 5$ & 317 & -763 & -113 & 67 & .5 & .4 \\
\hline $232 / 1 / 7$ & 204 & -823 & -129 & 116 & 3.3 & .4 \\
\hline
\end{tabular}

Table 3.5. Some parameters of impulsive voltages at the east end of the test line. All the parameters are defined in Figure $3.8 \mathrm{~b}$.

\begin{tabular}{||c|c|c|c|c|c||}
\hline $\begin{array}{c}\text { Day/ } \\
\text { flash/ } \\
\text { stroke }\end{array}$ & $\begin{array}{c}\mathrm{U}_{i} \\
\text { ampli- } \\
\text { tude } \\
(\mathrm{kV})\end{array}$ & $\begin{array}{c}\mathrm{T}_{i} \\
\text { ampli- } \\
\text { tude } \\
(\mathrm{kV})\end{array}$ & $\begin{array}{c}\text { Subsid- } \\
\text { iary } \\
\text { Pulse } \\
\text { ampli-tudg } \\
(\mathrm{kV})\end{array}$ & $\begin{array}{c}\mathrm{U}_{i} \text { width } \\
\text { at half } \\
\text { height } \\
(\mu \mathrm{sec})\end{array}$ & $\begin{array}{c}\mathrm{T}_{\mathrm{i}} \text { width } \\
\text { at half } \\
\text { height } \\
(\mu \mathrm{sec})\end{array}$ \\
\hline $232 / 1 / 1$ & 114 & -350 & 93 & 1.6 & .7 \\
\hline $232 / 1 / 3$ & 105 & -380 & 79 & 1.7 & .8 \\
\hline $232 / 1 / 4$ & 116 & -397 & 97 & 1.6 & 1.0 \\
\hline $232 / 1 / 5$ & 59 & -272 & 41 & 1.5 & .6 \\
\hline $232 / 1 / 7$ & 96 & -370 & 67 & 2.0 & .8 \\
\hline
\end{tabular}

\subsubsection{Oscillatory Voltages}

In the following characterization, all amplitudes are measured with respect to zero level, where zero level is marked by the initial horizontal segment of the waveform. The oscillatory voltages measured at the end nearer to the triggering site (east end) start with a positive pulse whose front rises to peak in some $20 \mu \mathrm{sec}$. This pulse, which we term $U_{0}$, has a mean amplitude of $19 \mathrm{kV}$ with a standard deviation $9 \mathrm{kV}$. After this initial positive-going pulse a series of negative pulses ensues. We call the first three of these pulses $T_{0}$, subsidiary 1 , and 
subsidiary 2, respectively. Typically these pulses have monotonically decaying amplitudes although for the oscillatory voltages recorded on day 232 the second negative pulse is larger than the first. The mean amplitudes of the $T_{0}$, subsidiary 1 , and subsidiary 2 pulses are $-47,-43$, and $-29 \mathrm{kV}$, respectively, with corresponding standard deviations 21,10 and $5 \mathrm{kV}$. The mean width at half amplitude maximum of $T_{0}$ is $744 \mathrm{nsec}$ (standard deviation $149 \mathrm{nsec}$ ). The average time between the peaks of the $T_{0}$ and the subsidiary 1 pulses is $3.3 \mu \mathrm{s}$ (standard deviation $0.3 \mu \mathrm{s}$ ) whereas between the peak of the two subsidiary pulses this time is 3.1 Hs (standard deviation $0.2 \mu \mathrm{s}$ ).

The oscillatory voltages measured at the end farther from the triggering site (west end) exhibit features very similar to those described for the voltages measured at the near end: a slow positive pulse followed by a series of sharper negative pulses. We have labeled the positive pulse $N_{0}$ and the first negative pulse $M_{0}$. As in the case of the oscillatory voltages at the east end, we call the two next pulses subsidiary 1 and subsidiary 2, respectively. At the west end, the voltages present monotonically decaying pulses in all the events recorded. The mean peak amplitude of $\mathrm{N}_{0}$ is 27 kVolts (standard deviation 5 kvolts). The mean peak amplitudes of the $M_{0}$, subsidiary 1 , and subsidiary 2 pulses are $-72,-53$, and -39 kVolts, respectively, with corresponding standard deviations of 20,10 , and 3 kVolts. The mean width at half amplitude maximum of $M_{0}$ is $1.1 \mathrm{\mu sec}$ with standard deviation 0.4

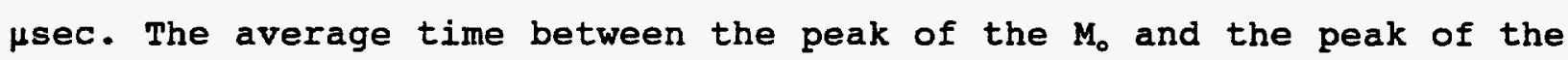
subsidiary 1 pulses is $3.3 \mu s$ (standard deviation $0.2 \mu s$ ) and between the peaks of the subsidiary 1 and the subsidiary 2 pulses this time is also 3.3 Hs (standard deviation $0.1 \mu s$ ). 
The main difference observed between typical voltages of the oscillatory type measured at the east and at the west ends of the line is that the west end voltage is larger and the east end voltage has a higher negative offset on the voltage reflections.

\subsubsection{Impulsive Voltages}

Impulsive voltages at the end nearer to the rocket triggering site (east end) are now characterized. Recall that all amplitudes are defined with respect to the initial waveform level. The waveforms start with a slow positive pulse exhibiting about a $20 \mu \mathrm{sec}$ duration and ending in finer structure, typically characterized by two positive-going peaks. The overall initial positive pulse is termed $U_{i}$. It has an average peak amplitude of 98 kVolts (standard deviation 21 kVolts). The mean width at half height of this pulse is $1.7 \mu \mathrm{sec}$ (standard deviation $0.17 \mu \mathrm{sec}$ ). A large negative pulse, which we have labeled $T_{i}$, succeeds the positive pulse $U_{i}$. This pulse has a mean peak amplitude of -354 kVolts and a standard deviation of 44 kVolts. The mean width of $T_{i}$ is 783 nsec with standard deviation $123 \mathrm{nsec}$. After this pulse has dropped to zero, or near zero, a smaller positive-going pulse occurs with mean peak amplitude 76 kVolts (standard deviation 20 kVolts), followed by a slow upward hump of some $25 \mu \mathrm{sec}$ width.

We now characterize the impulsive voltages observed at the end farther from the triggering site (west end). These voltage waveforms start with a positive pulse which exhibits some structure much like the start of the impulsive voltages at the near end. We call this pulse $\mathrm{N}_{i}$. The width at half amplitude maximum of this pulse has a mean value of 2.2 $\mu$ sec and a standard deviation of $0.9 \mu \mathrm{sec}$. The end of this pulse is marked by a sharp positive peak. The peak amplitude of this pulse has a mean of 329 kVolts with standard deviation 68 kVolts. A large negative 
pulse which determines the maximum amplitude of the waveform follows with an average peak amplitude of $-870 \mathrm{kVolts}$ (standard deviation $102 \mathrm{kVolts}$ ). This large negative pulse, which we call $M_{i}$, exhibits a mean duration of $493 \mathrm{nsec}$ (standard deviation $86 \mathrm{nsec}$ ). After this pulse has dropped to near zero, it is followed by a small negative pulse and a small positive pulse. The mean peak amplitudes of the negative and positive pulses are 149 kVolts (standard deviation 35 kVolts) and 128 kVolts (standard deviation $39 \mathrm{kVolts}$ ), respectively. A slow hump change similar to that encountered in the east end ensues lasting about $20 \mu s e c$. A series of small pulses is superimposed on this slow change.

Although the wave shapes for the east-end and west-end impulsive voltages are similar, the waveforms differ in that (1) The west-end voltages have amplitudes that are about twice the amplitudes of the voltages measured at the east end of the line, (2) the west-end voltages exhibit two small pulses, one negative and one positive, after the main negative pulse, while the voltages at the east end of the line have only one small positive pulse, and (3) a series of oscillations, absent in the east-end voltages, is observed to follow the second subsidiary pulse in the west-end voltages.

\subsection{Recent Measurements of Vertical and Horizontal Electric Fields 30 Meters From Triggered Lightning}

Measurements of the channel-base current simultaneous with the vertical and horizontal components of the electric field $30 \mathrm{~m}$ from artificially initiated lightning were made at the Kennedy space Center during the summer of 1991. The horizontal electric field was measured at two different heights, $1.4 \mathrm{~m}$ and $0.5 \mathrm{~m}$, above sea level by means of two spherical sensors manufactured by Thomson CSF. A flat plate antenna was used to record the vertical electric field derivative at sea level and the field was derived by computer integration. One of the spherical 
sensors was also employed to measure the vertical electric field during one of the experiment days. A current shunt was used to sample the channel-base currents. The field and current information was relayed via fiberoptics cable to a four-channel 9424 Lecroy oscilloscope where the signals were digitized at a rate of 100 megasamples per second. Eleven lightning flashes with a combined total of 23 strokes were recorded. Although for none of the strokes are all four measurements properly recorded simultaneously, different combinations of up to three measurements were recorded for each stroke.

Figure 3.9 shows an example of a measured vertical electric field waveform. The overall vertical electric field waveforms are v-shaped, the leading edge being a negative change associated with the dart leader and the trailing edge being a positive return stroke change. The width of this "negative" pulse is of the order of two microseconds. The unexpectedly-fast leader fields are necessarily a major factor in the coupling of close lightning fields to overhead wires, a fact not previously appreciated.

Figure 3.10 shows an example of a horizontal field waveform measured about $1.5 \mathrm{~m}$ above sea-water level. The horizontal electric field exhibits a leader change which is not dissimilar to that of the vertical electric field; however, the leader is immediately followed by a U-shaped field change with a small pulse at its onset.

These data are preliminary and are still under analysis at the time this report is being written. 


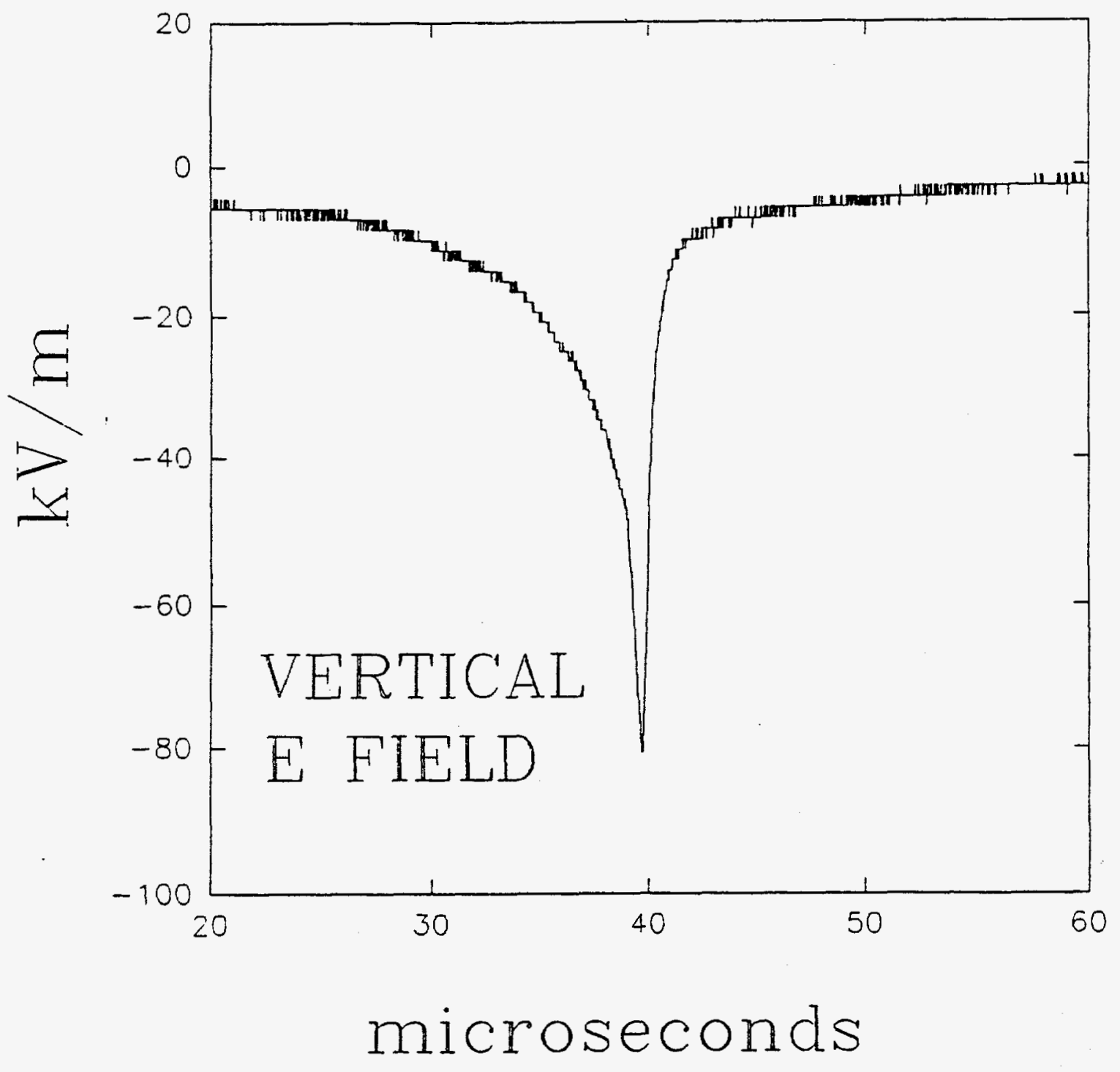

Fig. 3.9 Vertical electric field waveform measured $30 \mathrm{~m}$ from triggered lightning. 


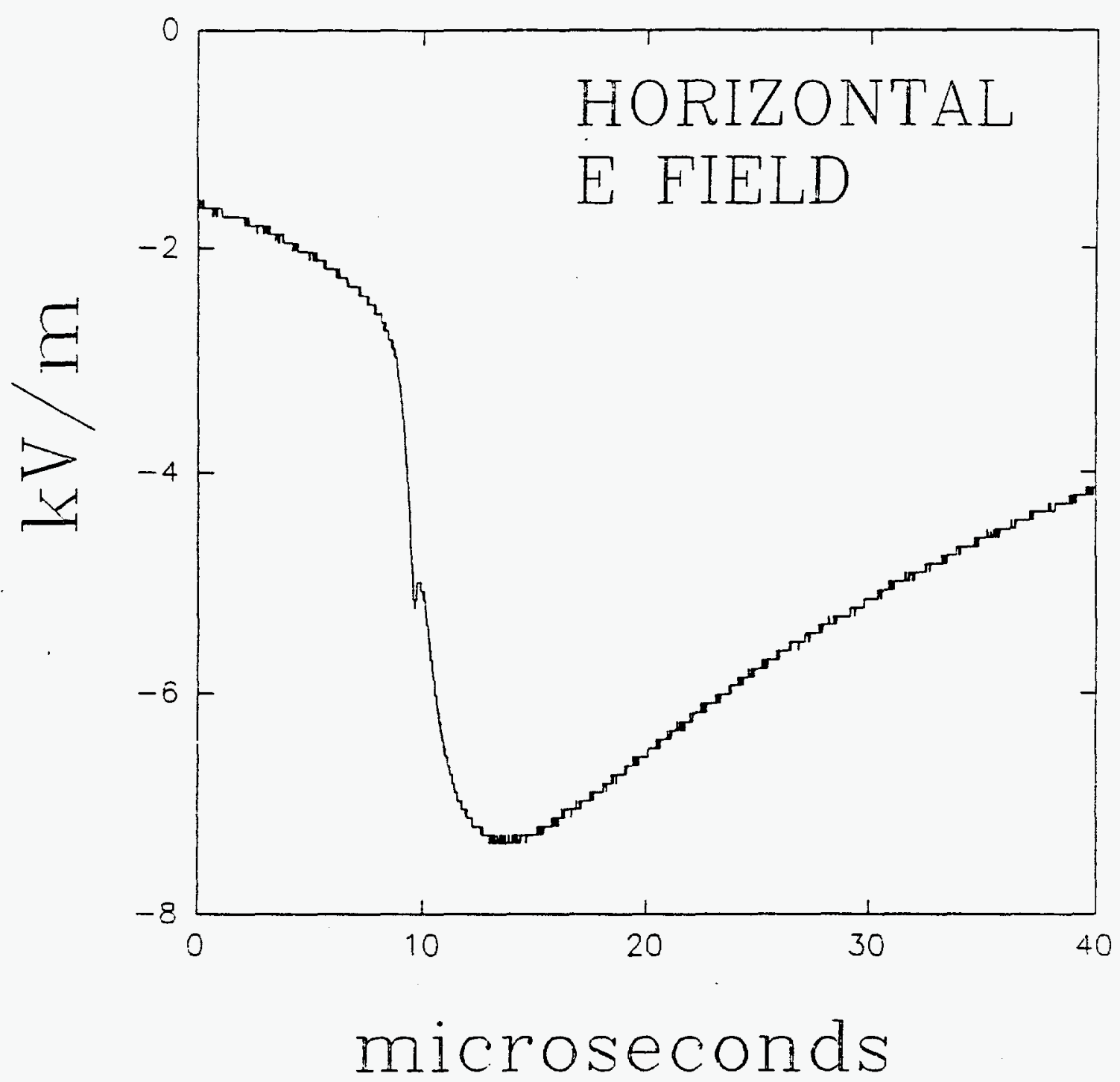

Fig. 3.10 A horizontal electric field waveform measured $30 \mathrm{~m}$ from triggered lightning at a height of $1.5 \mathrm{~m}$ above salt water. 
CALCULATION OF THE CAUSATIVE FIELDS FOR THE COUPIING OF CIOUD IIGHTNING FIELDS TO OVERHEAD POWER IINES

\subsection{Introduction}

In this chapter the relationship between the components of the electric field of an electromagnetic plane wave striking an overhead power line at an arbitrary direction is presented. This relationship is presented, for the case of grazing incidence, in section 6.2.1. The proof of the inadequacy of the application of the grazing incidence wavetilt formula used in previous studies of ground lightning (Master and Uman, 1984; Rubinstein et al., 1989) to the case of overhead lightning is presented. A new wavetilt formula for the non-grazing incidence case is developed.

\subsection{Incident Plane Wave With Arbitrary Polarization}

\subsubsection{Introduction}

In the presence of cloud lightning activity around an overhead power line, one can expect electromagnetic waves radiated by the different cloud-lightning processes to strike the line with arbitrary elevation and azimuth angles. Additionally, because the cloud lightning channel can have an arbitrary direction and can transport either negative or positive charge, one can expect the plane wave to strike the overhead power line with an arbitrary polarization angle. As noted earlier, we define the elevation angle, $\Phi$, as the angle above the horizon, and the azimuth angle, $\phi$, as the angle in the plane parallel to ground taken from the negative $x$ or westerly direction clockwise (CW) for an observer above the ground. Further, we define the polarization angle, $\alpha$, as the angle that the incident electric field, $\underline{E}^{i}$, makes with the semi-infinite horizontal line parallel to the plane of the ground and extending to the left of the 
direction of propagation as seen looking in that direction and perpendicular to it. Figure 4.1 shows the definition of the three angles.

The arbitrary polarized incident electric field, $\underline{E}^{i}$, can be divided into two components: parallel polarization component, $\underline{E}^{i}$, and the perpendicular polarization component, $\underline{E}^{i}$. These components are related to the $\underline{E}^{i}$ by the polarization angle, $\alpha$, as follows

$$
\begin{aligned}
& E_{1}^{1}=E^{1} \sin (\alpha) \\
& E_{1}^{1}=E^{1} \cos (\alpha)
\end{aligned}
$$

The purpose of the derivation that follows is to find the total vertical and horizontal components of the electric field at line height. Thus, the vertical and the horizontal components of the electric field for each polarization--parallel and perpendicular--will be obtained separately and then added together to get the total vertical and horizontal components. 4.2.2 Parallel Polarization

The parallel-polarized component of the incident electric field, $\underline{E}^{i}$, will have a vertical and a horizontal component as can be seen from Figure 4.2 .

With the help of Figure 4.1 and choosing the zero phase point at the west end of the line (i.e. $x=0$ and $z=h$ ), the complete phaser representation of $\underline{E}^{i}$ can be written as follows 


$$
\begin{aligned}
E_{1}^{i}= & E_{1}^{i}\left(\sin \Phi \cos \phi \hat{u}_{x}-\sin \Phi \sin \phi \hat{u}_{y}+\cos \Phi \hat{u}_{z}\right) \cdot \\
& e^{-j k_{0}[x \cos \Phi \cos \phi-y \cos \phi \sin \phi-(z-h) \sin \Phi]}
\end{aligned}
$$

Where $k_{0}=2 \pi / \lambda$ with $\lambda$ the wavelength and the 0 's are unit vectors in the $x, y$, and $z$ directions. Since it is irrelevant where the origin of the coordinate system is chosen, let the origin be such that the power line is in the $x-z$ plane, i.e., $y=0$. Then $E q \cdot(4.3)$ reduces to

$$
\begin{gathered}
E_{1}^{i}=E_{1}^{1}\left(\sin \Phi \cos \phi \hat{u}_{x}-\sin \Phi \sin \phi \hat{u}_{y}+\cos \Phi \hat{u}_{z}\right) \\
e^{-j k_{0}[x \cos \Phi \cos \phi-(z-h) \sin \Phi]}
\end{gathered}
$$

Moreover, to find the incident electric field at line height, the line height can be substituted for $z$ in the above equation

$$
\begin{gathered}
E_{1}^{i}(x, h)=E_{1}^{1}\left(\sin \Phi \cos \phi \hat{u}_{x}-\sin \Phi \sin \phi \hat{u}_{y}+\cos \Phi \hat{u}_{z}\right) \\
e^{-j k_{0} x \cos \Phi \cos \phi}
\end{gathered}
$$

However, the total electric field is the sum of both the incident and the reflected. If we call the total parallel-polarized electric field $\underline{E}_{1}$, then

$$
E_{1}=E_{1}^{d}+E_{1}^{x}
$$

After we allow for the fact that the reflected electric field at line height will have suffered a phase delay due to propagation of $2 k_{\circ} h s i n \Phi$, the reflected parallel-polarized electric field at line height, $\underline{E}^{x},(x, h)$, can be written 


$$
\begin{gathered}
E_{1}^{\alpha}=E_{1}^{I}\left(-\sin \Phi \cos \phi \hat{u}_{x}+\sin \Phi \sin \phi \hat{u}_{y}+\cos \Phi \hat{u}_{z}\right) \cdot \\
e^{-j k_{0}[x \cos \phi \cos \phi+2 h \sin \Phi]}
\end{gathered}
$$

and

$$
E_{1}^{Y}=\Gamma_{1}^{E} \cdot\left(E_{1}^{i}\right)
$$

where

$$
\Gamma_{1}^{E}=\frac{\epsilon_{x}(1+\sigma / j \omega \epsilon) \sin \Phi-\left[\epsilon_{x}(1+\sigma / j \omega \epsilon)-\cos ^{2} \Phi\right]^{1 / 2}}{\epsilon_{I}(1+\sigma / j \omega \epsilon) \sin \Phi+\left[\epsilon_{x}(1+\sigma / j \omega \epsilon)-\cos ^{2} \Phi\right)^{1 / 2}}
$$

where $\epsilon_{x}, \boldsymbol{\epsilon}$, and $\sigma$ are the relative permitivity, permitivity, and conductivity of the ground, respectively, and $\omega$ is the radial frequency. Now, the only components of the electric field that will be used in the calculation of the induced voltages at the two ends are the $x-$ (horizontal component along the power line) and the $z$ - (vertical component of the electric field) components. From Egs. (4.5), (4.6), (4.7), and (4.8) the total $z$ - and $x$ - parallel-polarized electric field components at line height can be written as

$$
\begin{gathered}
E_{\mid z}(x, h)=E_{\mid}^{i} e^{-j k_{0}(x \cos \Phi \cos \phi)}\left(1+\Gamma_{\mid}^{E} e^{-j k_{0} 2 h s i n \phi}\right) \cdot \cos \Phi \\
E_{\mid x}(x, h)=E_{\mid}^{i} e^{-j k_{0}(x \cos \Phi \cos \phi)}\left(1-\Gamma_{\mid}^{E} e^{\left.-j k_{0} 2 h \sin \Phi\right)} .\right. \\
\sin \Phi \cos \phi
\end{gathered}
$$




\section{SOURCE}

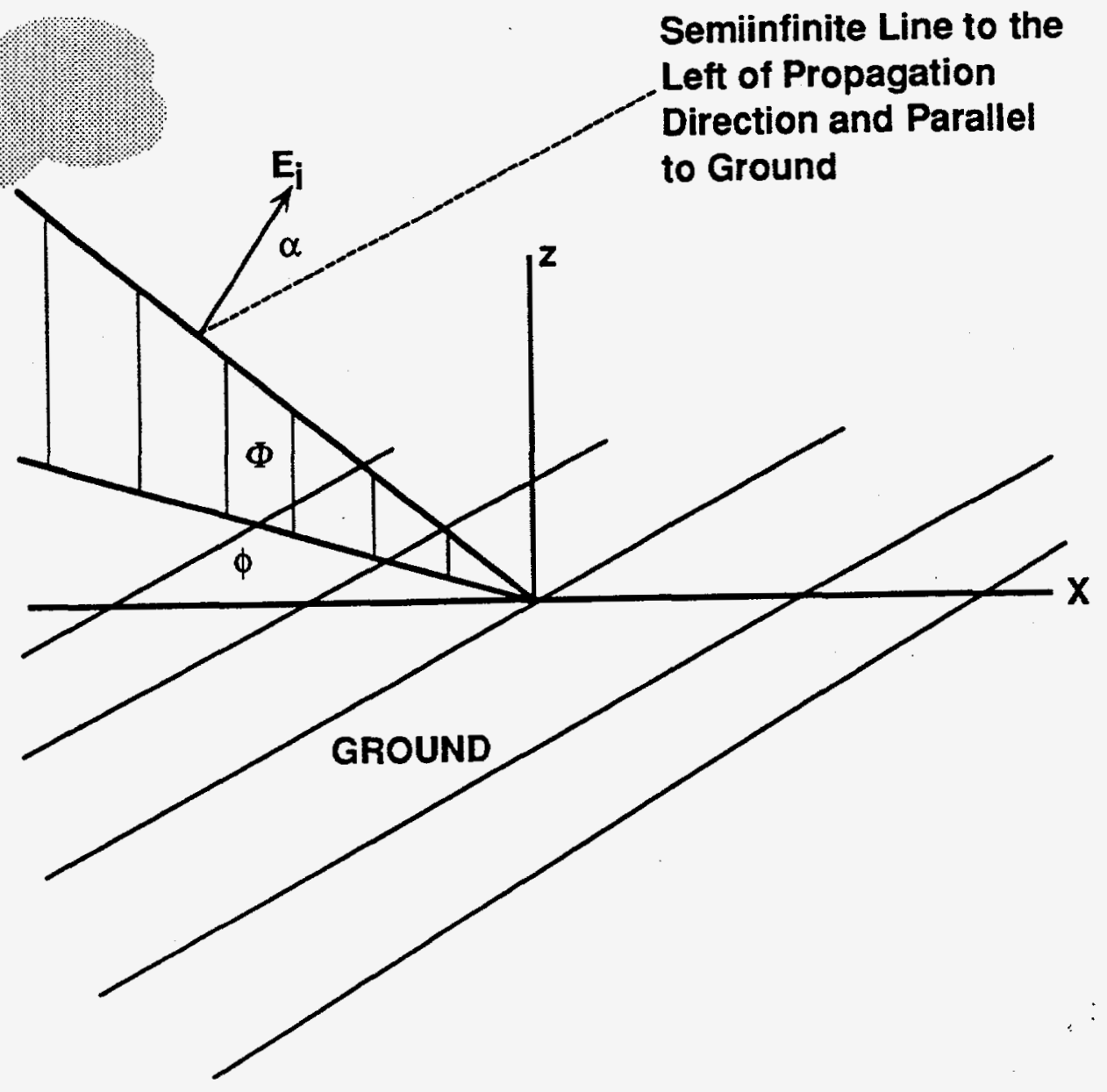

Fig. 4.1 Definition of the three direction angles 


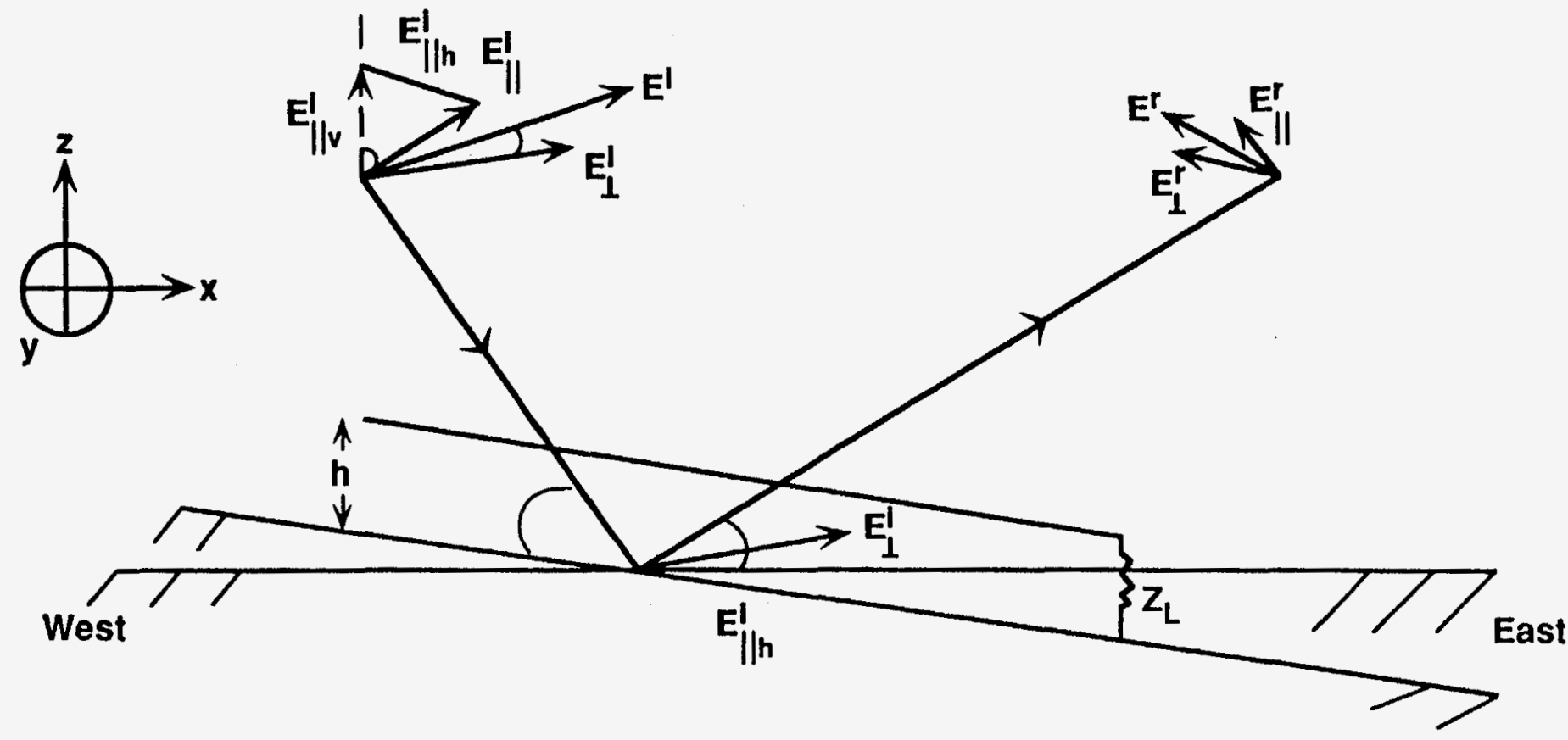

Fig. 4.2 The vertical and horizontal components of the parallelpolarized incident electric field. 


\subsubsection{Perpendicular Polarization}

With the help of Figure 4.1 , one can see that the perpendicularpolarized electric field only contributes a horizontal ( $x$ - and $y-$ ) component since $\underline{E}^{i}$ is completely in a plane parallel to the ground. Thus we can simply write the total vertical component of the perpendicularpolarized electric field

$$
E_{\perp_{z}}=0
$$

In a way similar to that used for parallel polarization, the total $x-$ component of the perpendicular-polarized electric field at line height can be written

$$
E_{\perp_{x}}(x, h)=E^{i} e^{-j k_{\rho} x \cos \Phi \cos \phi}\left(1+\Gamma^{E}{ }_{\perp} e^{-j k_{0} 2 h \sin \phi}\right) \cdot \sin \phi
$$

Where the perpendicular polarization reflection coefficient, $\Gamma^{\mathrm{E}}{ }_{1}$, is

$$
\Gamma_{\perp}^{E}=\frac{\sin \Phi-\left[\epsilon_{I}(1+\sigma / j \omega \epsilon)-\cos ^{2} \Phi\right]^{1 / 2}}{\sin \Phi+\left[\epsilon_{z}(1+\sigma / j \omega \epsilon)-\cos ^{2} \Phi\right]^{1 / 2}}
$$

Now, the total vertical $(z-)$ component, $E_{z}$, and along the line horizontal $(x-)$ component, $E_{x}$, at line height can be written using (4.1) and $(4.2)$ as follows

$$
E_{z}(x, h)=E^{1} e^{-j k_{\sigma} x \cos \phi \cos \phi}\left(1+\Gamma_{1}^{E} e^{-j k k_{\sigma} 2 h \sin \phi}\right) \cos \Phi \sin \alpha
$$




$$
\begin{aligned}
E_{x}(x, h)= & E^{1} e^{-j k x_{\sigma} \cos \Phi \cos \phi}\left[1-\Gamma^{E} e^{-j k_{\sigma} 2 h \sin \Phi}\right) \cdot \sin \Phi \cos \phi \sin \alpha \\
& \left.+\left(1+\Gamma^{E} e^{-j k_{\sigma} 2 h \sin \phi}\right) \cdot \sin \phi \cos \alpha\right]
\end{aligned}
$$

\subsection{Wavetilt for Non-Grazing Incidence}

\subsubsection{Introduction}

Rubinstein (1986), in his master's thesis, derived an expression for the non-grazing incidence wavetilt. However, the expression that he derived is only valid at ground since it was the ratio of the horizontal to vertical components of the electric field at ground level. Rubinstein also noted (private communication) that the magnitude of the horizontal component of the electric field, $E_{x}$, varies greatly with height, especially for elevation angles greater than $10^{\circ}$.

A computer program was written to verify these previous observations of Rubinstein. Figure 4.3 shows the variation of the magnitude of both the parallel-polarized and the perpendicular-polarized horizontal electric field components as a function of height and frequency. It is evident from Figure 4.3 that the horizontal component at $10 \mathrm{~m}$ can be more than 5 times that at ground. Therefore, a new wavetilt expression needed to be developed to involve the horizontal electric field component at line height and not at ground. Another computer program was written to show the variation of the magnitude of the vertical component of the electric field, $E_{z}$, with height as a function of elevation angle and frequency. The results are shown in Figure 4.4. It is also evident from this graph that, for frequencies below $1 \mathrm{MHz}$, the magnitude of the vertical component at $10 \mathrm{~m}$ is almost equal to that at ground. Thus, for our application, little error (less than 10 percent) is introduced in assuming that the vertical electric field does not vary with height. 

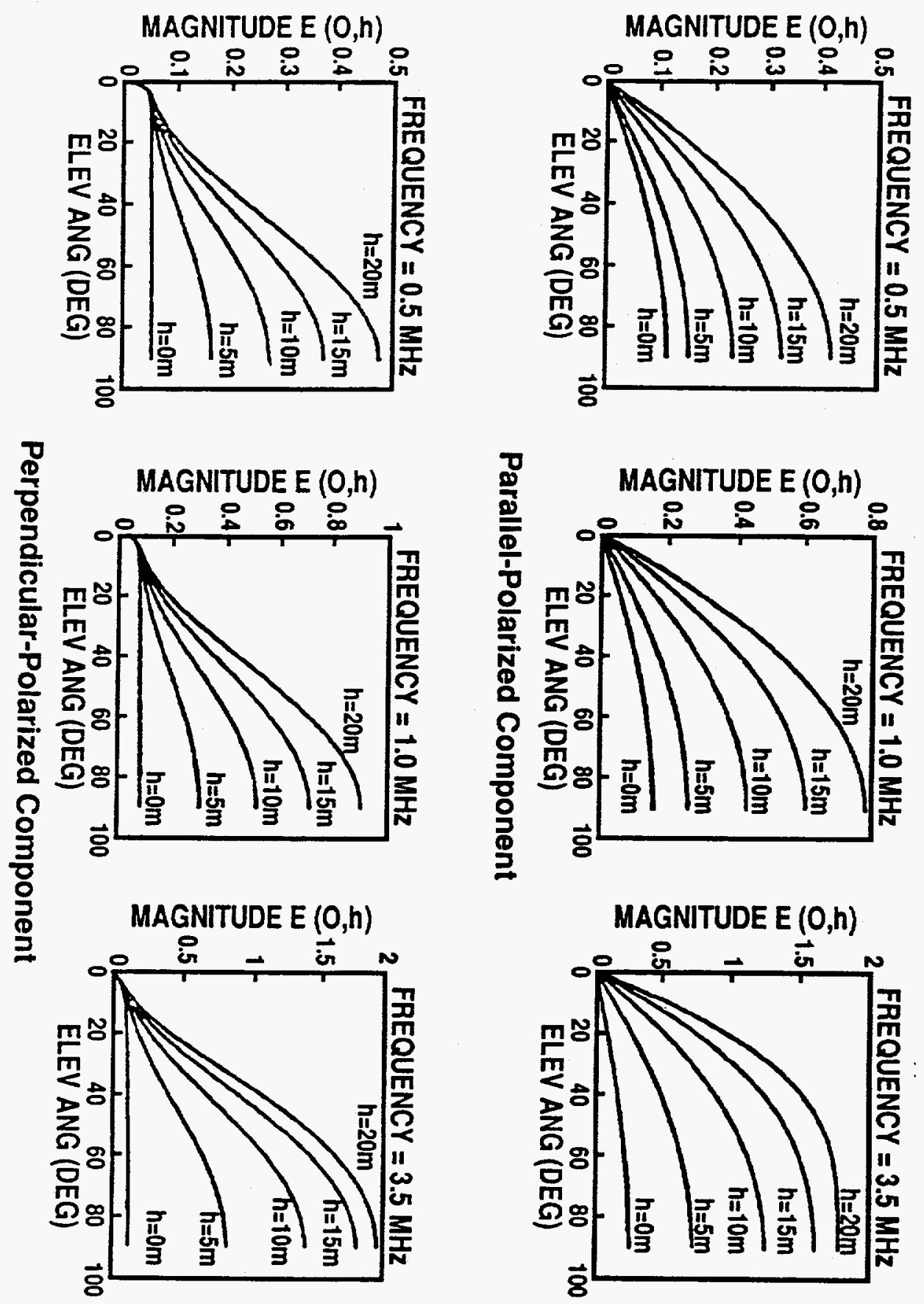

Fig. 4.3 Variation of the magnitude of both the parallel- and perpendicular-polarized horizontal electric field components with height, elevation angle, and frequency. 

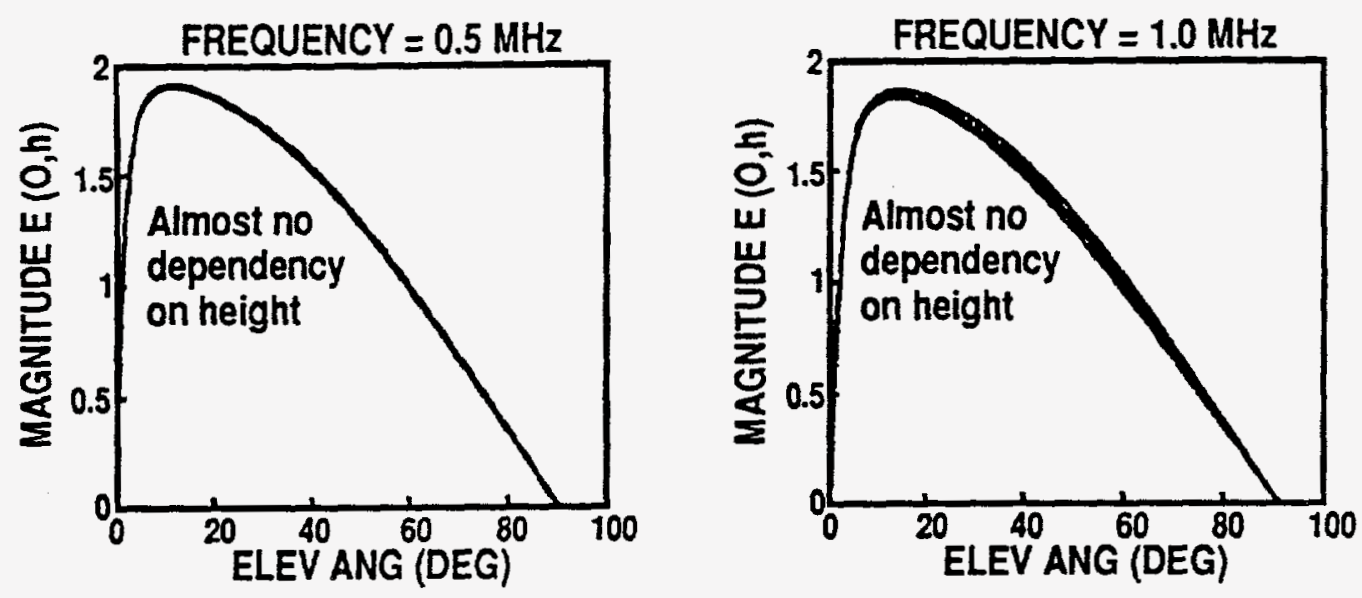

Vertical Electric Field
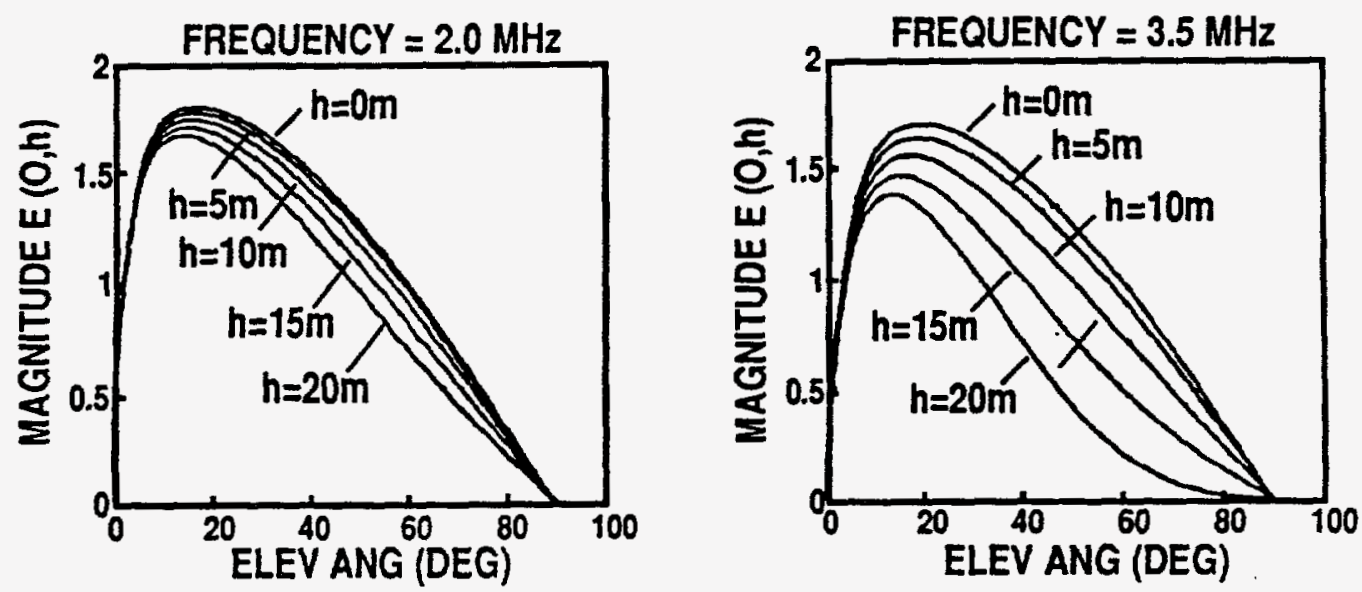

Fig. 4.4 variation of the magnitude of vertical component of the electric field with height, elevation angle, and frequency. 


\subsubsection{Derivation of Wavetilt Expressions}

Two wavetilt expressions will be derived in this section. The first wavetilt expression will be the ratio of the horizontal component along the line of the parallel-polarized electric field, $E_{\mid x}$, to the vertical component of the electric field, $E_{2}$, at line height. The second wavetilt expression will be the ratio of the horizontal component along the line of the perpendicular-polarized electric field, $E_{\perp x}$, to the vertical component of the electric field, $E_{z}$, at line height. Although the vertical electric field at ground is what is usually measured, the vertical electric field at line height is used in the wavetilt derivation because, as we have shown in Section 4.3 .1 , the vertical component of the electric field varies very little with height at the frequencies of interest.

Parallel polarization. The total electric field components at line height along and vertical to the power line are denoted as $E_{\mid x}(x, h)$ and $E_{z}(x, h)$. Then, the wavetilt ratio is defined as

$$
W=\frac{E_{\mid x}(x, h)}{E_{z}(x, h)}
$$

Now, using Eqs. (4.10) and (4.11) Eq. (4.17) can be written as

$$
W=\frac{\left(1-\Gamma_{1}^{E} e^{-j k_{o} 2 h \sin \Phi}\right) \sin \Phi \cos \phi}{\left(1+\Gamma_{1}^{E} e^{-j k_{o} 1 h \sin \Phi}\right) \cos \Phi}
$$

Using Eq. (4.9) and an approximation that $\varepsilon_{x}+\sigma / j \omega \varepsilon_{0}$ is much larger than unity, we can reduce Eq. $(4.18)$ to 


$$
W=A \cdot \frac{\left.j\left(\varepsilon_{x}+\sigma / j \omega \epsilon_{o}\right) \sin k_{o} h \sin \Phi\right) \sin \Phi+\left(\epsilon_{x}+\sigma / j \omega \epsilon_{0}\right)^{1 / 2} \cos \left(k_{o} h \sin \Phi\right)}{\left(\varepsilon_{z}+\sigma / j \omega \epsilon_{0}\right) \cos \left(k_{0} h \sin \Phi\right) \sin \Phi+j\left(\epsilon_{z}+\sigma / j \omega \epsilon_{0}\right)^{1 / 2} \sin \left(k_{o} h \sin \Phi\right)}
$$

where

$$
A=\tan \Phi \cos \phi
$$

As a check to this derivation, zero was substituted for $\mathrm{h}$ and Eq. (4.19) reduced to the wavetilt of elevated sources at ground that was derived by Rubinstein (1986)

$$
W=\frac{\cos \phi}{\cos \Phi \cdot\left[\epsilon_{x}+\sigma / j \omega \varepsilon_{0}\right]^{1 / 2}}
$$

Note that Eg. (4.19) has a cosф term in the numerator where as the wavetilt of Rubinstein (1986) does not. This is because the wavetilt that is derived here uses the horizontal electric field component along the line whereas Rubinstein (1986) uses the total horizontal electric field component.

Perpendicular polarization. In the same manner that the parallel polarization wavetilt was derived and using the same approximation, the wavetilt for perpendicular polarization can be derived using Eqs. (4.2), (4.12), (4.13), and (4.14). The following is the expression of the wavetilt

$$
W \sim B \cdot \frac{\left[\sin ^{2} \Phi \cos (K)+2 j \sin (K)\right]+\sin \Phi\left[2 C^{1 / 4} j \sin (K)+C^{-1 / 2} \cos (K)\right]}{C^{1 / 2} \sin \Phi \cos (K)+2 j \sin (K)}
$$

where 
$B=\frac{\tan \alpha \sin \phi}{\cos \Phi}$

$$
\begin{aligned}
& c=\left(\varepsilon_{x}+\sigma / j \omega \epsilon_{0}\right) \\
& R=2 k_{0} h \sin \Phi
\end{aligned}
$$

Again, if we were to substitute zero for h in Eq. (4.21), the same expression of the ground perpendicular polarization wavetilt for elevated sources will be obtained

$$
W \cdot \frac{\cot \alpha \cdot \sin \phi}{\cos \Phi \cdot\left[\epsilon_{x}+\sigma / j \omega \varepsilon_{0}\right]^{1 / 2}} \cdot\left\{\sin \Phi+\frac{1}{\left[e_{x}+\sigma / j \omega \epsilon_{0}\right]^{1 / 2}}\right\}
$$

The sind term in the numerator of Eq. (4.22) is due to the fact that the horizontal component along the line of the perpendicular polarized electric field is used in the wavetilt derivation. 



\section{CHAPTER V}

\section{COUPIING THEORY: TRANSMISSION IINE EQUATIONS IN THE TIME DOMAIN}

Some of the theories previously proposed to model the coupling phenomenon are not sufficiently rigorous since they either start out by assuming quasi-static conditions (e.g., Aigner, 1935; Szpor, 1948) or are not derived from the complete Maxwell's equations but rather from an intuitive approach (e.g., Lundholm, 1957, Rusk, 1958). Other workers have advanced more rigorous approaches, for example, Taylor et al. (1965) and Agrawal et al. (1980). Agrawal et al.'s model, being in the time domain, is perhaps the most important to test. In fact, his model has been tested by Master and Uman (1984), by Rubinstein et al. (1989), and by Georgiadis et al. (1992). In this section we derive the transmission line equations using the same general approach used by Agrawal et al.(1980). Even though the derivation presented here attacks the problem of a multi-wire transmission line with a ground plane as a reference, the same case studied by Master and Uman (1984), it deviates from their approach as follows: At the outset of their derivation, Master and Uman (1984) assumed the ground to be a perfect conductor. Consequently, they equated to zero both the horizontal component of the incident field at ground level, which they defined as the field from the lightning plus the effect of the perfectly conducting ground plane in the absence of the overhead wire, and the scattered horizontal electric field at ground level, which they defined as due to the currents induced in the overhead wire plus the effect of the perfectly conducting ground plane. They then accounted for the finite ground conductivity by adding a resistance per unit length of the ground and by using the wavetilt formula to calculate the horizontal electric field which was applied as 
a series line source. In contrast, in our development, the ground impedance and the fields in the model arise directly from the derivation.

The generation of the transmission line equations begins with the integration of Faraday's equation around a loop of differential width $\Delta x$ along the line as shown in Figure 5.1 .

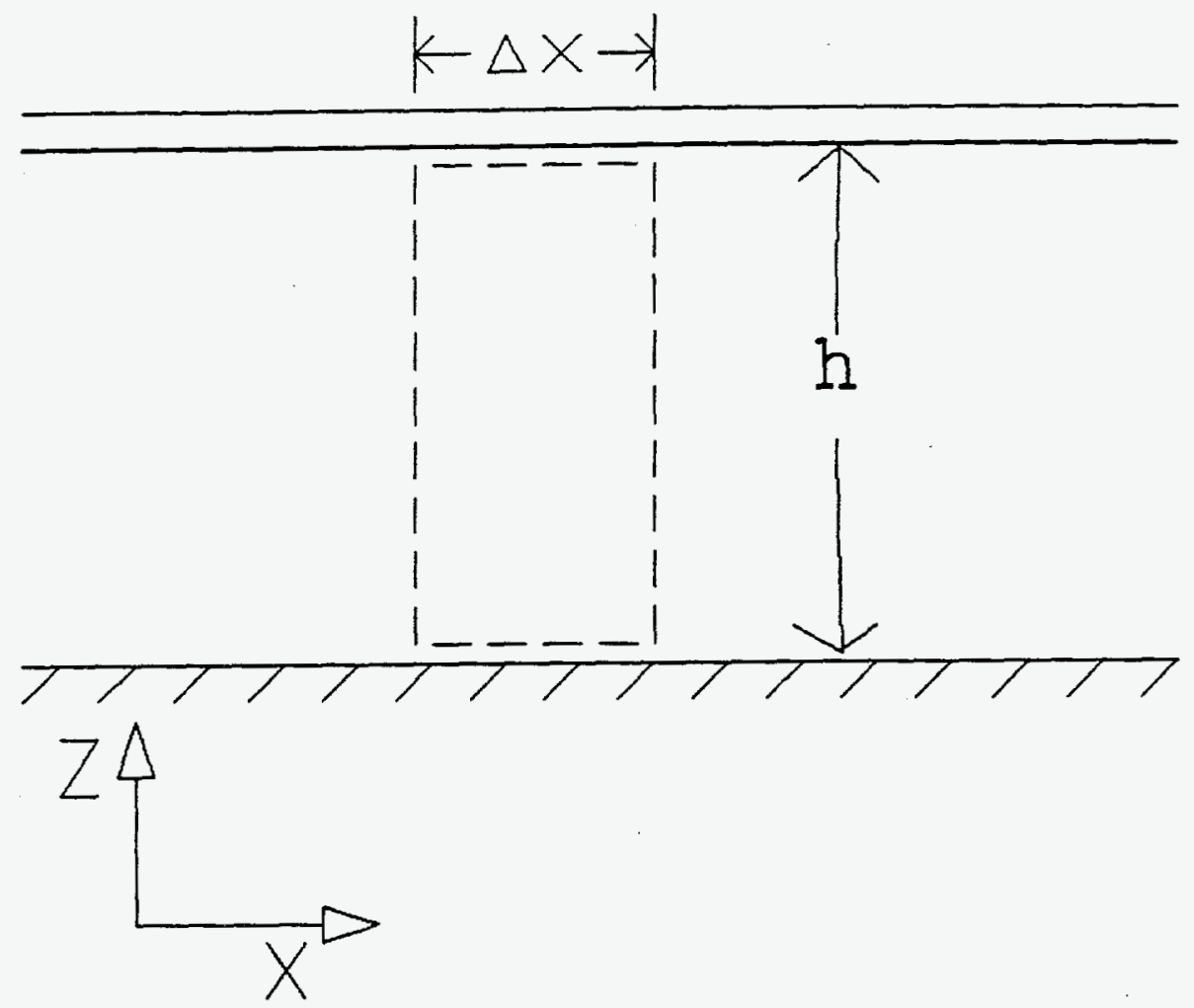

Fig. 5.1 Definition of the integration path in the derivation of the first Telegrapher's equation. The ground plane coincides with the $x-y$ plane and the overhead line is in the $x-z$ plane at $\mathrm{z}=\mathrm{h}$. 


$$
\begin{gathered}
\int_{0}^{h}\left[E_{z}(x+\Delta x, z)-E_{z}(x, z)\right] d z+\left[E_{x}(x, 0)-E_{x}(x, h)\right] \Delta x \\
=\Delta x \frac{\partial}{\partial t} \int_{0}^{h} B_{y}(x, z) d z
\end{gathered}
$$

Dividing Eq. (5.1) by $\Delta x$ and taking the limit as $\Delta x$ goes to 0 , we obtain

$$
\frac{\partial}{\partial x} \int_{0}^{h} E_{z}(x, z) d z+E_{x}(x, 0)-E_{x}(x, h)=\frac{\partial}{\partial t} \int_{0}^{h} B_{y}(x, z) d z
$$

The next step is to divide the fields in Eq. (5.2) into two components: the so-called incident fields and the so-called scattered fields. There exists some confusion as to what these two fields represent. The confusion stems from the fact that the incident field is sometimes taken to be the field that would exist if the transmission line, including the ground plane, were not present (i.e. Agrawal et al., 1980; Taylor, 1965); and other times as the field without the conducting wires but with the ground plane present (Master and Uman, 1984). Since the measured fields will unavoidably include the effect of the ground, we choose to use the latter definition, that is, we consider the incident field as the sum of the fields from the lightning current and the fields reflected from the ground, in the absence of the overhead wires. With this in mind we can write

$$
\begin{aligned}
& E_{z}=E_{z}^{1}+E_{z}^{s} \\
& E_{x}=E_{x}^{i}+E_{x}^{s} \\
& B_{y}=B_{y}^{1}+B_{y}^{s}
\end{aligned}
$$


The superscript $i$ denotes incident in the sense described above, and the superscript $s$ denotes scattered. The scattered fields are to be interpreted as being produced by the insertion of the overhead wires into the system. It is important to note that according to this definition the current sources responsible for the scattered fields are both the currents induced on the wires and the currents induced by them on the ground. These ground currents are different from and in addition to those associated with the incident fields. Substituting Eq. (5.3) into Eq. $(5.2)$, we get

$$
\begin{aligned}
\frac{\partial}{\partial x} \int_{0}^{h} E_{z}^{s}(x, z) d z & -\frac{\partial}{\partial t} \int_{0}^{h} B_{y}^{s}(x, z) d z+E_{x}^{s}(x, 0)-E_{x}^{s}(x, h) \\
=-E_{x}^{i}(z, 0) & +E_{x}^{i}(x, h)+\frac{\partial}{\partial t} \int_{0}^{h} B_{y}^{i}(x, z) d z \\
& -\frac{\partial}{\partial x} \int_{0}^{h} E_{z}^{i}(x, z) d z
\end{aligned}
$$

The incident fields must themselves satisfy Maxwell's equations. It follows that Eq. (5.2) can be written for the incident fields alone,

$$
\begin{gathered}
\frac{\partial}{\partial x} \int_{0}^{h} E_{z}^{i}(x, z) d z+E_{x}^{i}(x, 0)-E_{x}^{i}(x, h) \\
=\frac{\partial}{\partial t} \int_{0}^{h} B_{y}^{i}(x, z) d z
\end{gathered}
$$

Combining equations $(5.4)$ and $(5.5)$, we obtain 


$$
\begin{gathered}
\frac{\partial}{\partial x} \int_{0}^{b} E_{z}^{s}(x, z) d z+E_{x}^{s}(x, 0)-E_{x}^{s}(x, h) \\
=\frac{\partial}{\partial t} \int_{0}^{b} B_{y}^{s}(x, z) d z
\end{gathered}
$$

Our goal now is to write Eq. (5.6) in terms of voltages and currents on the line.

Let us make the following changes to Eq. (5.6):

1. Define the scattered voltage as follows

$$
V^{s}=-\int_{0}^{h} E_{z}^{s}(x, z) d z
$$

where the path of integration is a straight line extending vertically from the ground to the wire.

2. Add $E_{x}^{i}(x, h)$ to both sides of $E q \cdot(5.6)$, noting that $E_{x}^{i}(x, h)+E_{x}^{*}(x, h)$ $=E_{x}(x, h)$.

With these substitutions, we obtain

$$
\begin{gathered}
\frac{\partial}{\partial x} V^{s}(x, z)+E_{x}(x, h)-E_{x}^{s}(x, 0)+\frac{\partial}{\partial t} \int_{0}^{h} B_{y}^{s}(x, z) d z \\
=E_{x}^{i}(x, h)
\end{gathered}
$$

Up to this point, the derivation is exact. Normally (e.g., Agrawal et al., 1980), the horizontal fields are manipulated so that they can be approximated in the frequency domain as the product of a surface impedance per unit length, which for time domain calculations is assumed 
to be a real number, independent of frequency, times the current. We now investigate a more exact relation.

We first consider the relation between the total tangential electric field at the surface of each of the overhead wires and the total current flowing through it. By solving Maxwell's equations for propagation along a cylinder embedded in a dielectric medium, one can show that an infinite number of modes can be excited (Stratton, 1941). For a good conductor (e.g., copper or aluminum at frequencies from tens of Hertz to several Megahertz), only one of these modes, the axially symmetric or principal mode, can propagate with relatively low decay. The damping constant for this mode is of the order of hundreds of meters, as opposed to the several centimeter damping constants associated with the asymetrical modes. The fact that the diameter of the wires is small compared to (1) the wavelengths of interest, (2) the distance to the source, and (3) the distance between conductors, guarantees that the symmetrical mode will be exited whenever there is a component of the electric field in the axial direction. This is so because the wires can be treated as if they were immersed in a uniform field. Under these conditions, it can be proven that the current and the tangential field are related, in the frequency domain, by (Stratton, 1941)

$$
\hat{E}_{x}(x)=\left[R_{0}(1-j) a / 2 \delta\right] \hat{I}(x)
$$

where $R_{0}$ is the dc resistance per unit length of the wire, "a" is the radius of the conductor, $\delta$, the skin depth factor, is given by $1 /(\pi \mu \sigma f)^{1 / 2}$, and the circumflex signifies complex quantity. The factor multiplying $I$ is known as the surface impedance $z$. of the wire. In most practical cases, the surface impedance of the wire is much smaller than the surface impedance of the ground and can therefore be neglected if the two are in series. 
Approximations for the surface impedance of the ground have been obtained by Carson (1926), Vance (1978), Sunde (1968), and others. Of special importance is Vance's expression since it is probably the most commonly used in the context of electromagnetic coupling to overhead cables. This expression is given in Eq. (5.10) below,

$$
\begin{array}{ll}
\hat{z}_{g} \approx \frac{1+j}{2 \pi h \sigma \delta} & (\delta<\langle 2 h, \sigma\rangle>w E) \\
z_{g} \approx \frac{w \mu}{8}+j w \frac{\mu}{2 \pi} \log \frac{\sqrt{ } 2 \sigma}{\Gamma_{o} h} \quad(\delta>>2 h)
\end{array}
$$

Usually, Eq. (5.10a) is employed since, when typical electrical parameters are used $\left(\sigma=1.6 \times 10^{-2} \mathrm{mho} / \mathrm{m}\right.$, the measured conductivity at the Kennedy space Center experiment site, $\epsilon_{r}=10$, a typical value for the relative permittivity of wet earth, and $h=10$, the approximate height of the overhead line) the conditions $\sigma>>$ we and $\delta<<2 \mathrm{~h}$ imply that the frequency $f$ must satisfy the requirements $f<<30 \mathrm{MHz}$ and $f>350 \mathrm{kHz}$, respectively.

We will now derive a different expression for the "surface impedance of the ground" which we define in a different way from Vance (1978). We define the surface impedance as the ratio of the tangential electric field at ground level in the direction of the line and directly under the line divided by the line current. Apparently, Vance (1978) defines the surface impedance as the ratio of the tangential electric field at the line due to all effects, including the ground, to the line current, although this is not clear in his book. Let us first assume that we have a perfectly conducting ground plane. In this case the horizontal electric field at the surface of the ground is identically zero and there exists a horizontal magnetic field which can be calculated at every point on the surface of the ground by making use of image theory and magnetostatics. 
With the help of Figure 5.2, we can express the magnetic field at ground level as follows

$$
\hat{H}_{y}(z=0, y)=\frac{\hat{I} h}{\pi\left(y^{2}+h^{2}\right)}
$$

Let us assume now that the ground is no longer a perfect conductor but a "good" conductor instead. A small horizontal electric field will exist at ground level in the direction parallel to the overhead wire. The key assumption is that this field is a small perturbation and that the horizontal magnetic field is not appreciably affected by it. Integrating the Ampere-Maxwell equation around a rectangular path of width dy extending from $z=0$ to $z=-\infty$, we obtain

$$
\left[\hat{H}_{y}(y, z=0)-\int_{-\infty}^{0} \frac{\partial \hat{H}_{z}(y, z)}{\partial y} d z\right] d y \approx d I
$$

where we have neglected the displacement current in the conductor $(\sigma>>W E)$. We argue now that the integral on the left side of Eq. (5.12) is negligible with respect to the magnetic field in the $y$ direction. The approximation is justified as follows: assuming the fields in the ground propagate with a propagation constant equal to the skin $\operatorname{depth} \delta /(1+j)$ factor defined earlier in this chapter, Eq. (5.12) can be rewritten as follows

$$
\left[\hat{H}_{y}(y, z=0)-\frac{\partial \hat{H}_{z}(y, 0)}{\partial y} \frac{\delta}{(1+j)}\right] d y \approx d I
$$




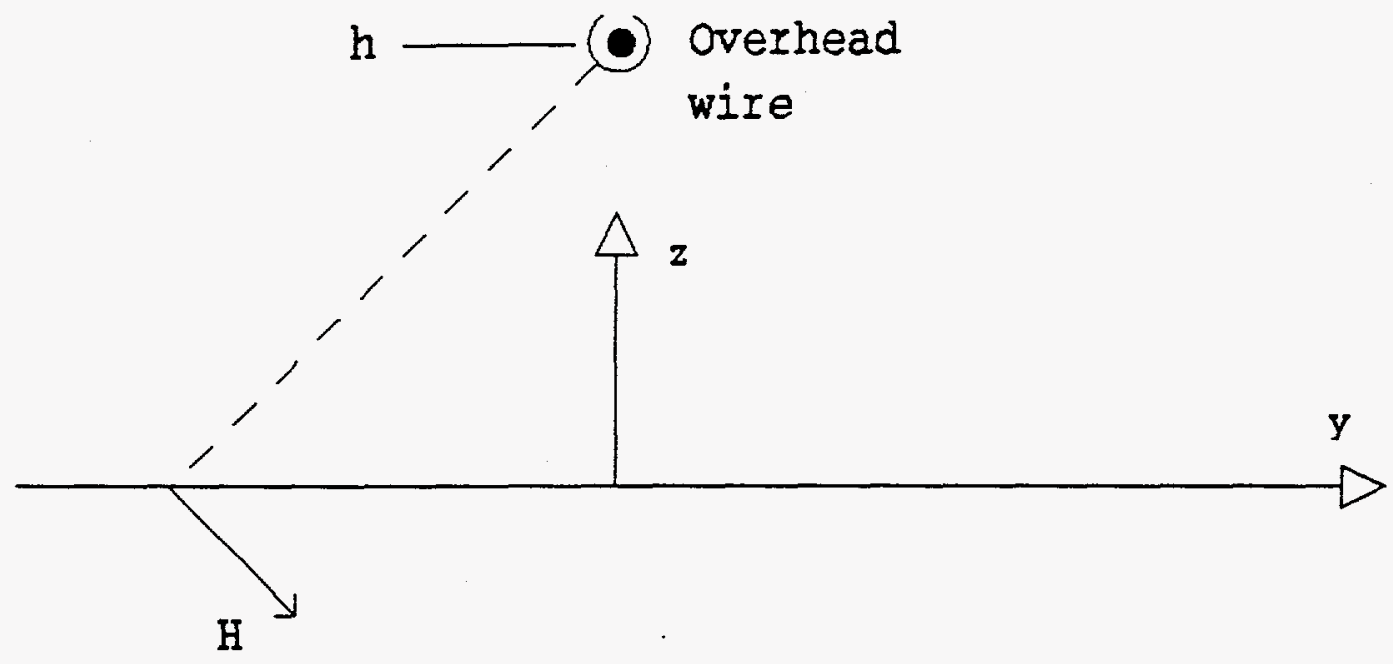

$\otimes$ Image

Fig. 5.2 Geometry for the calculation of the magnetic field from a current flowing through an overhead wire for the case of a perfectly conducting ground plane. 
The vertical component of the magnetic field at ground level is a consequence of the introduction of a finite ground conductivity and it can be crudely approximated (we are only interested in a rough estimate of its amplitude) as being due to the actual wire above the ground and an image located $2 \delta$ deeper than in the case of a perfectly conducting ground. This approximation is based on the assumption that the electric and magnetic fields are zero at a distance of one skin depth beneath ground level as shown in Figure 5.3. A more detailed calculation has been given by Gary (1976) who finds $2 \delta \rightarrow \frac{2 \delta}{1+j}$. From Figure 5.3 the field $\mathrm{H}_{2}(\mathrm{Y}, 0)$ can be found to be

$$
\hat{H}(y, 0) \approx \frac{\hat{I} y}{2 \pi}\left[\frac{1}{\left.y^{2}+(h+2 \delta)^{2}\right)}-\frac{1}{\left(y^{2}+h^{2}\right)}\right]
$$

and $\left.\partial \hat{H}_{z} / \partial y\right) \delta /(1+j)$ is

$$
\begin{gathered}
\frac{\delta}{(1+j)} \frac{\partial \hat{H}_{z}(y, 0)}{\partial y} \approx \frac{\hat{I} \delta}{2 \pi(1+j)}\left[\frac{1}{\left(y^{2}+(h+2 \delta)^{2}\right)}-\frac{1}{\left(y^{2}+h^{2}\right)} .\right. \\
-\frac{y^{2} \hat{I} \delta}{\pi(1+j)}\left[\frac{1}{\left(y^{2}+(h+2 \delta)^{2}\right)^{2}}-\frac{1}{\left(y^{2}+h^{2}\right)^{2}}\right]
\end{gathered}
$$

A plot of the ratio of the magnitude of Eq. (5.15) to Eq. (5.11) as a function of $y$ with the skin depth $\delta$ as a parameter is shown in Figure 5.4. This figure reveals that the integral is in fact negligible for $\delta<h$ with $h \approx 10 \mathrm{~m}$. Eq. (5.13) can now be rewritten as follows

$$
\hat{H}_{y}(y, z=0) d y \approx d I \quad(\delta\langle h, \sigma\rangle>w E)
$$

but

$$
\delta I=\frac{\sigma \hat{E}_{x}(y, z=0) \delta d y}{(1+j)}
$$




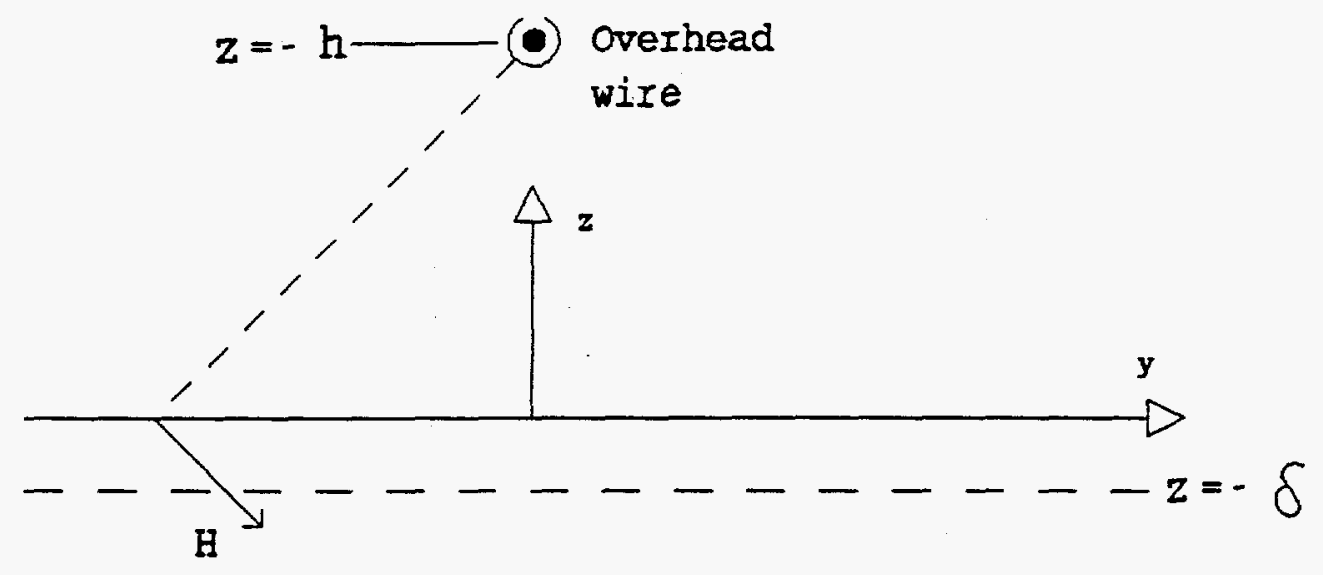

$z=-(h+2 \delta)-\otimes$ Image

Fig. 5.3 Geometry for the approximate calculation of the magnetic field from a current flowing through an overhead wire for the case of an imperfectly conducting ground plane. 


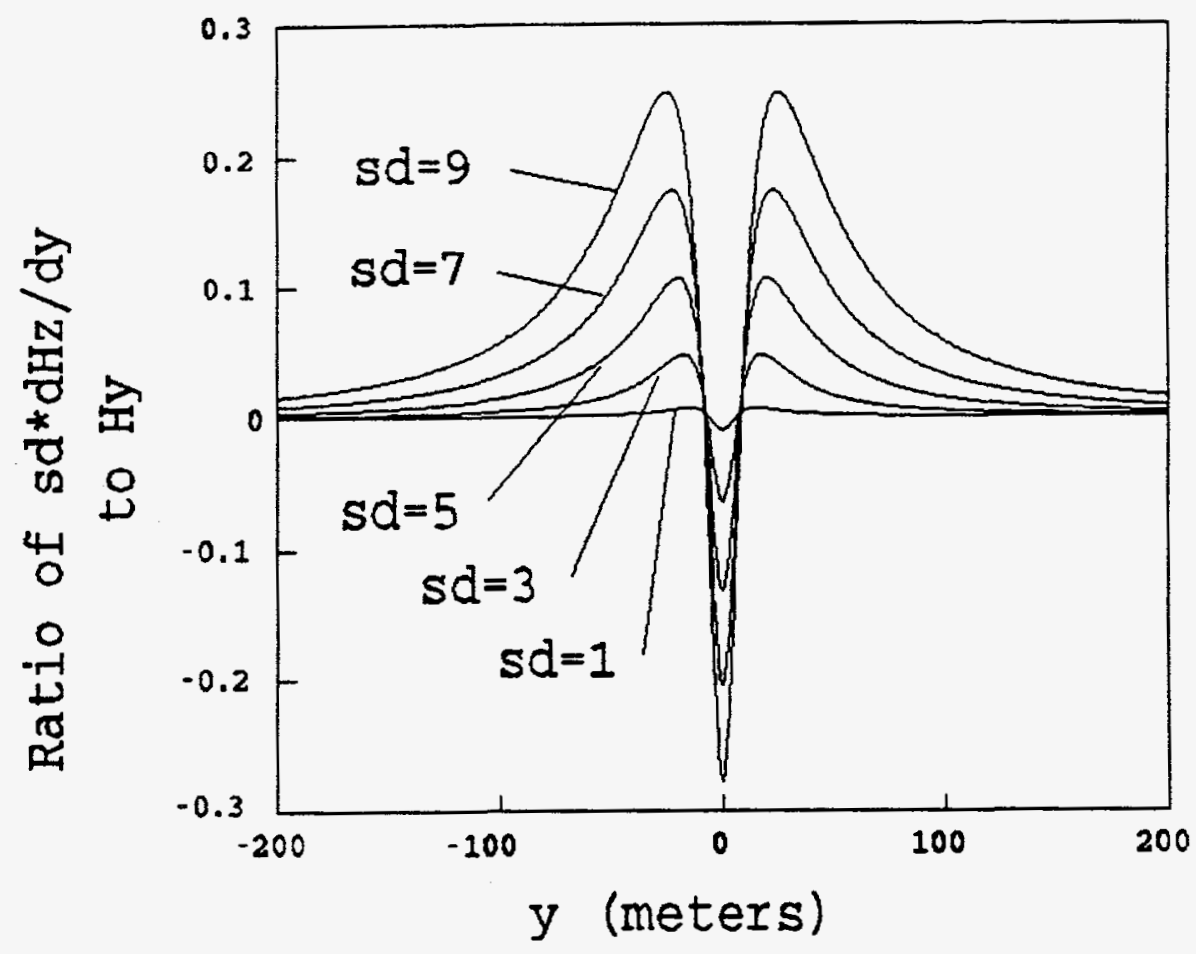

Fig. 5.4 Plots of the ratio of $\delta \partial H_{z}(y, 0) / \partial y$ to $H_{y}(z=0, y)$ as a function of $Y$. 
Combining Eqs. $(5.16)$ and (5.17), we get

$$
\hat{H}_{y}(y, z=0) d y \approx \frac{\sigma \hat{E}_{x}(y, z=0) \delta d y}{(1+j)} \quad(\delta\langle\langle h, \sigma\rangle\rangle w \epsilon)
$$

from which $\hat{E}_{x}(y=0, z=0)$ can be found to be

$$
\hat{E}_{x}(y=0, z=0) \approx \frac{(1+j)}{\pi h \sigma \delta} \hat{I}
$$

and the surface impedance is

$$
\hat{z}_{g} \approx \frac{(1+j)}{\pi h \sigma \delta}
$$

This expression is a factor of two greater than the expression given in Vance (1987) due to our different definition of the surface impedance. In the case of a perfectly conducting ground, the return current is identical to the current in the overhead wires but in the opposite direction. We will assume that this relationship is still valid in our case. If the current flowing in the positive $x$ direction in the overhead wire is $I_{w}$, the current in the ground, also defined in the positive $x$ direction, is $I_{g}(x)=-I_{w}(x)$. Hence, we can write the following equation in the frequency domain

$$
\hat{E}_{x}^{s}(x, 0, w)=-\hat{z}_{g} \hat{I}_{w}(x, w)
$$

or, in the time domain,

$$
E_{x}^{s}(x, 0)=-Z_{g} * I_{w}(x)
$$


where we have not used the circumflex (") over the variables to signify their time domain representations and where "*" is the convolution operator.

Only the magnetic flux term remains to be written in terms of measurable parameters in Eq. (5.8). This relation can be expressed as follows

$$
\frac{\partial}{\partial t} \int_{0}^{h} B_{y}^{s}(x, z) d z=L \frac{\partial I_{w}(x)}{\partial t}
$$

where $I$, the value of the inductance, is calculated by dividing the magnetic flux per unit length (given by the integral on the left-hand side of $\mathrm{Eg}$. (5.23) by the current in the wire $I_{w}(x)$. The implicit assumption here is that the current in the wire is proportional to the magnetic flux per unit length from $z=0$ to $z=h$. In reality, the effect of an imperfect ground is to make the inductance a weak function of frequency. We can justify this assertion by calculating the inductance for the case of a perfectly conducting earth and comparing it to the inductance for the case of an imperfect earth as a function of frequency. For a perfectly conducting earth and for the line height $h$ much greater than the radius of the overhead wire "a", the inductance $I$ is given by

$$
L=\frac{\mu_{0}}{2 \pi} \ln \left(\frac{2 h}{2}\right)
$$

where " $\mu_{0}$ " is the permeability of free space. The effect of an imperfect ground on the magnetic field can be roughly estimated by assuming that the tangential electric field is zero one skin depth beneath the ground as shown in Figure 5.3 .

Using this approximation, the total magnetic flux from the ground level to the height of the wire can be calculated using image theory as 
the superposition of the effects of the overhead wire and its image located symmetrically beneath the zero-tangential-electric-field level, two skin depths plus $h$ under the ground as shown in Figure 5.3. The flux density as a function of distance $\rho$ to the current (or the image) I is given by

$$
B=\frac{\mu_{0} I}{2 \pi \rho}
$$

The total flux for the imperfect ground case can therefore be approximated as

$$
\phi_{\text {totaI }}=\int_{a}^{h} \frac{\mu_{0} I}{2 \pi \rho} d \rho+\int_{h+2 \delta}^{2 h+2 \delta-a} \frac{\mu_{0} I}{2 \pi \rho} d \rho
$$

Evaluating the integrals in Eq. (5.26) and dividing the result by $I$, we obtain the following expression for the inductance

$$
I=\frac{\mu_{0}}{2 \pi} \ln \left(\frac{h}{a}\right)+\frac{\mu_{0}}{2 \pi} \ln \left(1+\frac{h-a}{h+2 \delta}\right)
$$

The percent error between the values of I obtained making use of eqs. (5.24) and (5.26) has been plotted as a function of frequency in Figure 5.5 , with the first point corresponding to $10 \mathrm{kHz}$. It is clear from this figure that the use of a perfectly conducting ground model for the derivation of the inductance is indeed justified. Now, substituting Eqs. (5.22) and (5.23) into Eq. (5.8), noting that the surface impedance relation given in Eq. (5.21) has been derived for the fields produced by the current in the wire, that is, the scattered fields, we obtain

$$
\begin{aligned}
\frac{\partial}{\partial x} V^{s}(x, z) & +Z_{g} * I_{w}(x)+L \frac{\partial I_{w}(x)}{\partial t} \\
= & E_{x}^{i}(x, h)
\end{aligned}
$$




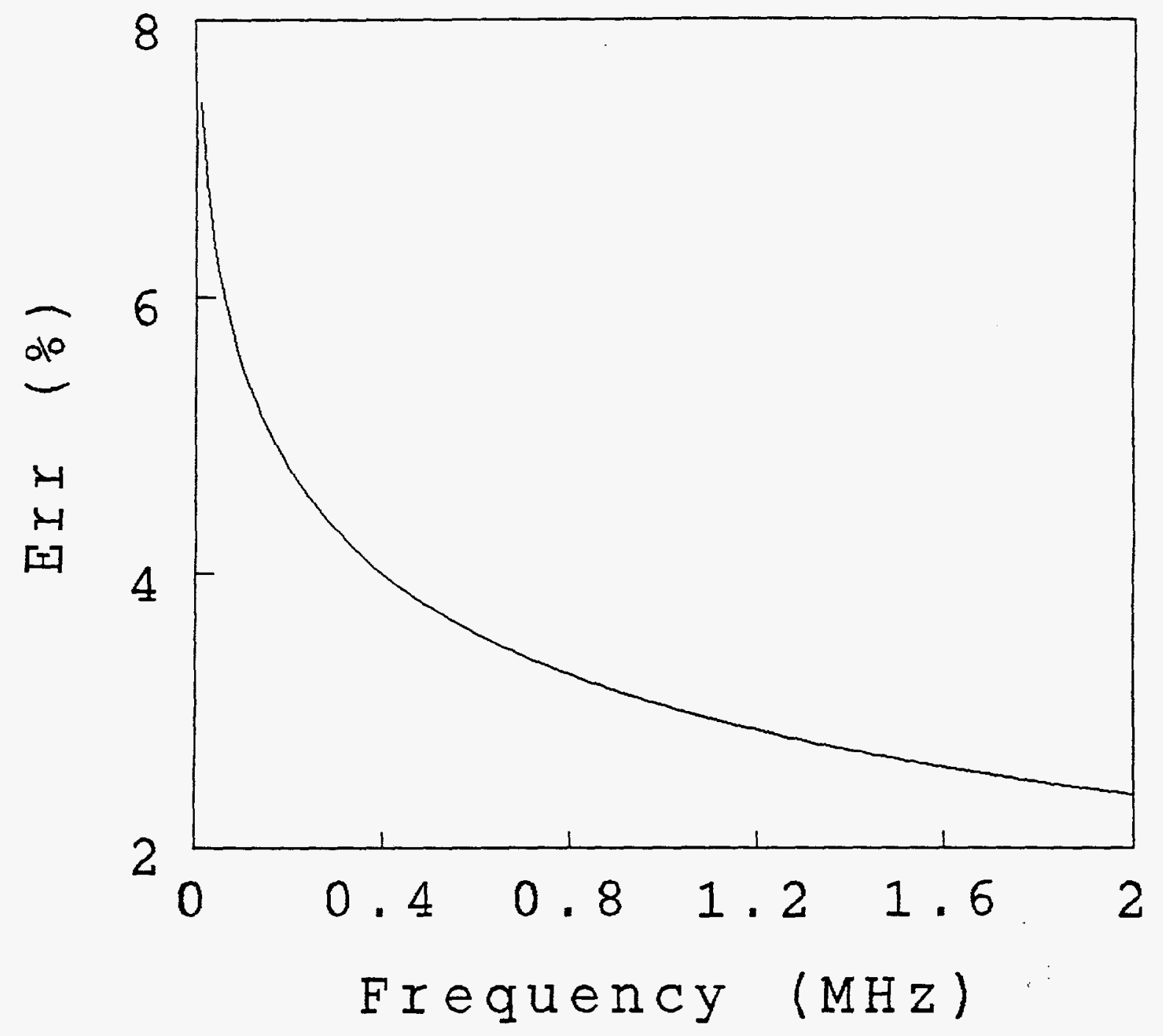

Fig. 5.5 A plot of the percent error between the inductance calculated for a perfectly conducting ground and the inductance calculated for an imperfect ground of $\sigma=1.6 \times 10^{-2}$. 
Note that the impedance $z_{g}$ does not have a circumflex over it to signify that it is in the time domain. $z_{q}$ is thus simply the inverse Fourier Transform of $\hat{z_{g}}$.

The derivation of the second Telegrapher's Equation starts by combining the continuity equation

$$
\oint_{s} J \cdot d S=-\frac{\partial Q}{\partial t}
$$

and Gauss's Law

$$
\oint_{s} D \cdot d s=Q
$$

to obtain

$$
\oint_{S} J \cdot d S+\frac{\partial}{\partial t} \oint_{S} D \cdot d S=0
$$

Integrating Eq. (5.29) over a cylindrical surface of length $\Delta x$ just outside the overhead conductor, dividing by $\Delta x$, and taking the limit as $\Delta x$ approaches zero, we get

$$
\frac{\partial I(x)}{\partial x}+\frac{\partial Q(x)}{\partial t}=0
$$

As with Eq. (5.8) above, the next step is to relate the terms in Eq. $(5.30)$ to more convenient parameters. The relation between the charge and the scattered voltage can be approximated as a constant coefficient equal to the capacitance $C$ per unit length of the line calculated 
assuming a perfect ground (Sunde, 1968). Sunde's approximation can be justified for the frequencies and parameters of interest by the same approximation used in the case of the inductance and shown in Figure 5.3 . This is so since position dependence of the radial electric field from an overhead line of charge is the same as for the magnetic flux from a line of current, inverse with respect to the distance to the wire. Making this substitution, we obtain the second Telegrapher's equation as follows

$$
\frac{\partial I(x)}{\partial x}+c \frac{\partial V_{s}(x)}{\partial t}=0
$$

\subsection{Frequency-Independent Ground Resistance Approximation}

Frequency dependent losses cause pulses to distort as they propagate down transmission lines. The following factors affect the severity of the distortion:

(1) The electric length of the path of the pulse: the longer the line the more distortion as different frequencies get attenuated and delayed unevenly .

(2) The frequency spectrum of the propagating pulse.

Using a constant, real value for the impedance of the ground, Rubinstein et al. (1989) obtained remarkably good results in analyzing voltages induced by distant lightning on the same test line used in the experiment described. The value of the constant resistance was arrived at by evaluating the frequency dependent impedance given by vance (1978) for the natural resonant frequency of the line, about $330 \mathrm{kHz}$, and then adjusting it by a trial and error method to match the data, a change of about 308 from the value at $330 \mathrm{kHz}$. We contend here that the success of the method utilized by Rubinstein et al. (1989) was only coincidental and that the resonance frequency of the line should not influence the frequency at which the constant equivalent resistance is estimated. In 
order to prove our point, let us conduct a thought experiment: think of two similar lines open-circuited at both ends, of lengths $450 \mathrm{~m}$ and 900 $\mathrm{m}$, respectively. Let us now think of a narrow pulse leaving one end of the shorter line towards the other end. Simultaneously, an identical pulse is launched from one end of the longer line. After $6 \mu$ sec each pulse has travelled a distance of $1800 \mathrm{~m}$ and they are both at the end from which they originated when the experiment began. Since both pulses have travelled the same total distance in the same time along physically similar lines of different lengths, it would be groundless to assume that the pulses will exhibit differences. One is led to conclude that the length of the line (and therefore its resonance frequency) does not play a role in the way the pulses decay due to the ground conductivity. Further, it has been pointed out by vance (private communication) that the approximate equation for the impedance per unit length used by Rubinstein et al. (1989) to calculate the constant resistance is a factor of two smaller than the one given in Vance (1987). This error, which originated as a typographical error in vance (1978) and which has been corrected in the 1987 edition, caused Rubinstein et al (1989), Master and Uman (1984), and Rachidi et al. (1990) to under-estimate the value of the resistance per unit length by a factor of two with respect to Vance's expression, and by a factor of four with respect to the new definition of surface impedance derived in the previous section. Rachidi et al. (1990) present theoretically calculated voltages using (a) a constant resistance obtained by trial and error, and (b) Vance's frequency domain impedance applied in the time domain using the convolution theorem. The authors note that the difference in the waveshapes and amplitudes obtained using these two approaches is negligible. 


\subsection{Exact Approach for Including the Finite Conductivity of the Ground}

In this section a method that permits the inclusion of the frequency dependent ground surface impedance in a time domain coupling code is presented. The development parallels that in Rachidi et al. (1990).

The objective is to incorporate into the time domain coupling code Eq. (5.19) which relates, in the frequency domain, the horizontal electric field at ground level to the current in the overhead wire. Eq. (5.19) is rewritten here for convenience

$$
\hat{E_{x}}(y=0, z=0) \approx \frac{(1+j)}{\pi h \sigma \delta} \hat{I}
$$

The product of two frequency domain functions can be expressed in the time domain in terms of a convolution integral as follows

$$
\hat{F}(w) \hat{G}(w) \Leftrightarrow \int_{-\infty}^{\tau} f(\tau) g(t-\tau) d \tau
$$

where $\hat{F}$ and $\hat{G}$ are the Fourier transforms of $f$ and $g$, respectively, and where the symbol $\Leftrightarrow$ has been used to signify that the quantity on each side is equivalent to the one on the other side if transformed to the other side's domain. We now define $\hat{G}(w)$ and $\hat{F}(w)$ as

$$
\hat{G}(w)=\frac{(1+j)}{\pi h \sigma \delta j w}
$$

and

$$
\hat{F}(w)=j w I(w)
$$

The inverse Fourier transform of Eq. (5.34) is given by

$$
g(t)=\frac{1}{\pi h} \frac{\left(\mu_{0}\right)^{1 / 2}}{\sigma \pi t)^{1 / 2}}
$$


while that of Eq. (5.35) is given by

$$
f(t)=\frac{\partial i(t, z)}{\partial t}
$$

Substituting Eqs. (5.36) and (5.37) into Eq. (5.33) we get

$$
\hat{F}(w) \hat{G}(w) \Rightarrow \int_{-\infty}^{t} \frac{\left(\mu_{0}\right)^{1 / 2}}{\pi h(\sigma \pi(t-\tau))^{1 / 2}} \frac{\partial i(\tau, z)}{\partial \tau} d \tau
$$

The singularity introduced by the $t-\tau$ term in the denominator of the integrand in Eq. (5.38) can be treated by writing the integral in two parts as follows

$$
\begin{gathered}
\hat{F}(w) \hat{G}(w) \Rightarrow \int_{-\infty}^{t-\Delta t} \frac{\left(\mu_{0}\right)^{1 / 2}}{\pi h(\sigma \pi(t-\tau))^{1 / 2}} \frac{\partial i(\tau, z)}{\partial \tau} d \tau \\
+\int_{\tau-\Delta \tau}^{t} \frac{\left(\mu_{0}\right)^{1 / 2}}{\pi h(\sigma \pi(t-\tau))^{1 / 2}} \frac{\partial i(\tau, z)}{\partial \tau} d \tau
\end{gathered}
$$

The second integral in Eq. (5.39) can be readily evaluated if the current derivative is assumed to vary slowly in the interval $\Delta t$ so that this derivative can be brought out of the integral sign. After doing this, Eq. (5.39) becomes

$$
\begin{aligned}
\hat{F}(w) \hat{G}(w) & \Leftrightarrow \int_{-\infty}^{t-\Delta t} \frac{\left(\mu_{0}\right)^{1 / 2}}{\pi h(\sigma \pi(t-\tau))^{y / 2}} \frac{\partial i(\tau, z)}{\partial \tau} d \tau \\
& +\frac{2\left(\mu_{0} \partial t\right)^{1 / 2}}{\pi h(\sigma \pi)^{1 / 2}} \frac{\partial i(t, z)}{\partial t}
\end{aligned}
$$


This approach has been implemented into a time domain computer code by Rachidi el al. (1990). They reported no significant effect on the calculated voltages when comparing this approach to the constant Iesistance approach used in Rubinstein et al. (1989) as discussed in the previous section. 


\section{CHAPTER VI}

\section{FIELD CALCULATIONS FOR THE COUPIING OF VERY CIOSE FIELDS TO OVERHEAD POWER LINES}

\subsection{The Vertical Field Over an Imperfectly Conducting Ground}

The electromagnetic fields from a vertical current source above a perfectly conducting ground plane can be obtained by adding the effects of the source and of the ground plane, the latter being expressible in terms of an image located symmetrically underneath the plane (Stratton, 1941). When the ground plane possesses a finite conductivity, its effect is no longer that of a "perfect" specular image. Nonetheless, the method of images can be used as a good approximation to find some components of the electromagnetic fields if the ground is a reasonable conductor, as discussed below. For frequencies lower than $1 \mathrm{MHz}$, with a ground conductivity of $1.6 \times 1^{-2}$ mho (the ground conductivity at the experimental site) and assuming a relative permittivity of 5 , the ground is a relatively good conductor $(0 / 2 \pi f e \geq 5.8)$. We argue that the vertical electric field and the horizontal magnetic field should be relatively insensitive to small perturbations in the image due to a finite but relatively high conductivity. This argument is suggested by the fact that, in the calculation of these components of the electromagnetic field, the effects of the source and its image interact constructively. In the case of the horizontal component of the electric field, however, a small change in the fields produced by the image can produce large changes in the total field since the fields from the source and from the image interact destructively, as will be further discussed in the next section.

The exact solution of the problem demands the use of the so called "Sommerfeld approach" (1909). Sommerfeld (1909) attacked the problem of 
an infinitesimal vertical current element above the ground plane. He utilized Hertz potentials to find possible solutions within each semispace and applied boundary conditions at the interface. The simplicity of his development may seem obscured by the mathematics involved in the manipulation of the inhomogeneous solution to the Hertz potential equation for the top semi-space, which he expressed as an integral in terms of Bessel functions of the first kind. Due to a sign error, the results of Sommerfeld (1909) are incorrect, a fact pointed out by Norton $(1935,1937)$. The correct results and a detailed derivation is found in many textbooks (i.e., stratton, 1941; Wait, 1985).

Although Sommerfeld's solution has been programmed into computer codes (e.g., NEC-2 developed at Lawrence Livermore National Laboratory), these programs are not directly useful for return stroke field calculations since they are written only for monochromatic sources. This requires that the effect of every frequency component of the current at every height be calculated. Then all of the outputs would have to be appropriately added together and transformed back to the time domain, after which the fields at only one of the many field points needed for the coupling calculations would have been determined. An approximate technique that allows us to calculate the needed fields by modifying the fields obtained with a perfectly-conducting ground model will be presented in section 6.2.2.

\subsection{The Horizontal Field Over an Imperfectly \\ conducting Ground}

\section{2 .1 Use of the Wavetilt for the Calculation of Distant Horizontal Electric Fields}

In 1984 at the NASA Kennedy Space Center, The University of Florida Iightning Research Group carried out the first measurements of the three perpendicular components of the electric field from distant lightning 
return strokes (Thomson et al., 1988a,b). Further, the horizontal component of the electric field was calculated from the measured vertical field by means of the wavetilt formula using a time domain technique developed by Master and Uman (1984), as a test of the wavetilt approach. A comparison of the calculated and the measured horizontal field waveforms revealed very good agreement in both the waveshapes and the amplitudes of the fields.

The wavetilt formula is the ratio, in the frequency domain, of the horizontal to vertical electric field components of a plane wave for grazing incidence. A simple derivation for the case of a single layer semi-space follows.

Figure 6.1 shows the geometry of the problem. The total horizontal field at $z=0$ is the sum of the horizontal components of the incident and the reflected waves and is therefore expressible as

$$
E_{b}=E_{1} \cos \left(\alpha_{1}\right)[1-\Gamma]
$$

where $\Gamma$ is the reflection coefficient for parallel polarization, and $\alpha_{i}$ is the incident angle. The vertical field, on the other hand, is

$$
E_{v}=E_{f} \sin \left(\alpha_{1}\right)[1+\Gamma] \text {. }
$$

Dividing Eq. (6.1) by Eq. (6.2), we obtain

$$
W T=\cot \left(\alpha_{i}\right)[1-\Gamma] /[1+\Gamma]
$$

Eq. (6.3) can be written in terms of the electrical parameters of the media by using the expression for the Fresnel reflection coefficient

$$
\Gamma=\frac{z_{1} \cos \left(\alpha_{1}\right)-z_{2} \cos \left(\alpha_{t}\right)}{z_{1} \cos \left(\alpha_{1}\right)+z_{2} \cos \left(\alpha_{t}\right)}
$$

where $z_{n}$, the impedance of medium $n$, is $\left(\mu_{n} / \epsilon_{n}\right)^{1 / 2}$, and $\alpha_{t}$ is the transmission angle. After these substitutions, Eq. (6.3) becomes 


$$
W T=\frac{\cos \left(\alpha_{t}\right)}{\sin \left(\alpha_{1}\right)\left(\epsilon_{x}+\sigma / j \omega \varepsilon_{0}\right)^{1 / 2}}
$$

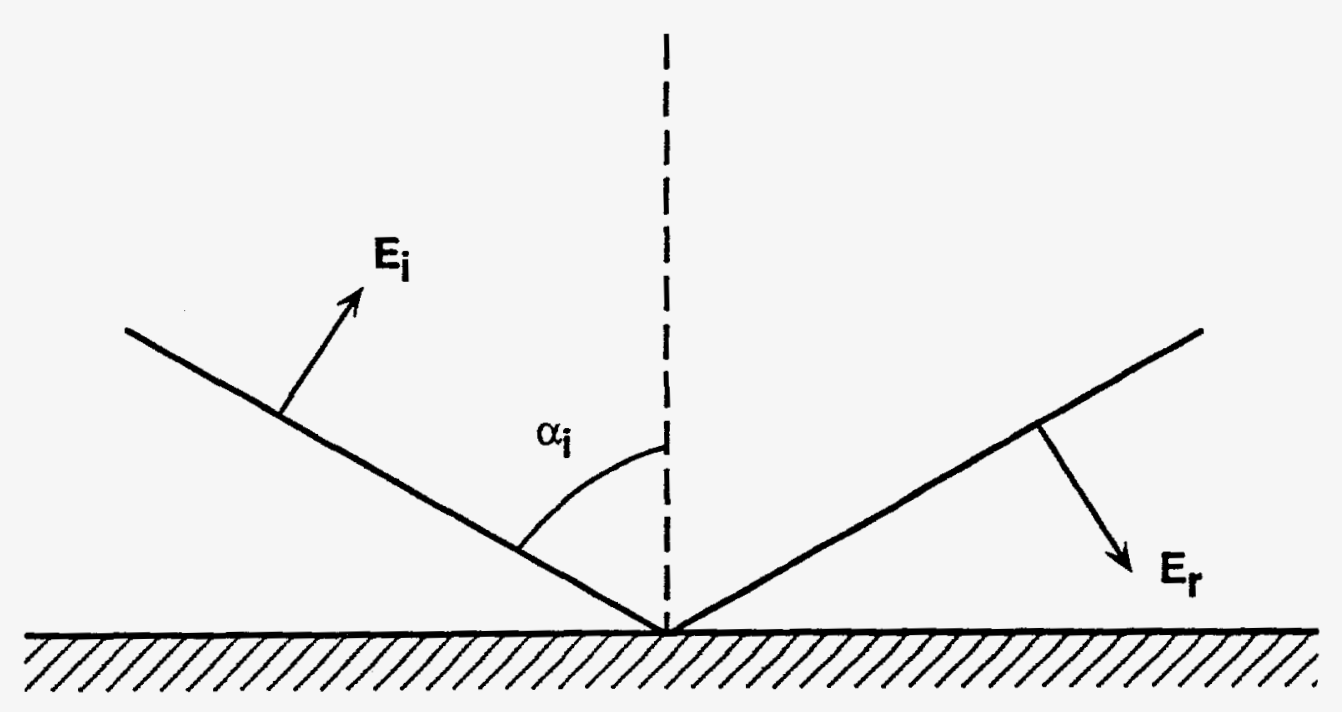

Fig. 6.1 Definition of the geometry used in the derivation of the wavetilt formula. 
Now, for the case of a plane wave going from air to either a good conductor $\left(\sigma / j \omega \epsilon_{2} \gg 1\right)$ or to a conductor with a relative permittivity that is much larger than $1, \cos \left(\alpha_{t}\right)$ can be proven to be approximately 1 regardless of the incident angle $\alpha_{i}$, by applying Snell's Law, $\left(\epsilon_{0}\right)^{1 / 2} \sin \left(\alpha_{i}\right)$ $=\left(\hat{\varepsilon}_{2}\right)^{1 / 2} \sin \left(\alpha_{t}\right)$, where, neglecting non-ohmic losses, $\hat{\varepsilon}_{2}=\varepsilon_{2}+\sigma / j \omega$ is the complex permittivity of the conducting medium. We first solve for $\sin \left(\alpha_{t}\right)$

$$
\sin \left(\alpha_{t}\right)=\sin \left(\alpha_{1}\right)\left(\epsilon_{0} / \hat{\epsilon}_{2}\right)^{1 / 2}
$$

The cosine of $\alpha_{t}$ is therefore

$$
\cos \left(\alpha_{t}\right)=\left(1-\sin ^{2}\left(\alpha_{1}\right)\left(\epsilon_{0} / \hat{\epsilon}_{2}\right)\right)^{1 / 2}
$$

Now, recalling that $\hat{\varepsilon}_{2}=\varepsilon_{2}+\sigma / j \omega$, we can rewrite Eq. (6.6) as follows

$$
\cos \left(\alpha_{t}\right)=\left(1-\sin ^{2}\left(\alpha_{i}\right) \frac{\epsilon_{0}}{\left(\varepsilon_{2}+\sigma / j \omega\right)}\right)^{1 / 2}
$$

which, in turn, can be rewritten as

$$
\cos \left(\alpha_{t}\right)=\left(\frac{1+\sigma / j \omega \varepsilon_{2}-\sin ^{2}\left(\alpha_{1}\right) \varepsilon_{d} / \varepsilon_{2}}{\left(1+\sigma / j \omega \varepsilon_{2}\right)}\right)^{1 / 2}
$$

Finally, since $\left.\left(1-\sin ^{2}\left(\alpha_{i}\right) \varepsilon_{0}\right) / \epsilon_{2}\right)<1$, and since for a good conductor $\sigma / j \omega \varepsilon_{2} \gg 1$, we conclude that $\cos \left(\alpha_{t}\right) \sim 1$. We can readily arrive at the same conclusion if the relative permittivity $\epsilon_{2} / \epsilon_{0} \gg 1$. Further, for grazing incidence, $\alpha_{i}=90^{\circ}$. Making use of these approximations, we obtain the following expression for the wavetilt formula

$$
W T=\frac{1}{\left(\epsilon_{I}+\sigma / j \omega \epsilon_{0}\right)^{1 / 2}}
$$

where $\epsilon_{\mathrm{r}}=\epsilon_{2} / \epsilon_{0}$

The technique of Master and Uman (1984) enables us to apply Eq. (6.10) to time domain vertical electric field waveforms in order to obtain the horizontal field. In this technique, the "Laplace" transform 
is applied to a piecewise linear approximation of the vertical electric field obtained by expressing the waveform as a sum of appropriately delayed ramps. The result is multiplied by the wavetilt formula, and the time domain horizontal field is obtained as a sum of time delayed responses by taking the inverse Laplace transform of this product. The advantage of expressing the time domain vertical electric field as a sum of ramps is threefold: (I) the "Laplace transform"-multiplication"inverse Laplace transform" operation described above yields a closed analytical expression for each ramp, (2) the output is not affected by whether or not the input waveform has decayed to zero within the time window considered, as in the case of frequency domain techniques, and (3) it is easily implemented for computer calculations. This technique is described in detail in Master (1982), Master and Uman (1984), and Rubinstein (1986).

\subsubsection{The Calculation of Close Horizontal Electric Fields}

In this section, we develop a technique for calculating the horizontal electric field close to a lightning needed for the coupling calculations. Our goal is to find the horizontal electric field at a height $h$ (the height of the overhead wire) from the return stroke current. We will only deal with one elemental segment of the lightning channel since superposition allows that the results be applicable to the complete channel. The pertinent geometry is shown in Figure 6.2. The first step is to integrate the Ampere-Maxwell equation given below

$$
\oint H \cdot d 1=\iint J \cdot d S+\frac{\partial}{\partial t} \iint D \cdot d S
$$

around an infinitely long rectangle of differential width rd $\phi$ extending from $z=0$ (ground level) to $z=-\infty$, as shown in Figure 6.2. Using the facts that (1) the vertical component of the magnetic field is identically 


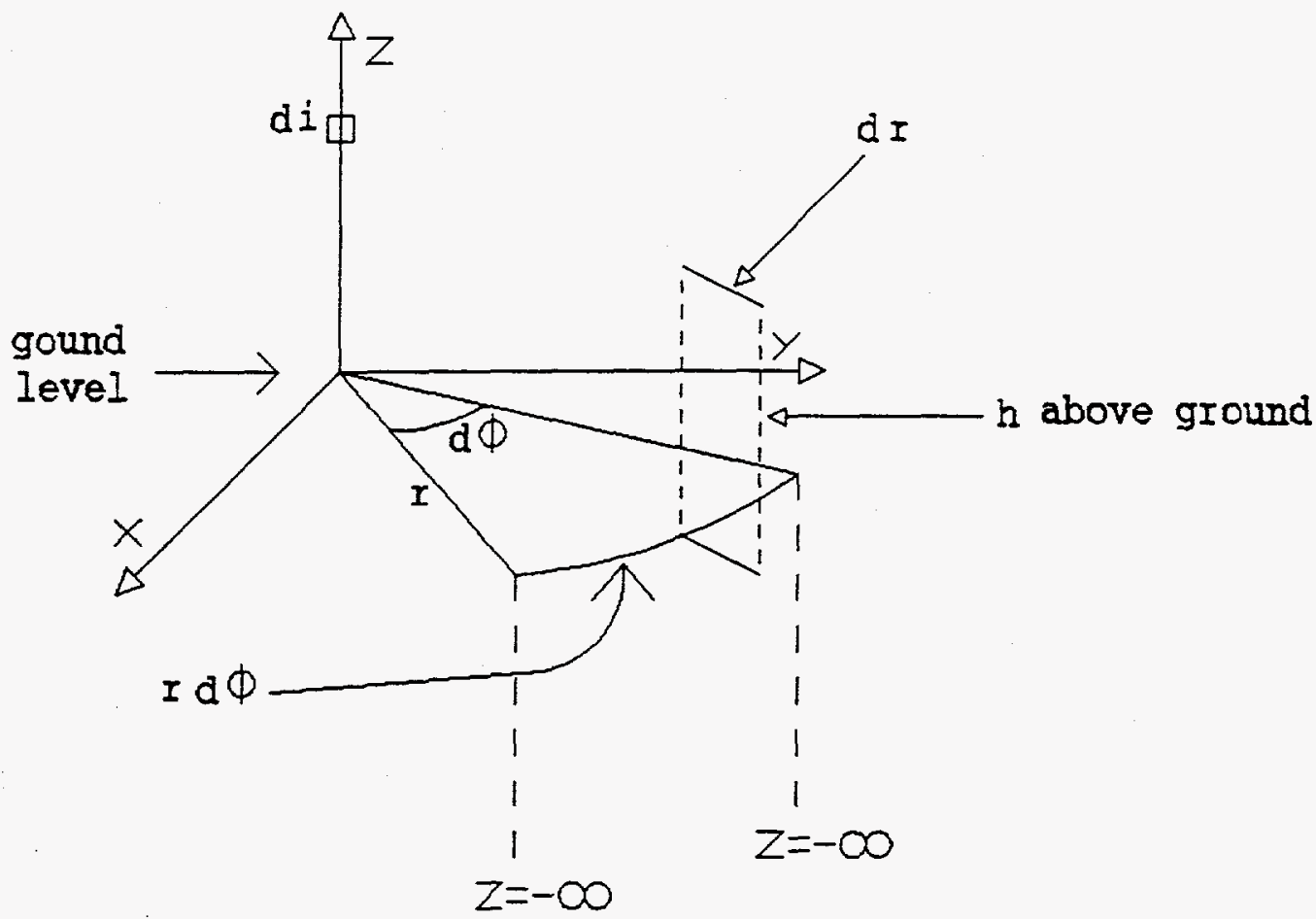

Fig. 6.2 Definition of the geometry used in the calculation of the horizontal electric field over an imperfectly conducting ground. 
equal to zero for a vertical dipole over an imperfect ground (Sommerfeld, 1909), and (2) all the field components are zero at $z=-\infty, E q \cdot(6.11$ ) can be written as

$$
I d \phi H_{\phi}(z=0, I)=-d I
$$

where $\mathrm{dI}$ is the conduction current flowing through the area of the rectangle of integration in the positive $r$ direction, and where we have assumed $\sigma>>\omega e$ and have thereby neglected the displacement current $\partial D / \partial t$. This assumption is valid for frequencies below $2 \mathrm{MHz}$ and for the electrical parameters of the ground found at the site of the experiment, $\sigma=1.6 \times 10^{-2} \mathrm{mho} / \mathrm{m}$ and $\epsilon_{x}$ of the order of 10 for wet earth (zenneck, 1915). Weyl (1919) expressed the results of sommerfeld (1909) for the fields from an elemental dipole over an imperfect ground as a bundle of plane waves reflected and refracted by the surface of the ground with real and complex angles of incidence. With the knowledge that plane waves refracted at the air-earth interface have a functional dependence with depth given (at least for real incident angles) by

$$
E_{I}(z)=E_{Y}(z=0) e^{(j+1) z / 8}
$$

where $\delta$, the skin depth factor, is given by $1 /(\pi \mu \sigma f)^{1 / 2}$, we can find the current on the right hand side of Eq. (6.12) by multiplying Eq. (6.13) above by the conductivity $\sigma$ and the differential length rd $\phi$ in order to obtain the linear current density, and then integrating it from $z=-\infty$ to $z=0$. The result of this integration is

$$
d I=\sigma E_{I}(z=0, I) \frac{\delta}{(1+j)} r d \phi
$$

Substituting Eq. (6.14) into Eq. (6.12) and solving for the electric field, we obtain

$$
E_{z}(z=0, r)=-H_{\phi}(z=0, r) \frac{(1+j)}{\sigma \delta}
$$


which can be rearranged as an expression of the so-called surface impedance, $E_{x}(z=0) / H_{\phi}(z=0)=-(1+j) / 8 \sigma$. Assuming once again that the horizontal magnetic field for an imperfectly conducting ground can be approximated as that obtained in the infinite conductivity case, we can rewrite Eq. (6.15)

$$
E_{x}(z=0, I)=-H_{\phi p}(z=0, I) \frac{(1+j)}{\sigma \delta}
$$

where the subindex $p$ has been introduced to indicate the fields obtained assuming a perfect ground.

In order to find the horizontal electric field at the height of the line we integrate Faraday's equation

$$
\oint E \cdot d l=-\frac{\partial}{\partial t} \iint B \cdot d S
$$

around a rectangle of width dr extending up from $z=0$ to $z=h$ (see Figure $6.2)$. The result is shown in Eq. (6.18) below

$$
\begin{aligned}
& E_{r}(z=h, r) d r-E_{Y}(z=0, r) d r+\int_{0}^{h} E_{z}(z, r) d z \\
& -\int_{0}^{h} E_{z}(z, r+\delta r) d z=-d r \frac{\partial}{\partial t} \int_{0}^{h} B_{\phi}(z, r) d z
\end{aligned}
$$

Now, integrating Faraday's equation around the same rectangular path for the case of a perfectly conducting ground we obtain 


$$
\begin{gathered}
E_{x p}(z=h, I) d I+\int_{0}^{h} E_{z p}(z, I) d z \\
-\int_{0}^{h} E_{z p}(z, I+\delta r) d z=-d r \frac{\partial}{\partial t} \int_{0}^{h} B_{\phi p}(z, I) d z
\end{gathered}
$$

where, once again, the subindex $p$ has been added to signify the fields for the case of a perfectly conducting ground and where the horizontal electric field at the ground has been set to zero.

In Eq. (6.19), we again approximate the vertical electric field $E_{2 p}(z, r)$, and the horizontal magnetic field $B_{\phi p}(z, r)$ for the case of a perfectly conducting earth as the fields for the case of an imperfect ground $E_{z}(z, r)$ and $B_{\phi}(z, r)$, respectively. Making these substitutions into Eq. (6.19) and combining the resulting equation with Eq. (6.18), we obtain

$$
E_{Y}(z=h, I)=E_{Y}(z=0, I)+E_{r p}(z=h, I)
$$

Finally, substituting Eq. (6.16) into Eq. (6.20), we obtain the result sought

$$
E_{x}(z=h, r)=-H_{\phi p}(z=0, I) \frac{(I+j)}{\sigma \delta}+E_{x p}(z=h, r)
$$

The importance of this result is evident when we note that the horizontal electric field at line height can be obtained from the electric and magnetic fields of the perfectly-conducting ground model. For large values of $\mathrm{r}$, Eq. $(6.21)$ should produce an expression of the wavetilt formula, Eq. (6.10), given in section 6.2.1. This can be readily shown as follows: for large values of $\mathrm{r} / \mathrm{h}$, only the radiation components of the fields will be appreciable. $E_{x p}(z=h, r)$ on the right-hand side of 
Eq. 6.21) can be neglected since it decays more rapidly than $\mathrm{H}_{\phi \mathrm{p}}(z=0, r)$. Making this approximation and solving for the ratio of $E_{x p}(z=h, r)$ to $\mathrm{H}_{\phi P}(z=0, r)$ we obtain

$$
\frac{E_{Y}(z=h, I)}{H_{\phi D}(z=0, I)} \propto-\frac{(I+j)}{\sigma \delta}, \quad I / h>>1
$$

Where we see that, under these conditions, the ratio of $\mathrm{E}_{r}$ to $\mathrm{H}_{\phi p}$ becomes nearly independent of $h$. The radiation components of the vertical electric field and the horizontal magnetic field are related by the wave impedance $\mathrm{E}_{z}(z=0, r) / \mathrm{H}_{\phi \mathrm{p}}(z=0, r)=-\left(\mu_{0} / \epsilon_{0}\right)^{1 / 2}$. Substituting this relation into Eq. (6.22) in order to eliminate the magnetic field, and solving for the ratio of the horizontal to the vertical components of the electric field, we obtain

$$
\frac{E_{z}(z=h, r)}{E_{z p}(z=0, r)}=\left(\epsilon_{0} / \mu_{0}\right)^{1 / 2} \frac{(1+j)}{\sigma \delta}
$$

which can be rewritten as

$$
\frac{E_{x}(z=h, I)}{E_{z p}(z=0, I)}=\left(j \omega \epsilon_{0} / \sigma\right)^{1 / 2} \text {. }
$$

$\mathrm{Eq} \cdot(6.24)$ is seen to be equal to $\mathrm{Eq} \cdot(6.10)$ for the case where $\left(\sigma / \mathrm{j \omega \epsilon _{0 }}\right)^{1 / 2}$ $>\epsilon_{\mathrm{r}}$.

Figure 6.3 shows examples of horizontal electric field calculated $20 \mathrm{~m}$ and $450 \mathrm{~m}$ away from the lightning channel using this technique and the Diendorfer-Uman lightning return stroke model (Diendorfer and Uman, 1990). The parameters chosen were: the front time constants of the core and corona currents at ground, $0.07 \mu \mathrm{sec}$ and $2.0 \mu \mathrm{sec}$, respectively, (2) the decay time constants of the core and corona currents at ground, 2.2 $\mu$ sec and $20.0 \mu \mathrm{sec}$, respectively, (3) the amplitude of the core and corona currents at ground, $5.25 \mathrm{kA}$ and $3.75 \mathrm{kA}$, respectively, 
(a)
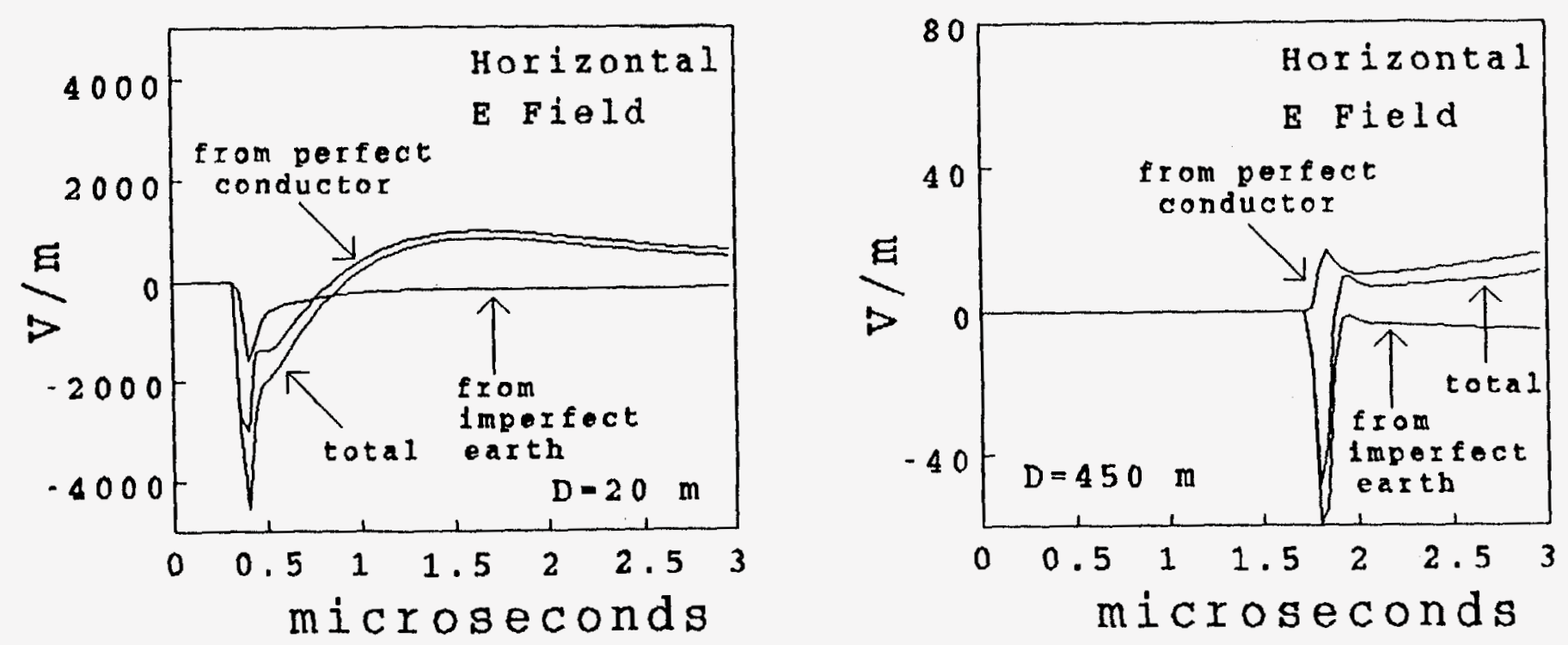

Fig. 6.3 Horizontal field calculated over an imperfectly conducting ground for distances of $20 \mathrm{~m}$ and $450 \mathrm{~m}$. In (a) the field is plotted in a $3 \mu \mathrm{sec}$ scale and in (b) in a $25 \mu \mathrm{sec}$ scale. The field has been separated into two components in accordance with Eq. (3.77). The front time constants of the core and corona currents at ground are $0.07 \mu \mathrm{sec}$ and $2.0 \mu \mathrm{sec}$, respectively. The decay time constants of the core and corona currents at ground are $2.2 \mu \mathrm{sec}$ and $20.0 \mu \mathrm{sec}$, respectively. The amplitudes of the core and corona currents at ground are 5.25 $\mathrm{kA}$ and $3.75 \mathrm{kA}$, respectively. The core and corona discharge time constants, 0.1 usec and $6.0 \mu \mathrm{sec}$, respectively. The striking height, $20 \mathrm{~m}$. 
(b)
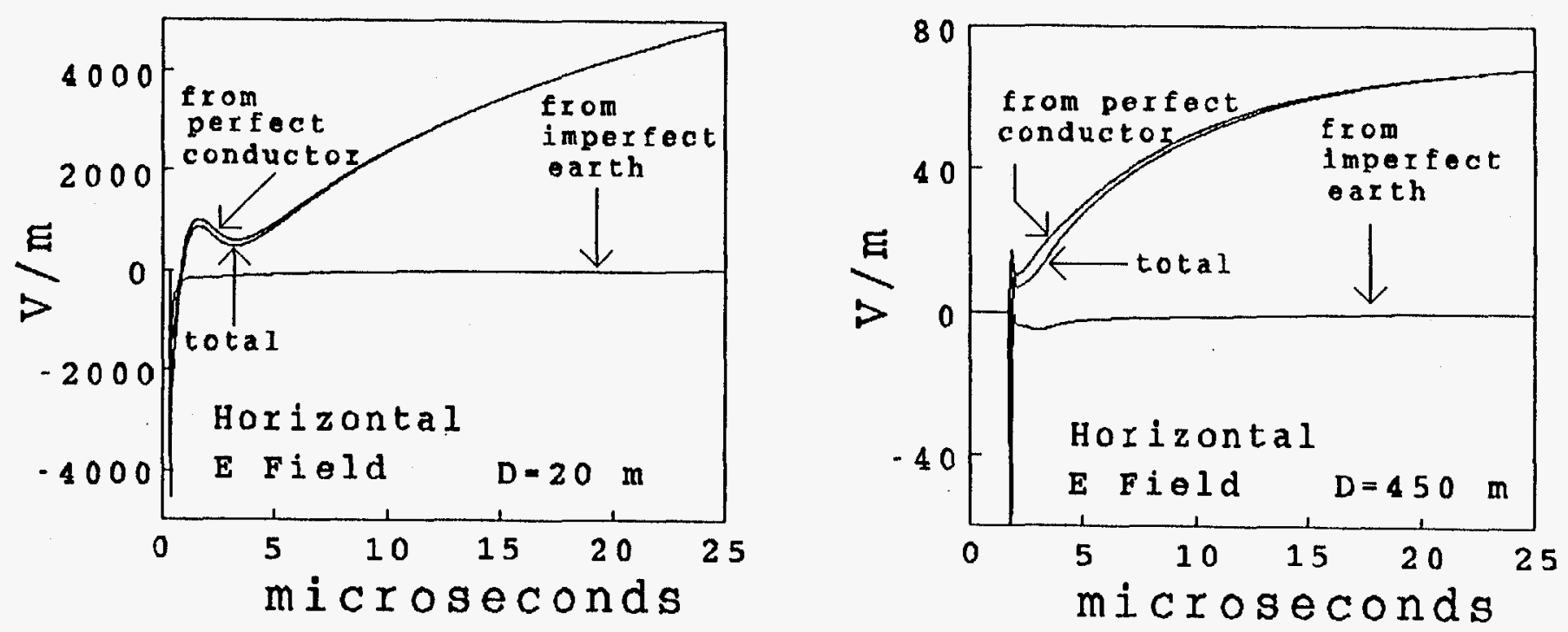

Figure 6.3 continued 
(4) the core and corona discharge time constants, $0.1 \mu \mathrm{sec}$ and $6.0 \mu \mathrm{sec}$, respectively, and (5) the striking height, $20 \mathrm{~m}$. For both distances, a sharp negative peak is seen to precede a field change that appears similar to the vertical electric component of the return stroke electric field. This fast change is due, in part, to the finite conductivity of the ground. 
CHAPTER VII

COMPARISON OF THEORY AND EXPERIMENTAL RESULTS
FOR VERY CLOSE IIGHTNING

\subsection{Introduction}

In this chapter we apply the time domain coupling theory presented in Chapter $\mathrm{V}$ to the close lightning data characterized in Chapter III. To this end, we first compute the return stroke electric and magnetic fields from the artificially initiated lightning discharges by means of the Diendorfer-Uman lightning return stroke model (Diendorfer and Uman, 1990 ) and the field calculation techniques described in Chapter VI. We then use these fields as inputs to a computer code that calculates the desired voltages by way of the time domain coupling approach of Agrawal et al. (1980). Examination of waveforms calculated using the procedure described above shows that, by choosing the appropriate channel-base current (the actual currents measured in 1986 exhibited characteristics, such as bipolarity, that have not been observed in any other triggered lightning current data base and hence we believe them to be erroneous), it is possible to obtain fair agreement in wave shape between the numerically calculated voltages and the measured oscillatory voltages. Some improvement in the wave shape agreement is obtained by modeling corona effects. Agreement with the measured vertical electric field intensities can also be obtained, although a given channel-base current does not necessarily yield the best wave shape agreement for the electric field intensity and for the oscillatory voltages simultaneously. The rise time and decay time of the channel-base currents used to obtain the best matches were found to be consistent with the data base available in the 
literature. Impulsive voltages, whose peak amplitudes are five to ten times larger than oscillatory voltage peak amplitudes, probably caused flashovers to occur at the line terminations. The most likely flashover patterns between the voltage dividers and the resistive terminations are discussed in Section 7.3 where we model the impulsive voltages by simulating these non-linear effects in our time domain computer program. The level of wave shape agreement obtained supports the flashover hypothesis. To obtain amplitude agreement at both ends of the line, however, unreasonably large channel-base currents with peak amplitudes in excess of $200 \mathrm{kA}$ (higher than the $60 \mathrm{kA}$ maximum reported in the literature for triggered lightning) need to be used.

\subsection{Method of Analysis}

To find the electric and magnetic fields needed for the coupling calculations, we have used the Diendorfer-Uman return stroke model (Diendorfer and Uman, 1990). This model predicts with good accuracy all the salient features of electric and magnetic field measurements from natural as well as artificially initiated lightning for lightning beyond about $1 \mathrm{~km}$, the range for which these measurements exist. In addition, it is a physically reasonable and analytically simple model, and it is the only available model that can explain the differences obtained between optically measured return stroke speeds, theoretically calculated transmission line speeds from field and current measurements, and transmission line speeds from field and current derivatives.

In order to calculate the appropriate fields, knowledge of the actual channel-base return stroke current is desirable. However, as mentioned in Section 7.1, the measured channel-base currents for the 1986 experiment display an atypical bipolar shape not seen in any of the channel-base currents measured up to date, which leads us to believe that 
the current measuring system malfunctioned. In the absence of the actual currents, we have used return stroke current waveforms with different values for various current parameters in an attempt to simultaneously obtain fields consistent with the vertical electric field measurements at $500 \mathrm{~m}$ and voltages consistent with the measured voltages at either end of the test line.

The current in the Diendorfer-Uman model is specified in terms of two currents components called the "core current" and the "corona current". We will refer to the first of these two currents as "current $A "$ and to the second as "current B". Current $A$ is "faster" than current $B$, and it determines the rise time characteristics of the current waveform; while current B is "slower", and it determines the decaying portion of the current waveform after the peak (see Figure 7.1). The total return stroke current is expressed as

$$
i(0, t)=I_{A} \frac{\left(\frac{t}{\tau_{1 A}}\right)^{2}}{\left(\frac{t}{\tau_{1 A}}\right)^{2}+1} e^{-\frac{t}{\tau_{2 A}}}+I_{B} \frac{\left(\frac{[t]}{\tau_{1 B}}\right)^{2}}{\left(\frac{[t]}{\tau_{1 B}}\right)^{2}+1} e^{-\frac{[t]}{\tau_{2 B}}}
$$

where $i(0, t)$ is the return stroke current at the base of the channel, $I_{\mathrm{A}}$ and $I_{B}$ are the amplitude factors of the core and corona currents, respectively, $\tau_{1 \mathrm{~A}}$ and $\tau_{18}$ are the front time constants of the core and corona currents, respectively, $\tau_{2 \mathrm{~A}}$ and $\tau_{2 B}$ are the decay time constants of the core and corona currents, respectively, and the brackets around the time $t$ in the corona current term indicate that the corona current starts at a delayed time $t_{\text {delay }}$ and not at $t=0$.

In what follows, we present an algorithm with which, given five current parameters: (1) the 10-908 rise time $t_{\text {risef }}$ of the faster part of 


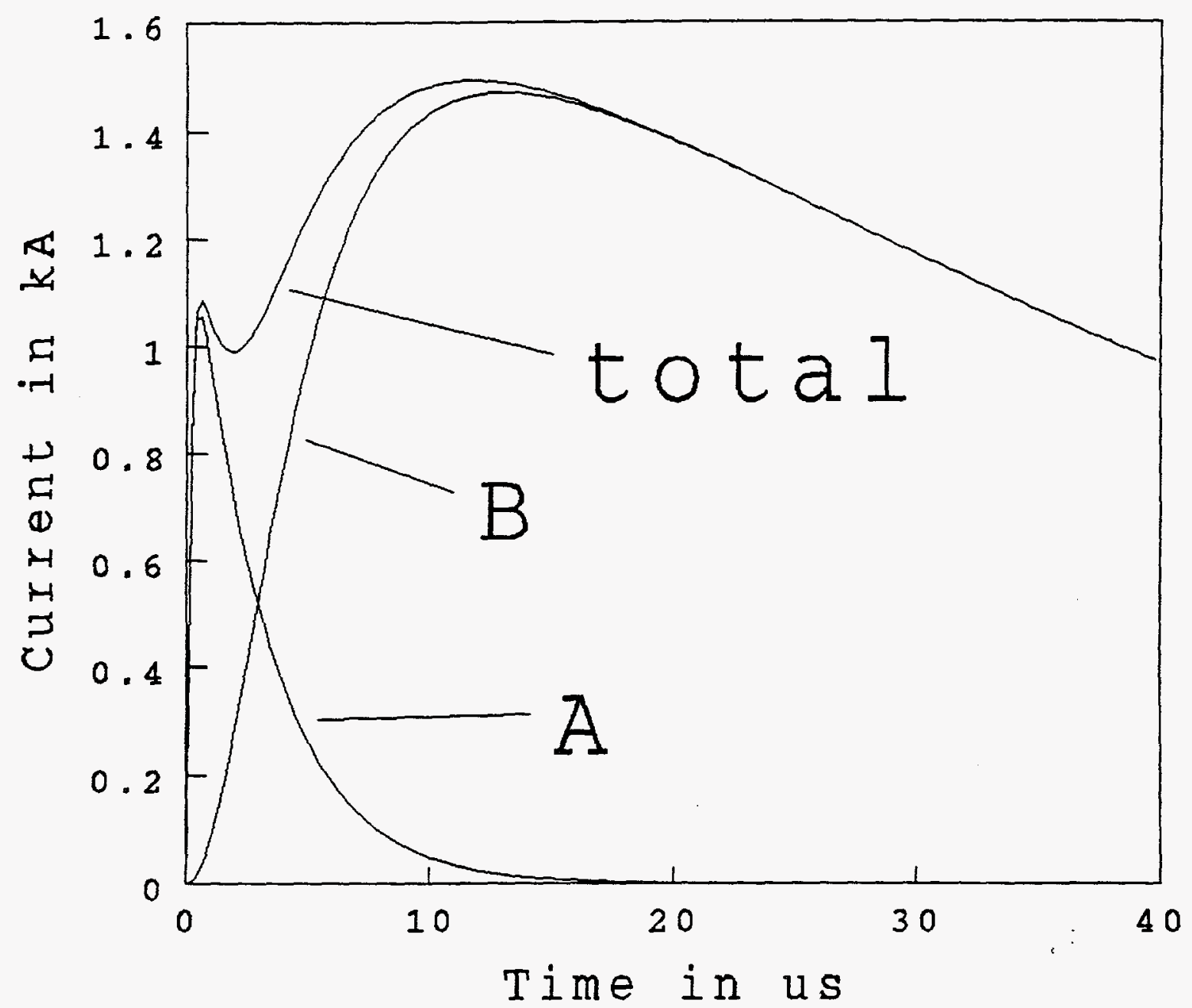

Fig. 7.1 A channel-base current used in the analysis showing the separation into currents $A$ (core current) and current $B$ (corona current). 
the rise to peak (hereafter called the transition) in the channel-base current, (2) the decay time constant $\tau_{d}$ of the channel-base current, (3) the ratio of the peak corona current to the peak core current $i_{\max } / i_{\max }$ (different from the ratio of $I_{B}$ to $I_{A}$ ), (4) the 10-908 rise time $t_{\text {riec }}$ of the corona current, and (5) the peak amplitude of the channel-base current, one can calculate the coefficients $\left(I_{A}\right.$ and $I_{B}$ ) and the time constants $\left(\tau_{1 A}, \tau_{2 A}, \tau_{1 B}\right.$, and $\left.\tau_{2 B}\right)$ which determine the current:

1. The decay parameter $\tau_{2 B}$ can be readily determined by noting that, for large times, the total current is dominated by the corona current $B$ and by noting that current $B$ reduces to a simple exponential as $\left(t / \tau_{1 B}\right)^{2}>1$ (see Eq. (7.1)). With these approximations, we can write $\tau_{2 B} \sim \tau_{d}$. 2. The amplitude parameter $I_{B}$ of the corona current is initially set to 1. This parameter will be scaled later to obtain the desired current peak amplitude.

3. Once $\tau_{2 B}$ and $I_{B}$ are set, the rise time parameter $\tau_{1 B}$ of the corona current is found by way of an iterative process as follows:

(a) Set the rise time parameter $\tau_{1 B}$ equal to the corona rise time $t_{\text {risec }}$.

(b) Compute the 10-908 rise time of the corona current numerically.

(c) Compute the percent error between the numerically calculated rise time and the desired corona rise time $t_{\text {rieoc }}$. If this error is less than 58 in absolute value, end the iterative process and continue to point 4. , below. If the error is greater than 58 , adjust $\tau_{18}$ by an amount equal to the percent error encountered and repeat (b) and $(c)$.

4. Once $\tau_{18}$ has been determined, the peak amplitude $i_{B \max }$ of current $B$ (which is different from the amplitude coefficient $I_{B}$ ) is calculated numerically.

5. With the knowledge of the peak amplitude of the corona current $i_{\text {Bnax }}$ and of the $i_{\text {max }} / i_{\text {max }}$ ratio (one of the five known parameters), the peak amplitude of the core current, $i_{\text {naxax }}$ is calculated. 
6. The decay time constant of the core current, $\tau_{2 \mathrm{~A}}$, is set to $2 \mu \mathrm{sec}$ and the delay time of the corona current is set to $1 \mu \mathrm{sec}$ (these values are consistent with the values used by Diendorfer and Uman (1990) to obtain the best match with experimental electric fields from triggered lightning).

7. $I_{\mathbf{A}}$ and $\tau_{1 \lambda}$, the only two parameters that remain to be found are calculated by means of the following iterative procedure similar to the one used to find $\tau_{1 \mathrm{~B}}$ in step 3 , above:

(a) Set the rise time parameter $\tau_{1 \mathrm{n}}$ equal to the transition 10-908 rise time $t_{r i e f}$ and set the amplitude coefficient $I_{n}$ equal to the value of $i_{\text {max }}$ calculated in step 5. , above.

(b) Compute both the 10-908 rise time of the transition of the channel base current and the amplitude of the core current numerically.

(c) Compute the percent errors between the numerically calculated rise time and the desired rise time $t_{r i n e f}$ and between the numerically calculated peak amplitude of the core current and the desired peak amplitude $i_{\text {anax }}$. If each of these errors is less than 58 in absolute value, stop the iterative process and proceed to 8 ., below. If either or both of the errors are greater than 58 , adjust $\tau_{11}$ and $I_{\lambda}$ by amounts equal to the percent errors encountered for the rise time $t_{\text {rieef }}$ and the current amplitude $i_{\max ,}$ respectively, and repeat (b) and $(c)$.

8. Find the peak amplitude of the channel base current numerically and adjust it to the desired amplitude by multiplying both amplitude factors $I_{A}$ and $I_{B}$ by the appropriate factor.

Currents with different rise times, decay times, corona current rise times, and $i_{\max } / i_{\max }$ ratios were obtained by this technique and were used to calculate waveforms of the vertical electric field intensity at the ground, the horizontal electric field intensity at line height, and the horizontal magnetic flux intensity at the ground using a perfectly conducting ground model. The Diendorfer-Uman model was applied for these calculations with different values for the return stroke speed, the corona discharge time constant, and the core discharge time constant. The 
horizontal component of the electric field intensity and the horizontal magnetic flux density were combined using the technique developed in Chapter IV in order to obtain the horizontal field at line height with a finite earth conductivity of $1.6 \times 10^{-2}$, the measured effective value at the experiment site (Rubinstein et al., 1989). The vertical electric field intensity was also calculated at $500 \mathrm{~m}$ for the purpose of comparing it to the measured fields. The fields thus calculated were used as inputs to a time domain computer code based on the approach of Agrawal et al. (1980) to calculate the voltages at both ends of an overhead line with the same geometry as that of the actual line used in the experiment.

\section{$\underline{7.3 \quad \text { Discussion }}$}

We have found a set of currents that yields fair agreement between the measured and the calculated voltages at either end of the test line for the case of the oscillatory voltages. These currents together with the vertical component of the electric field intensity calculated at 500 $\mathrm{m}$ and the resulting voltage simulations at the east and west ends of the line are shown in Figures 7.2 through 7.7. For all of the oscillatory voltages, agreement was obtained by using the following values for the Diendorfer-Uman return stroke parameters: return stroke speed $=150$ $\mathrm{m} / \mu \mathrm{sec}$, core current discharge constant $=0.1 \mathrm{\mu sec}$, corona current discharge constant $=6.0 \mathrm{\mu sec}$. The value of the return stroke speed is consistent with optical measurements reported by Idone et al. (1984) and the values of the discharge time constants are the ones used by Diendorfer and Uman (1990). The agreement between measured and calculated voltages is better for those events that occurred on day 240 than for those on day 232: In the oscillatory voltages of day 232, the first negative pulse measured at the east end of the line is always smaller than the second negative pulse (see Figures $3.5 \mathrm{~b}$ and $3.5 \mathrm{f}$ ). 


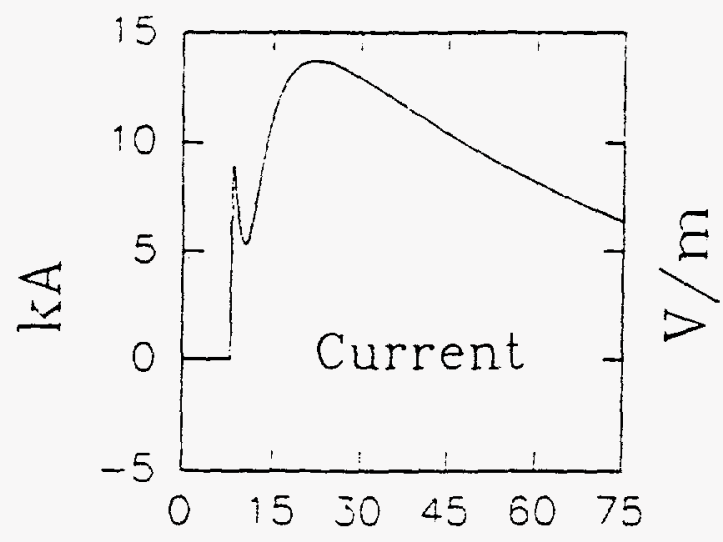

microseconds

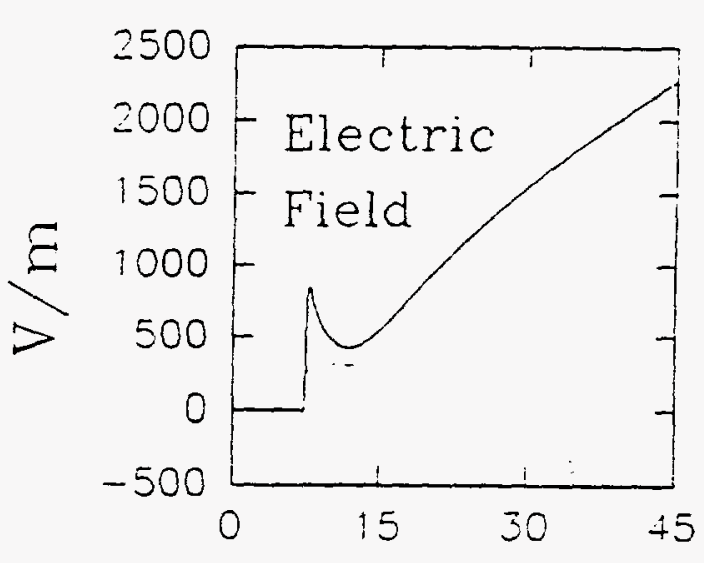

microseconds

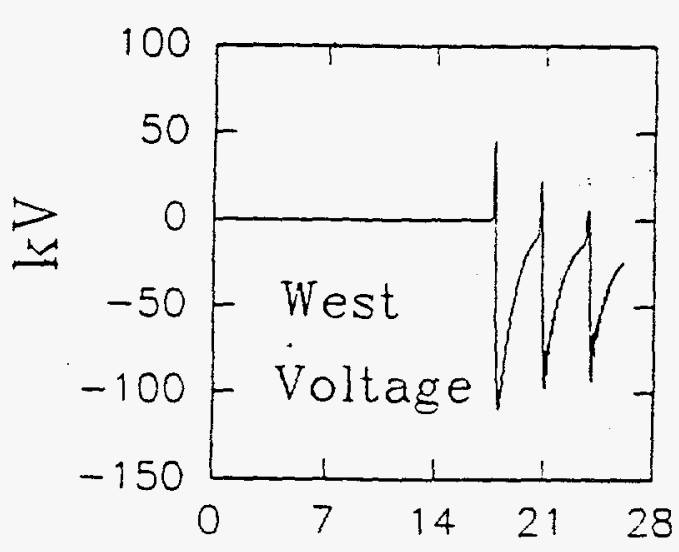

microseconds

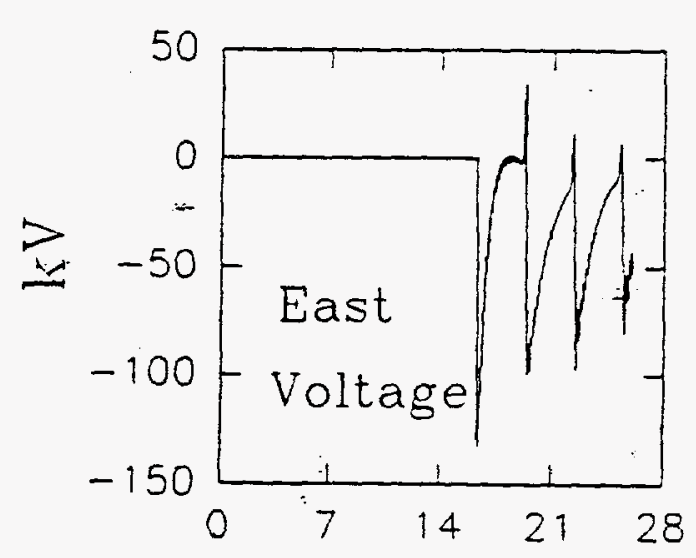

microseconds

Fig. 7.2 Channel-base current (upper left), calculated oscillatory voltages at the east (lower right) and west (lower left) ends of the line, and vertical electric field intensity (upper right) calculated to simulate the second stroke of flash 1 that occurred on day 232. Experimental data are found in Figure $3.5 \mathrm{~b}$. 


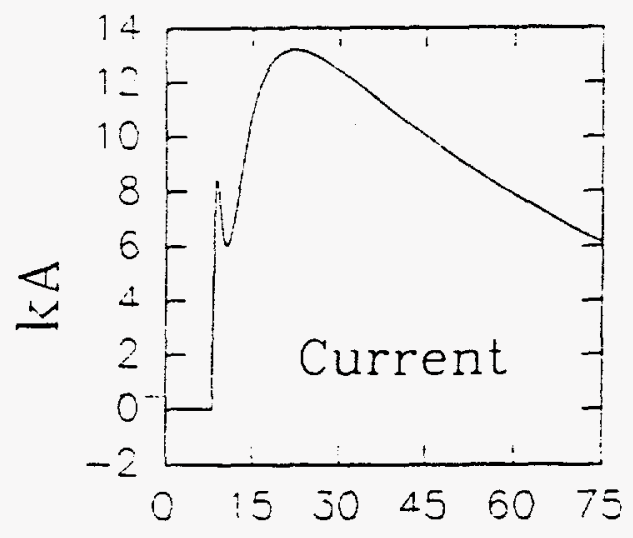

microseconds

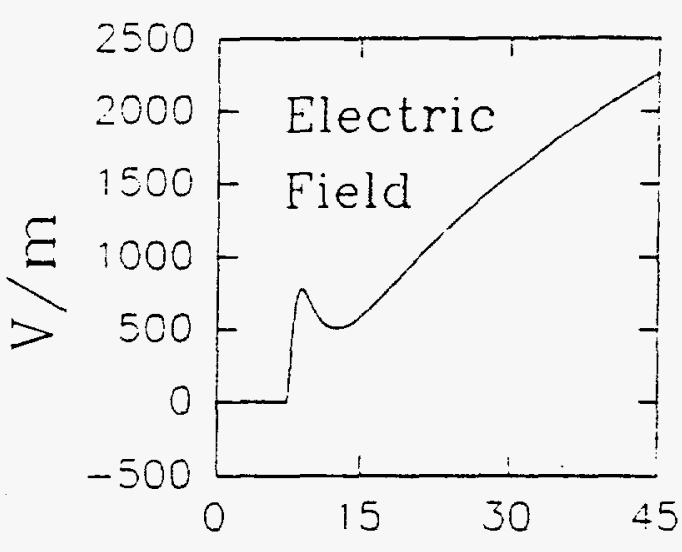

microseconds

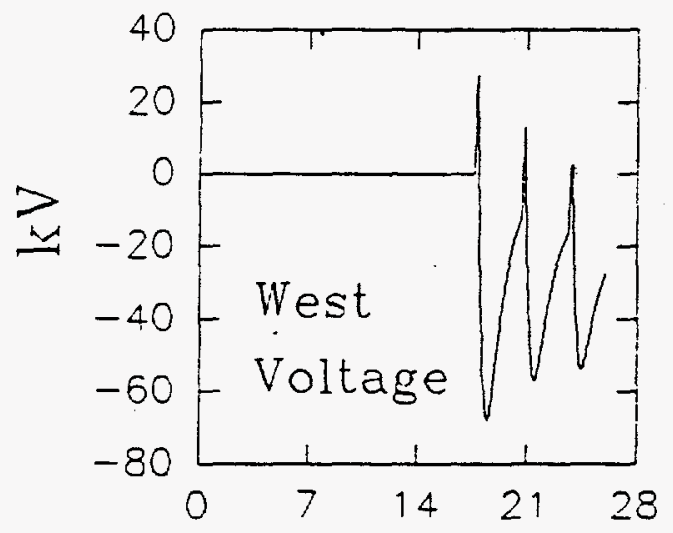

microseconds

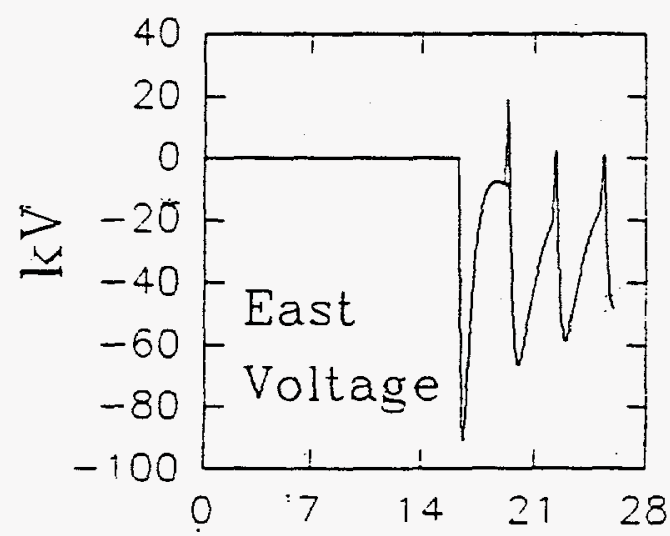

microseconds

Fig. 7.3 Channel-base current (upper left), calculated oscillatory voltages at the east (lower right) and west (lower left) ends of the line, and vertical electric field intensity (upper right) calculated to simulate the sixth stroke of flash 1 that occurred on day 232. Experimental data are found in Figure $3.5 f$. 


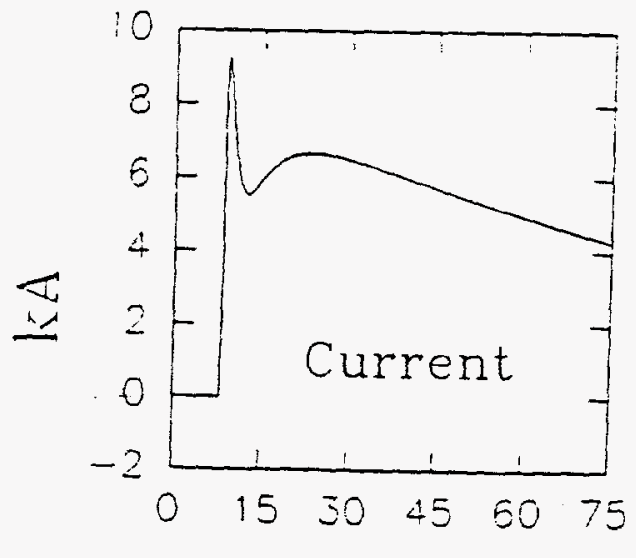

microseconds

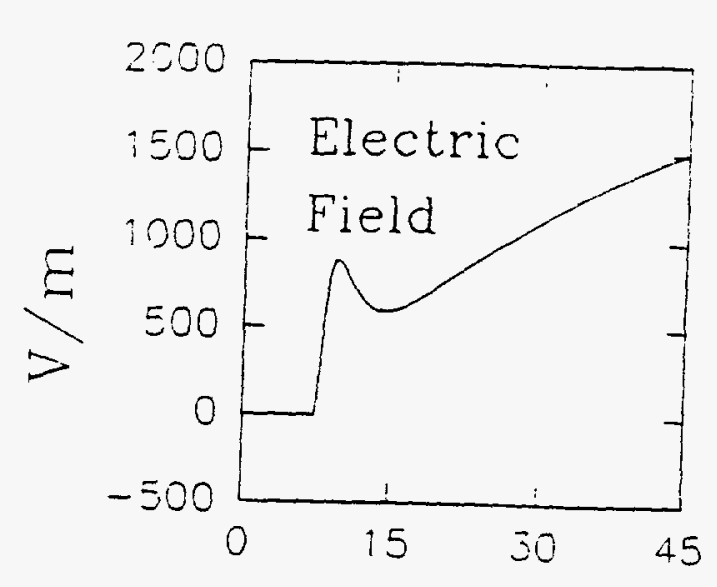

microseconds

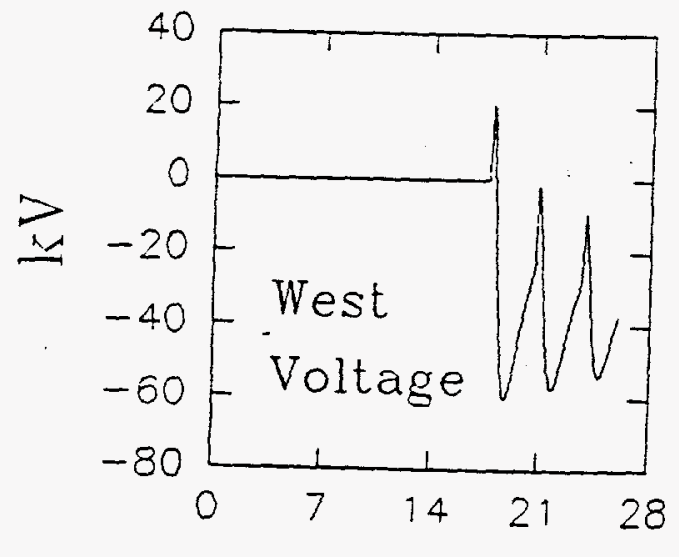

microseconds

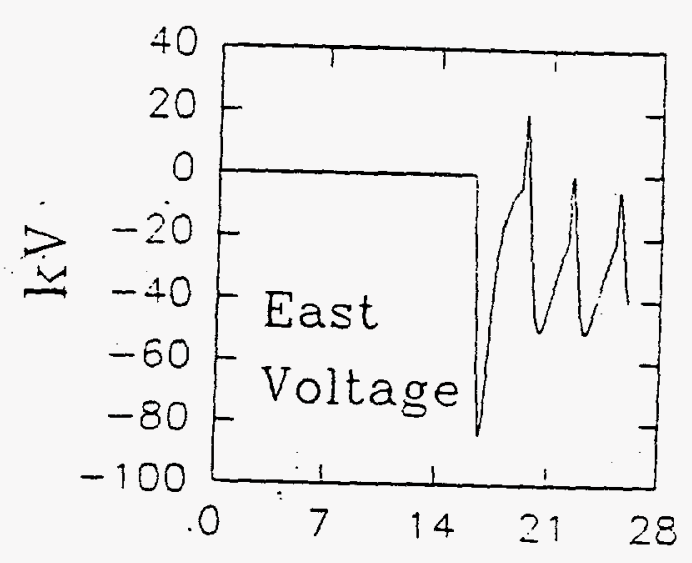

microseconds

Fig. 7.4 Channel-base current (upper left), calculated oscillatory voltages at the east (lower right) and west (lower left) ends of the line, and vertical electric field intensity (upper right) calculated to simulate the first stroke of flash 1 that occurred on day 240 . Experimental data are found in Figure $3.6 \mathrm{a}$. 


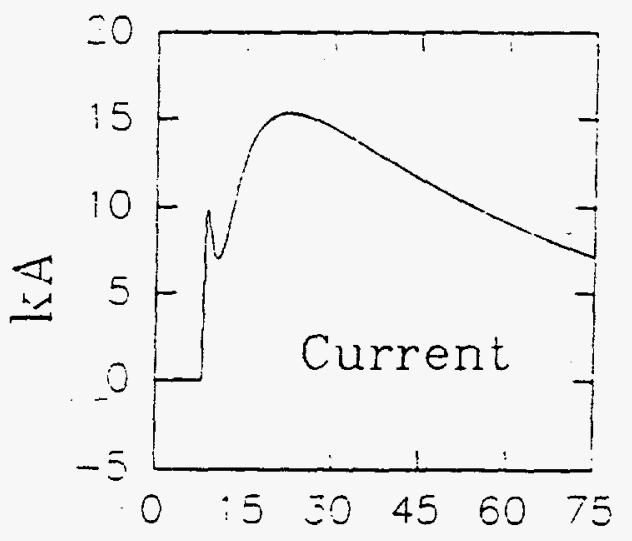

microseconds

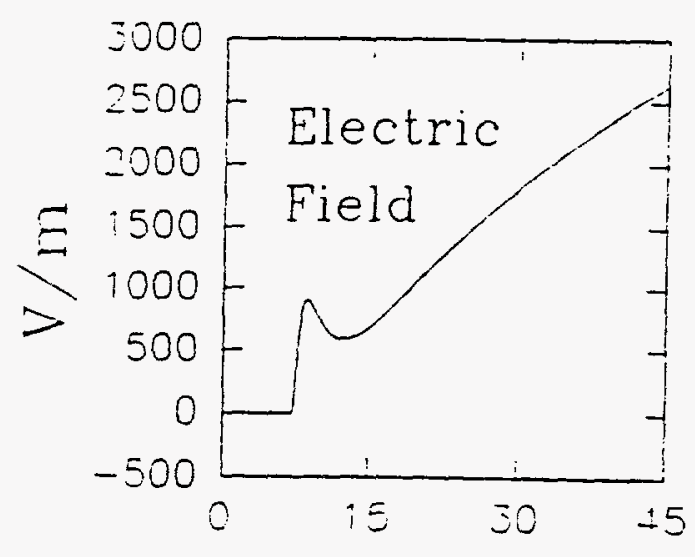

microseconds

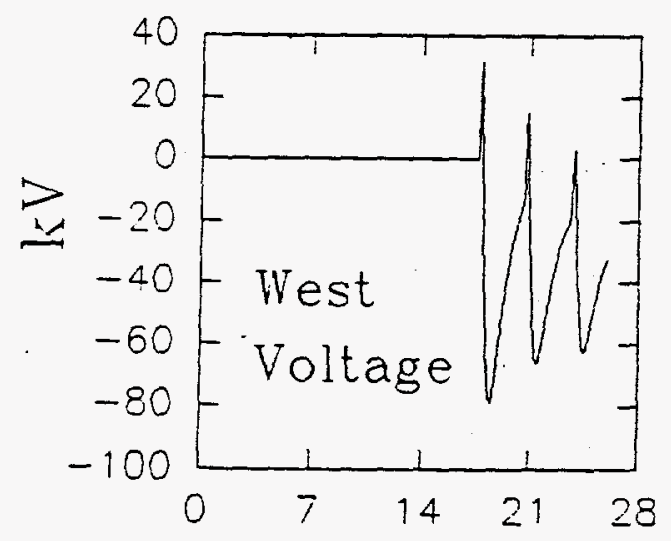

microseconds

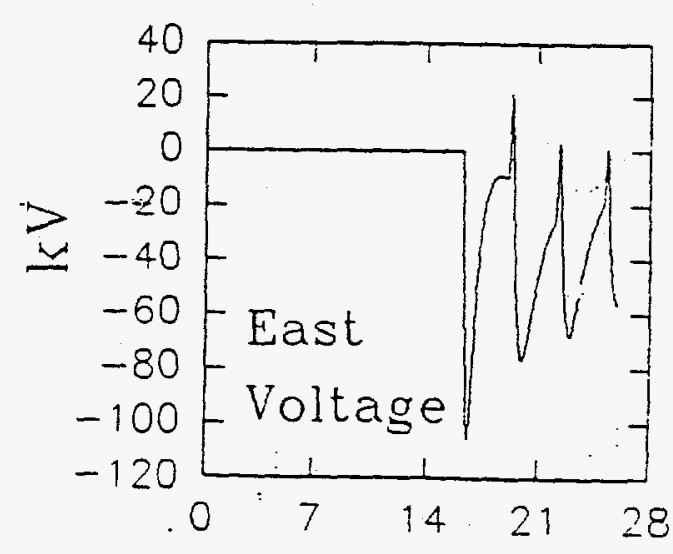

microseconds

Fig. 7.5 Channel-base current (upper left), calculated oscillatory voltages at the east (lower right) and west (lower left) ends of the line, and vertical electric field intensity (upper right) calculated to simulate the second stroke of flash 1 that occurred on day 240 . Experimental data are found in Figure $3.6 \mathrm{~b}$. 


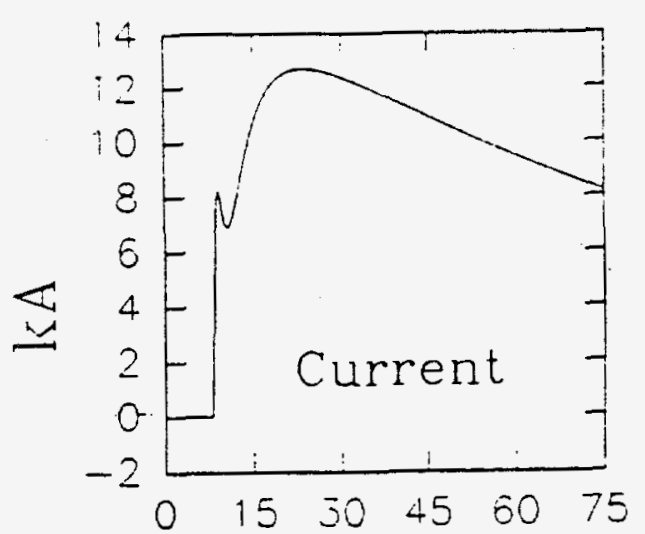

microseconds

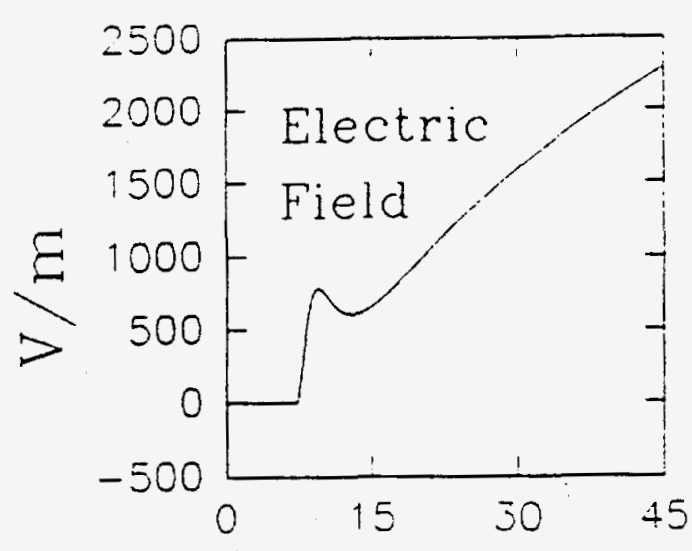

microseconds

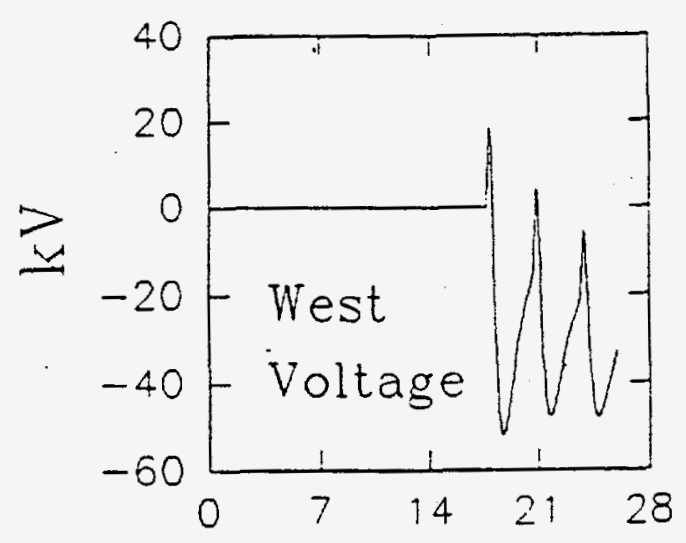

microseconds

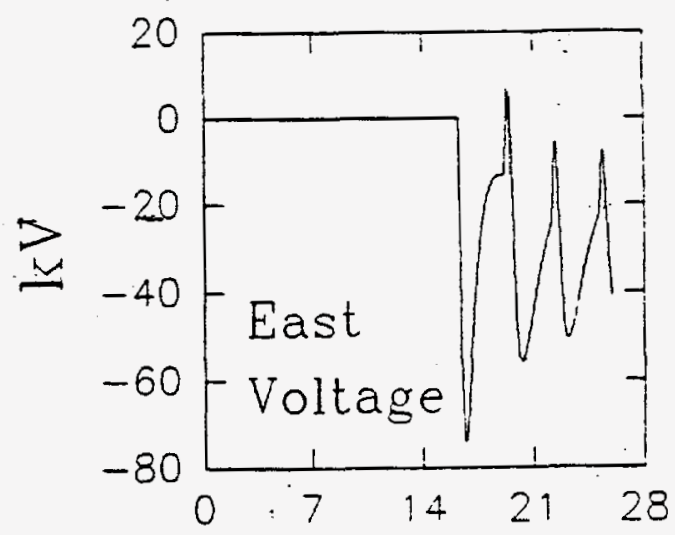

microseconds

Fig. 7.6 Channel-base current (upper left), calculated oscillatory voltages at the east (lower right) and west (lower left) ends of the line, and vertical electric field intensity (upper right) calculated to simulate the first stroke of flash 2 that occurred on day 240. Experimental data are found in Figure 3.7a. 


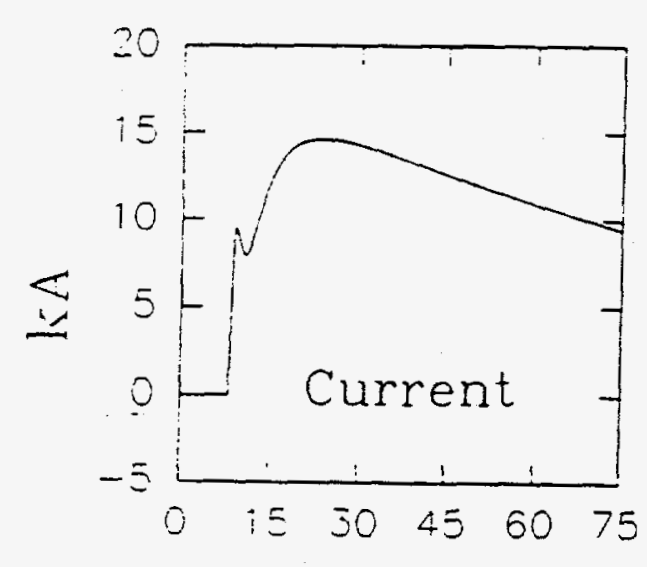

microseconds

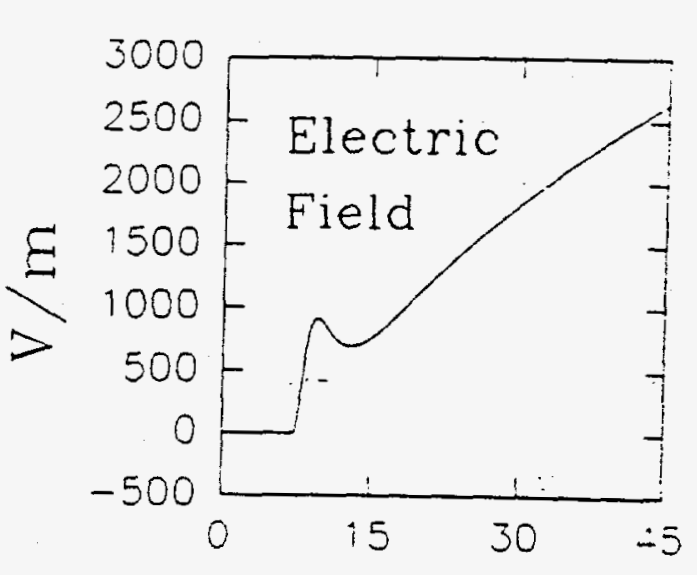

microseconds

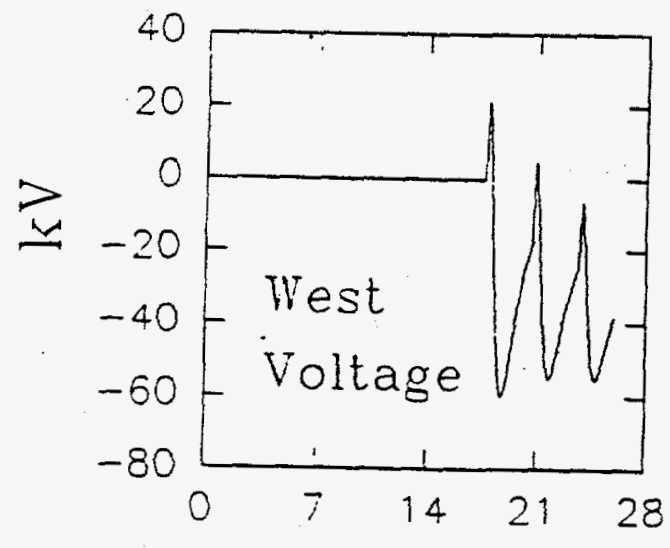

microseconds

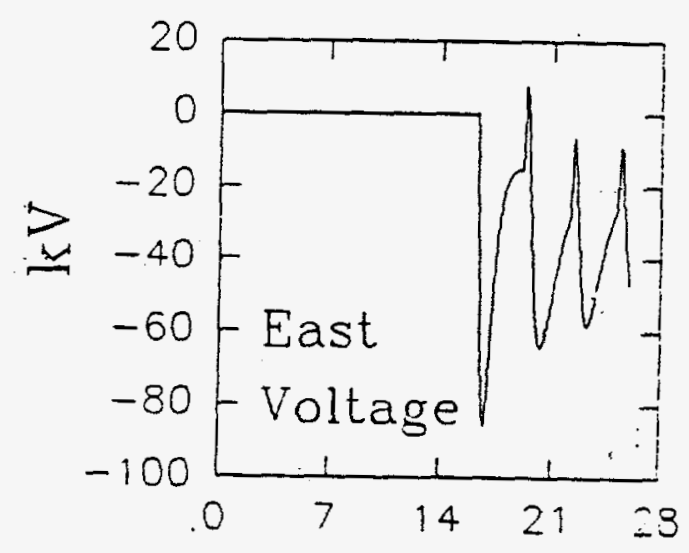

microseconds

Fig. 7.7 Channel-base current (upper left), calculated oscillatory voltages at the east (lower right) and west (lower left) ends of the line, and vertical electric field intensity (upper right) calculated to simulate the second stroke of flash 2 that occurred on day 240. Experimental data are found in Figure $3.7 \mathrm{~b}$. 
This feature, which did not appear in the oscillatory voltages measured on day 240, could not be reproduced in our numerical calculations. This discrepancy might be attributable to an atypical channel geometry such as a significant inclination of the rocket ground wire which could affect the relative amplitudes of the horizontal and vertical fields in the vicinity of the lightning.

The channel-base currents shown in Figures 7.2 through 7.7 have rise times ranging from $200 \mathrm{nsec}$ to $600 \mathrm{nsec}$ and decay time constants ranging from $60 \mu \mathrm{sec}$ to $140 \mathrm{\mu sec}$. The peak amplitudes of the currents needed to simulate the oscillatory voltages range from $9 \mathrm{kA}$ to $15 \mathrm{kA}$. The ranges of parameters of the channel-base currents required to simulate the oscillatory voltages are seen to be consistent with those previously reported in the literature. Although (as mentioned in Section 7.2) we have reason to believe that the current wave shapes measured during the 1986 experiment are in error, it is interesting to note that the peak reported amplitudes for the two two-stroke flashes that occurred on day 240 (both of which contained only oscillatory voltages) range from $12 \mathrm{kA}$ to $16.5 \mathrm{kA}$. For the seven-stroke flash occurred on day 232 , on the other hand, only the peak amplitudes of the return stroke currents associated with the five impulsive voltages, ranging from $25 \mathrm{kA}$ to $55 \mathrm{kA}$ (considerably higher than the currents for day 240) were reported. The peak amplitudes of the remaining two return stroke currents (associated with oscillatory voltages) did not trigger the system which had a lower measurement threshold of $6 \mathrm{kA}$.

We now look at the oscillatory voltages in more detail. Even though both the theoretical voltages and the measured oscillatory voltages exhibit a characteristic sequence of negative pulses, the following discrepancies exist: 
(1) The decay in the amplitudes of the negative pulse series in the theoretical voltage waveforms is slower than the decay observed in the measured waveforms.

(2) The time between successive pulses is longer for the measured than for the calculated waveforms.

(3) The positive change observed to precede the first negative pulse in the measured voltages at both ends of the line is absent in the calculated east voltage waveforms, and it emerges too abruptly in the voltage waveforms at the west end.

The first two discrepancies described above are consistent with the existence of corona effects on the line that have not been taken into account. Corona effects produce an increase in the energy losses as the waves travel along the line making them decay faster. Corona effects also increase the capacitance per unit length of the overhead wires thereby reducing the wave propagation speed as does the increase in resisitance due to corona losses. Al-Tai et al. (1989) have successfully simulated corona effects by introducing into the transmission line model a linear resistance per unit length and a capacitance per unit length that could be assumed to be either constant, in which case Al-Tai et al. (1989) obtained fair results compared to published measurements of voltages affected by corona, or nonlinear, for which the results improved although the implementation becomes considerably more difficult. Based on their results, we have modelled corona effects by increasing the capacitance and the resistance per unit length in our computer code with improved, agreement, as shown in Figures 7.8 through 7.13 . 

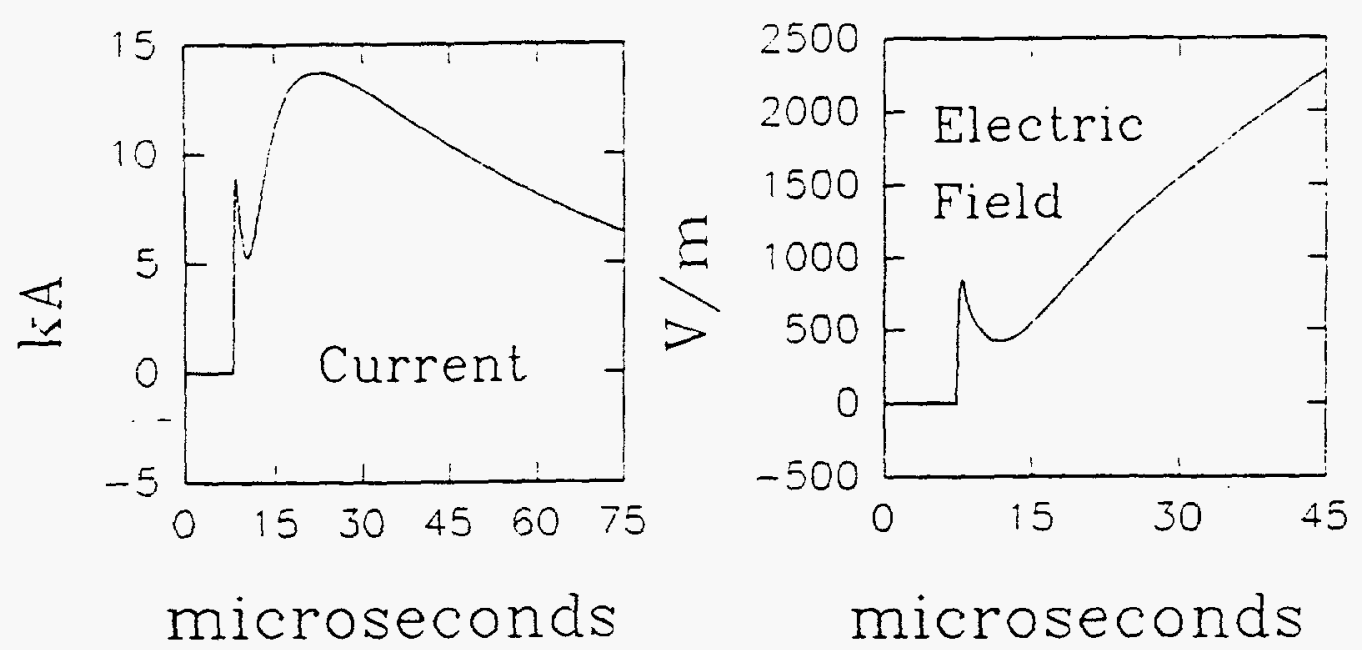

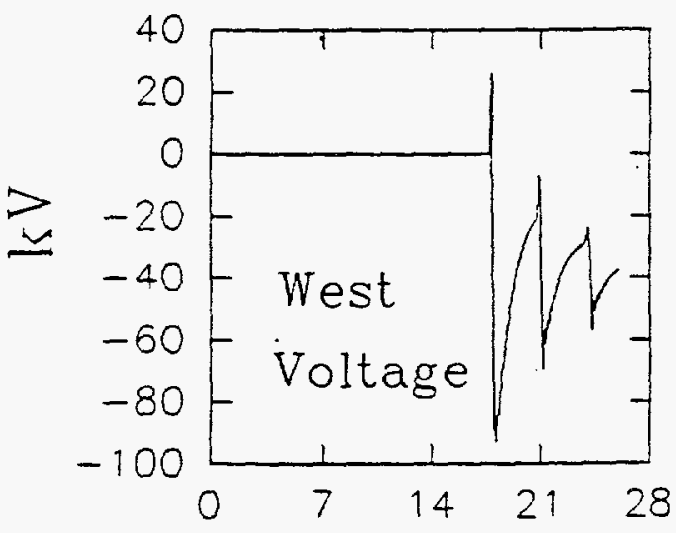

microseconds

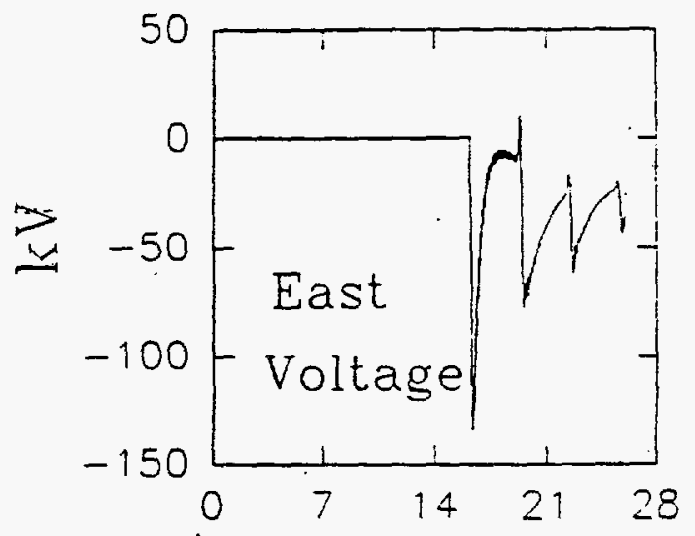

microseconds

Fig. 7.8 Simulation of corona effects. The capacitance matrix of the line was changed to account for the increase in the radius of the wires due to corona and the resistance per unit length was set to $1 \mathrm{ohm} / \mathrm{m}$ to model the losses due to corona. The time between successive reflected pulses is 3.1 usec. Experimental data are shown in Figure $3.5 b$. 


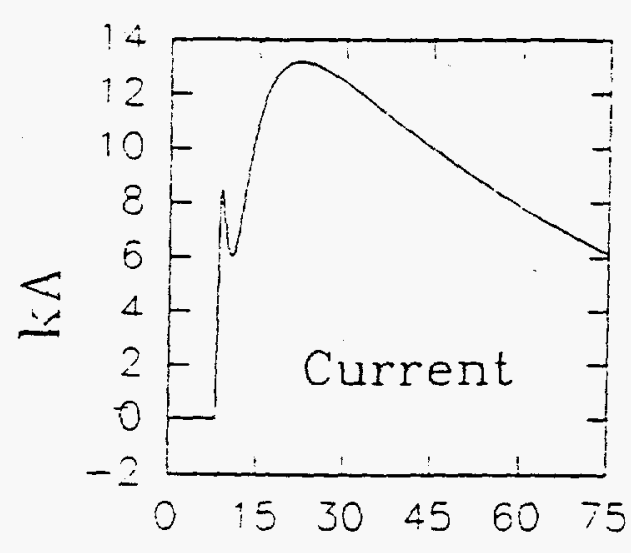

microseconds

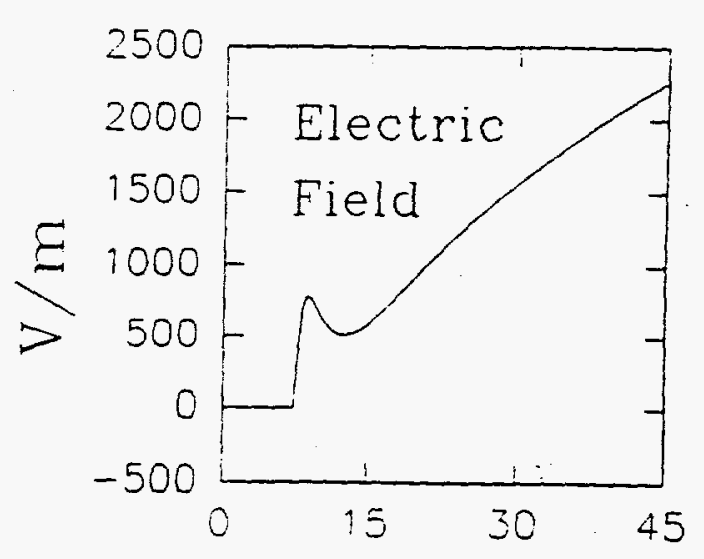

microseconds

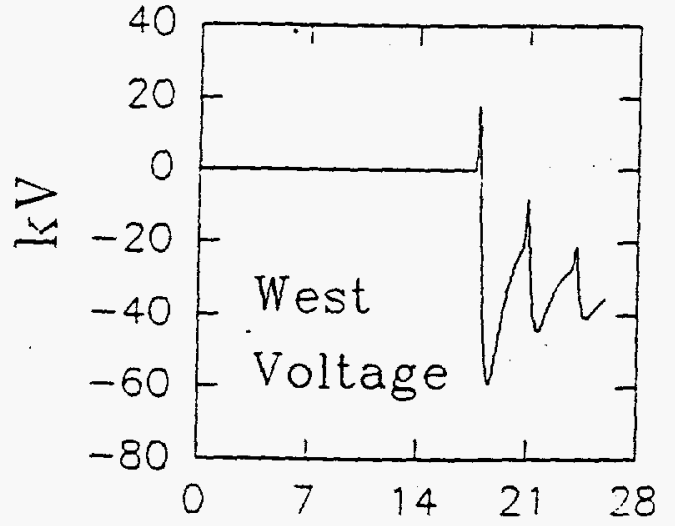

microseconds

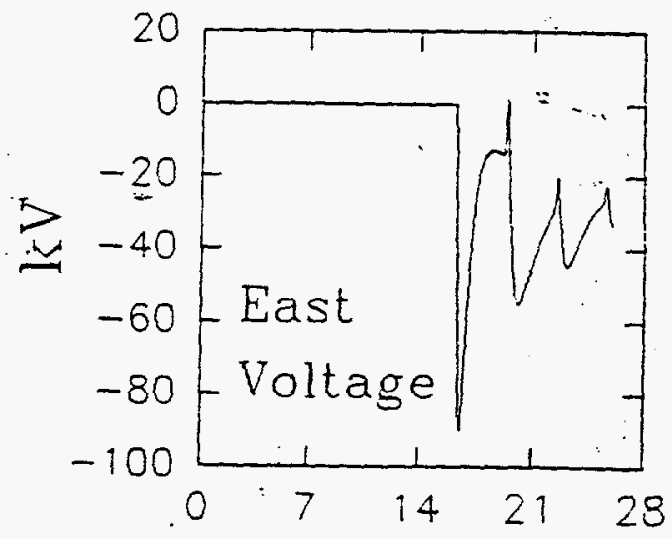

microseconds

Fig. 7.9 Simulation of corona effects. The capacitance matrix of the line was changed to account for the increase in the radius of the wires due to corona and the resistance per unit length was set to $1 \mathrm{ohm} / \mathrm{m}$ to model the losses due to corona. The time between successive reflected pulses is 3.1 ksec. Experimental data are shown in Figure $3.5 \mathrm{f}$. 


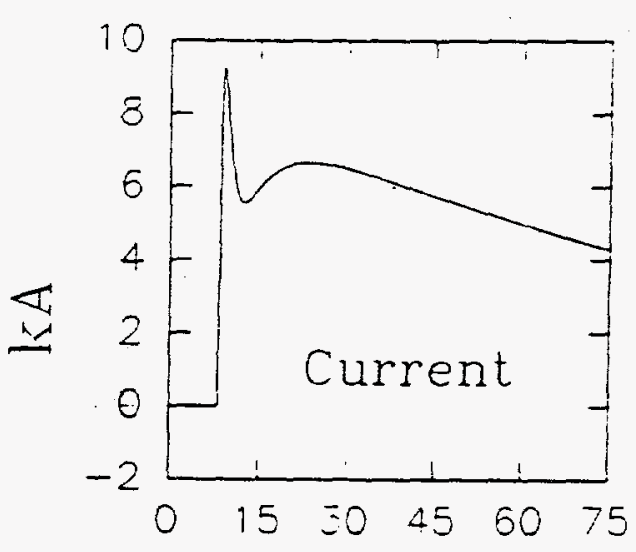

microseconds

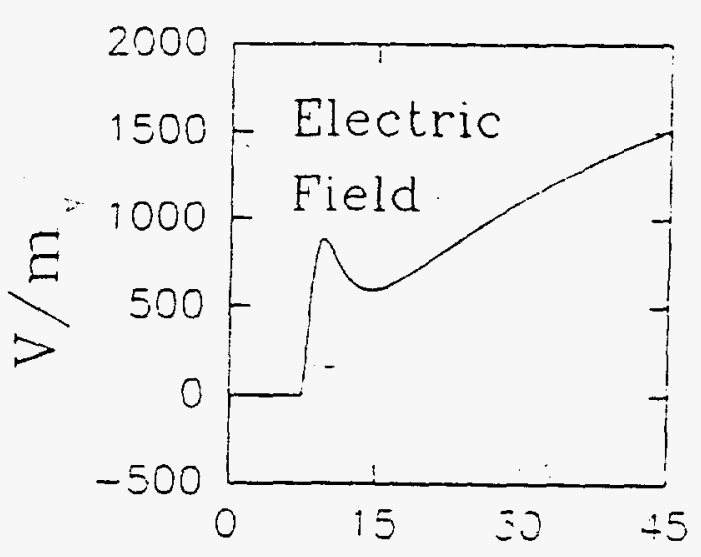

microseconds

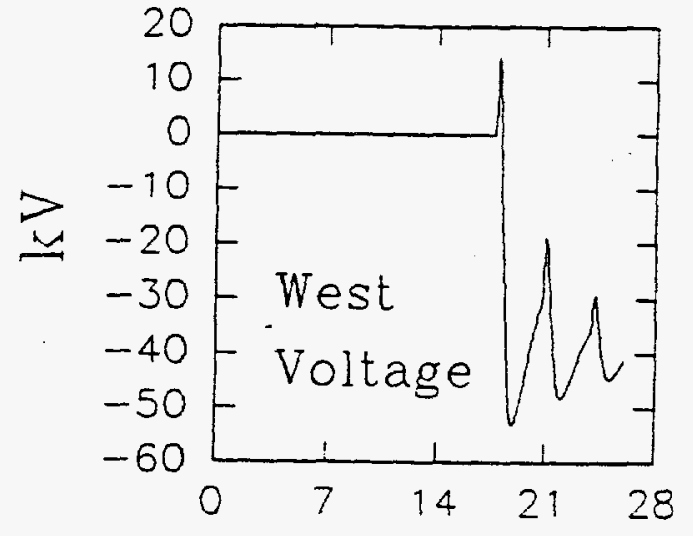

microseconds

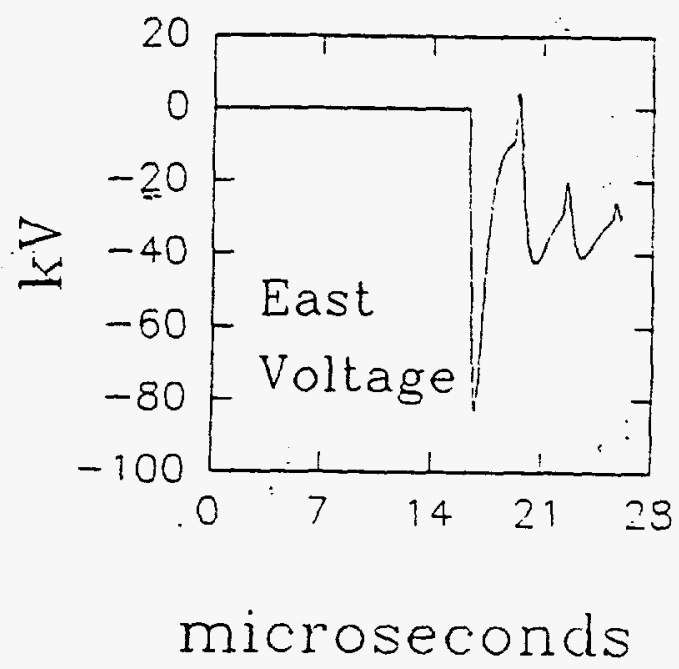

Fig. 7.10 Simulation of corona effects. The capacitance matrix of the line was changed to account for the increase in the radius of the wires due to corona and the resistance per unit length was set to $1 \mathrm{Ohm} / \mathrm{m}$ to model the losses due to corona. The time between successive reflected pulses is $3.1 \mu \mathrm{sec}$. Experimental data are shown in Figure $3.6 \mathrm{a}$. 


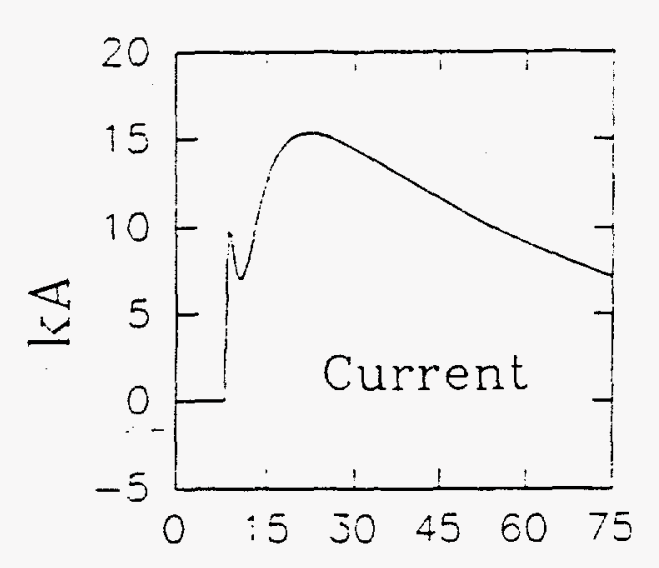

microseconds

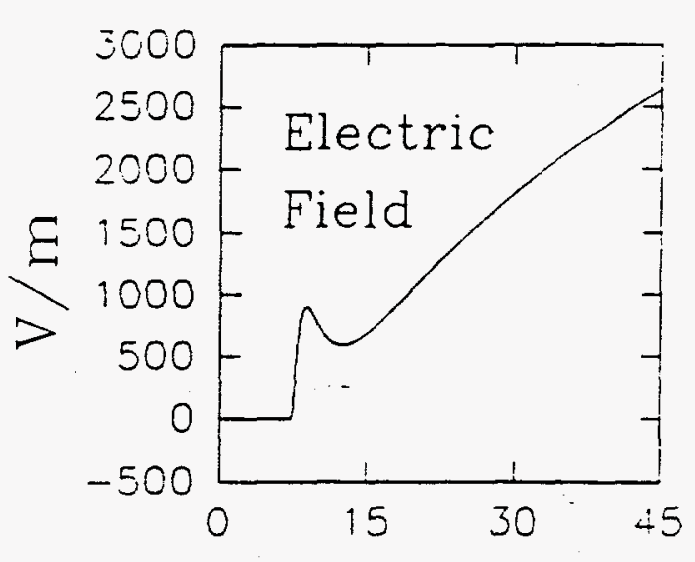

microseconds

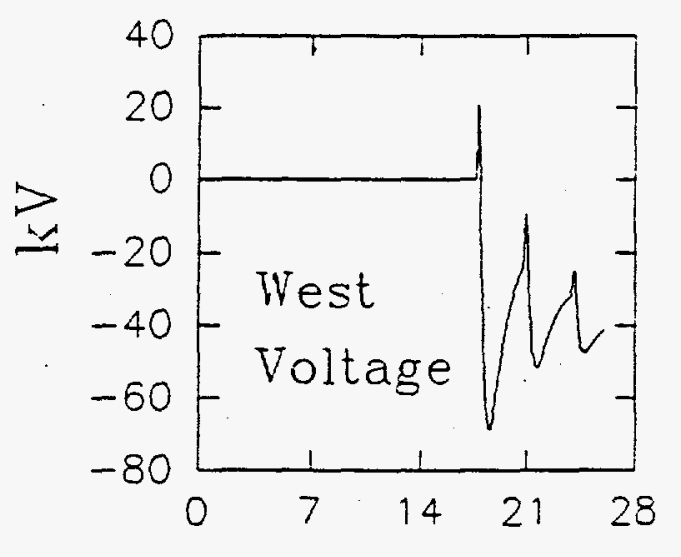

microseconds

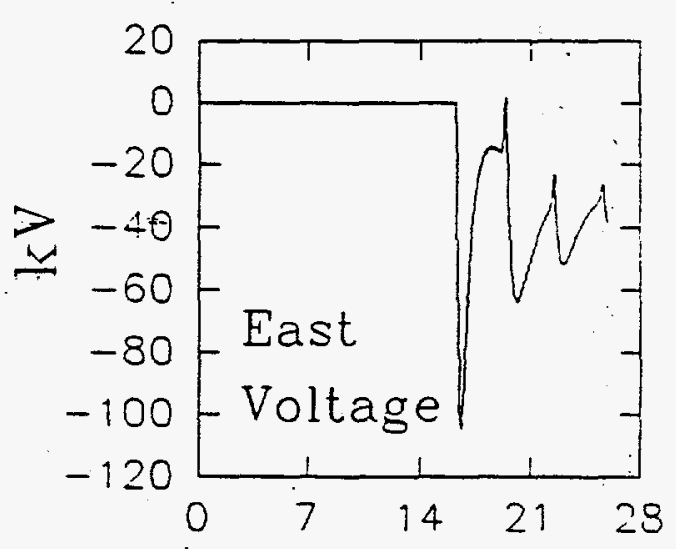

microseconds

Fig. 7.11 Simulation of corona effects. The capacitance matrix of the line was changed to account for the increase in the radius of the wires due to corona and the resistance per unit length was set to $1 \mathrm{Ohm} / \mathrm{m}$ to model the losses due to corona. The time between successive reflected pulses is 3.1 usec. Experimental data are shown in Figure $3.6 \mathrm{~b}$. 


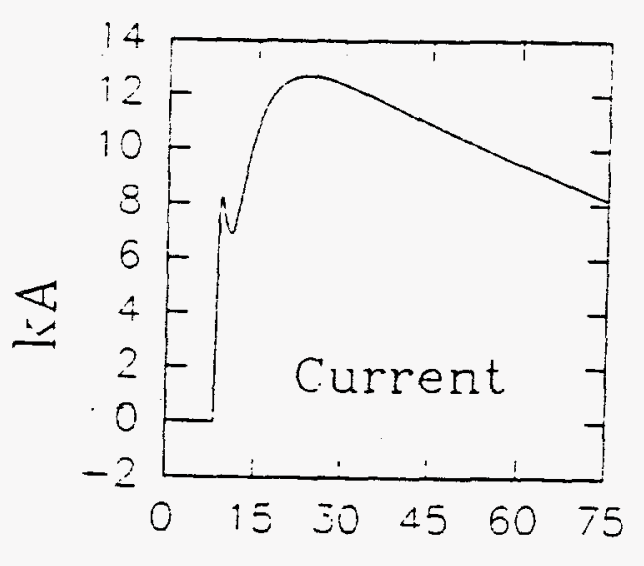

microseconds

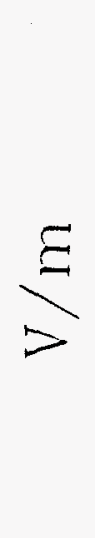

500

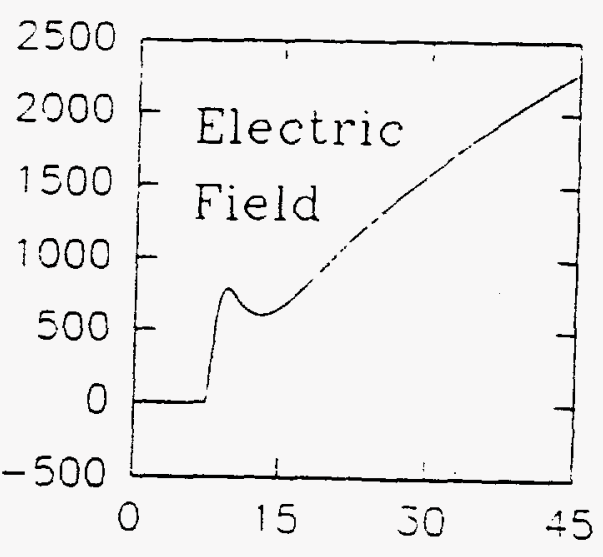

microseconds

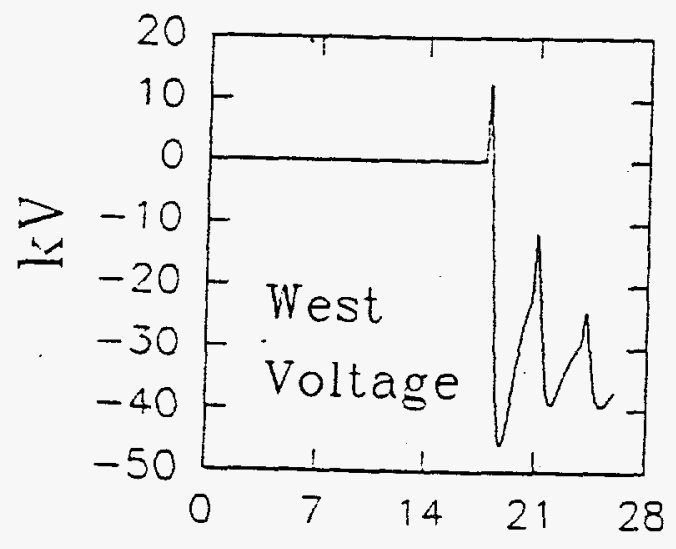

microseconds

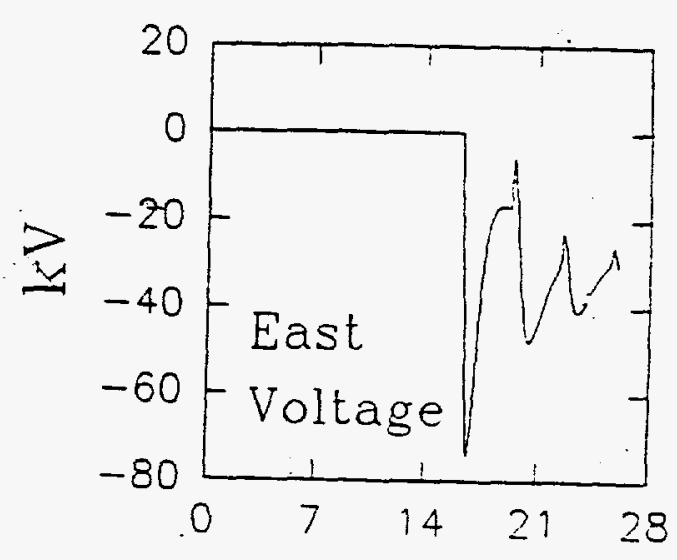

microseconds

Fig. 7.12 Simulation of corona effects. The capacitance matrix of the Iine was changed to account for the increase in the radius of the wires due to corona and the resistance per unit length was set to $1 \mathrm{ohm} / \mathrm{m}$ to model the losses due to corona. The time between successive reflected pulses is $3.1 \mathrm{\mu sec}$. Experimental data are shown in Figure $3.7 a$. 


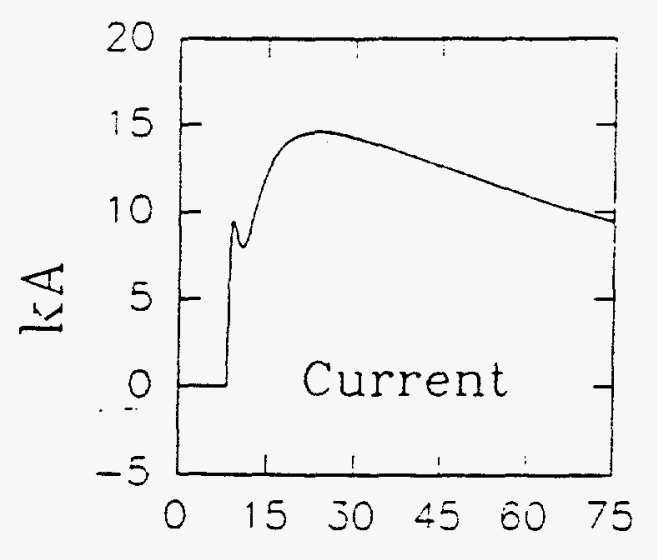

microseconds

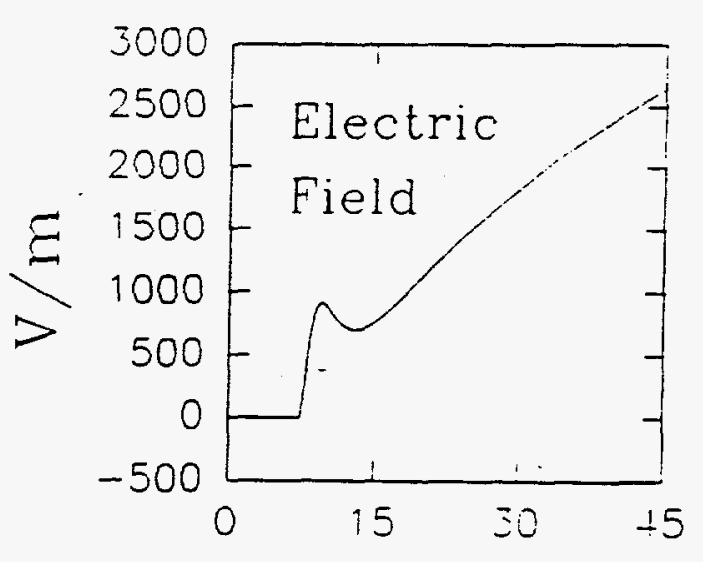

microseconds

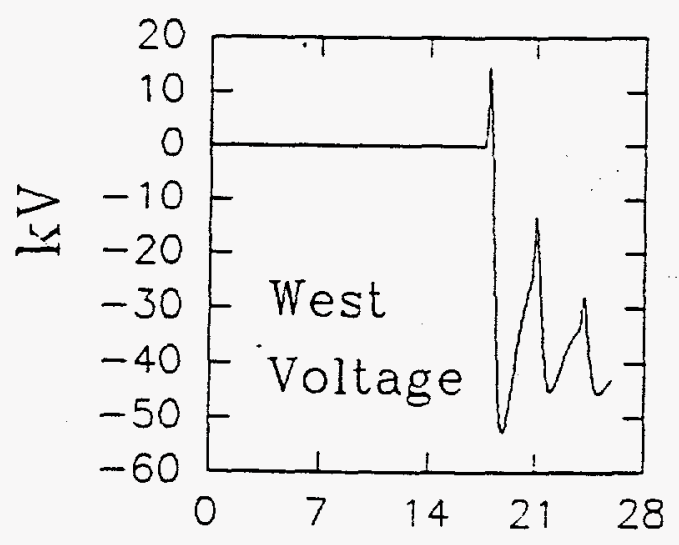

microseconds

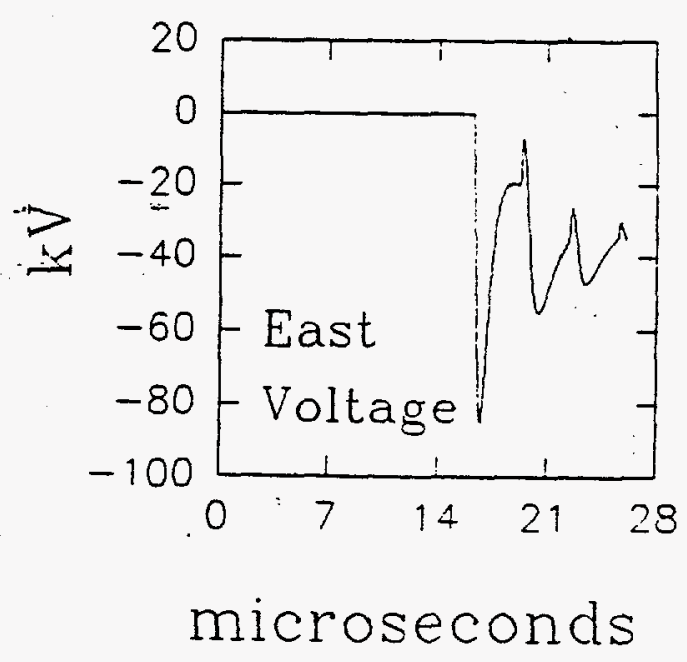

Fig. 7.13 Simulation of corona effects. The capacitance matrix of the line was changed to account for the increase in the radius of the wires due to corona and the resistance per unit length was set to $1 \mathrm{ohm} / \mathrm{m}$ to model the losses due to corona. The time between successive reflected pulses is 3.1 msec. Experimental data are shown in Figure $3.7 \mathrm{~b}$. 
We used the value of the corona capacitance corresponding to $100 \mathrm{kVolts}$, the order of magnitude of the measured oscillatory voltages, and an arbitrary value of $1 \mathrm{Ohm} / \mathrm{m}$ for the resistance per unit length. This change caused the time between successive reflections to increase from 3.0 to $3.1 \mu \mathrm{sec}$, becoming closer to the measured value, $3.3 \pm 0.1 \mu \mathrm{sec}$, and the amplitude decay of successive reflections to agree better with that observed in the measured waveforms. The third discrepancy described above, that of the absence of a relatively slow positive change in the calculated voltage waveforms at the east and west ends of the line, is likely to be due to coupling effects from the leader preceding the return stroke, which we have not modelled here.

We now turn our attention to the impulsive voltages characterized in Chapter III. It was pointed out in Chapter III that the vertical electric fields associated with impulsive voltages are about two and a half times larger than those associated with oscillatory voltages and that the voltage amplitudes measured at the east and west ends are, in turn, five to ten times larger for the impulsive voltages than for the oscillatory voltages, with the impulsive voltages being measured at several hundred kVolts at the east end to 1 MVolt at the west end. High voltage levels and the physical arrangement of the resistive terminations and the voltage dividers (shown in Figure 7.14) suggest the possibility of flashovers. This is supported by the small spread (the standard deviation of the peak amplitudes being about $10 \%$ of the average east and west end voltages) of the peak amplitudes in the measured impulsive voltages at either end of the line.

Visual observations, which would have allowed us to determine unequivocally if and where flashovers occurred, were not made. In what follows, we list and discuss the two flashover cases most likely to have 


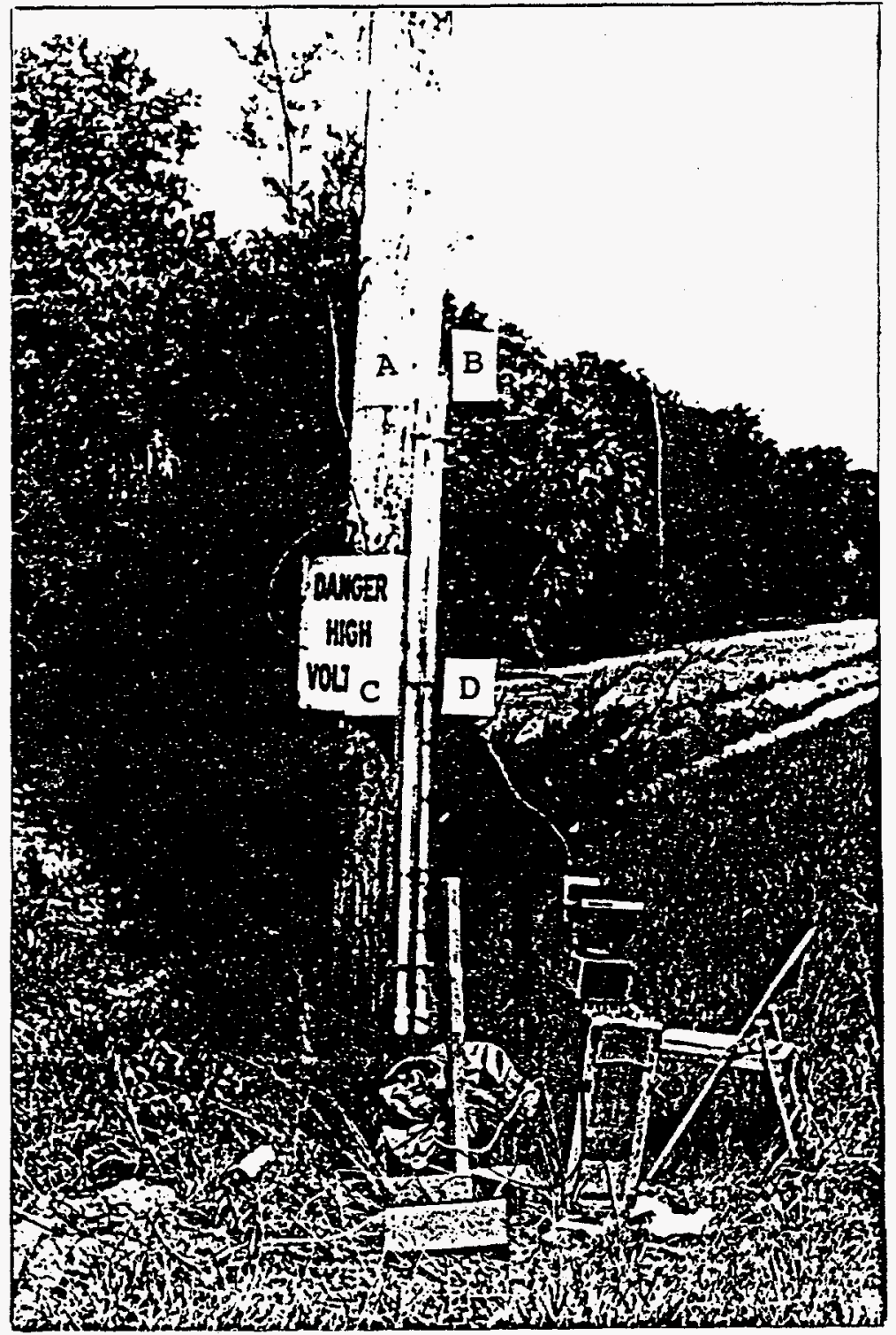

Fig. 7.14 A photograph showing the physical arrangement of the resistive terminations and the voltage dividers. Points $A$ and $B$ are the top screw terminals, and points $C$ and $D$ are the center clamps. 
occurred, and then we simulate them with our time domain computer program. It should be noted that the resistive terminators and voltage dividers were inspected for evidence of flashovers, such as charring, but that no such evidence was observed.

Type a.- Flashovers were likely between the top screw terminals (identified as $A$ and $B$ in Figure 7,14). The distance between the top screw terminals has been estimated from photographs at about $6 \mathrm{~cm}$ for both the east and the west ends. No direct distance measurements were made during the experiment because their importance was not realized. The effect of a flashover between $A$ and $B$ would be to change the terminating resistance from $10.8 \mathrm{kOhm}$ to the shunt combination of 10.8 kOhm and $1.08 \mathrm{kOhm}$, or about $980 \mathrm{ohm}$, leaving the voltage divider ratio unchanged.

Type b.- Flashovers were also possible between the center clamps (identified as $C$ and $D$ in Figure 7.14). The voltage between $C$ and $D$ reached as high as half the line voltage since $C$ is a center tap of the voltage divider and $D$ is at ground potential as long as no current flows through the 1 kohm resistor. The minimum distance between $C$ and $D$ was again estimated from the available photographs to be $4 \mathrm{~cm}$ for the east end and $2.5 \mathrm{~cm}$ at the west end. No direct measurements were made of these distances while the experiment was being performed because it was not recognized that they were important. In the case of a flashover between clamps $C$ and $D$, the terminating resistance would change to half of $10.8 \mathrm{kOhm}$ in series with the parallel combination of $530 \mathrm{Ohm}$ and 5.4 kOhm. This translates into a $5.9 \mathrm{kOhm}$ resistor. The voltage divider ratio, on the other hand, would change to be 5.9 times smaller than under no flashover conditions. 
We have simulated the four possible combinations of the above flashover cases for different current waveforms in order to obtain a match between the measured impulsive voltages and those calculated numerically. Wave shape agreement was obtained only for the case where type $b$ flashovers are assumed at each end of the line. Physically, type b flashovers are more likely to occur since they involve electrodes that are in contact with the wet wooden poles, and the breakdown voltage between two electrodes that are in contact with a common unpolished surface is generally smaller than the breakdown voltage between two electrodes at the same distance, but separated by air.

Only one simulation will be shown since all the measured impulsive voltages are essentially identical. The current waveform that yielded the best amplitude and wave shape agreement for the fifth stroke of flash 1 on day 232 is shown in Figure 7.15. In Figures 7.16 we have plotted the corresponding calculated vertical electric field intensity at $500 \mathrm{~m}$ as well as the voltages at either end of the line for the only flashover combination that yields a reasonable match when compared with the experimental data. The following similarities and differences between these calculated impulsive voltages and their measured counterparts can be observed: (1) Both the measured and the calculated voltages at either end of the line exhibit a main negative pulse which determines the peak amplitude of the waveforms. (2) Only in the west end measured voltages is the main negative pulse followed by a series of small discernible pulses at about $3 \mathrm{\mu sec}$ intervals, each having two positive-going peaks, whereas the calculated voltages exhibit such pulses at both ends of the line. We have not been able to explain the absence of these pulses in the measured voltages at the east end since, if the pulses are reflections traveling to and from the east and west ends, they should be present at 


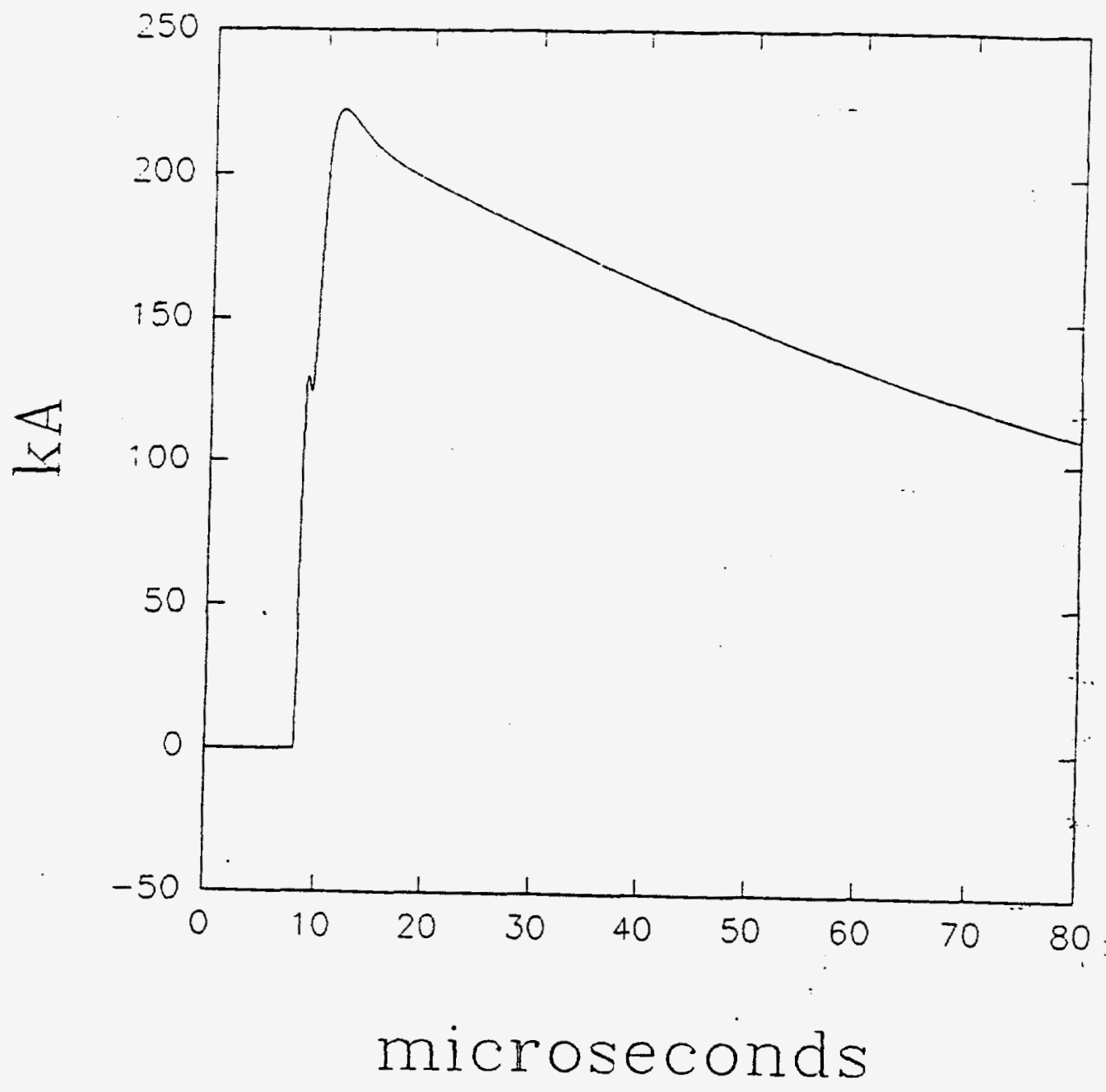

Fig. 7.15 Current used for simulation of the electric field intensity and voltages produced by the fifth stroke in flash 1 occurred on day 232 . 


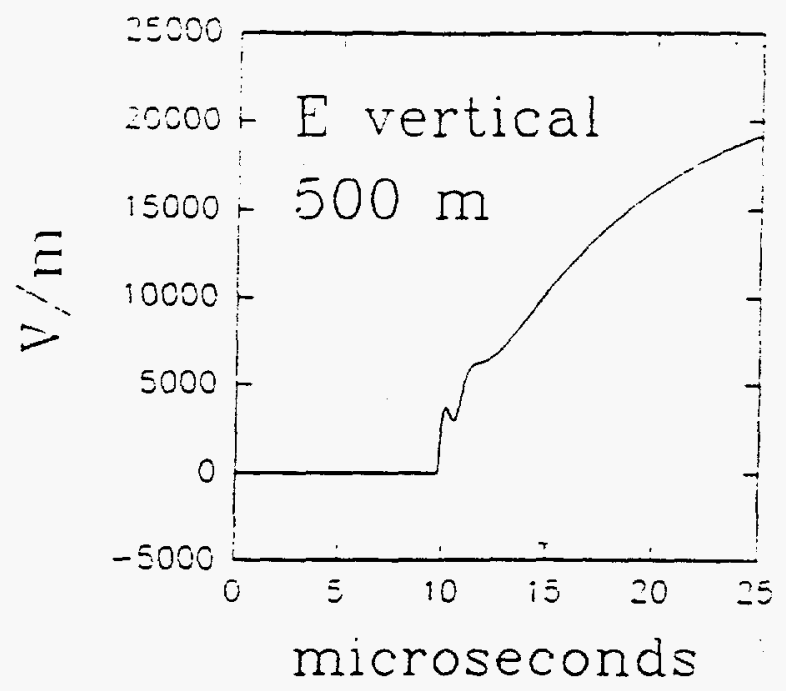

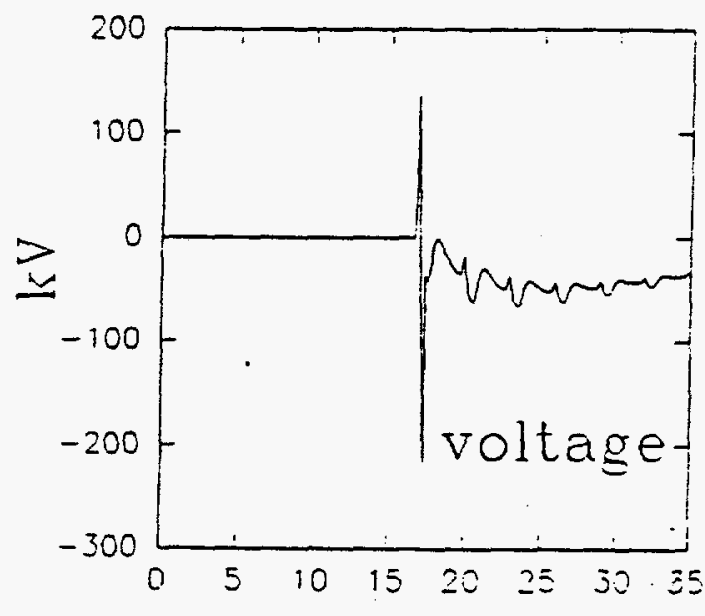

- microseconds

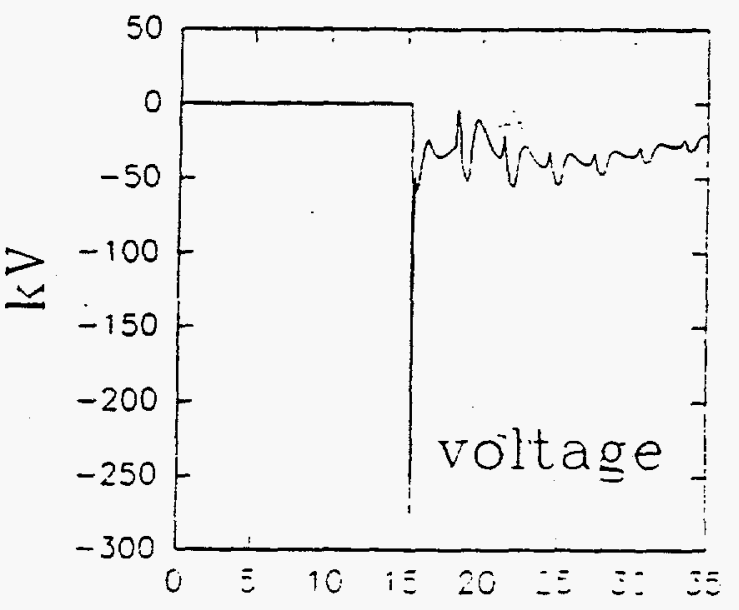

microseconds

Fig. 7.16 vertical electric field intensity at $500 \mathrm{~m}$ and voltages at either end of the test line calculated by simulating flashovers (defined in the text) at both ends of the line. The flashover voltages at each end were chosen to obtain amplitudes consistent with those in the fifth stroke in flash 1 occurred on day 232 (see Figure $3.5 e$ ). 
the both ends with similar amplitudes. (3) The positive-going pulse observed at the start of the voltages observed in the measured voltage wave forms at the east and west ends of the line appears only in the calculated voltages at the west end of the line. Again, as in the case of the oscillatory voltages, this is likely to be due to coupling effects from the leader, not modelled here. A channel-base current with a peak amplitude of $220 \mathrm{kA}$, much higher than the maximum amplitude of $60 \mathrm{kA}$ reported by Leteinturier et al. (1988a,b, 1990) for rocket triggered lightning was necessary in order to obtain good amplitude agreement. Thus there is clearly a problem in the modeling of the impulsive voltages. Possibly we have not properly understood the several flashover possibilities.

Although, as mentioned earlier in this chapter, the channel-base currents used in the theoretical simulations have rise and decay times within the ranges reported in the literature, in should be pointed out that important discrepancies exist between the wave shapes of the theoretical currents used in this chapter and those reported in the literature: the sequence of a sharp peak at the start of the theoretical currents, followed by a broad hump which exhibits its peak typically about $15 \mu \mathrm{sec}$ after the start of the current (see for example Figures 7.2 through 7.7 ), does not appear in the measured currents. Instead, the measured currents are characterized by either the absence of the hump portion or the absence of the initial peak. When the peak is absent, a change of slope in the rise to peak takes its place, with the ensuing hump exhibiting its peak at times of the order of a one or two microseconds after the onset of the current.

We have seen how the coupling model of Agrawal et al. (1980), the Diendorfer-Uman return stroke model, and the field calculation techniques 
developed in Chapter IV can be used to calculate line voltages from very close lightning that are in large part consistent with experimental measurements, although some inconsistencies remain in the modeling of non-linear flashover effects and in the modeling of the amplitudes of impulsive voltages which may or may not be due to non-linear effects. 



\section{CHAPTER VIII}

\section{OVERHEAD CLOUD IIGHTNING: THEORY VERSUS EXPERIMENT}

\subsection{Coupling Theory For Overhead Lightning}

\subsubsection{Frequency-Domain Coupling Equations}

The frequency-domain transmission line (TL) equations are given here for the top-phase of the unenergized distribution line over a ground of finite-conductivity. The basic assumptions underlining the TL model in the frequency domain are similar to those used in the derivation of the time-domain equations in Chapter $v$. Two equivalent formulations are possible when the TI model is used. One formulation results in two differential equations in terms of the total line voltage and induced current with two source terms, and the other formulation results in two differential equations in terms of the scattered line voltage and induced current with one source term (see, for example, Ianoz et al., 1988). The frequency-domain computer code, EMPLIN, uses the second formulation, resulting in Eq. (8.1) and Eq. (8.2):

$$
\frac{\partial V^{s}(x)}{\partial x}+Z I(x)=E_{x}^{\theta}(h, x)
$$

and

$$
\frac{\partial I(x)}{\partial x}+Y V^{s}(x)=0
$$

In Eq. (8.1) and Eq. (8.2), $I(x)$ is the line current, and $V^{2}(x)$ is the scattered voltage, the voltage resulting from $I(x)$; and $Z$ and $y$ are the impedance and admittance of an elemental section of the line which includes the effect of the finite-conductivity ground, $\mathbf{Z}_{g}$ and $\mathbf{Y}_{q}$. Different approximate expressions for $Z_{g}$ and $Y_{g}$ can be found and are given in Ianoz et al. (1988). The only source term, $E_{x}^{e}(h, x)$ in Eq. (8.1), is 
the incident along-the-line component of the horizontal electric field plus its ground reflection at line height, although the vertical component of the electric field does play a role in the coupling to the vertical risers. The total voltage at any point along the line, $V(x)$, is defined as the sum of the excitation and scattered voltages:

$$
V(x)=V^{e}(x)+V^{s}(x)
$$

where,

$$
V^{*}(x)=-\int_{0}^{h} E_{z}^{e}(z, x) d z
$$

At the line terminations, the boundary condition and the termination current, $I_{T}$, and the vertical component of the electric field are used to determine the end voltage. The total voltage, $v(x)$, at the line terminations must equal $I_{T} \cdot z_{T}$ at all times, where $z_{T}$ is the termination impedance.

\subsubsection{EMPLIN}

The version of the EMPLIN computer code used in this study is written in BASIC and it allows the calculation of the voltage and current in the frequency domain at one end of a power line in response to an incident electromagnetic wave. The power line is assumed to be a perfect conductor above a finite-conductivity ground. The line geometry that is involved is shown in Figure 4.2. Inputs to the EMPLIN code that help to define the geometry, electrical properties, and excitation of the line for an incident lightning electromagnetic wave are:

$$
\begin{aligned}
\mathrm{h}= & \text { Height of line over earth (in } \mathrm{m} \text { ), equal to } 10 \mathrm{~m} . \\
\mathrm{L}= & \text { Length of line (in } \mathrm{km} \text { ), equal to } 0.448 \mathrm{~km} . \\
\mathrm{a}= & \text { Radius of conductor forming the line (in } \mathrm{cm} \text { ), equal to } 0.4 \\
& \mathrm{~cm} .
\end{aligned}
$$


$\sigma_{\mathrm{g}} \quad=$ Conductivity of the ground, varied between 0.016 and 0.0025 mho/m.

$e_{g} \quad=$ Relative dielectric constant of the ground, equal to 20 .

$\Phi$ = Angle of incidence above the horizon, different for each event.

$\phi \quad=$ Angle of incidence in the azimuth plane, different for each event.

$\alpha_{h} \quad=$ Horizontal polarization parameter, different for each event. $\mathrm{N} \quad=$ Number of expansion terms

$\mathrm{Z}_{\mathrm{I} 1}, \mathrm{Z}_{\mathrm{I} 2}=$ Termination impedance at load \#1 (west-end) and load \#2 (east-end) respectively, set equal to $10.8 \mathrm{kQ}$, essentially open circuit, the value of the voltage divider used in the experiment.

While most of the above parameter definitions are straightforward, two of them require further explanation. The horizontal polarization parameter, $\alpha_{h}$, determines what fraction of the incident electric field $E^{i}$ is horizontally polarized and what fraction of it is vertically polarized according to the following relationships:

$$
E_{v}^{i}=\alpha_{v} E^{i}
$$

and

$$
E_{h}^{i}=\alpha_{h} E^{i}
$$

such that,

$$
\alpha_{h}^{2}+\alpha_{v}^{2}=1
$$

where, $\alpha_{h}$ and $\alpha_{v}$ are the horizontal and vertical polarization coefficients, respectively, and $E_{h}^{i}$ and $E^{i}$ are the horizontal(perpendicular) and vertical- (parallel) polarized components of the incident electric field.

Thus, according to Figure $4.2, \alpha_{h}$ and $\alpha_{v}$ can, respectively, be written as $\cos \alpha$ and $\sin \alpha$ where $\alpha$ is the polarization angle. If the incident electromagnetic wave is vertically polarized, $\alpha_{h}$ is zero and this corresponds to a polarization angle, $\alpha$, of $90^{\circ}$. If the incident electromagnetic wave is horizontally polarized, $\alpha_{h}$ is one and this corresponds to a polarization angle, $\alpha$, of zero degrees. 
The second parameter that needs discussion is the number of expansion terms, $N$. The number of expansion terms is an integer ranging from 0 to 10 that corresponds roughly to the number of desired oscillations on the line that will be calculated in the transient response. If a negative integer is used, the complete response will be obtained. This time truncation is used to overt difficulties with FFT ailing for the case where the line response persists for times longer than the time duration of the input.

The EMPLIN program uses the incident electric field as an input. On the other hand, it is the sum of incident and reflected fields at or near ground that are measured in the lightning experiment. In order to determine the incident electric field, $E^{i}$, the input to EMPIIN, we have derived, employing the results obtained in Chapter IV, an analytical expression that relates $E^{i}$ to the measured north-south component of the magnetic flux density $B_{\mathrm{EW}}$. That expression is given in Eq. (8.8) .

$$
\begin{aligned}
& E^{1}(t) \sim A \sin \Phi \sum_{i=1}^{n-1}\left[-\frac{B_{1+1}-B_{i}}{t_{1+1}-t_{i}}+\frac{B_{i+2}-B_{i+1}}{t_{i+2}-t_{i+1}}\right] \cdot\left(t-t_{1+1}\right) u\left(t-t_{i+1}\right) \\
& +A \sin \Phi \frac{B_{2}}{t_{2}} \cdot t u(t) \\
& +A \frac{B_{2}}{t_{2} e_{r}^{y_{2}}} \cdot t e^{-p t}\left[I_{0}(p t)+I_{1}(p t)\right] u(t) \\
& +A \cdot \sum_{i=1}^{n-1} \frac{1}{\varepsilon_{x}^{3 / 2}}\left[-\frac{B_{i+1}-B_{1}}{t_{i+1}-t_{i}}+\frac{B_{i+2}-B_{i+1}}{t_{i+2}-t_{i+1}}\right] \cdot\left(t-t_{i+1}\right) \exp \left[-p\left(t-t_{i+1}\right)\right] \\
& \cdot\left[I_{0}\left(p\left(t-t_{1+1}\right)\right)+I_{1}\left(p\left(t-t_{1+1}\right)\right)\right] u\left(t-t_{1+1}\right)
\end{aligned}
$$


where,

$$
A=V_{c} /(\sin \alpha \cdot \sin \beta \cdot \sin 2 \Phi)
$$

and

$$
p=\frac{\sigma}{2 e_{0} e_{x}}
$$

$I_{0}$ and $I_{1}$ are the modified Bessel functions of zero and first order, respectively. The angle $\beta$ which is the angle between the two horizontal magnetic field components is approximated as follows:

$$
\tan \beta \sim \frac{\sin \Phi \sin \phi \cos \alpha+\sin \alpha \cos \phi}{\sin \Phi \cos \phi \cos \alpha+\sin \alpha \sin \phi}
$$

Eq. (8.8) is an expression for the incident electric field in terms of $n$ samples of the north-south component of the magnetic flux density for the interval $t=0$ to $t=t_{n}$. Three basic assumptions were made to obtain the analytical expression given in Eq. (8.8). (1) The measured magnetic flux density was analytically expressed using the piecewise linear approximation representation of time domain signals (e.g., Master, 1982 ). (2) For the frequencies and ground parameters involved, a convenient approximation was:

$$
\frac{1}{\left(\varepsilon_{Y}+\sigma / j \omega \varepsilon_{0}\right)^{2}}<\frac{1}{\left(\varepsilon_{I}+\sigma / j \omega \varepsilon_{0}\right)}
$$

(3) The magnitude of the reflection coefficients for both parallel- and perpendicular-polarized magnetic flux densities were assumed unity and the phase was assumed zero degrees for the frequencies involved, without introducing much error.

\subsubsection{An Overview of Coupling}

The components of the electric field which induce currents on the power line are the vertical, when the line is terminated with other than 
an open circuit, and the horizontal components that are along the line. Whereas grazing-incidence electric fields from distant ground lightning exhibit a horizontal component only in the direction of propagation, overhead fields may have, in general, horizontal electric field components both parallel to the direction of propagation (parallelpolarized electric field components) and perpendicular to the direction of propagation (perpendicular-polarized electric field components). The electric radiation field associated with the cloud discharge process is assumed to be linearly polarized in the plane $P$, perpendicular to the direction of propagation. As illustrated in Figure 8.1, the plane, P, can be divided into four distinct quadrants: $01,02,03$, and 04 . The incident electric field can be polarized in any of these four quadrants depending on the polarity of charge transported along the discharge channel and its orientation in space.

The horizontal electric field component along the power line can be divided into two "parts," one "part" being due to the parallel-polarized electric field and the other being due to the perpendicular-polarized electric field. These two "parts" can interfere constructively or destructively depending on the polarization of the incident electric field. For example, when the incident electric field is polarized in Q1, both the parallel- and perpendicular-polarized electric fields give an along-the-line component in the same direction, but when the incident electric field is polarized in $Q 2$, the parallel- and perpendicularpolarized electric fields give an along-the-line component in opposite directions.

Therefore, given the azimuth and elevation angles, the electric field polarization, determines the amplitude and direction of the alongthe-line horizontal electric field components. 


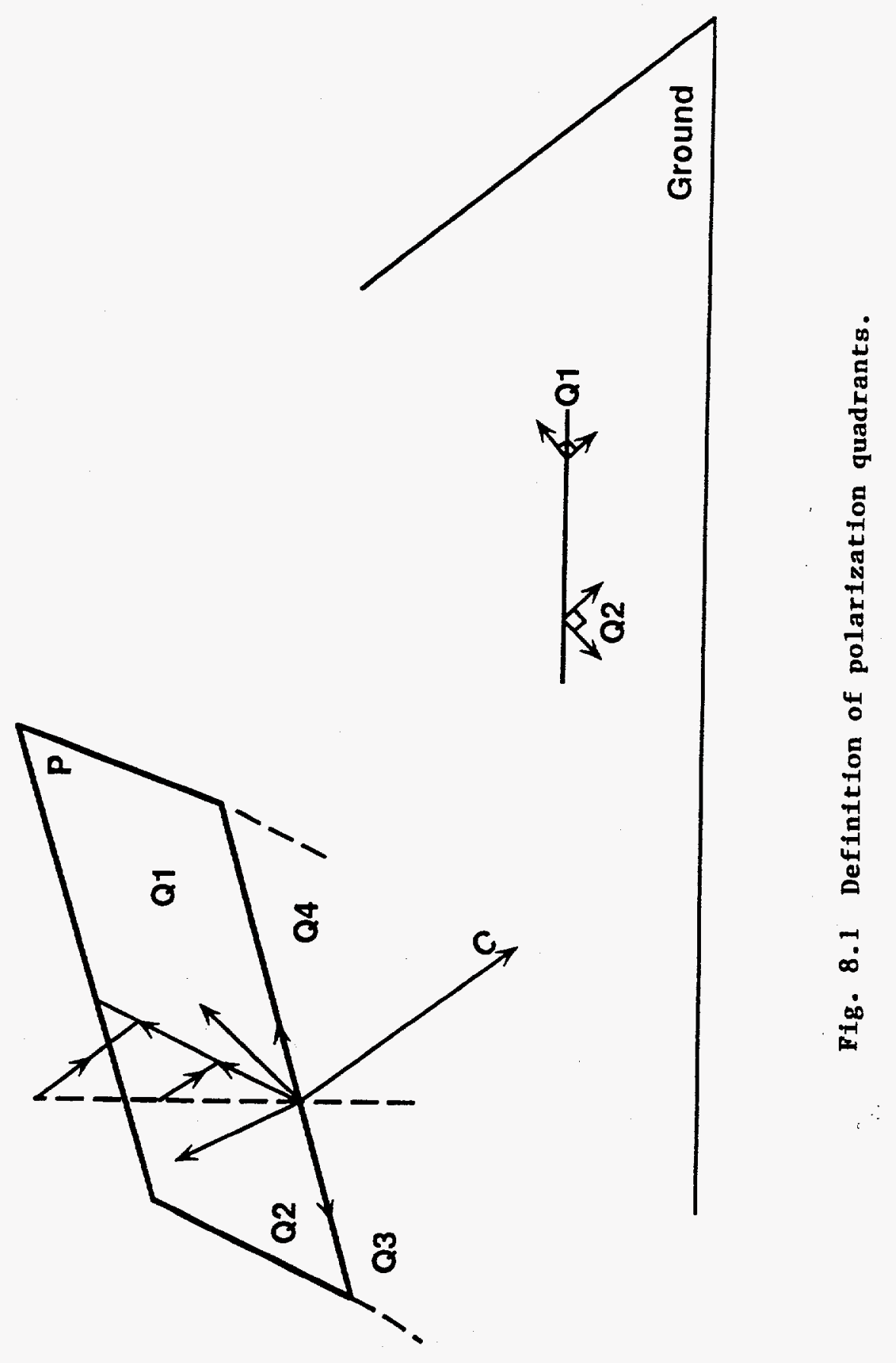


Thus far we have only examined the effect of the polarization angle on the along-the-line component of the horizontal electric field. The azimuth angle, however, can also cause the destructive/constructive interference characteristic to reverse. For example, $Q 1$ has a destructive addition characteristic for $90^{\circ}<\phi<180^{\circ}$ while it has a constructive addition characteristic for $0^{\circ}<\phi<90^{\circ}$. Table 1 gives the addition characteristics of each polarization quadrant for different azimuth regions. The NW region is defined by $0^{\circ}<\phi<90^{\circ}$, the NE region by $90^{\circ}<\phi<180^{\circ}$, the SE region by $180^{\circ}<\phi<270^{\circ}$, and the Sw region by $270^{\circ}<\phi<360^{\circ}$. In Table $1, "+^{n}$ indicates constructive interference of the two along-the-line horizontal electric field components and "-" indicates destructive interference of these components.

Tab. 8.1 -The addition characteristic of the four polarization quadrants with respect to the four regions of the azimuth plane.

\begin{tabular}{|c|c|c|c|c|}
\hline AZIMUTH & Q1 & $\begin{array}{c}\text { POLARIZATION } \\
\mathrm{Q2}\end{array}$ & $\begin{array}{c}\text { QUADRANT } \\
\text { Q3 }\end{array}$ & Q4 \\
\hline NW & + & - & + & - \\
\hline $\mathrm{NE}$ & - & + & - & + \\
\hline SE & + & - & + & - \\
\hline SW & - & + & - & + \\
\hline
\end{tabular}

Moreover, given a polarization in $Q 2$ or 04 , and given the azimuth angle, $\phi$, varying the elevation angle, $\Phi$, can change the direction of the induced current due to the horizontal electric field (Yacoub, 1990).

\subsection{Analysis}

We have generated a model of the measured voltages for each of the five overhead cloud events whose horizontal magnetic flux densities are shown in Fig. 2.6 from Chapter 2 using EMPLIN by trying different combinations of $\alpha, \phi, \Phi$, and $\sigma$ and then chosing the combination which 
gives the best fit to the measured voltages. We used different techniques to narrow down the possible ranges of the three angles $(\alpha, \phi$, and $\Phi)$ involved in the voltage calculations. The following definitions and classifications will help the reader to understand these techniques:

(1) The factor $A$ in Eq. (8.8) has no effect on the waveshape of the calculated electric field, but, it does affect its amplitude and polarity. In order to investigate the polarity of the factor $A$ as a function of the three angles, tables were generated by computing the value of $\mathrm{A}$ (by means of $\mathrm{Eq} .(8.9)$ ) with one angle $(\alpha, \phi$, or $\Phi$ ) as a variable parameter and the other two left constant. An example of such a table is given in Table 2. Other tables similar to Table 2 were generated for different values of $\alpha$ and $\Phi$. Thus, for a given polarity of the north-south component of the magnetic field, $B_{\mathrm{Ew}}$, according to Eq. (8.8), we can either obtain an incident electric field that has the same polarity as $B_{E w}$, if $A$ is positive, or an incident electric field that has an opposite polarity of $B_{E y}$, if $A$ is negative.

(2) The usual lightning convention is used. That is, a positive electric field is defined as one whose electric field lines point toward the ground which will produce a positive excitation voltage, $v^{\circ}(x)$, according to Eq. $(8.4)$.

The techniques used to narrow down the possible ranges of the three angles involved in the voltage calculations will now be discussed. We were able to use a three-station time delay technique, where the three stations are the two ends of the overhead test line and one of the magnetic flux density antennas, to determine crudely the direction to the source. This technique has been described in detail in section 2.2.2. By separately overlaying the end voltage waveforms on top of the $B_{\mathrm{gw}}$ waveform, we are able 
Tab. 8.2 -The variation in $A$ as a function of azimuth for $\alpha=80^{\circ}$ and $\Phi=80^{\circ}$.

\begin{tabular}{ccc} 
ALPHA & AZIMUTH & $\mathrm{A}$ \\
\hline 80.0 & 0.0 & -1 \\
20.0 & $0.90400173 \mathrm{E}+09$ \\
40.0 & $0.99806707 \mathrm{E}+09$ \\
60.0 & $0.11887619 \mathrm{E}+10$ \\
80.0 & $0.15798826 \mathrm{E}+10$ \\
90.0 & $0.27699866 \mathrm{E}+10$ \\
100.0 & $0.52059418 \mathrm{E}+10$ \\
120.0 & $-0.32229802 \mathrm{E}+12$ \\
140.0 & $-0.21757315 \mathrm{E}+10$ \\
160.0 & $-0.11290008 \mathrm{E}+10$ \\
180.0 & $-0.90886835 \mathrm{E}+09$ \\
200.0 & $-0.90400173 \mathrm{E}+09$ \\
220.0 & $-0.99806707 \mathrm{E}+09$ \\
240.0 & $-0.11887619 \mathrm{E}+10$ \\
260.0 & $-0.1579883 \mathrm{E}+10$ \\
270.0 & $-0.27699860 \mathrm{E}+10$ \\
280.0 & $-0.52059428 \mathrm{E}+10$ \\
300.0 & $0.32225277 \mathrm{E}+12$ \\
320.0 & $0.21757297 \mathrm{E}+10$ \\
340.0 & $0.11290008 \mathrm{E}+10$ \\
& $0.90886842 \mathrm{E}+09$
\end{tabular}

Tab. 8.3 -The convention used by EMPLIN to determine in which quadrant the incident electric field lies.

\begin{tabular}{|c|c|c|}
\hline Polarity of $E^{i}$ & Sign of $\alpha_{h}$ & Quadrant \\
\hline positive & positive & $\mathbf{Q 1}$ \\
\hline negative & positive & $\mathbf{Q 3}$ \\
\hline positive & negative & $\mathbf{Q}$ \\
\hline negative & negative & $\mathbf{Q 4}$ \\
\hline
\end{tabular}

to determine with a reasonable degree of confidence whether the electromagnetic wave arrived at the distribution line from an easterly or a westerly direction. This technique is more effective for elevation angles lower than $50^{\circ}$ since, for these angles, the delay time between either end of the distribution line and the magnetic flux density antenna is observed more readily. For elevation angles higher than $50^{\circ}$, the easterly or westerly direction of the incident electromagnetic wave can 
be determined by comparing the widths of the initial pulses in the measured east- and west- end voltages of the cloud event, and comparing them to the ideal voltage response study of the line (Yacoub, 1990). When both ends of the distribution line are open circuited, wider initial measured voltage pulses suggest that the incident electric field struck the end which has the wider initial voltage pulse first. This is because at the end which is struck first, the initial voltage pulse is mostly due to the vertical component of the electric field whereas, at the other end, the initial voltage pulse is due to both the vertical and horizontal components of the electric field. The elevation angle was determined to be higher or lower than $50^{\circ}$ as follows. Simulations show that the response of the distribution line is typical of an overhead excitation (similar waveshape opposite polarity voltages at either end), for elevation angles higher than $50^{\circ}$. When these typical waveshapes were not observed, elevation angles lower than $50^{\circ}$ were investigated. For a given polarity of the measured $B_{E w}$ waveform, and after determining whether the electomagnetic wave is incident at the line from an easterly or a westerly direction, the polarization of the incident electric field can be determined from the initial pulse polarity of the measured end voltage. The specific quadrant can be determined by using the destructive/constructive interference property of the azimuth plane relative to the polarization quadrant summarized in Table 1 . These techniques proved very effective in modeling the measured voltages. 8.3 Results

In Figure 8.2 we show the calculated incident electric field for the five events analyzed derived from the measured north-south component of the magnetic field using Eq. (8.8). 
Event1

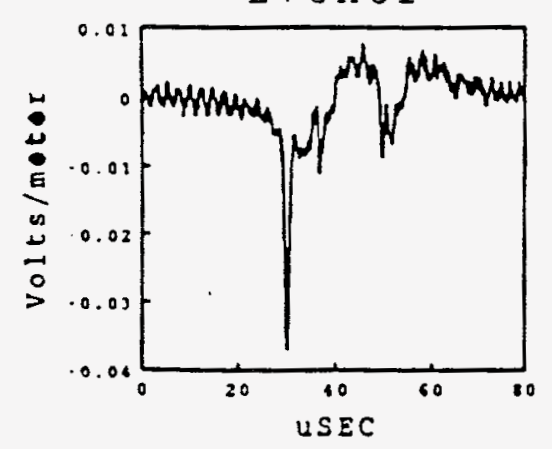

Bvent 19
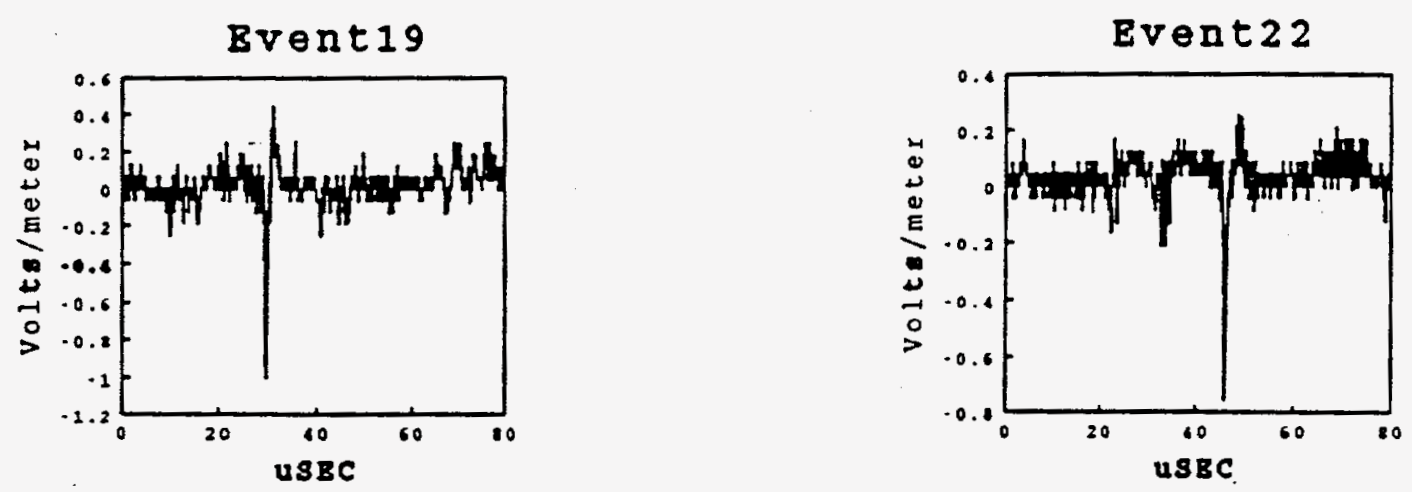

Event 31
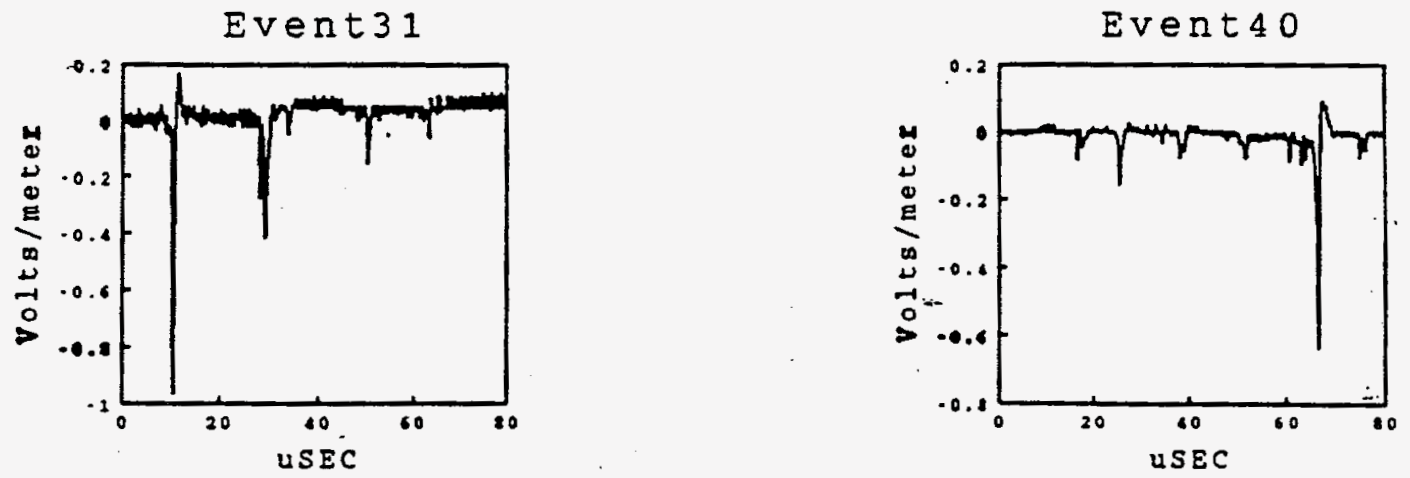

Fig. 8.2 Calculated incident electric field for five events. 
As is evident from comparing Figure 4.3 and Figure 2.6, the calculated incident electric fields are very similar in waveshape to the measured magnetic fields.

For each example, the combination of the direction angles was first reduced to the smallest region possible using the factors mentioned earlier. The next step was to try different combinations of $\alpha, \Phi$, and $\phi$ in that region searching for the best fit to the measured voltage waveshapes. The conductivity of the ground was then varied within a certain range to change subtle waveform characteristics like pulse widths, relative pulse amplitudes, overall waveform amplitudes, and sudden and narrow amplitude variations which we will call "glitches".

We illustrate the analysis technique with one of the above mentioned five events, event 19 of flash 86. By separately overlaying the end voltage waveforms on top of the $B_{E w}$ waveform and comparing the initial pulse widths ( the initial pulse width of the east voltage is wider than the initial pulse width of the west voltage) of the two end voltages, one can infer that the electric field was incident to the line from an easterly direction. Also, since the end voltage waveforms looked very much like those due to an overhead excitation and because there was virtually no time delay between the two end voltage waveforms, one can conclude that the elevation angle was higher than $50^{\circ}$. Elevation angles in the range of $50^{\circ}$ to $90^{\circ}$ were tried. Since $B_{E W}$ for this event is a negative polarity bipolar pulse incident to the line from an easterly direction and the initial peak of the east voltage has a positive polarity, the polarization of the incident field has to be in either quadrant three or four and the constant $A$ has to be negative in order to obtain the correct initial pulse polarity of the calculated east voltage. Moreover, the interaction of the parallel and perpendicular horizontal 
electric field components has to be constructive, otherwise no ringing would be apparent on the line because the resultant horizontal electric field component along the line would be negligible. Thus, according to Table 1, for constructive interference in $Q 3$ the correct azimuth region is the SE region, and for constructive interference in $Q 4$ the correct azimuth region is the NE region.

After a number of trials using direction angles within the narrowed regions above, the combination that produced the best match to the measured voltages was: $\alpha=350^{\circ}, \phi=140^{\circ}$, and $\Phi=80^{\circ}$. Conductivities of $1.6 \times 10^{-2}, 1.0 \times 10^{-2}, 0.5 \times 10^{-2}$, and $0.25 \times 10^{-2}$ mho/m were used with the above combination. The results are shown in Figure 8.3 . The bottom pair of Figure 8.3 with conductivity equal to $0.25 \times 10^{-2}$ gives a better match in the difference between the first and second peaks of the same polarity. Varying the elevation angle will not affect the overall waveshapes of the two end voltages $(e . g .$, changing the polarities of the initial pulses of the two end voltages) because the incident electric field is almost completely perpendicularly polarized $\left(\alpha=350^{\circ}\right)$ and thus the effect of the vertical electric field is negligible. However, other fine structure on the two end voltages will change as the elevation angle is varied. For example, decreasing the elevation angle to $50^{\circ}$ for this example, causes the transition between consecutive reflections not be as smooth as it was at the elevation angle of $80^{\circ}$. This is illustrated in Figure 8.4 .

The same procedure that was followed in the previous example to obtain the best match was also used for the remaining four example events. 

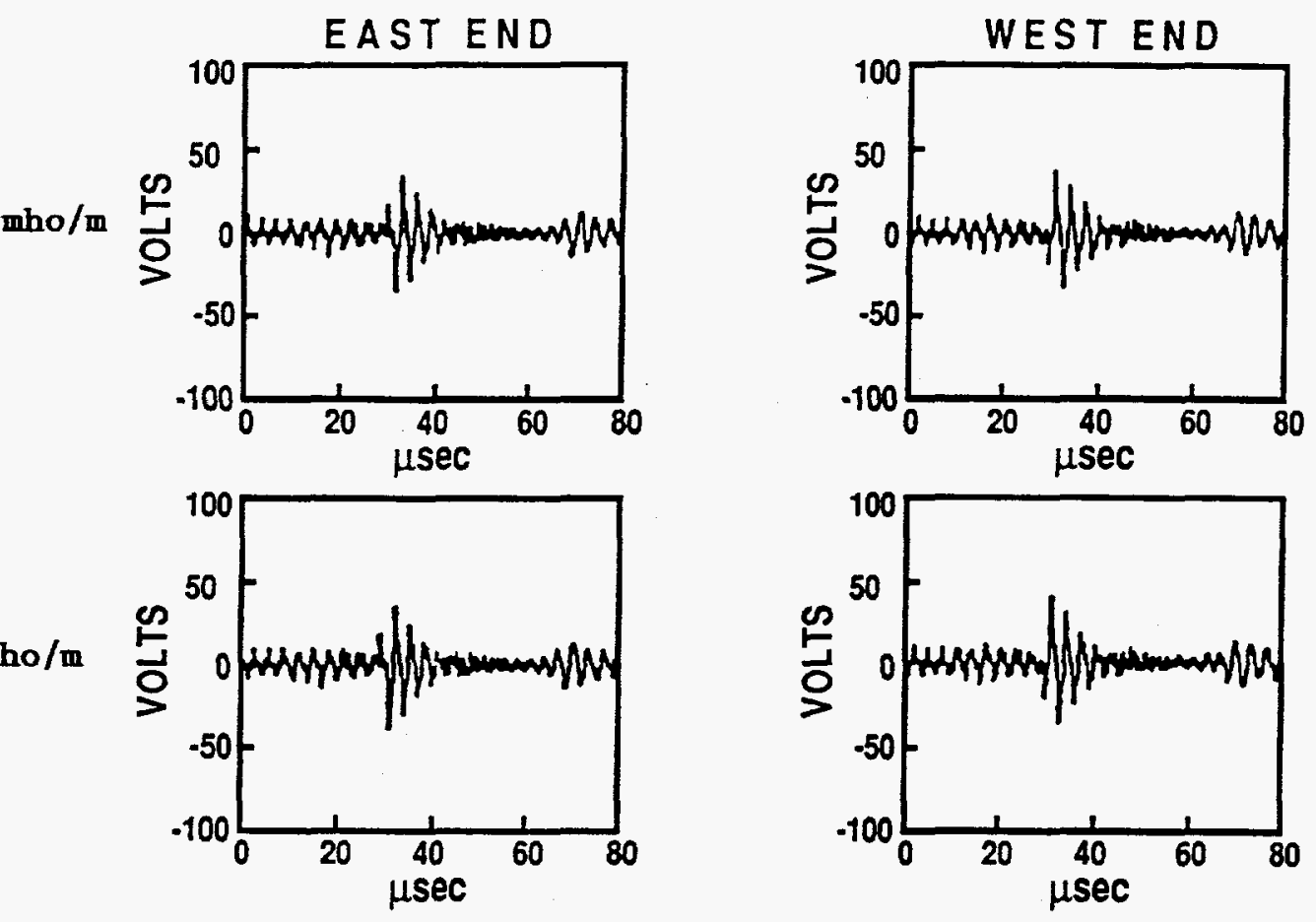

$=1.0 \times 10^{-2} \mathrm{mho} / \mathrm{m}$
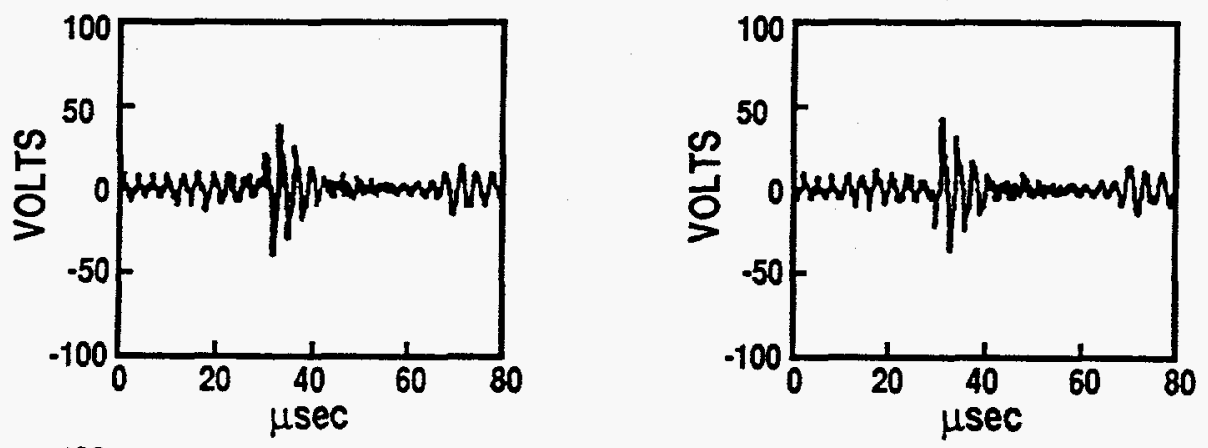

$=0.5 \times 10^{-2} \mathrm{mho} / \mathrm{m}$
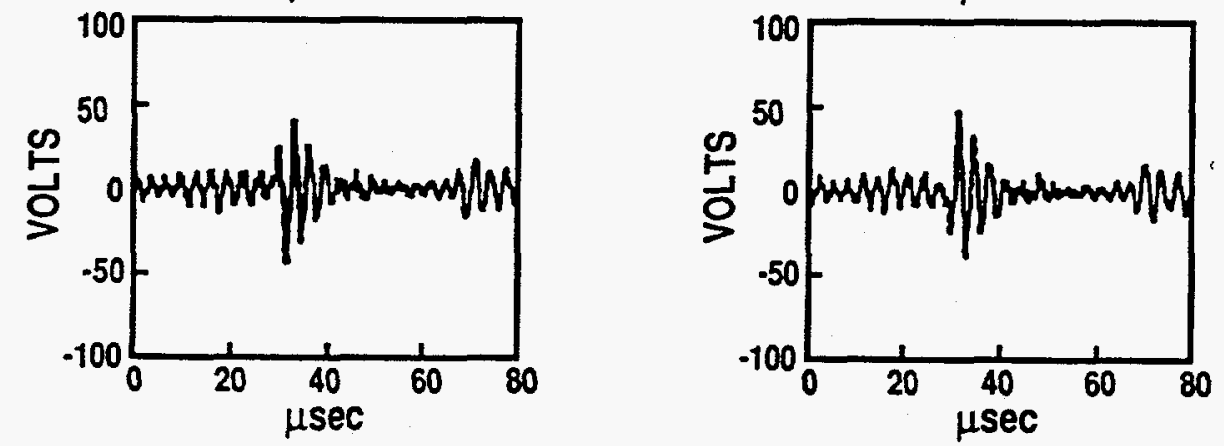

Fig. 8.3 Effect of the ground conductivity on the simulated voltages for event 19 of flash 86. From top to bottom, the pairs of voltages were calculated with $0=1.6 \times 10^{-2}, 1.0 \times 10^{-2}, 0.5$ $\times 10^{-2}$, and $0.25 \times 10^{-2} \mathrm{mho} / \mathrm{m}$. 

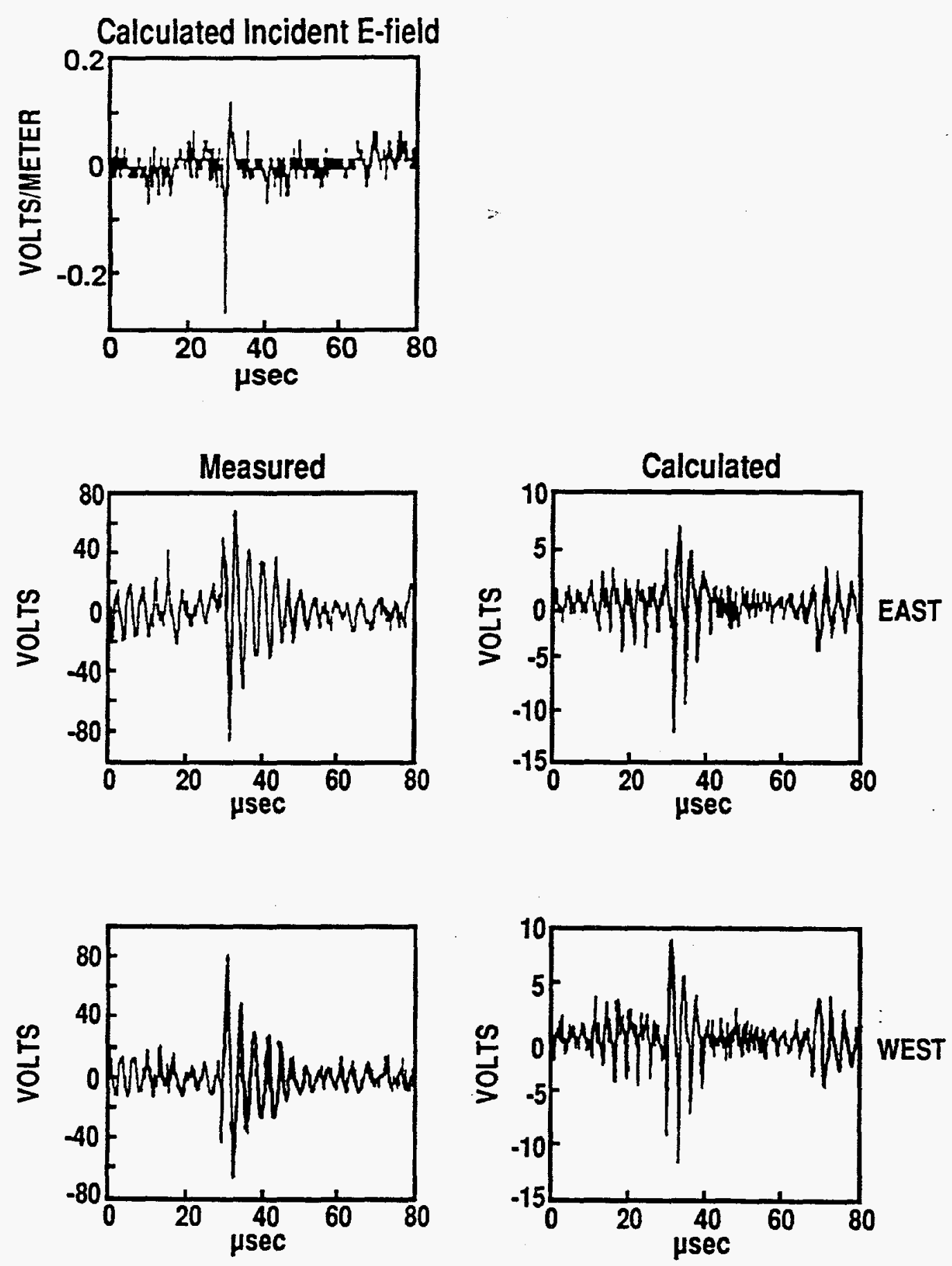

Fig. 8.4 Illustration of the effect of lowering the elevation angle in the simulation of event 19 of flash 86 
The combinations that gave the best similarity to the measured voltages were: $\alpha=260^{\circ}, \phi=70^{\circ}$, and $\Phi=20^{\circ}$ for event 1, flash $52 ; \alpha=355^{\circ}, \phi$ $=320^{\circ}$, and $\Phi=80^{\circ}$ for event 31 , flash $60 ; \alpha=350^{\circ}, \phi=300^{\circ}$, and $\Phi=$ $80^{\circ}$ for event 40 , flash $60 ;$ and $\alpha=350^{\circ}, \phi=90^{\circ}$, and $\Phi=80^{\circ}$ for event 22 , flash 86 . Conductivities of $1.6 \times 10^{-2}, 1.0 \times 10^{-2}, 0.5 \times 10^{-2}$, and $0.25 \times 10^{-2}$ were used with the above combinations. A conductivity of $0.25 \times 10^{-2}$ gave the best match in every case. All results are similar to those shown in Figure 8.3. In the five examples, if the calculated incident electric field were doubled, the comparison between measured and calculated voltage amplitudes would be more favourable.

\subsection{Discussion}

We have illustrated the ability of EMPLIN to predict the voltage response at the two ends of a power line from overhead sources of electromagnetic radiation. Even though neither the direction angles of the lightning fields nor the effective conductivity of the ground were accurately known, the calculated voltage waveforms are in fairly good agreement with the measured ones for reasonable values of these parameters. However, it is important to note that the combination of the direction angles and the conductivity chosen as those which gives the best fit between theory and experiment are not unique. For example, in event one of flash 52 , if we increase the elevation angle to $30^{\circ}$, a conductivity of $0.5 \times 10^{-2} \mathrm{mho} / \mathrm{m}$ could be used to obtain almost the same degree of agreement between theory and experiment as for an elevation angle of $20^{\circ}$ and a conductivity of $0.25 \times 10^{-2} \mathrm{mho} / \mathrm{m}$.

A significant point of interest is that the effective ground conductivity that provides the best fit between theory and measurement is smaller than the value of $1.6 \times 10^{-2} \mathrm{mho} / \mathrm{m}$ determined from measurements of horizontal and vertical components of the electric field (e.g., Tzeng, 
1986) and used as the effective ground conductivity to model the induced voltages on the same power line from fields incident along the earth from cloud-to-ground lightning return strokes (e.g., Rubinstein et al., 1989; Georgiadis et al., 1992) and for nearby artificially initiated lightning in Chapter VII. The present value is about three $\left(0.5 \times 10^{-2}\right)$ to six $\left(0.25 \times 10^{-2}\right)$ times smaller. While the ground conductivity is not homogeneous, we necessarily use one effective ground conductivity in the time- and in the frequency-domain coupling programs. The result of assuming an effective ground conductivity, instead of a multiple layer ground, on the amplitude and waveshape of the horizontal electric field at ground level for grazing-incidence electromagnetic waves was studied by Thomson et al. (1988b) for a site that is different from that at which this and the previous studies (e.g., Georgiadis et al., 1992) were performed. Thomson et al. $(1988 \mathrm{~b})$ were able to use the Wenner four-probe method to model the ground of their experiment site as a four layer ground from the surface to a depth of 11 meters. They showed that using the conductivity of the top layer as the effective conductivity of the ground caused the peaks of the calculated horizontal electric field to be about 33 per cent wider and larger than the measured field peaks. According to Thomson et al. $(1988 \mathrm{~b})$ the calculated horizontal electric field peaks were overestimates of the true field peaks because for frequencies typical of the peak grazing-incidence horizontal field, the field penetrates (skin depth effect) enough into the second layer, with a higher conductivity, so that a higher effective conductivity will probably give a better comparison between calculated and measured fields. Unfortunately, problems were encountered in utilizing the wenner four-probe method to model the ground conductivity of the site at which this study was made in the same way that the site of Thomson et al. 
(1988b) was modeled probably because the ground was not homogeneous or horizontally stratified. However, we do know that the site of this study was near a body of salt water which probably means that a highly conductive layer is present below the surface. Since the effective conductivity of $1.6 \times 10^{-2} \mathrm{mho} / \mathrm{m}$ was determined from measurements of grazing-incidence horizontal and vertical electric field components (e.g., Tzeng, 1986) which have a lower frequency content and penetrate the earth deeper than overhead signals, we suspect that the deeper (more conductive) layer influenced his measurement and, thus, resulted in a higher effective conductivity than the one determined in this study. In the overhead lightning study, the higher frequency component fields from the overhead source have a relatively small skin depth and hence are affected more by the upper, less conducting layer.

Other reasons for the difference in effective ground conductivities between the overhead study and in studies of ground level sources are the following: (1) The horizontal electric field component at line height in the overhead study is calculated from a different conductivity (and frequency) dependent expressions than the horizontal field from sources at grazing incidence. In the overhead study, the horizontal electric field component is calculated from the incident electric field via a reflection coefficient whereas in the studies previously done by the UF Lightning Research Group for grazing incidence, the horizontal electric field was calculated from the vertical field using the wavetilt formula, a method which is valid only at ground level (e.g., Master and Uman, 1984; Thomson et al., 1988b; Rubinstein et al., 1989; Georgiadis et al., 1992). The appropriate effective conductivity in each approach might be different. (2) EMPLIN uses an expression for the ground impedance which, in addition to being frequency dependent, depends on the ground 
conductivity. Therefore, unlike the previous studies where the ground impedance was assumed constant (e.g., Master and Uman, 1984; Rubinstein et al., 1989; Georgiadis et al., 1992), in the overhead study the ground conductivity not only plays a role in the determination of the horizontal electric field along the line as it does for grazing incidence, but also affects directly the calculation of the scattered voltage along the line. (3) The shielding effect of the trees is probably more pronounced in the case of fields at grazing incidence than on those which are overhead. For the grazing incidence case, the vertical electric field tends to be reduced by the trees. Thus, the vertical electric field acting on the line will be lower than what is actually measured some distance away. In other words, the wavetilt ratio for grazing incidence is actually higher than it is thought to be, and, because $W(s)=f\left(1 / \sigma^{4}\right)$, the effective conductivity of $1.6 \times 10^{-2} \mathrm{mho} / \mathrm{m}$ used in Rubinstein et al. (1989) and Georgiadis et al. (1992) may be higher than what should have been used. 


\section{REFERENCES}

Agrawal, A. K., H. J. Price, and S. H. Gurbaxani, Transient response of multiconductor transmission lines excited by a non-uniform electromagnetic field, IEEE Trans. EMC, EMC-22: 119-129 (1980).

Aigner, V., 1935. Induzierte Blitzuberspannungen und ihre Beziehung zum ruckwartigen Uberschlag. ETz 56, 497-500.

Al-Tai, M. A., H. S. B. Elayyan, D. M. German, A. Haddad, N. Harid, and R. T. Waters, The simulation of surge corona on transmission lines, IEEE Trans. PD, PD-4, 1989 .

Carson, J. R., Wave propagation in overhead wires with ground return, Bell System Technical Journal, 5:539-554 (1926).

Cianos, N., G.N. Oetzel, and E.T. Pierce, A technique for accurately locating lightning at close ranges, J. Appl. Meteor, 11, 1120-1127 (1972).

Diendorfer, G., and M. A. Uman, An improved return stroke model with specified channel-base current, J. of Geophys. Res., 95:13,621-13,644 $(1990)$.

Gary, C., Approche complete de la propagation multifilaire en haute frequence par utilisation des matrices complexes, E.D.F. bulletin de la Directiondes Etudes et Recherches, Serie B, 5-10 (1976).

Georgiadis, N., Voltages at both ends of a test power line induced by lightning at the Kennedy space Center in 1986, Master's Thesis, University of Florida (1988).

Georgiadis, N., M. Rubinstein, M. A. Uman, P. J. Medelius, and E. M. Thomson, Lightning-induced voltages at both ends of a $450-\mathrm{m}$ distribution line, IEEE Trans. EMC, 34, 451-460 (1992).

Ianoz, M., C.A. Nucci, and F.M. Tesche, Transmission line theory for field-to-Transmission line coupling calculations, Electromagnetics, 8 , 171-211 (1988).

Idone, V.P., R. E. Orville, P. Hubert, L. Barret, and A. Eybert-Berard, Correlated observations of three triggered lightning flashes, J. Geophys. Res., $89: 1,385-1,394$ (1984).

Kitagawa, N., and M. Brook, A comparison of intracloud and cloud-toground lightning discharges, J. Geophys. Res., 65, 1189-1201 (1960).

Koga, H., T. Motomitsu, and M. Taguchi, Lightning surge waves induced in transmission lines, Review of the Electrical Communication Laboratory, 29:797-810 (1981).

Krider, E.P., G.J. Radda, and R.C. Noggle, Regular radiation field pulses produced by intracloud lightning discharges, J. Geophys. Res., 80, 38013804 (1975). 
Leteinturier, C., J. Hamelin, L. Nicot, C. Weidman, A. Eybert-Berard, L. Barret, and J.P. Berlandis, Lightning current and current-derivative measurements, proceedings of the $8 \mathrm{th}$ International Conference on Atmospheric Elctricity, Uppsala, Sweden (1988a).

Leteinturier, C., J. Hamelin, L. Nicot, C. Weidman, J. Willett, and E. P. Krider, Lightning current-derivative and electric field-derivative correlation-measurements and models, Proceedings of the 8 th International Conference on Atmospheric Elctricity, Uppsala, Sweden (1988b).

Leteinturier, C., C. Weidman, J. Hamelin, Current and electric field derivatives in triggered lightning return strokes, J. of Geophys. Res, $95: 811-828$ (1990).

LeVine, Sources of the strongest RF radiation from lightning, J. Geophys. Res., 85, 4091-4095 (1980).

Lewis, E.A., R.B. Harvey, and J.E. Rasmussen, Hyperbolic direction finding with sferics of transatlantic origin, J. Geophys. Res., 65, 18791905 (1960).

Lin, Y.T., M.A, Uman, J.A. Tiller, R.D. Brantley, W.H. Beasley, E.P. Krider, and C.D. Weidman, Characterization of lightning return stroke electric and magnetic fields from simultaneous two-station measurements, J. Geophys. Res., 84, 6307-6314 (1979).

Lundholm, R., Induced overvoltage surges on transmission lines and their bearing on the lightning performance on medium voltage networks, Trans. Chalmers Univ. of Tech., No. 188, Gothenburg, Sweden (1957).

MacClement, W.D., and R.C. Murty, VHF direction finder studies of lightning, J. Appl. Meteor:, 17, 786-795 (1978).

Master, M. J., Lightning induced voltages on power lines: theory and experiment, Ph.D. dissertation, University of Florida, Gainesville, Florida (1982).

Master, M. J., and M. A. Uman, Lightning induced voltages on power lines: Theory, IEEE Trans. PAS, PAS-103:2505-2517 (1984).

Master, M. J., M. A. Uman, W. Beasley, and M. Darveniza, Lightning induced voltages on power lines: Experiment, IEEE PAS Trans., PAS-103:2519-2529 (1984).

Murty, R.C., and W.D. MacClement, VHF direction finder for lightning location, J.Appl. Meteor., 12, 1401-1405 (1973).

Norton, K. A., Propagation of radio waves over a plane earth, Nature, $135: 954-955(1935)$.

Norton, K. A., Propagation of radio waves over the surface of the earth and in the upper atmosphere-Pt II, Proc. IRE, 25:1203-1236 (1937).

Proctor, D.E., A hyperbolic system for obtaining VHF radio pictures of lightning, J. Geophys. Res., 76, 1478-1489 (1971). 
Proctor, D.E., A radio study of lightning, Ph.D. thesis, University of Witwatersrand, Johannesburg, S.A. (1976).

Proctor, D.E., VHF radio pictures of cloud flashes, J. Geophys. Res., 86, 4041-4071 (1981a).

Rachidi, F., and C. A. Nucci, on the Master, Uman, Lin, Standler and the modified transmission line lightning return stroke current models, submitted for publication (1990).

Rubinstein, M., A theoretical study of the relation between vertical and horizontal electric fields at ground level from lightning, Master's Thesis, University of Florida (1986).

Rubinstein, M., A. Y. Tzeng, M. A. Uman, P. J. Medelius, and E. M. Thomson, An experimental test of a theory of lightning-induced voltages on an overhead wire, IEEE Trans. EMC, EMC-31:376-383 (1989).

Rusk, S., Induced lightning over-voltages on power-transmission lines with special reference to the over-voltage protection of low-voltage networks, Trans. Roy. Inst. of Tech., No. 120, Stockholm, Sweden $(1958)$.

Sommerfeld, A., Uber die ausbreitung der wellen in der drahtlosen telegraphie, Annalen der Physics (4), 28:665 (1909).

Stratton, J.A., Electromagnetic theory, McGraw-Hill, New York (1941).

Sunde, E. D., Earth conduction effects in transmission systems, Dover, New York (1968).

Szpor, S., A new theory of induced overvoltages, CIGRE, Report \#308, Paris (1948).

Taylor, W.L., A VHF technique for space-time mapping of lightning discharge processes, J. Geophys. Res., 83, 3575-3583 (1978).

Taylor, C. D., R. S. Satterwhite, and C. W. Harrison, The response of a terminated two-wire transmission line excited by a non-uniform electromagnetic field, IEEE Trans. AP, AP-13:987-989 (1965).

Thomson, E. M., P. J. Medelius, and M. A. Uman, A remote sensor for the three components of transient electric fields, IEEE Trans. IE, IE-35:426433 (1988a).

Thomson, E. M., P. J. Medelius, M. Rubinstein, M. A. Uman, J. Johnson, and $J$. W. Stone, Horizontal electric fields from lightning return strokes, J. of Geophys. Res., 93:2429-2441 (1988b).

Tzeng, A. Y., Coupling of lightning electromagnetic fields to power lines, Master's Thesis, University of Florida, Gainesville, Florida $(1986)$.

Vance, E. F., Coupling to Shielded Cables, John Wiley and Sons, Inc., New York $(1978,1987)$. 
Wait, J.R., Electromagnetic wave theory, Harper and Row Publishers, New York (1985).

Weidman, C.D., and E.P. Krider, The radiation field wave forms produced by intracloud lightning discharge processes, J. Geophys. Res ., 84, 3159 3164 (1979).

Weyl, H., Ausbreitung elektromagnetischer wellen ïber einer ebenen leiter, Ann. Physik, 60:481-500 (1919).

Yacoub, Z., Power line induced voltages from overhead lightning, University of Florida, Gainesville, Master's thesis (1990).

Zenneck, J., Wireless Telegraphy, Trans. from German by A. E. Seeling, McGraw Hill, New York (1915). 


\section{INTERNAL DISTRIBUTION}

$\begin{array}{ll}1 . & \text { P. R. Barnes } \\ 2 . & \text { G. E. Courville } \\ 3 . & \text { W. P. Dykas } \\ 4 . & \text { Nancy Jett } \\ 5 . & \text { Brendan Kirby } \\ 6 . & \text { D. A. Lombardi } \\ 7 . & \text { B. W. McConnell } \\ 8 . & \text { Naomi Myers } \\ 9 . & \text { C. G. Rizy } \\ 10-19 . & \text { D. T. Rizy }\end{array}$

20. R. B. Shelton

21. John Stovall

22. Jim VanCoevering

23. Phil Wolfe

24. ORNL Patent Office

25. Central Research Library

26. Document Reference Section

27. Y-12 Technical Library

28-30. Laboratory Records

31. Laboratory Records-RC

\section{External Distribution}

32. J. F. Buch, Chairman of IEEE Distribution Design Working Group, Wisconsin Electric Power Company, 231 West Michigan Street, P.O. Box 2046 P279, Milwaukee, WI 53201

33. Dr. Prit Chowdhuri, Tennessee Technological University, Box 5032, Cookeville, Tennessee 38505

34. W.E. Feero, Electric Research \& Management Inc., P.O. Box 165, State College, PA 16804

35. A.T. Giuliante, IEEE Technical Council, GEC Alsthom T\&D Inc., 4 Skyline Drive, Protection \& Control Div., Hawthrone, NY 10532

36. Imre Gyuk, Department of Energy, Office of Energy Management EE-141, 1000 Independence Ave. SW, Washington, DC 20585

37. W. Jafferis, NASA, Mail Code TP, Kennedy Space Center, FL 32899

38. E.P. Krider, Institute of Atmospheric Physics, University of Arizona, Tucson, AZ 85721

39. L.J. Lanzerotti, ATT\&T Bell Labs, Rm le-439, Murrary Hill, NJ 07974

40. J. Vito Longo, Electric Power Research Institute, Box 10412, Palo Alto, CA 94303 
41. Phil Overholt, Department of Energy, Office of Energy Management EE-141, 1000 Independence Ave. SW, Washington, DC 20585

42. V.H. Tahiliani, Electric Power Research Institute, Box 10412, Palo Alto, CA 94303

43. F.M. Tesche, 6714 Norway Rd, Dallas, TX 75230

44. M.A. Uman, Department of Electrical Engineering, Univeristy of Florida, Gainsville, FL

45. Office of Assistant Manager for Energy, Research, and Development, DOE-ORO, P.O. Box 2001, Oak Ridge, TN 37831.

46-48. OSTI, U.S. Department of Energy, P.O. Box 62, Oak Ridge, TN 37831. 